\section{Early Entrance}

Accelerated Learning

\section{Early Maladaptive Schemas}

Schemas are cognitive knowledge structures that serve to consistently bias our interpretations of events. Some of these schemas are derived early in the developmental process, and some of these early schemas provide maladaptive interpretations of events. In other words, some of these schemas typically lead to distorted preconceptions, invalid assumptions, and unrealistic goals and expectations.

\section{Early Maladaptive Schemas: The Moderating Effects of Optimism}

\author{
John R. BurI ${ }^{1}$, Amy GunTY ${ }^{2}$
}

${ }^{1}$ Department of Psychology, University of St. Thomas, Saint Paul, MN, USA

${ }^{2}$ Department of Psychology, University of Minnesota, Minneapolis, MN, USA

\section{Synonyms}

Cognitions; Early schemas; Schema-based learning

\section{Definition}

Schemas are cognitive knowledge structures that affect the ways in which we select, interpret, organize, and evaluate life experiences. Some of these schemas are developed at a young age. It is these "early schemas" that tend to be especially pervasive, rigid, stable, and enduring. Obviously, not all of these early schemas are healthy and constructive; some of them are maladaptive. Young (1999) has asserted that these Early Maladaptive Schemas fall into five clusters: (a) a sense of interpersonal disconnection and rejection, (b) a concern for one's personal autonomy, (c) an impaired ability to stay within realistic personal limits, (d) an inability to consistently express one's personal needs, wants, and desires, and (e) an excessive concern about mistakes, resulting in overvigilance to potential mistakes and inhibitions to spontaneous behaviors. Each of these Early Maladaptive Schema clusters play crucial roles in the selection of incoming psychological information, the interpretation of that information, the guiding of future behavior deriving from that information, and the storage of the resulting psychological knowledge in memory.

\section{Theoretical Background}

For over a century, psychologists have argued that numerous parental behaviors have wide-ranging and significant influence on the thoughts, behaviors, and emotions of children (Maccoby 2007). While the theories explaining this relationship have shifted historically with the psychological paradigm of the time, the conviction has remained that parenting behaviors influence many aspects of children's internal and external lives.

Within this context, Piaget (1954) and Bowlby (1980) were instrumental in the seminal suggestions that internal working models of reality are actively generated by children (even at a relatively young age), and that parental practices often contribute in significant ways to the development of these schemas. These theorists went on to assert that the resulting schemas then serve as an organizational framework for the ways in which children make sense of their myriad experiences (sometimes well into their adult years).

One parenting practice that has a particularly strong link to maladaptive outcomes for children and 
adolescents is authoritarian parenting, in which parents attempt to exercise rigid control over their children with little use of reasoning and rare occasions of parent-child rational give-and-take. Authoritarian parenting is predictive of numerous maladaptive developmental characteristics, for example, lower scholastic achievement, poorer sense of social responsibility, lower levels of self-esteem, lessened spontaneity, poorer problem-solving skills, greater discontent, and more distrust of others. Furthermore, recent evidence has linked parental authoritarianism to the presence of Young's (1999) Early Maladaptive Schemas (Buri and Gunty 2008).

Despite the abundance of evidence linking parental authoritarianism with a whole host of developmental deficiencies, not all individuals who experience unhealthy authoritarian parenting within their family of origin follow an unhealthy path of development. For some individuals, despite their authoritarian upbringing, they emerge from their childhood with a comparatively healthy developmental trajectory. It seems, therefore, that some individuals have a resilience factor (Walsh 2006) that serves as a buffer between family background and maladaptive psychological development.

\section{Important Scientific Research and Open Questions}

Optimism is the perception that there is more positive than negative in life and that things (at least in the long run) will work out for the best. As Seligman (2002) put it, for the optimistic individual, when unpleasant events occur, they are seen as temporary and limited. For the pessimistic individual, however, those same unpleasant events are seen as permanent and pervasive.

Optimism was investigated as a resilience factor, a buffer moderating the impact of parental authoritarianism on the development of Early Maladaptive Schemas. It was found that those individuals who had the lowest levels of optimism and who had the most authoritarian parents revealed the strongest levels of Early Maladaptive Schemas. On the other hand, for those individuals who had the highest levels of optimism, even though they were among those with the most authoritarian parents, there was a minimal cognitive presence of the Early Maladaptive Schemas. In fact, for this latter group, the presence of the Early
Maladaptive Schemas was equivalent to that for those individuals who were not exposed to authoritarian parenting. In other words, it was found that the deleterious effects of parental authoritarianism upon the development of Early Maladaptive Schemas were strongly moderated by optimism.

Optimism provides individuals with a positive outlook, an outlook that serves to moderate unpleasant circumstances and to enhance pleasant ones. Optimism serves to build the internal personal resources (i.e., resilience) of the individual. Further research is needed to determine the mechanisms underlying the role of optimism in schema formation. Furthermore, research into the development of optimism could serve to broaden our resistance to unpleasant life events, much like an immunization against disease (Seligman 2002).

\section{Cross-References}

- Emotional Schema(s)

- Family Background and Effects on Learning

- Mental Model(s)

- Socialization-Related Learning

- Socio-Emotional Aspects of Learning

\section{References}

Bowlby, J. (1980). Attachment and loss. New York: Basic Books. Buri, J. R., \& Gunty, A. M. (2008, August). Family functioning and maladaptive schemas: The moderating effects of optimism. Paper presented at the 116th annual convention of the American Psychological Association, Boston.

Maccoby, E. E. (2007). Historical overview of socialization research and theory. New York: Guilford.

Piaget, J. (1954). The construction of reality in the child. Oxford: Basic Books.

Seligman, M. E. P. (2002). Authentic happiness. New York: Free Press. Walsh, F. (2006). Strengthening family resilience (2nd ed.). New York: Guilford.

Young, J. E. (1999). Cognitive therapy for personality disorders: A schema-focused approach (3rd ed.). Sarasota: Professional Resource.

\section{Early Schemas}

- Early Maladaptive Schemas: The Moderating Effects of Optimism 


\section{Ebbinghaus, Hermann (1850-1909)}

Norbert M. SeEL

Department of Education, University of Freiburg, Freiburg, Germany

\section{Life Dates}

Herman Ebbinghaus (Barmen, Germany, January 24, 1850 - Halle, Germany, February 26, 1909) was born to Lutheran merchants. He studied philosophy at the University of Bonn (and for a short time also at the universities of Berlin and Halle). He completed his dissertation on Eduard von Hartmann's Philosophy of the Unconscious and received his doctorate in 1873 at the age of 23. From 1873 to 1880, Ebbinghaus studied and worked as a tutor at several universities in England and France. Then he lectured at the University of Berlin where he served as private lecturer until 1894. In 1890, he cofounded and edited the Zeitschrift für Psychologie und Physiologie der Sinnesorgane. From 1894 to 1909, he was professor at the University of Breslau but also taught at Halle. Ebbinghaus founded laboratories for psychological research at the Universities of Berlin and Breslau. He was the father of the eminent Neo-Kantian philosopher Julius Ebbinghaus.

\section{Contribution(s) to the Field of Learning}

- "Psychology has a long prehistory but only a short history" (Ebbinghaus 1908).

Ebbinghaus was a true pioneer in the area of research on memory and learning (Wozniak 1999). He developed the first scientific approach to the study of higher psychological processes and was the first

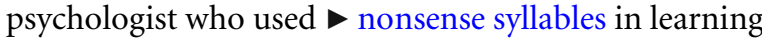
and memory research. His experiments demonstrated that meaningless stimuli are more difficult to memorize than meaningful stimuli; his experiments also revealed the interesting result that increasing the amount of material to be learned usually significantly increases the amount of time it takes to learn the material.

In 1885, Ebbinghaus published his groundbreaking Über das Gedächtnis (translated to English as Memory. A Contribution to Experimental Psychology), in which he described experiments he conducted to investigate the processes of learning and forgetting (Ebbinghaus 1885/ 1962). Several findings from these experiments are still relevant and valid today. His most famous finding by far is the forgetting curve, an exponential curve that illustrates how fast humans tend to forget the information they have learned. The sharpest decline is in the first $20 \mathrm{~min}$, then in the first hour, and then the curve evens off after about 1 day (Fig. 1).

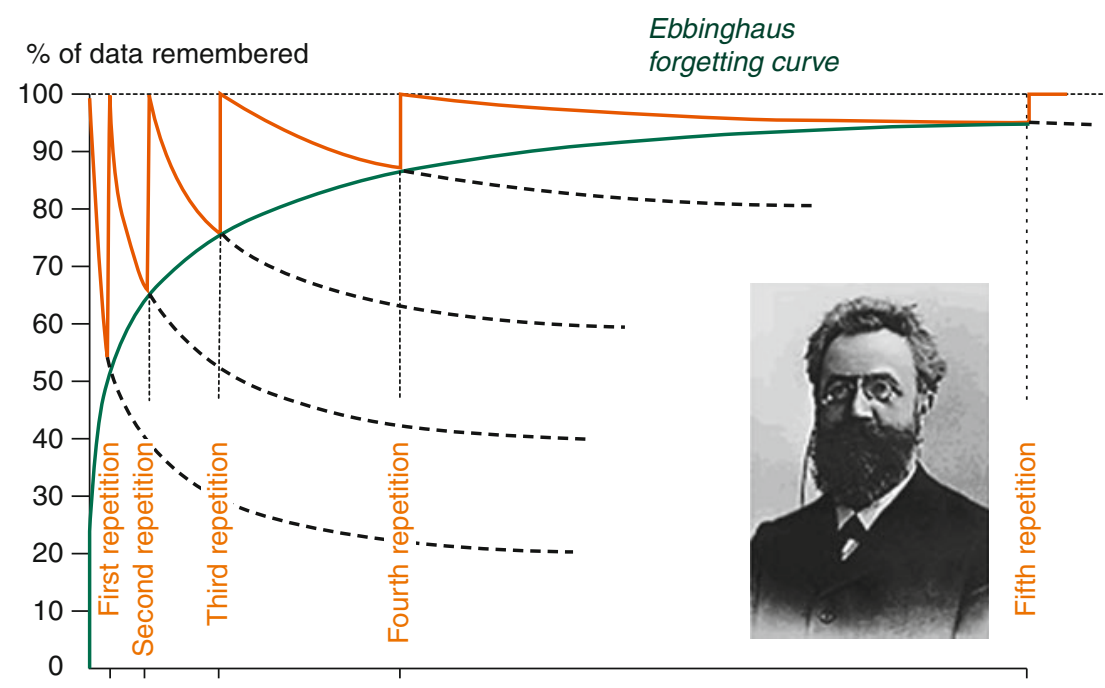

Ebbinghaus, Hermann (1850-1909). Fig. 1 The forgetting curve (Source: Sousa 2006, p. 99) 
Ebbinghaus' learning curve refers to how fast individuals learn information. The sharpest increase occurs after the first repetition but then gradually evens out due to the fact that less and less new information is retained after each repetition. Like the forgetting curve, the learning curve is also exponential. However, Ebbinghaus stated that relearning is easier than initial learning and that it takes longer to forget material after each subsequent relearning. Interestingly, his experiments also showed that learning is more effective when it is spaced out over time rather than crammed into a single extensive study session. Closely related to relearning is the concept of savings, which refers to the amount of information retained in the subconscious even after it has been forgotten and cannot be recalled. This can be illustrated by memorizing a list of items until perfect recall and then ceasing to recall any of the items for a time. When relearning the list and comparing the new learning curve to the learning curve of the previous memorization, we can observe that the second list is generally memorized faster. The difference between the two learning curves is what Ebbinghaus called "savings." Finally, Ebbinghaus also described the difference between involuntary and voluntary memory. Involuntary memory is associated "with apparent spontaneity and without any act of the will" whereas voluntary memory means bringing stored information "into consciousness by an exertion of the will."

Another effect documented by Ebbinghaus is the serial position effect, which describes how the position of an item in a list to be learned affects the likelihood that an individual will be able to recall it. Similarly to the forgetting and learning curve, Ebbinghaus introduced the serial position curve to illustrate the recency and primacy effect. The recency effect refers to the fact that individuals remember more recent information better due to short-term storage in memory, whereas the primacy effect refers to the fact that it is easier to remember the first items in a list due to increased - rehearsal and commitment to long-term memory.

\section{Important Scientific Research and Open Questions}

Most contributions to the scientific understanding of memory and learning prior to Ebbinghaus focused on introspection and observational description, mostly undertaken by philosophers such as Immanuel Kant or Sir Francis Bacon. The effects of Ebbinghaus's memory research were thus almost immediate. Although Ebbinghaus is generally considered to have popularized experiments in psychology, it is important to note that he was not the first psychologist to conduct experiments. Nevertheless, he can be credited with pioneering experimental research on memory and learning as well as sentence completion exercises, which he developed as part of his studies on children's cognitive abilities. In 1896, he realized a form of sentence completion test with schoolchildren in order to investigate the effects of exhaustion on cognitive achievement. He asked teachers to evaluate children's abilities and assigned the results to three different levels. He found significant relationships between the test results and the teachers' evaluations without the help of correlation analysis, which was unknown at this time. This was possibly the first test of verbal intelligence of subjects. Later, the same procedure was used by Alfred Binet. Since the time of Ebbinghaus, sentence completion has also been used extensively in memory research and in psychotherapy. In addition, it also influenced Charlotte Bühler's studies on language meaning and society.

From the perspective of philosophy of science, Ebbinghaus changed the dominant philosophical paradigm for research on memory and learning and illustrated that higher cognitive processes can be studied experimentally. Therefore, Ebbinghaus can be considered as a pioneer of the experimental methodology within the realm of psychology. Some of his innovations, such as the use of nonsense syllables, are still valuable tools in twenty-first-century learning and memory research.

\section{Cross-References}

- History of the Sciences of Learning

- Memory Persistence

- Retention and Learning

\section{References}

Ebbinghaus, H. (1885/1962). Memory: A contribution to experimental psychology. New York: Dover.

Ebbinghaus, H. (1908/1973). Psychology: An elementary textbook. New York: Arno Press.

Sousa, D. A. (2006). How the brain learns (3rd ed.). Thousand Oaks: Corwin Press.

Wozniak, R. H. (1999). Introduction to memory: Hermann Ebbinghaus (1885\&sol;1913). In R. H. Wozniak (Ed.), Classics in psychology, 1855-1914: Historical essays. Bristol: Thoemmes Press. 


\section{Ecology of Learning}

Reuven Dukas

Department of Psychology, Neuroscience \& Behaviour, Animal Behaviour Group, McMaster University, Hamilton, ON, Canada

\section{Definition}

Ecology is the study of interactions between organisms and their surrounding biotic and abiotic environments. The ecology of learning means an examination of the information animals learn and its effects on their interactions with physical factors such as temperature and topography, and biotic factors including other individuals, plants, prey, and predators in their environment.

\section{Theoretical Background}

Most animals are capable of learning, meaning that they can acquire neuronal representations of new information. Examples for learning include neuronal representations of new (a) spatial environmental patterns, (b) sensory information such as visual, auditory, or olfactory stimuli, (c) associations between stimuli and environmental states, and (d) motor patterns, for example, the sequence of body movements involved in successfully extracting food from inside the hard shells of nuts. Older analyses of animal behavior assumed that a large part of animals' behavioral repertoire was guided by genetically determined instincts. It is now, however, widely accepted that most animals, including tiny and short-lived ones such as fruit flies (Drosophila spp.), can learn (Dukas 2008). Thus learning can be perceived as a key animal characteristic that affects individuals' success in surviving and reproducing.

The most basic mechanisms allowing organisms to change their behavior with experience involve behavioral and genetic modulation. An example for behavioral modulation is chemotaxis, which allows bacteria and other organisms to move up food gradients and away from noxious compounds. That is, bacteria continuously monitor their external environment during movement. If their current surrounding is better than before, they keep moving in the same direction. If their current environment is worse than before, they tumble in order to change their direction of movement
(Eisenbach and Lengeler 2004). A classic example for genetic modulation is the lac operon. Bacteria such as Escherichia coli prefer to metabolize glucose over lactose. When glucose is not available, however, they can activate a set of genes that code for enzymes that enable them to rely on lactose to meet their energetic needs. In short, behavioral and genetic modulation are fundamental biological properties that allow organisms to respond adaptively to environmental variation and needs. Learning can be perceived as an extension of such modulation, which, through modulation of neurons, allows for the representation of new information. The neuronal modulation generating learning involves short- and long-term changes in synaptic properties mediated by neurotransmitters and gene expression (Keene and Waddell 2007).

What are the benefits of learning over innate behavior? Many environmental features are unique to a certain time and place. Animals' ability to learn about such features expands the type and amount of information they can respond to and increases their behavioral repertoire. For example, a bee can acquire a neuronal representation of the location of her nest in relation to prominent landmarks, record the spatial location, odor, and color of the preferred flowers in its specific area and time of year, and learn a new motor pattern for collecting nectar and pollen from these flowers.

\section{Important Scientific Research and Open Questions}

To date, the simplest animal known to express associative learning is the tiny soil nematode, Caenorhabditis elegans. This classical model system is $1 \mathrm{~mm}$ long and has about 950 cells, of which 302 are neurons (Ardiel and Rankin 2010). This indicates that even very small neuronal networks can generate associative learning. The ecology of learning, however, is better understood in another model system, the fruit fly, D. melanogaster. Fruit flies are rather small, about $2.5 \mathrm{~mm}$ long, and the adult brain contains about 100,000 neurons. Both the adult and larval stages of fruit flies feed on yeast grown on decaying fruits. Because female fruit flies already lay their eggs on the best food they can locate, the emerging larvae merely have to commence feeding, which is their sole task. Even though the larval task seems easy and they possess limited sensory and information processing abilities, the larvae show robust associative 
learning within the only two domains relevant to their life stage. First, the larvae can learn to prefer odors or illumination conditions (light or dark) indicating better quality food among distinct alternatives, and, second, the larvae can learn to avoid odors associated with danger.

Like the larvae, adult fruit flies can learn to prefer odors associated with food and to avoid odors associated with danger. The adults can also learn to avoid light sources of distinct frequencies associated with aversive states (shock or violent shaking) and to avoid flying toward visual patterns associated with high temperature. In short, adult fruit flies can learn about odors, colors, and visual patterns associated with either food or danger. Much of the adult life, however, is concerned with reproduction. Traditional accounts of insect reproductive behavior assumed that such behavior is innate. Indeed, some aspects of fruit fly sexual behavior are considered to be fully under genetic control. For example, an important component of male courtship is the 'love song', generated through wing vibration. The females carefully evaluate the male's song and reject males who do not meet strict speciesspecific and quality criteria. Current evidence indicates that the execution of the males' song is not affected by learning. The males, however, do show adaptive learning in the context of courtship.

To maximize their reproductive success, the males have to focus their courtship efforts on the females who are most likely to accept them as mates. A major feature identifying such females is their pheromonal profile. Female pheromones, however, greatly vary over time, space, and females' ages and reproductive states. Although males possess some evolved preferences for certain female pheromonal blends, the variation in these pheromones limits the effectiveness of such innate preferences. Instead, the males initially court a wide variety of females and gradually learn to avoid the female categories that consistently reject them. Through such associative learning, the males can quickly maximize the time they devote to the most receptive females (Dukas 2009).

Like the males, the females show adaptive learning in the context of mate choice. The females should attempt to mate with the best available male, but male variation in time and space implies that the females would gain from adjusting their mate choice criteria based on their knowledge about the locally available males. Prior to reaching sexual maturity, females are courted vigorously by numerous males. Females that encounter only less attractive, small males during that period, are more likely to later accept small males as mates than females who have previously encountered large males. Female fruit flies can also learn the odor and color features associated with preferred egg-laying substrates. Naive females' choices of egg-laying substrates are even influenced by the choice of experienced females. That is, the females show basic social learning abilities (Sarin and Dukas 2009).

The above review of fruit fly learning illustrates how ubiquitous and important learning is even in animals with very small brains and short life spans. The slightly larger, yet short-lived, honey bees (Apis mellifera) further demonstrate the remarkable capacity of a small brain, which, in the case of honey bees, contains about one million neurons. In addition to learning about food and danger, honey bees show exceptional spatial learning, which allows them to fly to flowers over $10 \mathrm{~km}$ away from the hive and find their way home. Honey bees also possess motor learning, which enables them to learn how to manipulate flowers with complex morphology and thus increase the rate at which they collect nectar and pollen from flowers (Dukas 2009).

Longer-lived animals with much larger brains than insects can accumulate vast amounts of information with experience. In species with parental care, individuals often spend a significant proportion of their lifetime learning under their parental guidance. Learning typically starts just after birth, at which time the newborn are imprinted to their parents. Imprinting occurs during a critical time period early in life at which animals may learn visual, vocal, and scent characteristics of their parents. This information allows young individual recognition of their parents and later would determine their preferences for sexual partners.

In sum, learning allows individuals to acquire information specific to their time and place. It enables them to choose among alternative food sources, to avoid places and other cues of danger, to spatially orient in their surroundings, to learn about other individuals including their parents, siblings and neighbors, and to accumulate knowledge throughout their life either through their own experience or via social learning. Such knowledge makes experienced individuals experts in their domain. Expertise translates into a gradual increase in performance on relevant ecological tasks, 
for example, the daily rate of food collection in forager honey bees, and the annual rate of offspring production in birds (Dukas 2009).

\section{Cross-References}

Evolution of Learning

- Expertise

- Habituation

- Learning and Instinct

- Sensitization

- Social Learning in Animals

\section{References}

Ardiel, E. L., \& Rankin, C. H. (2010). An elegant mind: Learning and memory in Caenorhabditis elegans. Learning \& Memory, 17, 191-201.

Dukas, R. (2008). Evolutionary biology of insect learning. Annual Review of Entomology, 53, 145-160.

Dukas, R. (2009). Learning: mechanisms, ecology and evolution. In R. Dukas \& J. Ratcliffe (Eds.), Cognitive ecology II. Chicago: University of Chicago Press.

Eisenbach, M., \& Lengeler, J. W. (2004). Chemotaxis. London: Imperial College Press.

Keene, A. C., \& Waddell, S. (2007). Drosophila olfactory memory: Single genes to complex neural circuits. Nature Reviews. Neuroscience, 8, 341-354.

Sarin, S., \& Dukas, R. (2009). Social learning about egg laying substrates in fruit flies. Proceedings of the Royal Society of London B-Biological Sciences, 276, 4323-4328.

\section{Ecopsychology}

- Nature-Guided Therapy

\section{Ecotherapy}

Nature-Guided Therapy

\section{Educating Robots, Teaching Robots as Humans}

Cognitive Artifacts and Developmental Learning in a Humanoid Robot

\section{Education}

- Affective and Cognitive Learning in the Online Classroom

$\checkmark$ Learning at Play

\section{Education analytics}

Educational Data Mining

\section{Education of Facilitators (For Educators of Practicing Teachers)}

- Education of Teacher Educators

\section{Education of Professional Development Providers}

- Education of Teacher Educators

\section{Education of Teacher Educators}

Ruhama Even

Department of Science Teaching, Weizmann Institute of Science, Rehovot, Israel

\section{Synonyms}

Education of facilitators (for educators of practicing teachers); Education of professional development providers; Education of teachers of teachers; Preparation of teacher educators

\section{Definition}

Education of teacher educators refers to the preparation, teaching, or facilitating of teacher educators. 
Nowadays, the term "teacher educators" commonly refers to both those who educate prospective teachers and those who educate practicing teachers, that is, to those who initiate, guide, and support teacher learning across the lifespan. Yet, sometimes the term "teacher educators" refers only to educators of prospective teachers, that is, to those who teach future teachers, and not to those who provide professional development for practicing teachers.

\section{Theoretical Background}

There is general recognition and agreement today that the education and professional development of teachers is key to improving students' opportunities to learn. Accordingly, the focus and nature of the education of prospective and practicing teachers have received immense international attention in recent years, and the past decades have seen substantial increase in scholarship on teacher professional education. A significant issue identified recently as crucial for improving the education and professional development of teachers is the education and development of teacher educators (Even and Ball 2009; Jaworski and Wood 2008; Russell and Korthagen 1995).

In different countries around the world, various professionals are responsible for initiating, guiding, and supporting teachers' learning: university faculty with disciplinary expertise and those who specialize in education; school teachers, teacher mentors, and staff of curriculum implementation projects; educators whose major occupation is to work with teachers and those who do it only as an add-on part-time temporary activity; those who work with both prospective and practicing teachers; and those whose role is to educate solely prospective or practicing teachers, but not both. Yet, this vast range of teacher educators has little formal preparation for their work. Most become teacher educators through practice with little institutional and professional support.

With the expanding current interest in the issue of professional education and development of teacher educators in different countries, pioneering formal programs to prepare educators to educate teachers started to emerge. These include, for example, the Leadership Curriculum for Mathematics Professional Development (LCMPD) Project in the United States, the MANOR Program and the MOFET Institute in Israel, and a special M.Ed. Programme in Pakistan.

\section{Important Scientific Research and Open Questions}

The education of teacher educators has only recently become of interest to the international community. Thus, not much is known about the development of teacher educators, and about effective ways to educate educators to initiate, guide, and support teacher learning. Some of the current research on the professional education and development of teacher educators approach this issue as general with no attention to subject matter (Cochran-Smith 2003; Russell and Korthagen 1995; Swennen and van der Klink 2009). Other research studies center on issues pertaining to professional education and development of teacher educators in a specific subject area - for example, in mathematics (Even 2005; Jaworski and Wood 2008).

Most research on the professional education and development of teacher educators includes reflections of teacher educators on their own personal development (e.g., Cochran-Smith 2003; Jaworski and Wood 2008; Russell and Korthagen 1995; Swennen and van der Klink 2009). This research suggests that reflective inquiry has a central role in learning to teach teachers and in developing as teacher educators. Yet, this line of research provides information mainly on the professional development of university-based teacher educators, but not on that of the wide range of professionals responsible for supporting prospective and practicing teachers' learning.

Because formal preparation for teacher educators scarcely exists, research that examines formal programs and activities intended to educate teacher educators is sparse. Pioneering work in this direction addresses various aspects of curriculum (What should teacher educators learn?) and pedagogy (How should teacher educators be taught?). It suggests several areas of professional knowledge base for teacher educators (Jaworski and Wood 2008); two relate to knowledge shared by teacher educators and teachers: pedagogical knowledge and disciplinary knowledge. A third area of professional knowledge base for educating teacher educators relates to knowledge specific to the teacher educator: knowledge of teaching teachers and of teachers' learning. In addition to professional knowledge base, research suggests the need to purposely teach practices of educating teachers, giving explicit attention to the nature of work in which teacher educators engage. These practices may be general, such as, teaching 
courses, supervising student teachers, facilitating seminars (Cochran-Smith 2003), or subject matter specific, such as, planning, conducting, and assessing activities, workshops, and courses for mathematics teachers (Even 2005). This line of research also suggests that inquiry is central to learning to teach teachers and to developing as teacher educators. Additionally, it shows the importance of attending to the relationships of knowledge and practice.

Thus far, it is not known whether, or in what ways, formal education of teacher educators needs to be responsive to the wide range of professionals responsible for supporting teachers' learning or may be common to all. For example, whether the professional education of educators of practicing teachers needs to be different from the education of educators of prospective teachers, as the education of prospective and that of practicing mathematics teachers are commonly of different nature, often occurring in different settings, and not necessarily conducted by the same people.

\section{Cross-References}

- Inquiry Learning

- Professional Learning and Development

\section{References}

Cochran-Smith, M. (2003). Learning and unlearning: The education of teacher educators. Teaching and Teacher Education, 19, 5-28.

Even, R. (2005). Integrating knowledge and practice at MANOR in the development of providers of professional development for teachers. Journal of Mathematics Teacher Education, 8(4), 343-357.

Even, R., \& Ball, D. L. (Eds.). (2009). The professional education and development of teachers of mathematics - the 15th ICMI Study. New York, NY: Springer.

Jaworski, B., \& Wood, T. (Eds.). (2008). The international handbook of mathematics teacher education: The mathematics teacher educator as a developing professional. Rotterdam, The Netherlands: Sense.

Russell, T., \& Korthagen, F. (Eds.). (1995). Teachers who teach teachers: Reflections on teacher education. London: Falmer Press.

Swennen, A., \& van der Klink, M. (Eds.). (2009). Becoming a teacher educator. Dordrecht: Springer.

\section{Education of Teachers of Teachers}

- Education of Teacher Educators

\section{Education Permanente}

- Lifelong Learning

\section{Educational Data Mining}

\section{Synonyms}

Analysis of learning data; Education analytics; e-Learning data analysis

\section{Definition}

Computer-based learning systems can now keep detailed logs of user-system interactions, including key clicks, eye-tracking, and video data, opening up new opportunities to study how students learn with technology. Educational Data Mining (EDM; Romero et al. 2010) is concerned with developing, researching, and applying computerized methods to detect patterns in large collections of educational data - patterns that would otherwise be hard or impossible to analyze due to the enormous volume of data they exist within. Data of interest is not restricted to interactions of individual students with an educational system (e.g., navigation behavior, input to quizzes and interactive exercises) but might also include data from collaborating students (e.g., text chat), administrative data (e.g., school, school district, teacher), and demographic data (e.g., gender, age, school grades). Data on student affect (e.g., motivation, emotional states) has also been a focus, which can be inferred from physiological sensors (e.g., facial expression, seat posture, and perspiration). EDM uses methods and tools from the broader field of Data Mining (Witten and Frank 2005), a sub-field of Computer Science and Artificial Intelligence that has been used for purposes as diverse as credit card fraud detection, analysis of gene sequences in bioinformatics, or the analysis of purchasing behaviors of customers. Distinguishing EDM features are its particular focus on educational data and problems, both theoretical (e.g., investigating a learning hypothesis) and practical 
(e.g., improving a learning tool). Furthermore, EDM makes a methodological contribution by developing and researching data mining techniques for educational applications. Typical steps in an EDM project include data acquisition, data preprocessing (e.g., data "cleaning"), data mining, and validation of results.

\section{Theoretical Background}

Historically, EDM is a relatively new scientific discipline. Although researchers have been recording and analyzing data from educational software for a long time, only recently has EDM been established as a field in its own right, through conferences (Internal Conference on Educational Data Mining, started in 2008) and a scientific journal (Journal of Educational Data Mining (JEDM), first issue published 2009). EDM research is also presented and promoted at educational technology conferences, such as the International Conference on Artificial Intelligence in Education (AIED), the International Conference on Intelligent Tutoring Systems (ITS), and the International Conference on User Modeling, Adaptation, and Personalization (UMAP).

EDM borrows from and extends related fields such as Machine Learning (the study of computer programs that learn from and improve with empirical data), text mining (approaches to finding patterns in natural language text), and statistics. Other important influences are psychometrics (the study of psychological instruments to measure human skills and traits) and Web log analysis (approaches to identify user profiles and navigational patterns of Web site users).

EDM provides a rich toolbox of analysis techniques for a variety of problems in educational research and technology development (Romero et al. 2010; International Working Group on Educational Data Mining, n.d.):

- Scientific inquiry and system evaluation. EDM can contribute to the evaluation of learning systems and the development and testing of scientific theories on technology-enhanced learning (TEL). Exploratory analyses can be used to identify regular (or unusual) patterns in data, for instance, problemsolving strategies of students and patterns of successful and unsuccessful collaboration, thus helping to formulate new scientific hypotheses. EDM can be used to compare different interventions, for instance, how different types of practice compare to one another (e.g., in language learning, is it more efficient to reread the same stories or to read a variety of stories?). To simplify the execution of studies, EDM researchers developed computerized methods to randomize treatment assignment and to capture data. Finally, EDM researchers have developed new evaluation methods that are based on specific models of learning (e.g., learning curves and Bayesian Knowledge Tracing).

- Determining student model parameters. A student model is a data structure, typically used in $>$ Intelligent Tutoring Systems (ITS), that keeps track of relevant student characteristics over time (e.g., how a student's mastery of a particular skill improves with practice) through inferences from observable user actions (e.g., student answers to quiz questions). Student models allow systems to adapt to students and situations (e.g., selecting an exercise with an appropriate level of difficulty). Often, these inferences are based on parameterized probabilistic models. For instance, Bayesian Knowledge Tracing (Corbett and Anderson 1995) - a modeling approach frequently used to implement $\downarrow$ Mastery Learning - uses parameters to represent the probability that a student only guessed the correct solution. System developers are confronted with the problem of how to choose appropriate parameter values. One solution is to use EDM to estimate parameter values from real data.

- Informing domain models. Student models are typically built upon a domain model, that is, a model that formally describes the domain of instruction in terms of concepts, skills, learning items, and their interrelationships. To enable student models to accurately predict knowledge and skills, it is important that the domain model reflects aspects of human cognition, such as knowledge and problem-solving skills. EDM can help in the design, refinement, and evaluation of domain models. For instance, EDM has been used to induce domain structures from data, to detect empirically plausible knowledge components that were originally not included in a domain model, and to compare skill models with different granularities (e.g., finegrained models with many skills and coarse-grained ones with only a few skills).

- Creating diagnostic models. Learning systems typically diagnose student's progress to drive 
adaptation and $>$ feedback. Yet, the patterns these diagnoses are based on are often complex and/or not well understood. EDM provides tools to explore existing data to sharpen the understanding of patterns of interest and to induce diagnostic models from data. For instance, models have been developed to identify gaming-the-system behavior (students exploit properties of the learning system to "succeed," e.g., by excessive use of a hint function to get the answers to problems), students' learning styles, off-topic contributions in discussions, problems during collaboration, and students' emotions. Once such models have been developed, corresponding analysis results can be fed into a student model.

- Creating reports and alerts for instructors, students, and other stakeholders. It is usually difficult for teachers in computer-based learning scenarios to monitor their students' learning progress and problems in real time (or even shortly after) because of a lack of face-to-face interaction, differences in time and place, and the impracticality of immediate and rapid manual analysis of computer logs. EDM can be used to build teacher tools that employ statistics, visualizations, and other ways of representing information in an intelligible way to facilitate the exploration of data, to increase awareness of students' current learning, and to pinpoint possible problems that may require remediation. For instance, tools have been developed that provide statistics on content usage, student performance, and participation in collaborative activities, as well as visualizations of navigation paths and social networks. Similarly, such information can be provided to students to support awareness and $\boldsymbol{\nabla}$ metacognition.

- Recommending resources and activities. EDM can be used for adaptive instructional support by determining learning resources and activities that are appropriate with respect to a learner's needs, interests, preferences, skill level, and past activities. For instance, a resource could be recommended that has been used by other students with a similar profile who successfully mastered an intended learning goal before.

EDM uses a wide range of methods to analyze data. The following taxonomy builds upon previously proposed taxonomies (Romero and Ventura 2007; Baker and Yacef 2009):
- Supervised model induction comprises machine learning techniques that infer prediction models from training instances for which the values of a target attribute are known. Prediction models accept instances as input (typically described as an attribute vector) and output a prediction for the target attribute. Models that predict categorical target values are called classification models; models that predict continuous target values are called regression models. Prediction models can be based on different representations, for instance, Decision Trees, Support Vector Machines (both classification), and linear regression model (regression). An example application in EDM is the categorization of discussion contributions into on-topic and offtopic contributions (the target attribute) based on the list of terms extracted from the contribution's text (the attribute vector).

- Unsupervised model induction comprises machine learning techniques that infer models from training instances for which the values of a target attribute are not known. Unsupervised methods use a bottom-up approach, that is, patterns and structures are searched in the input space without explicitly defined target categories or labeled examples. A widely used approach is clustering, which is used to identify groups of instances in a training set that are "similar" in some respect. Typically, some kind of distance measure (e.g., Euclidian distance) is used to decide how similar instances are. Once a set of clusters has been determined, new instances can be classified by determining the closest cluster. One well-known clustering algorithm is k-means clustering. Example applications in EDM are the identification of similar course materials or similar interaction sequences in $>$ collaborative learning.

- Parameter estimation comprises statistical techniques to infer parameters of probabilistic models from given data. These models can be used to predict the probability of events of interest. The approach is based on the assumption that the model has a given parametric form (e.g., a Gaussian distribution with the parameters mean and variance). An example application in EDM is the estimation of Bayesian Knowledge Tracing (BKT) parameters. BKT is used to determine the probability that a student has mastered a skill based on the history of past performances. A BKT model 
can be understood as a Dynamic Bayesian Network with four parameters (prior, guess, slip, and learn rate). These parameters can be determined, for instance, with the Expectation-Maximization algorithm. Besides parametric estimation methods, there are also nonparametric methods that do not assume a specific parametric form.

- Relationship mining is concerned with the identification of relationships between variables - relationships which might be associative, correlational, sequential, or causal in nature. For instance, a common approach to association rule mining is to learn IF-THEN rules that exceed a minimum "support" and "confidence" threshold. Support denotes the relative frequency of transactions that match both the IF and THEN part of the rule. Confidence denotes the relative frequency of transactions that match the THEN part of the rule within the set of transactions that match the IF part. Apriori is, for instance, a classical association rule algorithm. An example application in EDM is the identification of mistakes that frequently occur together (e.g., students who made the mistakes $A$ and $B$ also often made mistake $C$ ).

- Distillation of data for human judgment aims at representing data in intelligible ways using statistics, visualization methods, and interactive information interfaces. For instance, average performance scores can be computed for each student and presented to a teacher in ascending order in a bar chart. Another example is learning curves, which plot a student's performances (e.g., response time) against the number of opportunities of practicing a skill. An ideal learning curve shows that performance improves smoothly and monotonically, approximately following a power law or exponential function. On the other hand, learning curves with spikes indicate that another skill might interfere with the actually modeled skill, that is, the skill model could be improved.

- Discovery with models comprises approaches that bootstrap already existing models to make discoveries rather than computing new models from scratch. For instance, a prediction model could be applied to a data set to predict the values of a target category of interest. The predictions themselves could be used as data in other analyses again, for instance, they could be correlated with a target category of another prediction model. Another example is to scrutinize the different components of an existing prediction model to learn about factors that influence the prediction (e.g., how do interaction sequences of successful and failed knowledge sharing in collaborative learning differ?).

\section{Important Scientific Research and Open Questions}

A typical characteristic of educational data is its nonindependence. For instance when we collect data from education discussions and want to classify whether contributions are on-topic or off-topic, we have to consider that contributions are not statistically independent of one another since multiple contributions might stem from the same student or discussion. This might harm the computation of models (standard machine learning schemes typically have the built-in assumption of independent training examples) as well as the validation of models (e.g., a cross-validation might lead to biased results when training and test set are not independent).

Results from EDM research are typically achieved in the narrow context of specific research projects and educational settings (e.g., a particular school). The question arises how general such results are, for instance, whether the same student model parameters also can be used with other student populations, or whether a predictive model is still reliable when used in a different context. Therefore, there is an increasing need for replication studies to test for broader generalizations.

As a practical consequence of this need, EDM researchers have become increasingly more interested in open data repositories and standard data formats to promote the exchange of data and models. An example is the PSLC DataShop (http://pslcdatashop.org), a repository for educational data that has been opened to the EDM community, which provides data import and export facilities as well as analysis and visualization tools.

\section{Cross-References}

- Advanced Learning Technologies

- Computer-Enhanced Learning and Learning

Environments

- Design of Learning Environments 
Learning Criteria, Learning Outcomes, and Assessment Criteria

- Probability Theory in Machine Learning

- Supervised Learning

- Unsupervised Learning

\section{References}

Baker, R., \& Yacef, K. (2009). The state of educational data mining in 2009: a review and future visions. Journal of Educational Data Mining (JEDM), 1(1), 3-17.

Corbett, A. T., \& Anderson, J. R. (1995). Knowledge tracing: modeling the acquisition of procedural knowledge. User Modeling and User-Adapted Interaction, 4(4), 253-278.

International Working Group on Educational Data Mining. (n.d.). Educational Data Mining Home Page. http://www.educationaldatamining.org. Accessed 23 December 2010.

Romero, C., \& Ventura, S. (2007). Educational data mining. a survey from 1995 to 2005. Expert Systems with Applications, 33(1), 135-146.

Romero, C., Ventura, S., Pechenizkiy, M., \& Baker, R. S. J. D. (Eds.). (2010). Handbook of educational data mining. Boca Raton, FL: CRC Press.

Witten, I. H., \& Frank, E. (2005). Data mining: Practical machine learning tools and techniques (2nd ed.). San Francisco: Morgan Kaufmann.

\section{Educational Experiments}

- Field Experiments in Learning Research

\section{Educational Games}

Games-Based Learning

\section{Educational Gaming}

Edutainment and Learning

\section{Educational Neuroscience}

- Developmental Cognitive Neuroscience and Learning

- Neuroeducational Approaches on Learning

\section{Educational Objectives}

- Learning Objectives

- Outcomes of Learning

\section{Educational Software}

- Courseware Learning

\section{Educational Technology}

- Everyday Learning: Instruction and Technology Designs

\section{- Learning Technology}

\section{Educational Television}

- Children's Learning from Television

\section{Educational Use of Visualization and Animation}

Visualizations and Animations in Learning Systems

\section{Educational-Military-Industrial Complex}

\section{Educational Linguistics}

Linguistic Factors in Learning
- Reproductive Learning 


\section{Education-Entertainment}

- Children's Learning from Television

\section{Edutainment}

Learning with Games

\section{Edutainment and Learning}

\section{ZUHAL OKAN}

ELT Department, Çukurova University, Balcali, Adana, Turkey

\section{Synonyms}

Educational gaming; Fun learning; Infotainment; Technotainment

\section{Definition}

"Edutainment" is a hybrid genre combining learning and fun. It relies heavily on visual material, on narrative or game-like formats, and on more informal, less didactic styles of address (Buckingham and Scanlon 2000). The purpose of edutainment is to attract and hold the attention of the learners by engaging their emotions through a computer monitor full of vividly colored animations. Similarly, "edutainment" suggests overtly entertaining learning materials, which contain messages addressed to both parents and children. Through explicit educational claims, edutainment software encourages the parents to believe that this software is beneficial in developing children's skills in a variety of subjects. They also raise learners' expectations that learning can be enjoyable and fun.

\section{Theoretical Background}

Although the interest in edutainment software has surged in the early 1990s, the idea of combining entertainment and education goes back to 1970s when first video games were marketed. Inspired by behaviorism first and then cognitivism and constructivism, educators have been increasingly incorporating various games into their teaching curriculum in an effort to create a fun and engaging learning environment for students.

Without a doubt computer technology holds great potential for improving the way that people learn. Through the use of network resources, learners can engage in individualized instruction where they can investigate and learn concepts and content to meet their specific needs and have fun at the same time. In recent years, the shift in educational philosophy from traditional teacher-centered instruction to a more interactive, engaging, experiential method has led John Dewey and other theorists to incorporate cognitive psychology into their theories of education. They pointed out the importance of initiative and excitement on the part of the child in learning and emphasized learning as a lifelong activity fundamental to human existence.

Supplemented by a constructivist perspective, Kolb's experiential learning theory also gives theoretical support for integration of game play and pedagogy. Based on the work of Piaget, Lewin, and Dewey, - experiential learning emphasizes an active knowledge construction process involving transactions between a person and the environment. In this process, prior experiences direct a person's way of thinking and knowledge construction. This links well with the recent educational reform efforts that considered "play" as a site of learning. It is identified as a process through which children acquire various skills such as self-reflection, problem-solving, and abstract thinking while, at the same time, learn to manage their emotions, test their limits, and develop complex communication skills.

The increasing use of technology has not only changed the form and nature of play, it has put gaming at the center of children's lives. Similarly, educational software designed to mediate between entertainment and education has come to appear more frequently in both educational settings and at home. It has been recognized that such software has the potential to support new forms of active learning such as "learning by doing" rather than being told what to do.

Perhaps the most widely articulated argument for the use of edutainment materials is that such software motivates students to explore topics in greater depth. Supported by Malone's (1981) theory of $\boldsymbol{\nabla}$ intrinsic 
motivation which is a function of an optimal relationship between challenge, fantasy, curiosity, and control, it is asserted that because students are highly motivated through rich, interesting, and engaging learning experiences, their understanding of the subject is enhanced. Moreover, their senses are enthralled and stricken on all levels. Therefore, students cannot help but pay attention to information that is presented in dynamic and memorable ways, so learning becomes an incidental part of the activity. Additionally, it is argued that when students are having fun, they are motivated to persist for longer periods of time or to approach the learning activity more often.

It is tacitly assumed that one of the most powerful features of such software is its capacity to individualize instruction to meet the specific needs of the learner and, therefore, enable a more flexible pace of learning. Learners can choose from a variety of educational software packages offering self-paced instruction, content presented in a variety of ways (e.g., text, video, sound, graphics), in realistic and stimulating learning environments. However, these same features can also cause problems for some students because they may feel overwhelmed with too many choices. For example, they may be bombarded with non-linear structure and not know where to navigate next (EgenfeldtNielsen 2005). Therefore, the value of hypermedia learning depends on each individual and an understanding of learners' individual differences arguably becomes an important consideration in hypermedia learning.

In addition, the development of new technologies poses a specific challenge for the use of verbal and pictorial information in learning and instruction. Edutainment software puts special emphasis on the use of visuals to make the learning an immersive experience where the learner uses all her/his senses. Based on Paivio's dual coding theory, it is argued that welldesigned multimedia, combining instructional text and images, can enhance learning by taking advantage of humans' separate information processing channels for verbal and visual material (Mayer and Moreno 2003). Thus, students are better able to build mental connections between corresponding words and pictures when both are presented (i.e., animation and narration) than when only one is presented (i.e., narration) and the learner must mentally create the other.

\section{Important Scientific Research and Open Questions}

Despite the widespread enthusiasm for using edutainment software in educational settings, there are numerous studies examining critically its educational potential together with the advantages and disadvantages it might bring to the instructional process.

One often cited concern is the pedagogic approach behind edutainment software that emphasizes fun and enjoyment, often at the expense of educational content. Learners who are exposed heavily to the Internet, video games, and ready-made images presented by multimedia develop a new attitude toward learning. They come to equate learning with fun suggesting that if they are not enjoying themselves, they are not learning. In other words, learning becomes an obstacle that learners need to overcome. Thus, they prefer tasks that are fast, active, and exploratory with multiple and parallel channels of information.

Similarly, Salomon (1998) argues that learning is highly affected by the way one perceives the source of the information-to-be-learned and by the amount of mental effort learners invest in processing the material. Technology is a great tool for accessing information, but when it comes to performing the more important task of transforming the new information into knowledge, it must occur within the learners themselves. Salomon, when he assesses the use of technology in a constructivist-learning environment, draws attention to the negative effects of learning by means of multimedia and hypermedia. He argues that hypermedia and the Internet have a non-linear, association-based structure, and the learner, lured by the visual appeal of the presentation, wanders from one item to the other. In fact, as Healey (1998) notes, one of the important learning skills threatened by electronic stimulation is selective attention: the ability to direct one's attention and focus clearly on what is to be learned without succumbing to distraction. Children, when faced with flashy special effects, cannot resist the temptation to click impulsively, which encourages stimulus-bound behavior (Healey 1998). In fact, many educators and parents complain about students having short attention spans and lacking critical thinking skills.

The assumption exists that instructional designs when combined with fun elements enhance learning. However, if the co-existence of education and entertainment within learning environments is to be 
implemented, as in the case of edutainment software, the question is how much "edu" and how much "tainment" (Mann 1996) should be included. In fact it is agreed that too much simulation can produce a very dry and often frustrating learning experience and distract from the learning, making the users feel like they are mindlessly following directions.

As for the common discourse of "strong motivation qualities of edutainment software for all learners," Egenfeldt-Nielsen (2005) states edutainment holds little intrinsic motivation; rather, he asserts, it relies more on extrinsic motivation through rewards (i.e., getting points for completing a level). Here the fundamental question is to relegate motivation to merely making learning fun through such sensory-rich learning environments which not only weakens the role of motivation in education, but also underestimates the complex relationship between motivation and cognition. Rather, for motivation to serve as an agent of cognition requires that it should direct students to initiate learning activities and maintain an involvement in learning as well as a commitment to the process of learning. Furthermore, there is lack of data based research showing the motivating impact of such materials on various types of learners differing in terms of their existing knowledge and experience, their style of learning, and their motives to use the software.

Edutainment software puts special emphasis on the design and use of verbal and pictorial information in learning and instruction. Research on educational psychology, however, suggests that effective learning with visuo-spatial adjuncts is not dependent on the professional appearance of visuals, but rather on the relation between these displays and the task demands and on the learner's prior knowledge and cognitive abilities. Instructional design of visual displays, therefore, requires sufficient understanding of how the human cognitive system interacts with these displays. Thus, contrary to the widespread belief, the support that visual displays provide the learners is not automatic. In fact for learning to occur, there must be a relation between the displays and task demands, and on the learners' prior knowledge and cognitive abilities.

\section{Cross-References}

- Animation and Learning

- Computer-Enhanced Learning and Learning Environment
- Games-Based Learning

- Joyful Learning

- Metacognition and Hypermedia Learning - How Do They Relate?

- Multimedia Learning

- Play, Exploration, and Learning

\section{References}

Buckingham, D. \& Scanlon, M. (2000). That is edutainment: Media, pedagogy and the market place. Paper presented to the International forum of researchers on young people and the media, Sydney.

Egenfeldt-Nielsen, S. (2005). Beyond edutainment: Exploring the educational potential of computer games. Unpublished Ph.D. thesis, IT-University of Copenhagen, Copenhagen.

Healey, J. M. (1998). The "meme" that ate childhood. Education Week, Oct 7.

Malone, T. (1981). Towards a theory of instrinsically motivating instruction. Cognitive Science, 4, 333-369.

Mann, D. (1996). Serious play. Teachers College Record, 97(3), 446-470.

Mayer, R., \& Moreno, R. (2003). Nine ways to reduce cognitive load in multimedia learning. Educational Psychologist, 38, 43-52.

Salomon, G. (1998). Novel constructivist learning environments and novel technologies: some issues to be concerned with. Research Dialogue in Learning and Instruction, 1(1), 3-12.

\section{Effect of Fading Support}

Guidance-Fading Effect

\section{Effect of Intermittent Reinforcement}

Partial Reinforcement Effect

\section{Effectance Motivation}

- Understanding Intrinsic and Extrinsic Motivation: Age Differences and Meaningful Correlates 


\section{Effective learning}

- Active Learning

- Autonomous Learning and Effective Engagement

- Constructivist Learning

Evidence-Based Learning

\section{Effects of Anxiety on Affective Learning}

\author{
D. ERIK EverharT \\ Department of Psychology, East Carolina University, \\ Greenville, NC, USA
}

\section{Synonyms}

Auditory affective verbal learning (AAVL); State anxiety; Trait anxiety; Verbal emotional learning

\section{Definition}

Elevated levels of anxiety may alter performance across a wide range of tasks including physical and cognitive. For instance, it is well established that anxious individuals often display a bias for negative information. Likewise, individuals with elevated levels of anxiety display heightened physiological activity that may be manifested via increased heart rate and blood pressure, and an overall heightened state of arousal. More recently, the effects of anxiety on learning affective (i.e., emotional) words has been examined. The negative affect bias and heightened arousal may ultimately impact learning patterns with positive and negative words (Everhart et al. 2005).

\section{Theoretical Background}

Research indicates that high trait anxious subjects focus attention to negative threat words, and low trait anxious subjects avoid negative threat words. However, state anxiety mediates these results. The attendance to or avoidance of negative threat words is greater when participants are in a "state anxious" condition (Black et al. 2008). Anxious individuals also display a negative memory bias. For example, Foa and Kozak's (1986) cognitive theory of fear proposes that pathologically fearful individuals have greater perception, attention, and memory for stimuli related to their fear structure.

The effects of the negative information bias among individuals with elevated levels of anxiety on affective learning have been explored via the use of an affective verbal learning test. The Auditory Affective Verbal Learning Test (AAVL), as originally developed by Snyder and Harrison (1997), consists of two 15-item word lists. One word list is comprised of positive affective words, while the other is comprised of negative affective words. The word lists were developed using Toglia and Battig's (1978) index of word norms. Within this index of word norms, a total of 2,854 words were initially evaluated using a seven-point Likert Scale for concreteness, imagery, categorizability, meaningfulness, familiarity (FAM), number of attributes, and pleasantness (PLS). The positive affective words selected for the AAVL were the 15 words that had the highest mean PLS rating, while the negative affective words selected for the AAVL were the 15 words that had the lowest mean PLS rating. An additional criterion for word selection included a FAM rating of at least 5.0 or better. In general, primacy effects have been observed for the negative word list, while recency effects are observed for the positive word list. The AAVL has been utilized in numerous studies in order to examine learning patterns, peripheral physiological correlates, and neurophysiological correlates of a wide range of psychopathology including hostility and neurological disorder.

Individuals with elevated state anxiety display diminished recall of positive emotional words in the primacy position during the first learning trial of the AAVL. Elevated state anxiety is also associated with recall of fewer words within the recency position on the first learning trial of the negative word list (Black et al. 2008). One conclusion is tentatively drawn from review of the neuroanatomical mechanisms. First, historically, the primacy effect has been described as reflective of "long-term" encoding processes. The reduced primacy effects observed among high anxious individuals for the positive word list of the AAVL are possibly attributable to long-term memory encoding processes that are associated with the interaction between the amygdala and hippocampal regions. In contrast, the recency effect has typically been described as reflective of working memory processes, perhaps involving "anterior" circuits (Tops et al. 2004). 
Thus, the presence of anxiety may also interfere with the acquisition of negative information (i.e., within the recency position) due to disruption of anterior circuits. To date, the hypothesized mechanisms and relationships have not been adequately explored, and they are targets for future studies within the laboratory.

Of note, the effects of anxiety on affective learning have only been observed for the initial learning trials of the AAVL. This suggests that these disruptive effects are only temporary, as anxious individual perform normally with no discernible alterations in the learning of affective words in subsequent trials.

\section{Important Scientific Research and Open Questions}

Questions have been raised regarding the influence of anxiety on affective learning. Of importance is the poorly understood relationship between cortical and subcortical structures, autonomic regulation, affective learning, and anxiety. Given technological limitations, the studies using the AAVL have not adequately explored such relationships. However, the availability of high-resolution imaging technology will likely serve to advance conceptualization and understanding of the neuronal mechanisms that are associated with such processes. To date, no attempts have been made to control for the use of differing encoding strategies between high and low anxiety groups. In addition, to date the presence of potentially meaningful gender differences, particularly with regard to differences in regional brain activity has not been adequately examined as it relates to anxiety and affective learning. It is well known that such differences exist for other channels of emotion processing such as facial affect and emotional prosody. Thus, it is reasonable to speculate that gender differences would exist for affective word learning, although data from a representative sample has yet to be collected. Finally, affective learning among clinical populations (i.e., individuals formally diagnosed with anxiety disorder) has not been examined to date. It is speculated that more dramatic effects on affective learning would be observed for these individuals.

\section{Cross-References}

- Affective and Emotional Dispositions for Learning

- Affective Dimensions of Learning

- Stress and Learning

- Stress Management

\section{References}

Black, S., Everhart, D. E., Durham, T. W., Walker, M., Golden, J., \& Demaree, H. A. (2008). The effects of anxiety on affective learning and serial position recall. International Journal of Neuroscience, 118, 1269-1285.

Everhart, D. E., Demaree, H. A., \& Harrison, D. W. (2005). The merging of cognitive and affective neuroscience: Studies of the affective auditory verbal learning test. In F. Columbus (Ed.), Causes, role, and influence of mood states. Haupaugge: Nova Science.

Foa, E. B., \& Kozak, M. J. (1986). Emotional processing of fear: Exposure to corrective information. Psychological Bulletin, 99, 20-35.

Snyder, K. A., \& Harrison, D. W. (1997). The affective auditory verbal learning test. Archives of Clinical Neuropsychology, 12, 477-482.

Toglia, M. P., \& Battig, W. F. (1978). Handbook of word norms. Hillsdale: Lawrence Erlbaum.

Tops, M., van der Pompe, G., Wijers, A. A., Den Boer, J. A., Meijman, T. F., \& Korf, J. (2004). Free recall of pleasant words from recency positions is especially sensitive to acute administration of cortisol. Psychoneuroendocrinology, 29, 327-338.

\section{Effects of Exercising During Learning}

\section{MaRen SCHMidT-Kassow}

Institute of Medical Psychology, Frankfurt am Main, Germany

\section{Synonyms}

Ergometer cycling; Physical activity; Sport; Treadmill running; Verbal learning; Vocabulary acquisition

\section{Definition}

Exercising during learning: physical activity such as ergometer cycling or treadmill running that is executed simultaneously to a learning task (e.g., vocabulary learning).

\section{Theoretical Background}

These days, it is uncontroversial that regular exercise promotes not only cardiovascular but also cognitive health. Hence, Marcus Tullius Cicero's (106-43 B.C.) statement that it is exercise alone that supports the spirits, and keeps the mind in vigor is still totally upto-date. A rapidly growing field of research focuses on the neurobiology of exercise or influence of exercise on 
cognition, respectively. In this context, basically three hypotheses on how physical activity may have an effect on cognitive functions including learning have been formulated: First, the oxygen saturation hypothesis supposes that exercise increases the rate of oxygen consumption which in turn results in faster reaction times and better performance (Kramer et al. 1999).

Second, the neurotransmitter hypothesis states that increased releases of certain neurotransmitters as induced by physical activity, modulate information processing, hence resulting in faster information transfer (McMorris et al. 2008).

Third, there is cumulating evidence on a biochemical level that physical exercise leads to an increased release of neurotrophic factors (Schinder and Poo 2000), which in turn should mediate the effects of exercise and cognition. According to this neurotrophin hypothesis, neurotrophins support neuronal growth and differentiation and should hence be responsible for the positive effect on cognition. Indeed, multiple animal studies have shown a strong influence of physical activity on synaptic plasticity and, in particular, on the genesis of new neurons in the adult mammalian brain. However, until now, a conclusive statement on how exactly physical activity modulates human brain function is missing. In the last decade, a close relationship between physical activity and cognitive abilities has incrementally been confirmed for humans. Regular exercise has been shown to prevent cognitive decline in elderly which results in improved performance in reasoning tasks, working memory tasks, reaction time, or vocabulary measurements in physically active elderly compared to same age non-active participants. The same turns out to be true for young adults and children as has been shown by a positive correlation of exercise, learning, and intelligence in this age groups. Neuropsychological studies provided further evidence that physical activity positively influences cognition as shown in functional magnetic resonance imaging (MRI) and event-related potentials (ERP) studies.

\section{Important Scientific Research and Open Questions}

In line with the neurotrophin hypothesis, it has been shown (Winter et al. 2007) that performance in verbal learning tasks is enhanced if subjects showed an increased release of a the brain-derived neurotrophic factor (BDNF). This increased release of BDNF is induced by physical activity, i.e., treadmill running, prior to vocabulary learning. Hence, there is human evidence in favor of the neurotrophin hypothesis supporting the high relevance of neurotrophins in the context of learning. Further evidence for a close relationship between physical activity and learning comes from neuropsychological tests showing that simultaneous physical activity at a moderate intensity level also positively influences learning mechanisms (Schmidt-Kassow et al. 2010). Hence, moderate simultaneous ergometer cycling during auditory vocabulary learning improves performance in vocabulary tests compared to learning in physically passive situation. Furthermore, simultaneous cycling results in neuronal plasticity changes as evidenced by changes in the N400 amplitude. The N400 is an event-related potential as measured by an electroencephalogram (EEG) that is sensitive to learning-induced changes in cortical plasticity and proficiency in a second language. After participating in a vocabulary learning experiment, simultaneously physically active participants show a larger N400 effect compared to physically inactive participants. Given the fact that people who simultaneously cycle perform better in vocabulary tests and show a larger N400 effect, physical activity does not only enhance cognitive functions in general, but also pushes the establishment of new memory traces.

However, until now, modulating biochemical factors which drive improved performance in simultaneous exercise settings are subject to further research. In this context, the brain-derived neurotrophic factor (BDNF) has been extensively discussed as it induces changes in cortical plasticity and is involved in mnemonic processes. Future studies have to follow this issue by tracking participants' BDNF level serum while they are learning and exercising.

Other interesting issues concern the relevance of exercise intensity on the learning progress, i.e., is it sufficient to just move (e.g., walking), or is a certain intensity level indispensable to get the desired positive effect? Pre-activation of the leg motor cortex (standing compared to sitting) results in significantly improved performance in a picture-naming task in patients with chronic aphasia (Meinzer et al. 2011). Hence, even minimal movement patterns seem to positively influence linguistic processes, at least in patient populations. Thus, the question whether low-intensity walking is sufficient to enhance learning skills is 
particularly relevant with respect to aging subjects or patients with various diseases who are restricted in terms of their physical capacity.

\section{Cross-References}

- Memory Codes (and Neural Plasticity in Learning)

- Neuropsychology of Learning

- Vocabulary Learning in a Second Language

- Word Learning

\section{References}

Kramer, A. F., Hahn, S., Cohen, N. J., Banich, M. T., McAuley, E., Harrison, C. R., et al. (1999). Ageing, fitness and neurocognitive function. Nature, 400, 418-419.

McMorris, T., Collard, K., Corbett, J., Dicks, M., \& Swain, J. P. (2008). A test of the catecholamines hypothesis for an acute exercisecognition interaction. Pharmacology and Biochemistry of Behavior, 89, 106-115.

Meinzer, M., Breitenstein, C., Westerhoff, U., Sommer, J., Rösser, N., Rodriguez, A. D., Harnish, S., Knecht, S., \& Flöel, A. (2011). Motor cortex preactivation by standing facilitates word retrieval in aphasia. Neurorehabilitation and Neural Repair, 25, 178-187.

Schmidt-Kassow, M., Kulka, A., Gunther, T. C., Rothermich, K., \& Kotz, S. A. (2010). Exercising during learning improves vocabulary acquisition: Behavioral and ERP evidence. Neuroscience Letters, 482, 40-44.

Schinder, A. F., \& Poo, M. (2000). The neurotrophin hypothesis for synaptic plasticity. Trends in Neuroscience, 23, 639-645.

Winter, B., Breitenstein, C., Mooren, F. C., Voelker, K., Fobker, M., Lechtermann, A., Krueger, K., Fromme, A., Korsukewitz, C., Floel, A., \& Knecht, S. (2007). High impact running improves learning. Neurobiology of Learning and Memory, 87, 597-609.

\section{Effects of Multimedia Redundancy in History Learning}

\author{
SiI Ching Hit, Soon Fook Fong
}

School of Educational Studies, University Sains

Malaysia, Gelugor, Penang, Malaysia

\section{Synonyms}

Cognitive overload; Multimedia modality

\section{Definition}

Multimedia redundancy refers to a simultaneous presentation of different multimedia modalities that include text, graphics, and still images with redundant video, sound, and animation. The use of multimedia redundancy presentation in the history classroom involves simultaneous presentations of stimuli through different sensory channels which will provide additional stimuli reinforcement. In other words, multimedia redundancy program provides the students to use all their senses to explore the presentation. It has been argued that the combination of redundant channels of information will encourage learners to engage in active learning by representing the materials to be learned in words and in pictures and by generating connections between the pictorial and verbal representations. By combining multimedia technology and its variety of tools with the possibilities for individualized instruction and the interactive component, history topics will come alive for students, offering new light to facts with photographs, video, film, primary source documents, maps and music, making learning an active process and thus enhancing and facilitating their learning in history.

\section{Theoretical Background}

The use of multimedia presentation in history can be an essential means of recreating the past - making historical information alive, the intangible tangible, the past present, and bringing the excitement and relevance of historical study to students (Bulter and Clouse 1994). In this context, historians note that multimedia facilitate the development and presentations of census material, historical simulations, and supplement instructional resources. Easley (1998) reported that only $7 \%$ of successful communication between teachers and students comes from the spoken word, but $55 \%$ came from visual elements. This is especially valuable for history learning since the nature of the subject and the type of materials used to convey ideas and information are very visual. Moreover, the increasingly visual domination of mass communication now practiced in the world makes it both natural and essential for teachers to incorporate the evaluation of visual sources into history classroom.

Accordingly, students' learning in history is believed to be successfully met when using a multimedia redundancy presentation which refers to the integration of media to support and facilitate cognitive processes grounded on Mayer's (2001) Cognitive Theory of Multimedia Learning and Sweller's 
Cognitive Load Theory (2005). Mayer's Cognitive Theory of Multimedia Learning ascertains that learners are much more likely to learn effectively if information is presented both verbally and visually simultaneously. On the other hand, the necessity of adapting instructions to the constraints of the learner's cognitive system has been the main concern of Cognitive Load Theory (CTL). In order to stimulate effective learning, efficient instructional designs should be able to reduce extraneous cognitive load and at the same time increase germane cognitive load.

According to Mayer (2001), multimedia redundancy increases cognitive load, decreases working memory capacity, and reduces information processing capability, thus affecting learning. However, recent studies by Schnotz and Kürschner (2007) proposed a new perspective on CLT by suggesting that reduction of cognitive load is not always helpful for learning; learning could be improved by an increase of germane load by integrating multiple sources of information in order to create an adequate alignment of learner expertise and learning task difficulty. Germane cognitive load would occur when specific cognitive activities are performed in addition to the ordinary performance of a learning task and aiming at the further improvement of learning. However, whether the instructional materials impose extraneous cognitive load or germane cognitive load is not only affected by instructional design but is mediated by the learners' learning activities, which in turn depend on the motivational aspects personal goals and interests of the learner.

Recent studies by Seufert, Schütze, and Brünken (2009) indicate that multiple information could be comprehended by high-capacity learners who are more capable to retrieving, storing, and processing all the information at the same time in their working memory. Furthermore, the requirements of learning with multiple learning materials could enhance learning depending on learner's memory strategy skills and working memory capacity. For learning to occur, learners must actively engage in processes that impose a germane cognitive load which is facilitated by the instructional design. These findings support the addictive effect of multimedia redundancy through multiple-channel presentations. The combinations of redundant channels of information will encourage learners to engage in active learning by mentally representing the materials in words and in pictures and by mentally making connections between the pictorial and verbal representations. Both visual and auditory stimuli impact learner attention and this will eventually result in increased learning and retention.

\section{Important Scientific Research and Open Questions}

Multimedia redundancy can increase cognitive load, decrease working memory capacity, and reduce information processing capability, thus affecting learning. How could we account for the results of some studies that found multimedia redundancy improving history learning? Multimedia redundancy can be a valuable learning experience when students are engaged through interaction and receive adequate instructional preparation. Multimedia redundancy has the power to deliver high-quality and consistent multidimensional messages to the students. The use of multimedia-based instructional programs appears to motivate students, it has the potential to augment and extend the capabilities of good instruction in history classroom by motivating students through enriching history-learning situations that create rich-information environment, assist the students to experience the relevance and realistic scenarios, thus facilitating their learning in history.

This indicated that the multimedia lesson has a strong motivational function on students' learning. Students would be interested and excited about using a multimedia computer-based learning in a history lesson; the value of motivation was evident here to increase students' task perseverance, willingness to seek additional related content, and inclinations to process lesson content deeply. Thus, it was possible that the multimedia redundancy lesson did provide the cue for gaining and maintaining students' attention, increase relevance of the lesson for them, enhance their confidence of success in the learning task, and augmenting their satisfaction with the learning experience.

\section{Cross-References}

- Audio-Video Redundancy

- Cognitive Load Theory

- Multimedia Learning

- Redundancy Effect 


\section{References}

Bulter, J. D., \& Clouse, R. W. (1994). Educational technology and the teaching of history: Promise. practice, and possibilities. ERIC Document Reproduction Service No. ED 373005.

Easley, L. J. (1998). The enhanced lecture: A bridge to interactive teaching. In D. A. Trinkle (Ed.), Writing, teaching, and research history in the electronic age (pp. 65-72). New York: M.E. Sharpe.

Mayer, R. E. (2001). Multimedia learning. Cambridge: Cambridge University Press.

Schnotz, W., \& Kürschner, C. (2007). A reconsideration of cognitive load theory. Educational Psychology Review, 19, 469-508.

Seufert, T., Schütze, M., \& Brünken, R. (2009). Memory characteristics and modality in multimedia learning: An aptitude - treatment - interaction study. Learning and instruction, 19, 28-42.

Sweller, J. (2005). The redundancy principle in multimedia learning. In R. Mayer (Ed.), The Cambridge handbook of multimedia learning (pp. 159-168). New York: Cambridge University Press.

\section{Effects of Physical Context Change and Perceptual Learning on Generalization}

\section{James B. Nelson}

University of the Basque Country, San Sebastian, Gipuzkoa, Spain

\section{Synonyms}

Contextual control; Enhanced discrimination; Flattening generalization gradients

\section{Definition}

The effects of context and perceptual learning on generalization refers to the outcomes produced by changes in the background in which stimuli are encountered (context), and processes that affect the way stimuli are perceived (perceptual learning), on the extent to which stimuli are perceived as similar (generalization).

\section{Theoretical Background}

Generalization refers to the extent to which stimuli are perceived as similar, and is not a static phenomenon. Rather, humans' and animals' perception of, and generalization between, stimuli are affected by their experience with those stimuli. Indeed, early literature can be found that debated whether anything but complete generalization could be observed without prior experience with the stimuli in question (see Honig and Urcuioli 1981, for review).

The effect of experiences that alter perception, and hence generalization, are commonly thought to be the result of some type of perceptual learning. It is an important phenomenon, especially in the development of fine discriminations such as those demonstrated in experts (e.g., wine tasters). Theoretically, perceptual learning has been characterized in conflicting ways as both an associative-learning phenomenon and a nonassociative process. Nevertheless, consensus among the varying viewpoints can perhaps be reached with one adapted from those discussed in Nelson (2009). A common theoretical assumption regarding these phenomena is that stimuli are composed of multiple elements or components and generalization is a function of the extent to which components are shared between stimuli. From that assumption, perceptual learning can be viewed as a learning process (or processes) that improve(s) the perception of those elements that are unique to the stimulus; those that allow the stimulus to be differentiated from others (the collection found in Nelson 2009, review these issues). Perceptual learning thus reduces generalization, linking the two phenomena such that one cannot be effectively discussed without consideration of the other.

Context has long been known to be important for both learning and the expression of learning. Often, testing must be conducted in the same context where learning occurred for that learning to be fully retrieved and affect behavior. The context becomes especially important when the stimuli being learned about are associated with many different things. For example, humans may learn multiple lists of words for subsequent retrieval, and their ability to remember one list will depend on the context more than if they had only learned a single list. A rat might learn that a tone predicts a food pellet, and later learn that the same tone predicts a shock. In this case, where the tone is associated with different stimuli, learning about the tone and the shock will be more dependent on the context for retrieval than if the tone had not first been associated with food (see Bouton 2004, for review).

The effect of perceptual learning on generalization is to reduce generalization. A response learned to one stimulus generalizes less to another after manipulations (such as simple exposure to the stimuli) thought to induce perceptual learning. Interestingly, this learning 
phenomenon itself does not generalize well to other stimuli. To illustrate, performance in a visual bisection task, where participants are presented with three small lines and must determine whether the center line is closer to the left or right line, once mastered, is disrupted if the position of the lines on the computer screen is changed. A change in the position of the stimuli relative to the background contextual stimuli provided by the computer screen disrupted perceptual learning (Crist, Kapadia, Westheimer, and Gilbert, 1997 - referenced in Nelson and Sanjuan 2008). Thus, generalization, perceptual learning, and context are interrelated. The effects of perceptual learning on generalization cannot be fully understood without also considering the effect of context on perceptual learning itself.

As learning processes, aspects of perceptual learning and generalization should be sensitive to context changes. This prediction comes to the fore from associative theories of perceptual learning (e.g., McLaren and Mackintosh, 2000, - referenced in Nelson and Sanjuan 2008). In these theories, perceptual learning results from the extent to which the components of a stimulus are retrieved or activated by way of the associations among the various components themselves and associations with other aspects of the experimental situation. Some subset of these associative-learning processes can reasonably be assumed to be context dependent.

\section{Important Scientific Research and Open Questions}

The effects of physical context changes on perceptual learning and generalization appear to be both an attenuation of perceptual learning and an increase in generalization. Nelson and Sanjuan (2008) provide one illustration of these effects in humans. Participants played a video game where they were clicking a computer mouse to fire missiles at a spaceship. During a conditioning phase, a multicolored sensor (e.g., predominately green with a small stripe of blue on the edge) appeared that predicted an attack from the spaceship, and that participants should suppress their own firing in preparation for that attack. Testing followed conditioning, where a similarly colored sensor (e.g., predominately green with a small stripe of yellow on the edge) appeared. Participants that were simply conditioned and tested behaved to the test stimulus in almost exactly the same way as they behaved to the stimulus that was used in training. They suppressed their mouse clicking to both stimuli. That is, strong generalization was observed. Other participants had been exposed to the training and test stimuli prior to the conditioning phase. That exposure provided the opportunity for perceptual learning to take place so that they could better recognize the differences between the sensors. These latter participants showed little suppression of their behavior in the presence of the test sensor. The perceptual learning that occurred prior to conditioning reduced generalization between the two similar stimuli on test.

Two other groups were treated identically to those just described, except that they received a context change before the test. As part of the game, they were informed that they were being transported to a different galaxy for further testing and the star-field background upon which the gameplay took place was changed. On test, these two groups that received a change in the context both showed strong behavioral suppression. That is, they both showed robust generalization between the training and test stimuli. That strong generalization was the case regardless of whether or not conditioning had been preceded by perceptual learning. The effect of perceptual learning was attenuated with a change in the context in which it occurred.

As stated earlier, groups that had not received exposure to the stimuli prior to conditioning showed strong generalization regardless of the test context. To add to that, responding to the training stimulus was somewhat reduced with a context change. Thus, if responding to the similar test stimulus was expressed as a percentage of responding to the training stimulus, responding to the test stimuli would show as a higher percentage when testing was in a different context. Expressed as a "relative gradient," generalization between the training and test stimulus increased somewhat with a context change, even in the absence of an explicit perceptual learning manipulation. These results conceptually are the same as those found by Thomas and McKelvie (1982) who showed, in a completely within-subjects design with pigeons (Thomas and McKelvie 1982, Experiment 2), that generalization of responding to a particularly colored disk also increased with a context change when responding to the test stimulus was expressed relative to the training stimulus. 
The results of Nelson and Sanjuan (2008) showed that perceptual learning reduced generalization. That result is consistent with a large literature of findings (further examples are discussed in the works found in Nelson 2009). Nelson and Sanjuan's findings also demonstrate that generalization is increased, and perceptual learning is decreased, with a change in context. Other results from perceptual-learning tasks with both humans and animals are consistent with the observation that perceptual learning is at least partially contextually controlled. In addition to the visual bisection task mentioned earlier, subtle discriminations acquired over trials between two differently oriented distributions of visual noise are disrupted with changes in the background stimuli upon which those distributions are presented (Petrov, Dosher, and Lu, 2006 - referenced in Nelson and Sanjuan 2008). In these examples, fine discriminations are mastered, and then disrupted with a change in either the background contextual stimulation or the position of the stimuli with respect to the background. Rats that have been preexposed to different floor textures in a maze are better able to discriminate those textures when required to choose one arm over another for reward. That enhanced ability to discriminate was attenuated when the maze was located in a different environment than where the preexposure occurred (Trobalon, Sansa, Chamizo, and Mackintosh, 1991 - referenced in Nelson and Sanjuan 2008).

The passage of time has been convincingly argued to represent a functional change in background context (see Bouton 2004). Accordingly, the passage of time between training and testing has been shown to increase generalization in many cases, though the effect is slight (see Bouton et al. 1999, for review). Most often, what occurs in these tests is what was observed by Thomas and McKelvie (1982) with a physical context change: Responding to the cue that was initially trained is reduced more than responding to the similar test cues. Thus, as a percentage of the responding elicited by the initially trained stimulus, generalization appears to have increased.

Perceptual learning reduces generalization by enhancing the ability of stimuli to be discriminated from each other. Context operates on generalization by increasing the extent to which different stimuli are treated similarly. A part of that increase may be due to the operation of perceptual learning mechanisms being somewhat context specific.

Not all aspects of perceptual learning, however, are necessarily context dependent. For example, in the original demonstration of perceptual learning in animals (Gibson and Walk, 1956 - referenced in $>$ Animal Perceptual Learning, this volume), rats were exposed to the relevant stimuli in their home cages and tested elsewhere. Yet, the rats still showed a robust ability to discriminate. Other work has indicated that in some complex situations, perceptual learning may be more apparent, as opposed to less apparent, with a context change (see Channell and Hall, 1981 - referenced in Nelson and Sanjuan 2008). It is important to appreciate that as the complexity of the stimuli in an experiment increases, there are many mechanisms upon which a context change might operate. Mechanisms that may mask perceptual learning in one context may be lost with a change in context, revealing the effect of perceptual learning even though that learning itself may be somewhat attenuated with the context change. At the theoretical level, the exact processes responsible for behavior indicative of perceptual learning and generalization upon which the context change operates have yet to be determined.

\section{Cross-References}

- Animal Perceptual Learning

- Generalization Versus Discrimination in Learning

- Perceptual Learning

\section{References}

Bouton, M. E. (2004). Context and behavioral processes in extinction. Learning \& Memory, 11, 485-494.

Bouton, M. E., Nelson, J. B., \& Rosas, J. M. (1999). Stimulus generalization, context change, and forgetting. Psychological Bulletin, $125,171-186$

Honig, W. K., \& Urcuioli, P. J. (1981). The legacy of Guttman and Kalish (1956): 25 years of research on stimulus generalization. Journal of the Experimental Analysis of Behavior, 36, 405-445.

Nelson, J. B. (Ed.). (2009). Perceptions of perceptual learning [Special section]. Learning \& Behavior, 37, 117-160.

Nelson, J. B., \& Sanjuan, M. C. (2008). Flattening generalization gradients, context, and perceptual learning. Learning \& Behavior, 36, 279-289.

Thomas, D. R., \& McKelvie, A. R. (1982). Retrieval of memory in the pigeon by context manipulations. Animal Learning \& Behavior, 10, 1-6. 


\section{Effects of Remedial Mathematics on the Learning of Economics}

\author{
Johan N. M. Lagerlöf ${ }^{1}$, Andrew J. Seltzer ${ }^{2}$ \\ ${ }^{1}$ Department of Economics, University of Copenhagen, \\ København K, Denmark \\ ${ }^{2}$ Department of Economics, Royal Holloway, \\ University of London, Egham, UK
}

\section{Synonyms}

Tending to remedy math abilities

\section{Definition}

Remedial mathematics is defined as the intention to correct or improve one's skill in mathematics. In this entry, a particular focus is on learning economics at university level. Actually, university-level economics presupposes an extensive use of mathematics which results in difficulties to learn economics. Consequently, many universities offer remedial math courses to students in order to compensate their difficulties to learn economics.

\section{Theoretical Background}

University-level economics makes extensive use of basic mathematics, which makes the subject difficult for less technically able students. Indeed, a number of studies have shown that there is a positive correlation between students' mathematics background and their performance in economics degrees (e.g., Anderson et al. 1994; Butler et al. 1994). In an attempt to tackle the problem with underprepared students, many universities offer remedial math courses to students early on in their university studies. This practice has sometimes, in particular in the USA, been criticized, on the grounds that tax money should not be used in colleges to teach high school courses. Supporters of remedial education, however, have argued that it aids less technically able students, who are often from disadvantaged backgrounds, and facilitates their integration into university studies.

When assessing the possible virtues of remedial mathematics courses, a key question is whether they actually work. That is, does taking remedial math improve students' performance in their economics courses relative to how they would have done without it? To empirically investigate that question is associated with difficult methodological problems. A naive approach would be to simply compare the performances (e.g., exam results) of a group of students who have taken a remedial course with those of another group who have not. However, students who take remedial mathematics are likely to be systematically different from those who do not, and these selection issues are likely to lead to either under- or overestimate of the true effect of remediation. For example, if students are assigned to a remedial course based on their secondary school curriculum, as is a common practice, the difference in average performance across the groups is likely to reflect differences in secondary education as well as the effects of remediation. This means that the comparison would yield an estimate of the true remediation effect that is biased downward, as the students assigned to the remedial course in the typical case would have a weaker math background than the others. Alternatively, if students were not assigned to the remedial course but instead had a free choice whether to take it, then estimated effects are likely to be biased upward as students who are more motivated might be more likely to self-select into the remediation group. Another possibility is that the nonremedial students chose not to take the course because they knew that they were relatively strong in math and therefore did not need remediation, leading (again) to an underestimation of the true effect. Notice that the process of selection is likely to be driven by factors that are unobservable to the researcher, and thus the standard approach of simply adding regression controls is unlikely to eliminate all sources of omitted variable bias.

Ideally, to deal with these methodological issues, one would like to be able to run a controlled experiment in which a group of students is randomly allocated into a compulsory remedial course, while a similar group is not allowed to take the course. Of course, such an experiment would in many cases be impractical and it might also raise ethical concerns. Nevertheless, there is a small literature that has been able to use experimental or quasi-experimental methods (see Angrist and Pischke 2008, for a useful discussion of quasi-experimental methods) to examine 
the effects of random differences in the probability of being assigned to study remedial mathematics on student performance in economics courses. Below we will briefly discuss three of these studies: Pozo and Stull (2006), Bettinger and Long (2009), and Lagerlöf and Seltzer (2009). As far as we are aware, these three studies are the only ones in the literature that convincingly, or reasonably convincingly, identify the causal effect of taking a remedial math course on the learning of economics.

\section{Important Scientific Research and Open Questions}

The study by Pozo and Stull (2006) examines a controlled experiment in a Principles of Macroeconomics course at Western Michigan University. Students taking the course had access to an online math tutorial and test, but some of them (randomly selected) were given a small grade incentive to actually do the tutorial and perform well in the test. Pozo and Stull find that the grade incentive only had short-term effects. It had a positive and statistically significant impact on the grade for the course's midterm exam, taken shortly after the tutorial, but a smaller and statistically insignificant effect on the grade for the final exam at the end of the semester.

Bettinger and Long (2009) examine the effects of remedial courses in math in the Ohio public university system. In order to control for possible selection problems, Battinger and Long use the estimated probability of receiving remediation as an instrument for having actually received remedial education. They find that remediation had a positive and statistically significant effect on several long-term performance measures. Students with a higher probability of being exposed to remediation were, other things being equal, less likely to drop out of college and more likely to transfer to a higher-level college and to complete a bachelor's degree. However, Bettinger and Long's methodological approach raises two questions about the interpretation of their findings. First, their measure of remediation is based on school-level differences in remedial offerings, which could be correlated with other aspects of the schools' focus on undergraduate education. Second, they find that students with a high probability of being assigned to remedial math were less likely to do their degree in a mathematically oriented subject than similar-ability students with a low probability of assignment. This suggests that remediation might have a limited effect in increasing students' mathematical abilities, but helps to match students with appropriate degree subjects.

Lagerlöf and Seltzer (2009) make use of a natural experiment created by the implementation of a remedial mathematics course by the Economics Department of Royal Holloway, University of London. Prior to 1999 the department did not offer remedial math to any students, but in 1999 a remedial mathematics course lasting 7 weeks became compulsory for students who had not studied mathematics beyond age 16 or had performed poorly in secondary school mathematics. Importantly, the remedial course was not made available to other students. Lagerlöf and Seltzer compare the performance of the cohorts admitted prior to 1999 to that of the 1999 cohort in a variety of compulsory economics courses using a differencein-difference approach. The effects of remedial mathematics are identified by comparing the change in performance of the math-deficient students (who were required to take remedial courses after 1999) to the change in performance of students with a stronger math background (who did not take the remedial course prior or subsequent to 1999). Lagerlöf and Seltzer find no evidence that remedial math has an effect on students' performance in Principles of Economics or Quantitative Methods, the two core courses in the first year of the economics degree. Moreover, they find no evidence of longer-term effects, as performance in the second and third year of the program is unrelated to exposure to the remedial course. Surprisingly, the only statistically significant results that they find for the effectiveness of remedial mathematics are for Economics Workshop, a nonmathematical first-year course assessed by two essays, and for the overall average across first-year subjects.

In summary, Pozo and Stull (2006) provide convincing evidence that access to a remedial mathematics program had a positive short-term effect, but they do not find much evidence that the effect lasted even to the end of a one-semester course. Bettinger and Long (2009) find that increased access to remediation improved a number of short-term and long-term 
outcomes, although their results may simply reflect the fact that students with access to remediation may have also had access to other resources or that remediation only helped students by matching them to appropriate degree subjects, rather than improving their math skills. The results of Lagerlöf and Seltzer (2009) are perhaps more pessimistic still. They find that exposure to a fairly extensive remedial course did not have a statistically significant effect on student performance in any mathematically oriented university-level economics subject.

Overall, it seems fair to conclude that there is currently no evidence that remedial mathematics actually has an effect on performance in university-level economics courses that is (i) long-term and which (ii) can be conclusively attributed to remediation bringing about improved math skills.

\section{Cross-References}

- Abstraction in Mathematics Learning

- Design Experiments

- Experimental and Quasi-experimental Designs for Research on Learning

- Incentives and Student Learning

- Mathematical Learning

- Remedial Learning

\section{References}

Anderson, G., Benjamin, D., \& Fuss, M. A. (1994). The determinants of success in university introductory economics courses. Journal of Economic Education, 25, 99-119.

Angrist, J. D., \& Jörn-Steffen Pischke, J.-S. (2008). Mostly harmless econometrics: An empiricist's companion. Princeton and Oxford: Princeton University Press.

Bettinger, E. P., \& Long, B. T. (2009). Addressing the needs for under-prepared students in higher education: does college remediation work? The Journal of Human Resources, 44, 736-771.

Butler, J. S., Finegan, T. A., \& Siegfried, J. J. (1994). Does more calculus improve student learning in intermediate micro and macro economics theory? American Economic Review (Papers and Proceedings), 84, 206-210.

Lagerlöf, J. N. M., \& Seltzer, A. J. (2009). The effects of remedial mathematics on the learning of economics: Evidence from a natural experiment. Journal of Economic Education, 40, $115-137$.

Pozo, S., \& Stull, C. A. (2006). Requiring a math skills unit: results of a randomized experiment. American Economic Review (Papers and Proceedings), 96, 437-441.

\section{Effects of Task Difficulty in Listening Comprehension}

\author{
Nooreen Noordin ${ }^{1}$, Katrin Shamshiri $^{1}$, Azahari Ismail ${ }^{2}$ \\ ${ }^{1}$ Department of Language Education and Humanities, \\ Universiti Putra Malaysia, Serdang, Selangor, Malaysia \\ ${ }^{2}$ Department of Professional Development and \\ Continuing Education, Universiti Putra Malaysia, \\ Serdang, Selangor, Malaysia
}

\section{Synonyms}

Difficulty level of authentic listening input; Listening task complexity

\section{Definition}

In order to learn about the effects of task difficulty in listening comprehension, it is necessary to define tasks and listening comprehension first.

Generally, task is a broad term which covers both real world (e.g., listening to the weather forecast) and pedagogical activities (e.g., a sentence completion listening task). With regard to language learning, only pedagogical tasks which include input and output are considered. A pedagogical task is defined as a "classroom work that involves learners in comprehending, manipulating, producing or interacting in the target language while their attention is focused on mobilizing their grammatical knowledge in order to express meaning" (Nunan 1999, p. 25).

A brief definition of listening comprehension is the ability to recall and understand information which is presented orally. What makes a listening task difficult is its ongoing process that requires foreign/second language learners to comprehend and listen to simultaneously which may even cause anxiety (Kao 2006).

Considering the definitions above, listening task difficulty can be defined as a way that can/may determine students' success or failure in completing a listening task.

\section{Theoretical Background}

Investigating the effect of task difficulty in listening comprehension encompasses various research disciplines, such as processing capacity and working 
memory, communicative process model, and code complexity. However, the theory underpinning task difficulty is cognitive load theory which addresses the limitations of working memory capacity and the construction of schema automation in long-term memory (Sweller 1988).

In listening, the listener usually has little control over the speech rate of the message which is moving along the auditory canal. In many cases, the listener does not have the chance to rewind what has been heard earlier. The listener has to listen, retain information first in the short-term memory which serves as a temporary storage for processing the received message, integrate it with incoming input, and adjust comprehension of what is heard with reference to the new information. All these steps impose a heavy cognitive load on working memory.

The basic criteria for organizing language learning tasks are cognitive load, communicative stress, particularity and genreralizability, code complexity and interpretive density, content continuity, and process continuity (Candlin 1987).

\section{Important Scientific Research and Open Questions}

The question which has been of increasing interest among researchers with regard to task difficulty and listening comprehension is that what factors contribute to the complexity of a task. The answer to this question cannot be confined only to a limited list of sources of difficulty. Among the major sources include the nature of the input, the response mode, the number of people involved in the task, the difficulty of the assessment criteria, the details of what is said, listening situation, and task type (one-way versus two-way).

The difficulty of the task and test items can also be attributed to pronunciation and accent of the speaker, rate of delivery, the effect of tape recorder, lack of vocabulary, lack of proficiency, lack of concentration, lack of practice, and other situational factors (Seferoglu and Uzakgoren 2004).

It is important to consider task type before exploring the sources of difficulty. In a multiple-choice listening task, for example, similarity of choices can confuse the learners which option to choose even if they have understood the content of the listening material. The number of words in each choice may also hinder listening comprehension. Students need to listen, read the choices, and decide which one to choose. Being engaged in listening and reading simultaneously can make a task very challenging.

In a note-completion task, on the other hand, students have to read and write as they listen, being aware of the fact that what they read does not exactly resemble what they hear in terms of wording and structure; instead, they need to comprehend the synonyms immediately and decide what words would better fill each blank. This is when they may face lack of time which in turn affects the results of their listening test. In other words, integration of several skills simultaneously affects listening comprehension.

In a table-completion task, the number of columns and rows, how fast the speakers moves from one cell to another, matching the summarized information in the table with the flow of lengthy speech, even lack of prior knowledge when the topic of discussion is culturebased (e.g., a special occasion in a country) or it is an introduction to a scientific invention or discovery, even a new product or sport, may account for task difficulty.

Researchers believe that strategy training can facilitate task difficulty to a great extent. Normally, students tend to be more reserved for the more difficult tasks; however, if they receive strategy training during the listening course before sitting the test, they are more likely to achieve better results. Language learning strategies include memory, cognitive, metacognitive, compensation, social, and affective strategies. These strategies help students to ask for clarification and repetition when they do not understand a piece of information on the listening track, while in the absence of strategy training, students either give up answering the questions or may complete the tasks individually without being engaged in group or pair-work. When the tasks are easy, students who have received strategy training show autonomy and complete the tasks more confidently.

Socio-affective strategy training has a facilitating role in completing a difficult task, while in the absence of strategy training, students fail to employ socioaffective strategies when faced with extremely difficult tasks. In other studies, it has also been postulated that listening comprehension improves students instructed in learning strategies on texts that are accessible, not on those that are too difficult and/or for which students lack relevant prior knowledge. In other words, students fail to employ listening strategies for unfamiliar and 
too difficult tasks (Chamot 2005, p. 117). Students' tendency to use fewer social and affective strategies may be the result of their proficiency level for that task which led them to automatically use the strategies without necessarily reporting them. In other words, once the strategies are internalized, students use them automatically and without hesitation.

Task difficulty and level of language proficiency have a major effect on the strategies that students use. For example, some strategies used by beginning level effective language learners are used less frequently by the same learners when they reach intermediate level classes, probably because they have to develop new strategies to meet the requirements of more challenging language tasks. In addition, the difficulty of the task seems to be related to whether students would even try to use learning strategies. If a task, for instance, is relatively easy, students can complete it as instantly as they would in their native language, without conscious attention to strategies. On the other hand, if the task is too difficult, even effective learning strategies cannot overcome the learner's lack of knowledge and/or language proficiency.

However, it should be mentioned that there are many other factors which may affect frequency use of strategies for easy or difficult listening tasks such as learner's style and preferences, motivation, cultural background, and students' attitude toward strategy use. Even the instrument which is used to measure strategy use can affect the results. The Likert scale, for example, may not always be an appropriate scale to arrive at precise and detailed data.

It is recommended that instructors assist learners to overcome unrealistic expectations about understanding everything they hear. By introducing the gist of the spoken language for the first time, students will gradually become comfortable with grasping the key words in a listening task.

\section{Cross-References}

Language (Discourse) Comprehension and
Understanding
- Metacognitive Strategies
Task Difficulty and Learning

\section{References}

Candlin, C. N. (1987). Towards task-based language learning. In C. N. Candlin \& D. F. Murphy (Eds.), Language learning tasks (pp. 5-22). London: Prentice Hall.
Chamot, A. U. (2005). Language learning strategy instruction: Current issues and research. Applied Linguistics, 25, 112-130.

Kao, C. C. (2006). EFL listening comprehension strategies used by students at the Southern Taiwan university of Technology. Unpublished doctoral dissertation, University of South Dakota, Vermillion.

Nunan, D. (1999). Second language teaching and learning. Boston: Heinle and Heinle.

Seferoglu, G., \& Uzakgoren, S. (2004). Equipping learners with listening strategies in English language classes. Hacettepe Universitesi Egitim Fakültesi Dergisi, 27, 223-231.

Sweller, J. (1988). Cognitive load during problem solving: Effects on learning. Cognitive Science, 12, 257-285.

\section{Effects of Testing on Learning}

\author{
Shana K. Carpenter
}

Department of Psychology, Iowa State University, Ames, IA, USA

\section{Synonyms}

Retrieval practice; Test-enhanced learning; Testing effect

\section{Definition}

Research on the effects of testing on learning has become increasingly popular over the last several years. The testing effect, as it is commonly called, refers to the learning advantages that occur as a result of trying to retrieve information from memory (i.e., being tested), in comparison to simply being reexposed to that information. For example, consider a student who is studying German and trying to learn new vocabulary by pairing a German word (Hund) with its English translation (Dog). The student could construct flashcards containing Hund on one side and Dog on the other. Each time she sees the German word, she must try to recall the correct English translation before turning over the card to check her answer. As an alternative strategy, she could construct cards containing both the German and English words on the same side (Hund - Dog), and try to commit these words to memory by reading them together over and over. According to research on this topic, the student will perform best on the upcoming vocabulary exam if she uses the former strategy rather than the latter. 


\section{Theoretical Background}

Why is it beneficial to test oneself over information? There are two primary means by which these benefits can occur - through indirect means and through direct means. Tests can benefit learning through indirect means by revealing to the learner which portions of the material are already well-learned (i.e., the information that was successfully retrieved on the test), and which portions are not yet well-learned (i.e., the information that could not be retrieved on the test). The learner can then use this information to guide subsequent study. For example, a student who is using flashcards to study German-English vocabulary may go through the stack of flashcards and try to recall the English translation to a German word. If the English translation can be readily recalled, the learner may decide to set this card aside and focus more time on the German words for which she cannot readily recall the English translations. Through the process of trying to retrieve an answer and then receiving feedback, therefore, tests may optimize the learning process by identifying which items require further study.

Interestingly, tests are known to benefit learning even when corrective feedback is not provided. This is generally true as long as the information is correctly retrieved on the test. Tests can therefore benefit learning through direct means - that is, through the act of retrieval itself. What is beneficial about the act of retrieval? There have been three general hypotheses to explain this. The first and earliest hypothesis was based on the total time hypothesis of learning (Cooper and Pantle 1967). This view proposes that, all else being equal, the more time a learner devotes to learning information, the better that information will be learned. Early research on the testing effect did not always control for the time that learners spent learning the material. In fact, many studies that reported beneficial effects of testing were based on a comparison of a learning method that utilized testing (e.g., the flashcard method described above) vs. no method at all. It was therefore unknown whether testing was beneficial because of the act of testing per se, or because it simply afforded the learner more time to spend learning the material.

The most common and straightforward way to test this hypothesis is to compare two learning methods: one that involves testing and another that provides an equivalent amount of exposure to the material but does not involve testing. Suppose learners are given the following list of words to memorize: Street, Flower, Horse, and Bridge. After seeing the list once, some learners are asked to recall all of the items from the list, whereas others are simply shown the word list again, exactly as they saw it the first time. All learners then complete a final test in which they are asked to recall the entire word list. Studies that have used this general method have falsified the total time hypothesis by demonstrating that even when the exposure time to the material is equated between the two methods, the testing method leads to better learning than does a simple reexposure method (e.g., Roediger and Karpicke 2006).

The second hypothesis to be advanced was based on the notion of transfer-appropriate processing (Morris et al. 1977). Simply stated, this view proposes that learning will benefit to the extent that the operations required by the final test match those that were required during initial learning. Learning information via testing could be more beneficial than through reexposure because the testing method provides practice at the same type of operations that will be required on the final test, whereas the reexposure method does not.

This hypothesis also lends itself to a fairly straightforward test. One could learn material using a testing method that is either similar to, or different from, the way in which that material will eventually be tested on the final test. For example, one could learn information from a textbook chapter by first trying to recall everything they can remember from that chapter (similar to an essay test). Later, the learner can complete a final test over that information that is either an essay test (similar to the initial testing method they used) or a multiple-choice test (different from the initial testing method they used). According to this view, information should be learned better if the final test is similar to the initial testing method. Research that has used this basic method has not confirmed this, however. Instead, it appears to suggest that initial testing methods that are more difficult (e.g., essay methods) lead to better learning, regardless of the way that information is tested on the final test (e.g., Kang et al. 2007).

This finding has led to another theoretical perspective that is based on elaborative processing. According to this hypothesis, testing is beneficial for learning because it stimulates the learner to think more deeply 
about the material and activate information that may facilitate its retrieval in the future. It is believed that this level of elaboration does not occur during a simple reexposure, because here learners are just viewing the material without actively engaging in the elaborative processing that is believed to occur during testing. Research has supported this theory by showing that initial test conditions that encourage elaborative processing by making information harder to retrieve (e.g., by using fewer cues, or by administering the test after a longer, as opposed to a shorter, time interval) can lead to better learning of that information (e.g., Carpenter 2009).

\section{Important Scientific Research and Open Questions}

Tests are widely used in educational settings as assessment devices. There appears to be less awareness among students and educators that tests can also be used as a means of promoting learning, however. Recent research has therefore attempted to increase the awareness of testing as a means of benefiting learning (not just assessing it), and to discover ways in which tests can be most effectively used as an educational tool.

Much of the research on the testing effect has been conducted in laboratory settings using relatively discrete stimuli such as lists of words or simple pictures. An important question, therefore, is whether the benefits of testing can apply to more complex learning materials in realistic educational environments. When high school students are studying for their exam on the French Revolution, for example, would they be better off to test themselves over the material than to review their notes? Recent research has made some progress in this area by demonstrating beneficial effects of testing on the learning of educationally relevant materials such as textbook chapters, general knowledge facts, foreign language vocabulary, and maps. Some studies have also been conducted in school settings to demonstrate that testing can benefit learning of information being taught in middle schools and high schools.

Another important theme in this research is how best to utilize testing in a way that will optimize learning over meaningful intervals of time. In addition to the flashcard method, how can learners make use of a testing strategy in order to improve their learning? How many times should they test themselves over information before it is fully learned? The widespread use of testing as an educational tool may not be advisable if its benefits are short-lived or limited to a specific set of materials. Therefore, another important question is how long these learning benefits last over time. After learning information through testing, how likely are students to remember that information after one day? One week? One year? Some progress has been made in these areas by demonstrating, for example, that learning benefits more when students retrieve something multiple times on a test, as opposed to just once, and that these benefits appear to persist over time intervals of several months.

Researchers have also begun to explore whether testing can promote the transfer of learning to information that was not directly tested. For example, if learners are able to recall that toucans are related to woodpeckers, does this help them to remember that toucans sleep in tree holes at night, even if the latter fact was never tested (e.g., see Chan et al. 2006)? Other questions that have emerged include whether there is a certain type of testing strategy that works best for promoting long-term retention and transfer, and whether testing benefits learning more than other types of organizational learning activities, such as outlining.

\section{Cross-References}

$\checkmark$ Cued Recall

- Elaboration Effects on Learning

- Elaboration Strategies and Human Resources Development

- Recall and Effect of Repetition on Recall

- Spread of Activation Theory

- Test-Enhanced Learning

\section{References}

Carpenter, S. K. (2009). Cue strength as a moderator of the testing effect: The benefits of elaborative retrieval. Journal of Experimental Psychology. Learning, Memory, and Cognition, 35, 1563-1568.

Chan, J. C. K., McDermott, K. B., \& Roediger, H. L., III. (2006). Retrieval-induced facilitation: Initially nontested material can benefit from prior testing of related material. Journal of Experimental Psychology. General, 135, 553-571.

Cooper, E. H., \& Pantle, A. J. (1967). The total time hypothesis in verbal learning. Psychological Bulletin, 68, 221-234.

Kang, S. H. K., McDermott, K. B., \& Roediger, H. L., III. (2007). Test format and corrective feedback modify the effect of testing on long-term retention. European Journal of Cognitive Psychology, 19, 528-558. 
Morris, C. D., Bransford, J. D., \& Franks, J. J. (1977). Levels of processing versus transfer appropriate processing. Journal of Verbal Learning and Verbal Behavior, 16, 519-533.

Roediger, H. L., III, \& Karpicke, J. D. (2006). The power of testing memory: Basic research and implications for educational practice. Perspectives on Psychological Science, 1, 181-210.

\section{Effects of Tracking and Ability Grouping on Learning}

\author{
Maureen T. Hallinan \\ Center for Research on Educational Opportunity, \\ University of Notre Dame, Notre Dame, IN, USA
}

\section{Synonyms}

Ability grouping; Tracking

\section{Definition}

A distinction must be made between $>$ tracking and - ability grouping, especially since the meaning of these terms has changed over the past several years. Initially, tracking referred to the assignment of students to one of usually three levels or programs of instruction: academic, general, or vocational. The academic program provided the most challenging curriculum and prepared students for admission to college. The general program included less-challenging courses and aimed to ready students for entrance to the labor market after high school. The vocational program taught students the skills specific to a particular trade, such as plumber, chef, auto mechanic, or seamstress.

In contrast, ability grouping referred to the assignment of students to instructional levels in particular courses within a classroom. Ability grouping typically was practiced for instruction in reading or language arts and mathematics. Within-class ability grouping generally characterized elementary school instruction. At the secondary level, ability grouping referred to curriculum levels across classes within grades. For example, students might be assigned an honors, regular, or basic level of instruction within the academic, general, or vocational track.

Over time, and in response to efforts to raise the achievement level of all students and to deal with budgetary shortfalls, schools discontinued vocational programs. Today, all students must take a number of required substantive courses supplemented by electives. Schools typically have three levels of academic difficulty: honors, regular, and basic. Small schools may have only one or two ability-group levels while larger, more heterogeneous schools could have four or more levels, such as advanced, honors, regular, basic, and very basic, although different names are usually given to the lower-level courses. Tracking, according to its earlier meaning of curricular differentiation within an entire school, no longer exists in most schools, while ability grouping now refers to the difficulty of the curriculum taught in different classes at a given grade level.

\section{Theoretical Background}

The aim of ability grouping is to enable a teacher to direct instruction to the cognitive abilities of the students being taught. Educators believe that presenting students with material that is too easy for them leads to boredom and disciplinary problems, while giving students a curriculum that is too difficult produces discouragement and disengagement from the learning process. Ability grouping is expected to avoid those two negative student outcomes by tailoring the curriculum and pedagogy to the students' cognitive capabilities.

A debate has arisen with respect to the effectiveness and fairness of ability grouping. Supporters of the practice argue that it makes instruction easier for teachers, enabling them to provide students with a curriculum that keeps them engaged and motivates them to produce their best effort to learn. Moreover, since the teachers of ability-grouped classes are aware of the cognitive abilities of their students, they are likely to have appropriate expectations for their students' performance. Advocates of ability grouping also argue that since the practice is basically a way of organizing students for instruction, it can be modified to improve the best fit between the structure of the curriculum and school and student characteristics. Therefore, ability grouping is amenable to efforts to reduce or eliminate any inequities associated with the practice. This can be accomplished by insuring that ability grouping reflects the theory that supports it.

Another way that schools can make ability grouping more effective and equitable is to maintain flexibility in ability-group assignments. If students are placed at an 
inappropriate level at the beginning of a semester, or experience faster or slower growth in achievement than their peers, they should be reassigned to a higher or lower group, regardless of the time during a school year the change is indicated. Ability-group assignments should also be independent of subject area, for example, a student may be assigned to a different abilitygroup level in reading or language arts than in mathematics.

Critics of ability grouping maintain that the practice has inherent inequalities that cannot be modified easily and that impede the goal of facilitating students' learning. While research shows no difference in the mean achievement of ability-grouped classes compared to heterogeneously or ungrouped classes, the practice does affect the distribution of students' achievement. Ability grouping favors high-achieving students by accelerating their rate of learning while disadvantaging low-ability students by slowing down their achievement gains. The reason for this outcome, critics claim, is that the quality of instruction varies across ability-group levels. Students in low-ability groups receive less coverage of the curriculum, and are taught by less-experienced teachers than those in higherability groups (Oakes 1985). In addition, teachers are likely to hold lower expectations for the performance of students in low-ability groups. Finally, many critics claim that ability grouping tends to have a segregating effect, with disproportionately more minority and disadvantaged students assigned to the lower-ability groups and fewer placed in the higher-ability groups.

\section{Important Scientific Research and Open Questions}

The vigorous ongoing debate about the advantages and disadvantages of ability grouping and the important policy implications of this practice has stimulated a large number of studies by education researchers and social scientists. The research focuses on the two main aspects of ability grouping: how students are assigned to ability groups and what are the effects of ability grouping on students' achievement.

Educators often consider both academic and nonacademic factors in determining a student's ability-group placement. The greatest amount of within-group homogeneity tends to occur when teachers rely primarily on standardized test scores to make ability-group assignments. Students are simply placed at a group level determined by their rank in class. This is more readily accomplished in mathematics and perhaps in science than in other subjects because evaluating mathematics skills is fairly straightforward. Other academic criteria may include a teacher's subjective decisions about a student's motivation and effort, student grades, teacher and counselor's recommendations, prior-group placement, and course prerequisites. Assignment to reading and language arts courses is more likely to include these additional assignment criteria. Nonacademic factors that influence placement decisions include course conflicts, cocurricular and extracurricular schedules, work demands, and teacher and curricular resources. For example, Barr and Dreeben (1983) show that the number of available text books and the size of a class determine the number of ability groups that are formed for reading instruction.

As a result of variation across schools, subjects, and teachers in the criteria used for placement decisions, the likelihood that a student is assigned to a specific group level is influenced by what school a student attends. Students with similar cognitive skills may be placed at different levels in different schools. School, class, and subject also influence the flexibility of ability-group assignments, with some schools and teachers making fewer adjustments in group assignments than others.

Research on the determinants of ability-group assignment also includes examination of the role that the socioeconomic status (SES) of students plays in the assignment process. Other studies reveal that race and ethnicity influence ability-group assignment. Minority and low-SES students are more likely to be placed in low-ability groups than are more advantaged students. This effect is reduced but not eliminated when academic achievement is controlled. The finding supports the contention of ability-group critics that abilitygroup assignments are biased against minority and disadvantaged students.

The second major area of research on ability grouping examines its effects on students' academic achievement. The main finding of these studies is that curriculum coverage and the quality of instruction vary by group level. In lower-ability classes, teachers tend to complete less of the curriculum than those in higher-ability classes. One reason is that teachers in lower-ability groups must devote more instructional time to student-discipline than is required in 
higher-level groups where students are more engaged with a challenging curriculum and skilled pedagogy. Consequently, students in low-ability groups learn less and have lower-achievement gains than those in higher-level groups. Studies also show that teachers of lower-ability groups tend to have weaker pedagogical skills than those in higher groups, resulting in less student-learning.

Research also demonstrates that ability grouping has no effect on the achievement outcomes of students in the middle-level ability groups. Its effects are only at the extremes of the achievement distribution. Apparently, instruction in the middle-ability groups is similar to that in schools with heterogeneous or no ability grouping. Interestingly, however, one study (Hallinan 2003) demonstrated that high-school students who were assigned to ability groups based on school or teacher criteria would attain higher achievement if they were placed in an ability group at the next highest level, and lower achievement if placed at the next lower level than the group level to which they were assigned originally. This finding holds regardless of a student's ability. Thus, the study suggests that, in general, highschool students are not being challenged sufficiently to attain their best performance.

While an extensive body of research has addressed issues regarding the effectiveness and fairness of the practice of ability grouping, questions remain (Carbonaro 2005; Gamoran 2000; and Walberg et al. 2006). Some social scientists and educators ask to what extent ability grouping and tracking can produce positive learning outcomes. Other scholars question the extent to which grouping disadvantages underprivileged students. These questions have to do with the pedagogical value of tracking and ability grouping and the segregative effects of the practice. The debate reveals ideological differences as well as differences in the interpretation of research findings (Carbonaro 2005; Gamoran 2000; and Walberg et al. 2006). What is needed to support enlightened policy governing ability grouping is research that broadens the scope of research to identify organizational and instructional processes that successfully raise the achievement of all students.

\section{Cross-References}

- Abilities to Learn: Cognitive Abilities

- Ability Grouping (and Effects) on Learning
Achievement Motivation and Learning

Composition of Learning Groups

\section{References}

Barr, R., \& Dreeben, R. (1983). How schools work. Chicago: University of Chicago Press.

Carbonaro, W. (2005). Tracking, student effort, and academic achievement. Sociology of Education, 78, 27-49.

Gamoran, A. (2000). Is ability grouping equitable? In R. Arum \& I. Beattie (Eds.), The structure of schooling: Readings in the sociology of education (pp. 234-240). Mountain View: Mayfield Publishing Company.

Hallinan, M. T. (2003). School organization and response to systemic breakdown. In M. T. Hallinan, A. Gamoran, W. Kubitschek, \& T. Loveless (Eds.), Stability and change in American education: Structure, process and outcomes (pp. 93-108). Clinton Corners: Eliot Werner Publications.

Oakes, J. (1985). Keeping track: How schools structure inequality. New Haven: Yale University Press.

Walberg, H. J., Reynolds, A. J., \& Wang, M. C. (Eds.). (2006). Can unlike students learn together?: Grade retention, tracking and grouping. Greenwich: Information Age Publishing.

\section{Effort}

- Measurement of Student Engagement in Learning

\section{Effortful Information Processing}

Controlled Information Processing

\section{Effortless Processing}

Automaticity in Memory

\section{Ego goals}

- Goal Theory/Goal Setting 


\section{Ego State Theory: Utilization of Dreams}

\author{
STEFANIE BADENHORST
}

Ego State Therapy Training Unit South Africa, Strand, Western Cape, South Africa

\section{Synonyms}

Application; Working with dreams, or the elements within dreams

\section{Definition}

Utilization of Dreams in ego state therapy is where the person assigns identity to the various elements (parts) of the dream and then works with the elements (parts) by engaging with them in dialogue to facilitate communication between parts that are disconnected from each other, resulting in connections and integration of the parts. This resolves the trauma in a gentle and nonthreatening way.

\section{Theoretical Background}

\section{Utilization of Dreams in Ego State Therapy}

John and Helen Watkins (1997) integrated $\checkmark$ hypnotherapeutic techniques with $>$ psychodynamic concepts in the development of ego state therapy. Although ego state therapy has a psychoanalytic rationale, it achieves significant, permanent results in a comparatively short time.

Many early contributors discovered that the human personality is not a unity, but that the personality is separated into various segments, unique entities with different purposes. Janet (1907) applied the term - dissociation to describe ideas clustered together, yet separated from other parts within the personality (as cited in Watkins and Watkins 1997, p. 25). Jung (1969) describes the parts of the personality as a complex of unconscious ideas grouped together. He also described certain more permanent covert structures within the "collective" or "racial unconscious" that he called archetypes. With both terms he implied "personality segments that were organized into unconscious patterns" (as cited in Watkins and Watkins 1997, p. 25). Fedren (1952) was the first to apply the notion of ego states in the psychodynamic explanation of behavior (as cited in Watkins and Watkins 1997, p. 25).

An ego state may be defined "as an organized system of behavior and experience whose elements are bound together by some common principle, and which is separated from other such states by boundaries that is more or less permeable" (Watkins and Watkins 1997, p. 25).

We all have different parts (ego states) in ourselves; you are a child, a parent, a therapist, you can be creative, dynamic, angry, or depressed. There can be between 5 and 15 ego states within a personality. Usually a person is aware of most of the ego states, but some of these states can also be hidden. Ego states can develop by one or more of the following processes: normal differentiation, an introjection of a significant other, or a reaction to trauma or when no other ego state exists to fulfill a specific role (Emmerson 2003, p. 6-12). In the case of a well-adjusted person (healthy) the boundaries between the parts are permeable, but the more rigid the boundaries become, the more severe the pathology.

Figure 1 demonstrates that the ego state range from a healthy, well-adjusted person who is aware of the different parts in the self to a person with pathology or entrenched behavior. As the boundaries between the parts become more rigid, the more difficult it is to reach or engage with those parts that we call ego states. Boundaries between the parts become more rigid as a result of repetitive trauma. The range from rigidity to entrenched behavior can be seen in other personality disorders or severe mental illnesses.

Ego state therapy is usually applied by locating the state that is harboring the pain or trauma and allowing expression of emotions and mastery, and to find states that can be of help or support. The use of dreams in ego state therapy is another way of facilitating connection between parts that are disconnected from each other.

The value of dreams is widely recognized in literature as far back as 1865 . Dreams are unconsciously motivated and appear during some altered state of consciousness, such as sleep (Watkins 1992, p. 103). Dreams can be used in many different ways. Freud valued dreams as the point of departure for free association, others would rather interpret the dream and attach certain meaning to symbols in the dream. Jung (1965, p. 18) valued dreams as part of the prima materia of his scientific work. His own dreams served 
EGO STATES RANGE
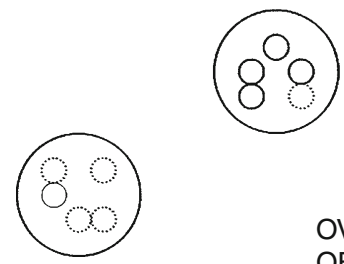

OVERANXIOUS

OBSESSIVE
BEHAVIOR IS

ENTRENCHED
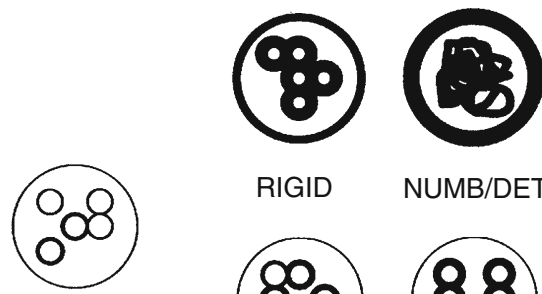

RIGID

NUMB/DETATCHED
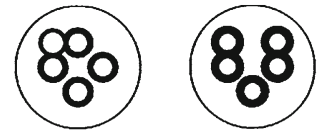

DID

BEHAVIOR IS PATHOLOGICAL

\section{HEALTHY \\ BEHAVIOR CAN BE MODIFIED}

BEHAVIOR IS JUSTIFIED OR SERVES TO PROTECT

Ego State Theory: Utilization of Dreams. Fig. 1 BPD Borderline Personality Disorder, DID Dissociative Identity Disorder

as inspiration to utilize the persons own frame of reference in the healing process. Dreams are often the unconscious surge for solutions of inner conflict. The value of working with dreams is that the information can stay at an unconscious level. The dream may make sense on a conscious level, but it is not essential for the healing process. Overwhelming emotional trauma that is to threatening to deal with, on a conscious level, can be processed at an unconscious level without retraumatization.

The utilization of dreams in ego state therapy has been borrowed strongly from gestalt therapy. The different elements in the dream are viewed as parts of the self and are dealt with as separate entities or ego states. Hypnosis is a tool that can enhance the utilization of dreams. Watkins (1992, p. 28) describes hypnosis as a state of regression, to earlier patterns of behavior and thought "a regression in the service of the ego." Hypnosis is a natural phenomena and the more general explanation is "an altered state of consciousness" or "a focus of attention." Although hypnosis is not essential for utilization of dreams in ego state therapy, a deep trance can allow access to underlying states. Underlying states are traumatized parts holding unresolved emotions. Emmerson (2003, p. 36) agrees that hypnosis allows access to underlying states often not accessible in an ordinary conscious state or a light trance state.
Therefore hypnosis is a valuable tool in working with dreams.

Repetitive dreams or dreams with a repetitive theme are often useful to work with. In the process of understanding, giving support, and being a sponsor for a specific part, the process allows for communication and negotiation between the different parts. The goal in utilizing dreams and ego state therapy lies in understanding and allowing cooperation between the different parts. The art lies in the gentle way of connecting between parts without further trauma.

The utilization of dreams is a valuable tool in conjunction with other techniques in the therapeutic process to facilitate rapid and permanent healing.

\section{Important Scientific Research and Open Questions}

Utilization of dreams in ego state therapy is a relatively new concept, which has been widely presented but as yet not published.

\section{Cross-References}

- Analytical Psychology and Learning

- Dreaming as Consolidation of Memory and Learning

- Family Therapy and Learning

- Freud, Sigmund (1856-1939) 
- Hypnosis and Sleep Learning

- Jungian Learning Styles

- Learning and Recall Under Hypnosis

\section{References}

Emmerson, G. (2003). Ego state therapy. Wales: Crown House Publishing Ltd.

Fedren, P. (1952). Ego psychology and the psychoses. New York: Basic Books.

Janet, P. (1907). The major symptoms of hysteria. New York: Macmillan.

Jung, C. G. (1965). Memories, dreams, reflections. New York: Vantage Books.

Jung, C. G. (1969). The collected works of C. G. Jung. Princeton: Princeton University.

Watkins, J. G. (1992). Hypnoanalytic techniques. New York: Irvington Publishers, Inc.

Watkins, J. G., \& Watkins, H. H. (1997). Ego states theory and therapy. New York: W.W. Norton.

\section{Ego-Involved Goals}

Goal Theory/Goal Setting

\section{Egological self}

Development of Self-consciousness

\section{Elaboration}

In cognitive theories of learning, elaboration involves connecting new information with related information, often stored as prior knowledge. When new information is mentally connected to related information, it can be stored in terms of a more inclusive concept. More cues are then available for retrieval, thus facilitating the effective storage and retrieval of new information.

\section{Elaboration Effects on Learning}

\section{Richard Hamilton}

School of Teaching, Learning and Development, University of Auckland, Auckland, New Zealand

\section{Synonyms}

Elaborative strategies; Integration strategies

\section{Definition}

Elaboration is a cognitive learning strategy that involves any enhancement of information that clarifies or specifies the relationship between information tobe-learned and related information, i.e., a learner's prior knowledge and experience or contiguously presented information. The addition can be an inference, an example, an analogy, a detail, an image, an overall summary, or any other mental construction. Essentially, elaboration is encoding the original content in a different but related way. There are primarily two kinds of elaboration: visual and verbal. For example, to learn the pair "cow-ball" a person could form a visual image of a cow kicking a ball. Alternatively, someone could create a sentence such as "The cow ran after the ball." Elaborations can also differ in terms of source, i.e., in many instructional situations, learners are asked to generate the elaborations themselves. In contrast, relevant elaborations can be built into instructional materials in order to facilitate the learning of the materials.

\section{Theoretical Background}

Historical and current psychological views situate the influence of elaboration on learning within the context of the traditional multistore, multistage theory of memory (e.g., sensory, working and long-term memory) and cognitive processing (Atkinson and Shiffrin 1968). This view of memory suggests that information is processed through a series of memory stores, each with different purposes and characteristics (duration, capacity, etc.). Elaboration strategies facilitate the processing of information as it moves through these memory stores and eventually adds to and/or modifies an individual's knowledge of the world. Elaboration 
strategies allow learners to access and to create organized units of knowledge while processing new information that reduces the overall load on the active memory store, hence increasing the efficiency of the learning process, while also increasing its effectiveness by enhancing the comprehension of incoming information. This increase in efficiency and effectiveness of the learning process consequently facilitates both the creation and/or encoding of new information and skills in people's permanent memory store and increases the likelihood of retrieval of this information or skills when needed.

Elaboration, however, is only one of a set of processing strategies that have been identified as supporting the processing, and eventual learning of new information and skills (Mayer 1989). Elaborative strategies are considered one of the more powerful of learning strategies and a critical component to making

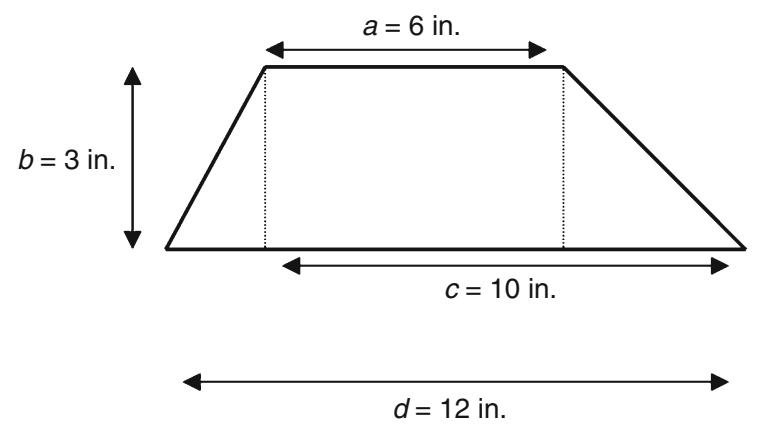

Step 1: Break down the trapezoid into simple figures - two triangles and rectangle. The dotted lines ate perpendicular to the longest side and the horizontal lines are parallel to each other.

Step 2: Find the area of the rectangle and the triangles.

Area of rectangle $=$ width $*$ height

$$
a * b=6 \text { in. } * 3 \text { in. }=18 \text { in. }^{2}
$$

Area of triangle $=[$ base $*$ vertical height $] / 2$

Left triangle $=[(d-c) * b] / 2=[(12-10)$ in. $* 3$ in. $] / 2=3$ in. $^{2}$

Right triangle $=[(c-a) * b] / 2=[(10-6)$ in. $* 3$ in. $] / 2=6$ in. $^{2}$

Step 3: Add the area of the rectangle with the areas of the triangles to get the area of the trapezoid.

$$
18 \text { in. }^{2}+3 \text { in. } .^{2}+6 \text { in. } .^{2}=27 \text { in. }{ }^{2}
$$

Elaboration Effects on Learning. Fig. 1 Workedexample - finding the area of a trapezoid new information meaningful, comprehensible, and consequently, memorable.

\section{Important Scientific Research and Open Questions}

Elaboration was first studied in the context of - paired-associate learning tasks (Rohwer 1973). Paired-associate learning is an important variety of learning that occurs in schools and, thus, has been extensively studied in educational research. Examples of school-learning tasks involving paired-associate learning include learning the names of new objects, learning definitions, learning the capital cities of countries, etc. Elaborations have consistently been found to facilitate retention of target verbal information (Levin 1988). In addition, researchers have found that elaboration can have significant positive effects on more complex outcomes, e.g., skill and concept application, problem-solving. The effectiveness of elaborations is differentially moderated by the prior knowledge or level of expertise of the learner, source of the elaboration (self- or instructionally generated), the learning outcome, the type of elaborations, and the structure of the instructional material.

Explanations for the influence of elaboration on learning assume that people's knowledge is organized into networks of concepts, experiences, and beliefs (see $>$ schema and $\triangleright$ mental models). The specific explanations differ depending on the nature of the learning outcome. For retention of verbal or visual information, elaborative activities may increase the richness or the distinctiveness of the encoded memory episode. "Richness" within this context is operationalized as the number of interconnections between the target propositions and a learner's related prior knowledge. At the time of recall, learners will typically activate only a small subset of the original propositions and attempt to reconstruct these propositions. The richer and more redundant the activated subset of propositions, the more likely the reconstruction of the original set of propositions. An alternative explanation for the positive effects of elaboration may be found in the research which focuses on the distinctiveness of encoded episodes. Research suggests that distinctive encoding can be described as the processing of differences among the items of an episode. That is, focusing on the differences among the units of a memory episode should increase the distinctiveness 
of the episode and increase the probability that the set of propositions will be more easily found and accessed during recall and retrieval situations. An example of a "richness" elaborative activity is one that asks a learner to identify how the to-be-remembered information relates to prior personal experiences. Alternatively, an example of a "distinctiveness" elaborative activity is one that asks a learner to identify how the different pieces of the to-be-remembered information fit together.

The positive effects of elaboration to facilitate more complex learning outcomes often focuses on its value in facilitating the development of appropriate mental models which allow increased understanding of and application of target concepts, principles and skills. In creating knowledge related to new domains or areas, people often create "mental models" which approximate important ideas and principles within these new domains. Well-elaborated mental models allow learners to identify critical aspects of new problems and tasks which in turn support superior problem-solving and application (Mayer 1989).

Current research on the effects of elaboration on remembering information is attempting to tease out how the nature of to-be-learned material (e.g., text coherence, authenticity), the nature of elaborative strategies (e.g., self-generated versus author-generated), and the prior knowledge and motivational disposition of a learner interact to influence the effectiveness of elaborative strategies (cf. Dornisch et al. 2011). An important focus within the research on more complex outcomes, e.g., problem-solving and related skills, has been on assessing the value of different elaborative strategies (e.g., $>$ self-explanation, - worked examples) on acquiring important complex learning outcomes. In general, prompted and spontaneous self-explanations and the presentation of worked examples have been found to improve the learning of problem-solving skills, particularly for low-knowledge learners. Given these positive effects, researchers are focusing on how best to develop materials to support self-explanation and the development of effective worked examples (cf. Kalyuga 2009).

\section{Cross-References}

- Cognitive Learning

- Cognitive Load Theory
Elaboration Strategies and Human Resources

Development

- Human Information Processing

- Imagery and Learning

- Learning Strategies

- Memory Structures

- Mental Models

- Schema(s)

\section{References}

Atkinson, R. C., \& Shiffrin, R. M. (1968). Human memory: A proposed system and its control processes. In K. W. Spence \& J. T. Spence (Eds.), The psychology of learning and motivation (Vol. 2, pp. 89-195). New York: Academic.

Dornisch, M., Sperling, R. A., \& Zeruth, J. A. (2011). The effects of levels of elaboration on learners' strategic processing of text. Instructional Science, 39(1), 1-26.

Kalyuga, S. (2009). Knowledge elaboration: A cognitive load perspective. Learning and Instruction, 19, 402-410.

Levin, J. R. (1988). Elaboration-based learning strategies: Powerful theory = powerful application. Contemporary Educational Psychology, 13(3), 191-205.

Mayer, R. (1989). Models for understanding. Review of Educational Research, 59, 43-64.

Rohwer, W. D., Jr. (1973). Elaboration and learning in childhood and adolescence. In H. W. Reese (Ed.), Advances in child development and behavior. New York: Academic.

\section{Elaboration Strategies and Human Resources Development}

\author{
Thomas N. Garavan ${ }^{1}$, Fergal O’Brien ${ }^{2}$ \\ ${ }^{1}$ Department of Personnel and Employment Relations, \\ Kemmy Business School, University of Limerick, \\ Limerick, Ireland \\ ${ }^{2}$ Department of Accounting and Finance, Kemmy \\ Business School, University of Limerick, \\ Limerick, Ireland
}

\section{Synonyms}

Cognitive learning strategies

\section{Definition}

Elaboration theory is an instructional design theory that argues instruction should be organized in 
increasing order of complexity for optimal learning, while providing a meaningful context in which subsequent ideas can be integrated. It was pioneered by Charles Reigeluth and colleagues at Indiana University in the late 1970s.

Learning strategies are behaviors or thoughts that facilitate learning (Weinstein and Mayer 1986). An elaboration strategy is defined as a process by which the learner builds an internal connection between what is being learned and previous knowledge. They range from simple study skills, such as highlighting important text, to complex thought processes, such as employing analogies. Elaboration strategies include mnemonics to help with memory tasks, summarizing, paraphrasing, creating analogies, generative note taking, question-asking, question-answering, and selecting key ideas from texts (Pintrich 2002). Such strategies result in deeper processing of the learning material and better comprehension and learning. In other words employing an elaboration strategy involves attaching a symbolic construction to the learning material in order to make it more personally meaningful. The elaboration strategy leverages prior knowledge to facilitate further learning. "Prior knowledge" includes previous learning, experiences, attitudes, beliefs, and values.

\section{Theoretical Background}

The paradigm shift from teacher-centric instruction to learner-centered instruction has caused "new needs for ways to sequence instruction" (Reigeluth 1999). According to Reigeluth (1999), the theory of elaboration is characterized by the following:

- It values a sequence of instruction that is as holistic as possible, to foster meaning-making and motivation.

- It allows learners to make many scope and sequence decisions on their own during the learning process.

- It is an approach that facilitates rapid prototyping in the instructional development process.

- It integrates viable approaches to scope and sequence into a coherent design theory.

Wilson and Cole (1992), summarize the basic strategies of elaboration theory as follows:

1. Organizing structure: conceptual, procedural, or theoretical
2. Simple-to-complex sequence of lessons

3. Within-lesson sequencing:

(a) For conceptually organized instruction "present the easiest, most familiar organizing concepts first."

(b) For procedures, "present the steps in order of their performance."

(c) For theoretically organized instruction, move from the simple to the complex.

4. Summarizers: content reviews at both lesson and unit levels

5. Synthesizers: For example, diagrams that help the learner integrate content elements into a meaningful whole

6. Analogies: relate the content to learner's prior knowledge

7. Cognitive strategy activators: cues that can trigger cognitive strategies for appropriate processing of materials

8. Learner control: allow learners to exercise informed control over both content and instructional strategy

With all learning- and knowledge-gaining processes in organizations, there is a problem of integrating new knowledge into an existing cognitive structure. Organizations increasingly expect employees to acquire significant amounts of new knowledge in very short periods of time. Human resource development (HRD) specialists and instructional designers are continuously looking for ways to accelerate the learning process and ensure that employees reach experienced worker standards in the shortest time possible. A large proportion of training which takes place in organizations is of a technical variety requiring both novice and experienced learners to quickly integrate this new knowledge into their working practices. The majority of training in organizations tends to focus on cognitive or knowledge-based objectives rather than skill or attitude objectives. Examples include induction training, health and safety training, and technical and regulation training. One strategy that can be used by trainers to accelerate knowledge acquisitions is through the use of elaboration strategies.

Van Dijk and Kintsch (1983) recognized that many learners experience problems in integrating new knowledge into their existing knowledge store. They suggested that learners could be facilitated to understand and remember new knowledge through the use of 
strategies that directly link new knowledge to existing knowledge. Elaboration strategies therefore encourage new learning through understanding.

Elaboration strategies fit into the broader category of cognitive learning strategies. McKeachie et al. (1986) propose a taxonomy of cognitive learning strategies consisting of rehearsal, elaboration, and organizational strategies. Rehearsal strategies are typically employed by learners to remember training materials using repetition. This might include repeating the material aloud, copying the material, taking selective verbatim notes, and underlining the most important parts of the training material. Organizational strategies focus on how learners organize and build connections with the information received in a learning situation. They may do this through diagramming, networking, and outlining (Weinstein and Mayer 1986). Elaboration strategies differ from the other two cognitive strategies in that the focus is on the connection of ideas and the integration of this new knowledge into existing knowledge. The research evidence suggests that cognitive learning strategies are important in explaining learner performance in e-learning settings. In particular they have application to a variety of learning tasks ranging from simple tasks such as the recall of information, words, or lists to more complex tasks that require comprehension of the information, for example, understanding the sequence of tasks in a process.

The use of elaboration strategies in instructional settings can be approached in two ways: providing learners with preconstructed elaborations or asking learners to generate their own elaborations. Preconstructed elaborations appear to be effective in facilitating memory of key facts. However, generating elaborations are more effective for adults because they encourage learners to access their knowledge base and make connections between what they already know and what they have to learn. The knowledge base of the learner appears to be fundamental in the successful use of a generating elaboration strategy. This approach produces fewer learning gains with novice learners.

The use of learning strategies is strongly supported by research on how the brain operates. Tomlinson (1999) states that "the brain is physiologically predisposed to connect parts to wholes." This suggests that the brain looks for meaningful patterns in knowledge. The challenge for the instructional designer is to create a situation where learners can make such connections. Elaboration strategies if used appropriately allow such connections to be made. In an extensive review of cognitive learning theory, Derry (1990) argued that new information is connected to prior knowledge in working memory through establishing connections using instructional strategies that illustrate this connection.

Derry (1990) argues that elaboration strategies facilitate the conversion of "comprehended information" into "learned information." The knowledge learned can then be labeled meaningfully by the learner because of the rich connections made with existing knowledge. Elaboration strategies facilitate crossreferencing and the possibility to access other knowledge to which it is connected.

\section{Important Scientific Research and Open Questions}

In general there is a paucity of research on the use of elaboration strategies in industrial and organizational training situations. Human resource development specialists frequently utilize short intensive class room training strategies combined with online and blended learning programs. They also train learners with varying degrees of knowledge ranging from novice to expert. These variegated learning situations impose particular challenges on trainers and instructional designers to achieve optimal learning outcomes. There is also an increased focus on self-directed learning. Here learners have to become proficient in learning how to learn and to make good choices as to the strategies they employ to maximize their learning. In a society and work context where knowledge and information are constantly changing, there is a significant burden placed on learners and HRD specialists to accelerate the learning process.

Given that elaboration strategies have primarily been studied in educational rather than industrial training settings a number of key questions remain unanswered.

1. How effective is the use of different learning strategies with learners that are novices compared to learners that are experts?

2. How do elaboration strategies used in conjunction with other cognitive, metacognitive, and resource strategies work in both classroom and e-learning settings? 
3. What types of elaboration strategies work best in technical training situations? Specifically, how effective are the use of images, spontaneous chains of association, and memory training strategies?

4. What is the best way to develop the skills of trainers and instructors in the use of elaboration strategies? And how can learners become proficient in selecting the most appropriate learning strategy to suit the learning challenge?

The characteristics of individual learners in the context of the use of elaboration strategies in workplace training settings are not well understood. Three specific questions remain to be conclusively answered.

1. What influence does the self-efficacy beliefs of learners concerning their performance capabilities influence the use of elaboration strategies? For example, in an educational context it has been shown that students with high self-efficacy were more cognitively involved in learning new material.

2. To what extent do the task value beliefs of learners impact their use of elaboration strategies? For example, individuals will likely have perceptions concerning how important the learning is, its utility value in their role or job, and their personal interest in the task. Where individuals are high on these three dimensions they will make greater use of elaboration strategies.

3. What is the role of learner goal orientation, in particular, mastery goal orientation in influencing the use of elaboration strategies? It is likely that high mastery goal orientation will lead to greater and more effective use of elaboration strategies.

It is clear that there is much research to be undertaken in corporate settings.

\section{Cross-References}

- Cooperative Strategies

- Elaboration Effects on Learning

- Human Resource Development and Performance Improvement

- Instructional Design

- Learning Strategies

- Metacognitive Strategies

- Organizational Change and Learning

\section{References}

Derry, S. J. (1990). Learning strategies for acquiring useful knowledge. In B. F. Jones \& L. Idol (Eds.), Dimensions of thinking and cognitive instruction (pp. 347-379). Hillsdale, NJ: Lawrence Earlbaum.

McKeachie, W. J., Pintrich, P. R., Lin, Y., \& Smith, D. (1986). Teaching and learning in the college classroom: A review of the research literature. Ann Arbor MI: National Centre for Research to Improve Postsecondary Teaching and Learning, University of Michigan.

Pintrich, P. R. (2002). The role of metacognitive knowledge in learning, teaching, and assessing. Theory into Practice, 41, 219-225.

Reigeluth, C. M. (1999). The elaboration theory: Guidance for scope and sequence decisions. In C. M. Reigeluth (Ed.), Instructionaldesign theories and models: A new paradigm of instructional theory (Vol. II). Hillsdale, NJ: Lawrence Erlbaum Assoc.

Tomlinson, C. A. (1999). The differentiated classroom: Responding to the needs of all learners. Virginia: Association for Supervision and Curriculum Development.

Van Dijk, T. A., \& Kintsch, W. (1983). Strategies of discourse comprehension. New York: Academic.

Weinstein, C. E., \& Mayer, R. E. (1986). The teaching of learning strategies. In M. C. Wittrock (Ed.), Handbook of research on teaching (pp. 315-327). New York: Macmillan.

Wilson, B., \& Cole, P. (1992). A critical review of elaboration theory. Educational Technology Research and Development, 40, 63-79.

\section{Elaborative Interrogation}

Elaborative interrogation (EI) is a questioning technique that requires learners to answer "why" questions. Why questions increase prior knowledge activation, reducing the arbitrariness of the newly learned information.

\section{Elaborative Strategies}

- Elaboration Effects on Learning

\section{eLearning}

- Mobile Learning

\section{e-Learning}

- Computer-Based Learning

- Courseware Learning 
- Integrated Learning Systems

- Interactive Learning Environments

- Learning with and from Blogs

- Online Learning

- Open Learning

\section{e-Learning and Digital Learning}

STEVE WHEeleR

University of Plymouth, Plymouth, Devon, UK

\section{Synonyms}

Technology-enhanced learning; Web-based learning

\section{Definition}

e-Learning - or electronic learning - has been referred to as "technology-enhanced learning," and more recently as "digital learning." e-Learning describes a set of technology-mediated methods that can be applied to support student learning and can include elements of assessment, tutoring, and instruction. Learning using technology is not the same as learning through technology. Learning using technology implies that the technology is being used as one method amongst many others, whilst learning through technology suggests that the technology is the sole conduit through which the student receives instruction and communicates with his instructor. There are of course many media and technologies available to support e-learning. The World Wide Web, for example, can be a communications medium that at once liberates the learner whilst simultaneously bonding him to others, establishing communities of learning (Jonassen et al. 1999).

\section{Theoretical Background}

e-Learning draws on technology that has been seen as "mindtools." Jonassen et al. (1999) argue that students cannot learn from technology much in the same way that they cannot learn from teachers. Rather, students learn from thinking, and technology can be seen as merely a vehicle to aid the process (Clarke 1994). In effect, e-learning can represent this set of mind tools.

Technology such as the personal computer can be used to support learning through exploration, and as a cognitive tool to encourage learners to develop their own thinking and approaches to solving problems. Perhaps one of the best ways computers can be used to support learning is in the promotion of collaborative learning. Collaborative approaches encourage students to negotiate shared meaning and to work as teams rather than competitively toward a common goal. Using online technologies such as the Internet can enable learners to collaborate together even if they are separated by distance.

Collaborative e-learning is a simple prospect when transferred to the e-learning context. Students are encouraged to collaborate asynchronously through online discussion groups in a threaded format or synchronously through chat rooms. The emergence of Web 2.0 (social software) applications in e-learning has been one of the most exciting developments since the birth of the World Wide Web. Social software such as web logs (blogs) and wikis have already started to transform the way people communicate and access knowledge, and they are changing the manner in which we create content and collaborate online. Wikis provide a shared space in which all contributors are readers and writers. Free editable space is provided for users to share their knowledge and build on each others' ideas and learning. This unstructured approach to knowledge generation has few boundaries beyond the control the group wishes to exert, so a kind of psychological safety is engendered, and is strengthened the more users contribute to the Wiki.

Blogs are another means of producing online diaries, and can be incorporated into larger Web sites, or can stand alone. The user regularly posts ideas, thoughts, views, and opinions, and so on to the web space, and can, if $s /$ he wishes, provide a response box for readers to post their return comments. Dialog can be created between people who have never met, but who share a common interest in the topic of the blog. We could explore notions of the self and how it compares to the "electronic self," but in essence, if we believe that all individuals "manage" the impression of themselves that they reveal to others, then we must consider that the blog may be another form of personal performance aligned to Erving Goffman's notion of the "front stage" self. We can then hypothesize about what information the individual hides, disguises, or omits, as he or she writes the blog. Wikis and blogs then have the potential to change forever the manner in which 
students collaborate, and open up a new range of possibilities for online learning within virtual groups.

New tools such as microblogging services (e.g., Twitter, Tumblr) are also being used to promote near-synchronous discussion, sharing of resources, collaboration, and the development of individual Personal Learning Networks (PLNs) which extend the scope and reach of e-learning beyond geographical and cultural boundaries.

As e-learning develops and evolves, we can expect to see the emergence of many new ways to use digital technologies to support and enhance education and training. Mobile technologies and handheld smart devices will become important tools to extend learning beyond the classroom and into just about any context where students are on the move.

\section{Important Scientific Research and Open Questions}

There are many emerging opportunities for research in the field of e-learning and digital learning, many of which can be located within the learner experience. Essentially, a large proportion of research into e-learning is aimed at improving the learner's experience and promoting deeper engagement with content to achieve better learning outcomes.

Technology affordance is an important area of discussion and research in e-learning, particularly within the domain of learning space design. The affordance of a technology relates to user perception of its properties and how these allow it to be used in certain ways. Recent research has been undertaken into how the affordances of e-learning technologies can best match learning tasks and outcomes (Bower 2008).

Learning design is also a key area of research in e-learning. There has been much criticism in recent years over the usability, expressivity, and interoperability of institutional virtual learning environments or VLEs (Griffiths et al. 2009), and efforts to improve the effectiveness of VLE navigation systems. There is also a developing discourse around the notion of PLEs - personal learning environments - and how students can develop their own combinations of digital tools in a personal web configuration to achieve better individualized e-learning outcomes.

The use of digital games applications in e-learning has been a topic for research for some time. Interactive learning environments featuring digital games can take many forms, but in serious game format, clear goals and outcomes are prescribed for students, and the engaging and exploratory nature of the learning can yield positive experiences (de Freitas and Jarvis 2009). The simulation affordances of games are particularly useful for preparing students for the "real world" of work, and in professional practice, games-based simulations can be particularly effective as e-learning strategies. Furthermore, games facilitate engagement of students' audio, visual, kinesthetic, and tactile senses, which can provide for a more immersive learning experience that enables richer acquisition of skills and knowledge in a situated context.

e-Safety is a concern for many actors in the sphere of e-learning. In schools, particular care is taken to protect children from the perceived dangers of the Internet and its associated tools and services. Social networking, for example, has been the focus of much media attention in recent years, due to the dangers of children unwittingly conversing with undesirable or dangerous adults posing as children or exposure to dangerous or inappropriate material. There are also issues of cyber bullying, invasion of privacy, and theft of identity that have prompted research to be conducted into discovering ways to protect users and make the social web a safer environment to learn within. These issues affect adult and child learners alike, and can be seen as emergent properties of an imperfect society that is increasingly connected through social media.

Another area of study surrounds the skills and knowledge students bring with them to e-learning. Often referred to as "digital literacies," these skills actually encompass an entire spectrum of new life skills that include how to discern between the good and the bad sources on the Internet, knowing the appropriate ways to converse with others when social cues are reduced, and generally how to relate to a digitally driven world. Emerging practices in student-generated content for e-learning include blogging, video creation and personal broadcasting, new writing practices, repurposing and remixing of content, and the creation of new multimodal artifacts. All are valid topics within the field of digital literacy research (Lankshear and Knobel 2006).

Perhaps the most prevalent topic for e-learning research surrounds the notion of community. e-Learning is becoming increasingly collaborative and 
cooperative in nature, as more socially facilitative tools emerge on the Internet. Researchers are now concentrating their efforts into defining communities of practice and interest, investigating what constitutes a personal learning network, and studying the effects of rich social media on learning. Ultimately, all e-learning studies should concentrate more on the learning than on the technology however. Doing so should avoid techno-deterministic solutions in favor of the development of tools that support the needs of the learner.

\section{Cross-References}

- Advanced Learning Technologies

- Distance Learning

- Distributed Technologies

- General Literacy in a Digital Work

- Learning Technology/Technologies

- Literacy and Learning

- Socio-Technological Change of Learning Conditions

- Technological Learning in Organizations

- Web Science

\section{References}

Bower, M. (2008). Affordance analysis: Matching learning tasks with learning technologies. Educational Media International, 45, 3-16.

De Freitas, S., \& Jarvis, S. (2009). Towards a development approach to serious games. In T. Connolly, M. Stansfield, \& L. Boyle (Eds.), Games-based learning advancements for multi-sensory human computer interfaces. Hershey: IGI Global.

Griffiths, D., Beauvoir, P., Liber, O., \& Barrett-Baxendale, M. (2009). From reload to recourse: Learning from IMS design implementations. Distance Education, 30, 201-222.

Jonassen, D. H., Peck, K. L., \& Wilson, B. (1999). Learning with technology: A constructivist perspective. Upper Saddle River: Prentice Hall.

Lankshear, C., \& Knobel, M. (2006). New literacies: Everyday practices \& classroom learning. Maidenhead: Open University Press.

\section{E-Learning Authoring Tools}

\section{Marcus Specht}

Center for Learning Sciences and Technology, Open

Universiteit Nederland, Heerlen, The Netherlands

\section{Synonyms}

Authoring tools; Hypermedia authoring; Learning object creation; Metadata

\section{Definition}

E-learning authoring tools enable users to create learning content for delivery or reuse. In general authoring tools range from creating and editing digital assets to tools that create instructional designs, complex simulations, games, or tutoring components. All authoring tools have the goal in common to offer dedicated support for authors to create an educational product on a higher level of abstraction. Therefore, they offer basic concepts for structuring the educational product as content components, elements, templates, or scripts to enable the efficient development of e-learning products. Furthermore, in most cases they either are integrated with a delivery system as a Learning Management System (LMS) or offer exporting and conversion functionality to different content exchange standards or interoperable technical formats.

\section{Theoretical Background}

E-learning authoring tools aim at making the creation of educational products and e-learning content more efficient. Production of e-learning products can range from single assets, through course components, up to complete instructional designs. As soon as multimediaand hypermedia-based educational content became prominent, authoring of components became essential to enable educators and instructional designers to create their own products with little technical support. In the last 20 years, the way content has been designed, created, reused, and meta-tagged has changed dramatically. On the one hand, different tendencies have fostered the creation of authoring tools for end users rarely working with instructional design and content creation; on the other hand, tools for teams of experts and instructional designers have been developed to support professional workflows of educational content production. Creation of content has also always been closely related to the formats and methods of content delivery and nowadays content packaging standards and specifications enable the exchange of learning content between most LMS, Learning Content Management Systems (LCMS), and courseware platforms (Falvo and Johnson 2007; Sampson and Karampiperis 2006).

The development of web-based interfaces has enabled the social creation of content in distributed authoring networks. In the 1990s, still the most used method for creating learning content was the use of 
local authoring applications as Macromedia Authorware, Macromedia Director, or Toolbook. These locally installed applications offered a template system and enabled the easy creation of multimedia elements from a local file system as also connecting these components via a learning logic. From the late 1990s onward, the development of web-based authoring solutions and the support of distributed authoring environments became more and more popular. This led to the development of distributed authoring solutions enabling creation, sharing, reuse of content packages, and even single courseware components. The development of social software and its use in education also had an impact on authoring tools. By empowering learners to be content creators new ways of creating, referencing, and sharing learning content has developed in the last 10 years. In the following, some of the conceptual aspects and criteria to differentiate e-learning authoring tools will be highlighted.

- Complexity of product: E-learning authoring tools support a wide variety of authoring activities ranging from asset creation, reusable content package creation, up to instructional design authoring. Furthermore, a wide variety of e-learning content types must be considered from multimedia and hypermedia content, interactive simulations, quiz and assessment components for which specialized tools have been developed. Cisco's Reusable Information Object Strategy Whitepaper (Barritt et al. 1999) was one of the first taxonomies that combined a conceptual content structure, educational goals, and guidelines for authors. Instructional design tools enable authors to create combinations of learning activities, learning environments, and a learning logic on different levels of complexity. To support the reuse and exchange also specifications and interoperable formats as IMS-LD (Koper and Olivier 2004) have been developed. New approaches also support the development of highly complex simulations and serious games with end user editing tools while a prominent approach of handling complexity in such tools is the use of higher-level template systems and component-based authoring.

- Integration with product delivery: For integrating e-learning authoring tools into the complete e-learning cycle, different approaches have been established in industry and research. Most major
LMS platforms offer integrated authoring tools for creation of content, and assessment components. Furthermore, there is a wide variety of stand-alone authoring tools that support single authors or complete teams of authors working in distributed workflows. Most authoring tools support the integration of different asset formats ranging from different text, graphic, animation, and audio formats. Especially the development of content packaging and exchange specifications as SCORM, AICC, or IMS content packaging enabled high flexibility to use different authoring tools which create a content package that can be integrated into a learning management system. Nowadays most Learning Management Systems (LMS) and Learning Content Management Systems (LCMS) support the import and export of content packages in different formats. Cross-platform content publishing is an ongoing research thread that is confronted with new formats and ways to publish and distribute content continuously. In this thread, the development of federated learning object repositories has highlighted the importance of metadata standards as IEEE LOM and their role in sharing and reuse of learning content.

- Conceptual models for authoring: Different models for content creation and authoring have been developed on the basis of instructional design models as ADDIE (Dick and Carey 1996). In these models different phases of analysis, conceptual design, interaction design, development, implementation, and evaluation have been specified. Adaptive Educational Hypermedia Authoring tools have been developed further to enable the dynamic linking of content and conceptual knowledge spaces (Brusilovsky 2003). Adaptive educational systems and the authoring of those is still an ongoing research track in more dynamic for example rulebased systems are used for the combination of learning content in real time. Most authoring tools today support the authoring of learning content, specific sequencing logics, as also services and interaction components.

\section{Important Scientific Research and Open Questions}

Currently, several worldwide initiatives for open educational resources (OER) are the most influential 
developments for authoring and creation of learning content. While this has led to a variety of tools for rapid content production and packaging, still questions of appropriate business models are highly discussed. The combination of high-quality authored content packages and user-generated content as also complementary services for content packages as tutoring, assessment, and certification are under discussion. In general, these initiatives have led to a more user-driven content production and open content corpuses as WIKIs or educational blogs.

The flexible delivery of authored educational products has always been a challenge and still is both on the conceptual and on the technical level. On the technical side, most problems for cross-platform content development and delivery have been solved for personal computers. Nevertheless, with the trend toward mobile content access and mobile learning support new challenges arise in cross-platform delivery. For the technical problems of delivering learning content to mobile devices as smartphones or tablet computers a variety of solutions are under development. Authoring content for blended or ubiquitous learning scenarios and delivery is one of the current research challenges as both the technical questions of delivering and converting content for cross-platform delivery is challenging as also the conceptual problems of the instructional design and the orchestration of learning services is highly discussed.

Authoring tools for more complex educational products and rich media environments as Intelligent Tutoring Systems (ITS), instructional designs in IMS$\mathrm{LD}$, educational games, or virtual worlds are recently under discussion. In general, one approach is to wrap the complexity of educational logics and products into template systems and empowering end users to customize scripts or templates to their educational domain. Furthermore the developments of Personal Learning Environments (PLE) building on Web 2.0 technologies is researching a more learner-directed approach for creating learning environment via the integration of learning content and services in Mashup environments.

\section{Cross-References}

- e-Learning and Digital Learning

- Learning Management System

- Trust into e-Learning

\section{References}

Barritt, C., Lewis, D., \& Wieseler, W. (1999). Cisco systems reusable information object strategy. Definition, creation overview, and guidelines version 3.0. http://www.cisco.com/warp/public/779/ ibs/solutions/learning/whitepapers/el_cisco_rio.pdf. Accessed 9 Jan 2010.

Brusilovsky, P. (2003). Developing adaptive educational hypermedia systems: From design models to authoring tools. In T. Murray, S. Blessing, \& S. Ainsworth (Eds.), Authoring tools for advanced technology learning environment (pp. 377-409). Dordrecht: Kluwer.

Dick, W., \& Carey, L. (1996). The systematic design of instruction (4th ed.). New York: Haper Collins College Publishers.

Falvo, D. A., \& Johnson, B. F. (2007). The use of learning management systems in the United States. TechTrends, 51(2), 40-45.

Koper, R., \& Olivier, B. (2004). Representing the learning design of units of learning. Educational Technology \& Society, 7(3), 97-111.

Sampson, D. G., \& Karampiperis, P. (2006). Towards next generation activity-based learning systems. International Journal on ELearning, 5(1), 129-149.

\section{e-Learning Data Analysis}

Educational Data Mining

\section{Electronic Threaded Discussion}

Asynchronous Learning

\section{Elements of Movement}

Impaired Multidimensional Motor Sequence
Learning

\section{E-Literacy}

General Literacy in a Digital World

\section{Embedded Questions}

$\checkmark$ Learning from Questions 


\section{Embodied Agents}

Cognitive Modeling with Multiagent Systems

\section{Embodied Cognition}

\author{
Mauro Adenzato, Francesca Garbarini \\ Department of Psychology, Center for Cognitive \\ Science, University of Turin, Turin, Italy
}

\section{Synonyms}

Embodied embedded cognition; Embodied mind; Embodiment

\section{Definition}

The word embodied refers to the dual valence of the notion of body: bodiness is a combination of a physical structure (the biological body) and an experiential structure, which corresponds to the living, moving, suffering, and enjoying body. From here it is possible to arrive at the dual acceptation of embodied cognition, which refers, on the one hand, to the grounding of cognitive processes in the brain's neuroanatomical substratum, and on the other, to the derivation of cognitive processes from our organism's sensorimotor experiences.

The paradigm of embodied cognition is progressively asserting itself in the domain of Cognitive Science: the mind is no longer conceived as a set of logical/abstract functions, but as a biological system rooted in bodily experience and interconnected with bodily action and interaction with other individuals. From this perspective, action and representation cannot be interpreted in terms of the classic physicalmental state dichotomy, but are closely interconnected. Acting in the world, interacting with objects and individuals in it, representing the world, perceiving it, categorizing it, and understanding its significance are simply different levels of the same relational link that exists between organisms and the local environments in which they operate, think, and live.

\section{Theoretical Background}

The founding principle of the cognitive sciences was that of a clear distinction between function and organ.
This conceptual approach has found its most wellknown expression in the philosophical current of functionalism, which holds that, although a process may be carried out by a biological substrate system (such as the brain) or by an electronic substrate system (such as a computer), this does not necessarily imply any difference between the two processes. This is the principle of multiple realizability, which rests on the assumption that the brain is a syntactic information processor, equivalent to a digital calculator or to any other physical system that can be described in computational terms. In recent years, the cognitive sciences have progressively gone beyond the functionalist metaphor by acknowledging that cognitive processes are rooted in the neuroanatomic substrate and by describing the mind as an emerging property of the brain. The paradigm of embodied cognition has increasingly asserted itself among this second generation of cognitive sciences, referring to both the embedding of cognitive processes in brain anatomy and the origin of these processes in an organism's sensorimotor experience.

One historical antecedent of the modern paradigm of embodied cognition is undoubtedly the concept of affordance, a neologism created by the psychologist James Gibson to refer to the opportunities that objects in the environment offer ("afford") for interaction to an organism's various sensorimotor capacities. The central point of Gibson's theory was his explicit refusal of the dichotomy between action and perception and the underlying dualism between physical and mental capacities. Perception and action were thought to be directly coupled, with no processing of sensory information and no subsequent conversion into motor input. The motor interaction schema was considered to be specified already in the content perceived, as an integral part of it.

Gibson's pioneering efforts and his ecological perspective certainly represent a fundamental antecedent for the paradigm of embodied cognition, which is steadily making headway in the panorama of cognitive science. Lakoff and Johnson (1999) effectively described this progressive mutation of the cognitive science paradigm, by distinguishing between firstgeneration and second-generation cognitive science, defining them disembodied mind and embodied mind, respectively. The first generation of cognitive science coupled the computational metaphor of cognitive processes as software - independent of cerebral 
hardware - with an abstract conception of reason, which, in a Cartesian way, was considered as being independent from the body and its activity. Conversely, the central point of second-generation cognitive science is represented by close interaction between mind and body, between thought and action, between rational schemas and sensorimotor schemas.

Therefore, second-generation cognitive science differs from the first, not only in its refusal of computational functionalism, but also in the actual conception of its subject, human cognition. Instead of abstract mental processes, which are describable in formal terms of logic, cognitive processes are considered in the light of their intrinsic ties to the body's action and sensorimotor experience. This is a crucial aspect of the issue, underscored earlier by Gibson.

Varela et al. (1991) identified the matrix of the concept of embodied in the phenomenology of Merleau-Ponty and clarified the dual valence of the concept of embodied in expounding their theory of cognition and embodied action: "By using the term embodied we mean to highlight two points: first, that cognition depends upon the kinds of experience that comes from having a body with various sensorimotor capacities, and second, that these individual sensorimotor capacities are themselves embedded in a more encompassing biological, psychological, and cultural context" (pp. 172-173). The authors also clarify the term action by affirming that sensory and motor processes, perception, and action are fundamentally inseparable in lived cognition. For biological organisms, action and perception "are not merely contingently linked in individuals; they have also evolved together" (p. 173).

\section{Important Scientific Research and Open Questions}

Recent discoveries in the neurophysiological domain have provided decisive evidence for the paradigm of embodied cognition and have experimentally confirmed the existence of a mechanism (the Gibson's affordance) by which an object's shape and function are coupled and perceived directly by an observer (Garbarini and Adenzato 2004). In a series of studies conducted over the course of the last 20 years, Rizzolatti and colleagues (see Rizzolatti and Craighero 2004, for a review) have discovered the existence of two distinct groups of neurons in the premotor cortex of monkeys and humans, both of which fire when specific actions are executed. The two neuron groups differ in the sense that, even if there is no active execution of a given action, one neuron group is activated by the mere observation of an object toward which the action may be directed. The other neuron group is activated by observing another person executing that action with the object. Therefore, both neuron types are bimodal, that is, motor as well as perceptual, because they fire when motor programs are activated, but also during purely perceptual recognition tasks. The activity of these two groups of bimodal neurons can be conceptually captured by referring to a simulation mechanism. Specifically, when an object is under observation, a motor schema appropriate to the characteristics of that object is activated (e.g., for shape, size, and spatial orientation) as if the observer were interacting with it. Similarly, when observing another individual executing an action with an object, the observer's neural system is activated $a$ if it was executing the same action. In both instances, motor activation is only virtual, and the action is not actually carried out, but neurally simulated. The first type of neurons are called canonical neurons, because they are activated by an object's physical features. The neurons in the second group are called mirror neurons, because they induce a specular reaction in an observer's neural system.

In the light of this as if neural simulation mechanism, the motor system's function in the entire cognitive system can now be reinterpreted. Researchers used to attribute an exclusively action planning and execution role to the motor system, but the discovery of the above-described bimodal neurons in the premotor cortex and in the posterior parietal cortex, has provided strong evidence for their implication in the perceptual recognition of objects and actions. Hence, in agreement with the embodiment theory, the neurophysiological approach has confirmed a dynamic dimension in perception and underscored an intrinsic link with the sphere of action. In fact, both perspectives consider interaction with an object a constituent part of the perceptual representation of the object itself.

From the embodied cognition perspective, not only perceptual representation but also high-level cognitive processes, such as concept formation and language, are essentially based on motor programs. Contrary to the classical approach in cognitive science, in which concepts are viewed as amodal and arbitrary symbols, 
embodied semantics argues that concepts must be grounded in sensorimotor experiences in order to have meaning. In fact, it advances the view that concepts are multimodal entities, whose neural representations overlap with those of the individual's perceptual and motor experiences. A concept such as "apple," for instance, would be represented by many of the same group of neurons that underlie the perception of its color, its round shape, its particular taste, weight, consistency, and so on. Retrieving a concept, thus, would be nothing more than simultaneously reactivating the stored traces of past perceptual and motor experiences associated with it.

In line with this view, neuroimaging studies (Aziz Zadeh and Damasio 2008, for a review) have shown a somatotopic pattern of activation along cortical motor areas for the observation of actions involving different body parts, as well as for action-related language comprehension. For example, the concept of "grasping" would be represented in sensorimotor areas that represent grasping actions; the concept of "kicking" would be represented by sensorimotor areas that control kicking actions; and so forth. The signals related to the common goal for a variety of specific actions, that is, grasping with the mouth, or with the hand or toes, along with related affordances, could also converge on a particular group of neurons thus providing a more general representation of "grasping." This would be the basis for generalized conceptual representations, which could be then related to language description (Aziz Zadeh and Damasio 2008).

These findings have been interpreted in terms of a mirror-neuron system, which automatically matches observed and executed actions (Rizzolatti and Craighero 2004). The secondary sensorimotor areas, which are known to be involved in mental simulation of world experiences, may be responsible for the representation of concepts (Gallese and Lakoff 2005). Instead, primary sensorimotor areas are presumed to be inhibited, in order to distinguish between a general representation of a given concept and its actualization.

This theory has been extended by Lakoff and his colleagues to include metaphors. Thus, the phrase "kick off the year" would also involve the motor representations related to kicking, just as the phrase "grasp the explanation" would involve motor representations related to control of the hand (Lakoff and Johnson 1999).
In a future prospective, the field of embodied semantics could be very fecund: understanding representations in the premotor cortex will be necessary to better understand how conceptual representations may arise. Furthermore, exploring the link between these conceptual representations and metaphorical language is especially important, as it has been proposed (Lakoff and Johnson 1999) that much of abstract thinking is performed metaphorically.

\section{Cross-References}

- Affordances

- Sensorimotor Schema

- Situated Cognition

\section{References}

Aziz Zadeh, L., \& Damasio, A. (2008). Embodied semantics for actions: Findings from functional brain imaging. Journal of Physiology, 102, 35-39.

Gallese, V., \& Lakoff, G. (2005). The brains concepts: The role of the sensory-motor system in reason and language. Cognitive Neuropsychology, 22, 455-479.

Garbarini, F., \& Adenzato, M. (2004). At the root of embodied cognition: Cognitive science meets neurophysiology. Brain and Cognition, 56, 100-106.

Lakoff, G., \& Johnson, M. (1999). Philosophy in the flesh. The embodied mind and its challenge to western thought. New York: Basic Books.

Rizzolatti, G., \& Craighero, L. (2004). The mirror-neuron system. Annual Review of Neuroscience, 27, 169-192.

Varela, F. J., Thompson, E., \& Rosch, E. (1991). The embodied mind. Cognitive science and human experience. Boston: MIT Press.

\section{Embodied Conversational Agents}

\author{
- Virtual Change Agents
}

\section{Embodied Embedded Cognition}

Embodied Cognition

\section{Embodied Learning}

- Phenomenology of Learning 


\section{Embodied Mind}

Embodied Cognition

\section{Embodiment}

Embodied Cognition

\section{Emergence of Learning Science}

History of the Sciences of Learning

\section{Emergent Learning}

A Salience Theory of Learning

\section{Emergentism}

Meaning Development in Child Language: A Constructivist Approach

\section{Emotion}

An emotion can be elicited consciously or unconsciously by evaluating a stimulus. Emotion is about positive and negative feelings, if it is positive a goal is advanced, if it is negative not. Emotion is a matter of cues for cognition and readiness to act. Emotions are experienced as distinctive types of mental states often accompanied by bodily changes/expressions (facial expression, posture, resulting activity/behavior, muscle action).

\section{Cross-References}

- Affective and Emotional Dispositions of/for Learning

- Arousal and Paired-Associate Learning

- Socio-emotional Aspects of Learning

\section{Emotion and Memory Interaction}

Emotional Memory

\section{Emotion Regulation}

Reinhard Pekrun

Department of Psychology, University of Munich, Munich, Germany

\section{Definition}

Emotion regulation refers to behaviors that aim to induce, modulate, or prevent emotions. Coping refers to the regulation of negative emotions and to the regulation of stress situations that cause these emotions (Zeidner and Endler 1996). In situations of learning, positive emotions can facilitate success, whereas negative emotions can be deleterious for learning and students' educational careers. By implication, emotion regulation in learning situations generally (but not always) implies strengthening or maintaining positive emotions and decreasing or preventing negative emotions.

\section{Theoretical Background}

Research on emotion regulation was strongly influenced by R. S. Lazarus' transactional model of stress and coping (Lazarus and Folkman 1984). In this model, stress is defined as any situation that taxes or exceeds one's capabilities, thus implying a threat to well-being and goal attainment. Purportedly, threat is cognitively evaluated in two appraisals. In a primary appraisal, the individual evaluates the likelihood and subjective importance of impending negative events. In a secondary appraisal, the individual explores possibilities to cope with the stress situation. To illustrate, a student about to take an important exam may make a primary appraisal about the likelihood of failure on the exam and a secondary appraisal about the possibilities of preventing failure. The student is expected to experience anxiety when the primary appraisal indicates that failure is likely, and when the secondary appraisal indicates that this threat is not sufficiently 
controllable. Subsequently, the individual can attempt to cope with the stressful situation and the resulting negative emotions. For instance, the student could employ strategies aimed toward changing the situation, changing the negative emotions, or ignoring the situation altogether. Such coping behaviors are thought to reciprocally influence the stressful situation and the resulting emotions, thus acting as mediators between stress and adaptive outcomes.

Recent research on emotion regulation has broadened this perspective to address both negative and positive emotions and a variety of regulatory strategies (e.g., John and Gross 2004; Ochsner and Gross 2008). Regarding emotions in learning and achievement more specifically, Pekrun's (2006) control-value theory of achievement emotions posits that emotions influence students' motivation and learning, and that learning outcomes reciprocally influence these emotions, their appraisal antecedents, and the social environments causing them. As such, achievement emotions, their antecedents, and their effects are thought to be linked by reciprocal causation over time. By implication, achievement emotions may be regulated by addressing any of the elements involved in these cyclic feedback processes. To this end, the individual could manage his emotions by targeting symptoms of the emotion itself (emotion-oriented regulation); by changing underlying appraisals (appraisal-oriented regulation); by changing learning environments (selection and design of environments); or by acquiring competences and studying more efficiently, thus making it possible to experience the emotional benefits of ensuing academic success (problem-oriented regulation).

\section{Important Scientific Research and Open Questions}

To date, little is known about emotion regulation in the context of learning and achievement. The only major exception is research on coping with test anxiety. Most of this research focused on (1) problem-oriented coping, (2) emotion-oriented and appraisal-oriented coping, and (3) avoidant coping (Zeidner 1998; Zeidner and Endler 1996; for a critical view, see Skinner et al. 2003). In contrast, the importance of selecting and changing learning environments and tasks as a means of regulating emotions at learning has been neglected.
With regard to learning and taking tests and exams, problem-oriented coping involves investing effort and employing strategies related to studying and performing tasks. Problem-oriented coping can have adverse effects when applied to tests and exams, such as increased situational anxiety, since dealing with the exam material can arouse thoughts about the upcoming exam (Bolger 1990). Over the long run, however, for most students the benefits associated with preparing and improving one's competences, such as increased academic performance and decreased anxiety, should outweigh negative situational effects (e.g. Zeidner 1995). Problem-oriented coping can be used to deal with emotions other than anxiety as well. Sansone et al. (1992) have shown that students can employ strategies of modifying tasks to make them more interesting, thus presumably alleviating task-induced boredom.

Emotion-oriented and appraisal-oriented coping aim to directly change unpleasant emotions. Typical strategies include (a) anxiety reduction by means of alcohol, nicotine, or pharmaceutical consumption, or by means of relaxation techniques; (b) reduction of emotional tension by simply accepting anxiety and the possibility of failure ("secondary control"; Morling and Evered 2006); (c) induction of positive, anxiety-incompatible emotions (e.g., by using humor, music, or emotional support from others); and (d) cognitively reappraising the situation as more controllable or less important. Many of these strategies are effective at reducing anxiety. However, some of them have negative side effects in terms of reduced achievement or health.

Avoidance-oriented coping implies behaviorally or mentally escaping from a stress-inducing situation. Examples of such strategies include (a) searching for mental distraction by focusing attention on taskirrelevant contents; (b) procrastination, prolonged phases of recess, and premature termination of studying; and (c) truancy, exam-avoidance, and dropout. Like emotion-oriented coping, these strategies can lead to a reduction of immediate anxiety; however, the side effects can be severe. First, consciously avoiding the experience of anxiety can lead to a detrimental increase of less conscious emotional arousal on a physiological level (Spangler et al. 2002). Moreover, although these strategies may temporarily reduce negative emotions, the underlying factors 
contributing to the experience of these emotions (e.g., low perceived control) go untreated. Finally, all of these strategies can be detrimental for students' learning and future career prospects.

Most of the literature on the regulation of test anxiety (and other negative emotions) used concepts of problem-oriented, emotion-oriented, and avoidance-oriented coping to classify strategies. However, specific strategies can often be classified into more than one category. For example, with regard to stress reduction, relaxation techniques imply emotion-oriented coping, but to the extent that tension reduction helps learning, they can also be regarded as problem-oriented coping. A related problem is the common assumption that problem-oriented coping should be adaptive, and that emotion-oriented or avoidance-oriented coping should be maladaptive since neither changes the stressinducing situation. However, different criteria can be used to judge adaptation (e.g., is it more important to increase achievement or to live a life free of excessive anxiety?). Furthermore, any strategy can have secondary effects that also can be either adaptive or maladaptive. For example, while time-consuming academic studying can raise learning outcomes, it can also cause friendships to dissolve, implying that problem-oriented coping need not always be beneficial.

Future research on emotion regulation in learning and achievement should make use of current conceptions of regulation by addressing a broader variety of relevant emotions, including anxiety as well as emotions such as enjoyment, pride, anger, frustration, shame, hopelessness, or boredom. Also, research should address the multiple antecedents and outcomes of regulatory behavior, including methods learners can use to self-select and modify environments and tasks for learning. Moreover, research should explore possibilities to design learning environments and educational intervention programs such that learners become enabled to self-regulate their emotions during learning.

\section{Cross-References}

- Affective and Cognitive Learning in the Online Classroom

- Affective and Motivational Dispositions of/for Learning

- Affective Dimensions of Learning

- Affective Priming and Learning
- Emotional Intelligence and Learning

- Emotional Schema(s)

- Emotion-Based Learning

- Emotions and Learning

\section{References}

Bolger, N. (1990). Coping as a personality process: A prospective study. Journal of Personality and Social Psychology, 59, 525-537.

John, O. P., \& Gross, J. J. (2004). Healthy and unhealthy emotion regulation: Personality processes, individual differences, and life span development. Journal of Personality, 72, 1302-1333.

Lazarus, R. S., \& Folkman, S. (1984). Stress, appraisal, and coping. New York: Springer.

Morling, B., \& Evered, S. (2006). Secondary control reviewed and defined. Psychological Bulletin, 13, 269-296.

Ochsner, K. N., \& Gross, J. J. (2008). Cognitive emotion regulation. Insights from social cognitive and affective neuroscience. Current Directions in Psychological Science, 17, 153-158.

Pekrun, R. (2006). The control-value theory of achievement emotions: Assumptions, corollaries, and implications for educational research and practice. Educational Psychology Review, 18, 315-341.

Sansone, C., Weir, C., Harpster, L., \& Morgan, C. (1992). Once a boring task always a boring task? Interest as a self-regulatory mechanism. Journal of Personality and Social Psychology, 63, 379-390.

Skinner, E. A., Edge, K., Altman, J., \& Sherwood, H. (2003). Searching for the structure of coping: A review and critique of category systems for classifying ways of coping. Psychological Bulletin, 129, 216-269.

Spangler, G., Pekrun, R., Kramer, K., \& Hofmann, H. (2002). Students' emotions, physiological reactions, and coping in academic exams. Anxiety, Stress, and Coping, 15, 413-432.

Zeidner, M. (1995). Coping with examination stress: Resources, strategies, outcomes. Anxiety, Stress, and Coping, 8, 279-298.

Zeidner, M. (1998). Test anxiety: the state of the art. New York: Plenum.

Zeidner, M., \& Endler, N. (Eds.). (1996). Handbook of coping: Theory, research, applications. New York: Wiley.

\section{Emotion scheme}

- Emotional Mental Models

\section{Emotional and Cognitive Learning}

- Visual Communication and Learning 


\section{Emotional Aspects in Learning}

Affective Dimensions of Learning

\section{Emotional Body Gesture Learning}

Learning Human Emotion from Body Gesture

\section{Emotional Conceptual Model}

Emotional Mental Models

\section{Emotional Dimensions of Learning}

- Affective Dimensions of Learning

- Emotional Learning

\section{Emotional Expression Development}

Facial Expression Learning

\section{Emotional Factors in Learning}

Affective Dimensions of Learning

- Emotional Learning

\section{Emotional Intelligence}

- Emotional Learning

- Social-Emotional Learning Scale

\section{Emotional Intelligence and Learning}

\author{
H. Harald Freudenthaler ${ }^{1}$, K. V. Petrides ${ }^{2}$ \\ ${ }^{1}$ Department of Psychology, University of Graz, \\ Graz, Austria \\ ${ }^{2}$ Department of Psychology, University College \\ London (UCL), London, UK
}

\section{Definitions}

Ability emotional intelligence: A speculative construct positing the existence of quantifiable cognitive differences in the ways in which typically developed individuals attend to, experience, process, and utilize emotions. The fundamental problem with ability EI is the inherently subjective nature of emotion, which undermines objective measurement using veridical scoring criteria (as in IQ tests).

Operationalization: The process of deriving an operational definition for a construct. Operational definitions are predicated on the directly measurable operations that produced the construct being defined. For example, pulse is defined as the number of heartbeats per minute.

Trait emotional intelligence: A constellation of emotional self-perceptions located at the lower levels of personality hierarchies. The construct essentially concerns people's self-perceptions of their emotional abilities.

Veridical scoring criterion: A logically objective basis for determining whether a response to a test item or task is correct or incorrect.

\section{Theoretical Background}

\section{Trait El Versus Ability El}

Two constructs of emotional intelligence (EI) are distinguished based on the measurement method used in their operationalization process (self-report, as in personality questionnaires, or maximum-performance, as in IQ tests; Petrides et al. 2007). Trait EI (or "trait emotional self-efficacy") concerns emotional selfperceptions measured via self-report, while ability EI (or "cognitive-emotional ability") concerns cognitiveemotional abilities that, in theory, should be measured via maximum-performance tests. The conceptual 
differences between the two constructs are directly reflected in empirical findings, which reveal low, often nonsignificant, correlations between measures of trait and ability EI, thereby supporting an explicit distinction between the two.

Theoretically, trait and ability EI can "coexist" because the operationalization of one does not preclude the operationalization of the other. However, a string of publications by psychometricians and emotion theorists alike have cast doubt on the validity of ability EI. Therefore, it seems increasingly probable that this construct, like other faux intelligences (social, spiritual, practical, etc.), will be left out of the mainstream taxonomies of human cognitive ability.

\section{Definition and Measurement of Ability Emotionall Intelligence}

The ability approach conceives EI as a form of intelligence and separates it from (emotion-related) personality attributes by emphasizing individual differences in reasoning about emotions and in the processing of affective information to enhance thinking (Mayer et al. 2004). Ability EI comprises four interrelated branches arranged hierarchically from the most basic (perception of emotion, involving receiving and recognizing emotional information) to the more developmentally complex, including emotional facilitation of thought (the capacity of emotions to assist thinking), understanding of emotion (the capacity to analyze emotions and understand their outcomes) and management of emotion (the capacity to adaptively regulate moods and emotions in oneself and others).

Several putatively objective "maximum-performance" (MP) measures of EI have been constructed. For example, the Mayer-Salovey-Caruso Emotional Intelligence Test (MSCEIT) comprises eight subtests (two for each branch) for the measurement of the four-branch model of EI. The highest branch (managing emotions), which encompasses the most advanced skills, is assessed by two scenario-based subtests requiring subjects to determine how effective several different courses of action would be in managing their own emotions (five scenarios; four responses each) and the emotions of others (three scenarios; three responses each). Test scores are derived via two methods: consensus and expert scoring.

\section{Ability Emotional Intelligence and Learning}

Ability EI is thought to influence success at school, including learning, peer relationships, and school performance (Brackett and Katulak 2006). For instance, students' ability to manage their own emotions - which are known to drive attention, motivation, and memory - may facilitate learning via enhanced concentration or motivation. Emotion management skills may also help students handle stressful situations, such as taking tests (e.g., by being able to stay calm before and during exams in order to perform well). Moreover, emotionrelated management skills are thought to facilitate the development of quality social relationships (including better support from peers and teachers) and healthier attachments to school (feeling more comfortable, secure, and valued in the school environment), thus further enhancing motivation.

\section{Important Scientific Research and Open Questions}

Research provides some evidence for the proposed associations of ability EI with academic and social success. MP measures of EI have been associated with higher grades, better relationships, fewer negative interactions, and less conflict with peers and friends, as well as with lower levels of deviant or maladaptive behavior, including fighting, drug use, or alcohol consumption (Brackett and Katulak 2006; Mayer et al. 2004). However, the incremental validity of ability EI in predicting school performance is weak. The vast majority of the obtained correlations between ability EI and grades tend to drop to non-significance once cognitive intelligence is partialled out.

At first glance, the negative findings concerning incremental validity undermine the relevance of emotion-related skills for academic success. However, it has to be taken into account that MP measures of EI, among other problems, are limited in their ability to measure emotional management. Freudenthaler, Neubauer, and Haller (2008) demonstrated that MP measures of intraand interpersonal emotion management merely assess individuals' declarative knowledge about the effectiveness of various behaviors in emotional situations. This cannot be equated with actual behavioral effectiveness typically shown in such situations.

The interrcorrelations between knowledge-related MP measures and behavioral measures of intrapersonal 
$(r=.04)$ and interpersonal $(r=.34, p<.01)$ emotion management further emphasize that the presumed translation of emotional knowledge into practice, as suggested by the ability EI approach, is largely restricted to interpersonal skills. It seems clear that managing emotions in oneself effectively, thought to contribute to academic adaptation, requires more than the availability of emotional knowledge. Accordingly, it is not surprising that ability EI measures fail to predict school achievement over and above cognitive intelligence in most studies or that training programs designed to teach emotional knowledge in order to change behavior fail to attain desired outcomes (Mayer et al. 2004). Emotional and social learning programs should not be exclusively focused on improving students' socioemotional knowledge; they also have to be aimed at developing their expertise to employ this knowledge appropriately as well as their motivation and willingness to put expertise into practice.

\section{Definition and Measurement of Trait El}

Trait EI is defined as a constellation of emotional selfperceptions located at the lower levels of personality hierarchies (Petrides et al. 2007). The label of the construct reflects the fact that the various notions that have been discussed in the literature under the terms "emotional intelligence" or "EQ" (Goleman 1995) invariably and mainly describe personality traits. The content domain of the construct comprises the affective aspects of personality (see Table 1 for the constituent facets). In lay terms, trait EI concerns people's selfperceptions of their emotional abilities. Two important advantages of trait EI theory is that it takes into account the subjective nature of emotional experience and that it integrates the construct into mainstream scientific models of differential psychology. Further, trait EI theory is testable, falsifiable, and general in the sense that it is not tied to proprietary measures, but can provide a basis for the interpretation of findings from any questionnaire of EI or related constructs.

There exist many questionnaires whose authors erroneously claim they measure EI as intelligence. It has been pointed out that such questionnaires operationalize a construct that is unrelated to capabilities, competencies, and skills. Rather, they provide coverage, of variable quality and adequacy, of the sampling domain of trait EI as shown in Table 1. They can (and should), therefore, be seen as measures of trait EI in order for their results to be meaningfully interpreted.

Emotional Intelligence and Learning. Table 1 The sampling domain of trait El in adults

\begin{tabular}{|c|c|}
\hline Facets & High scorers view themselves as. \\
\hline Adaptability & .. flexible and willing to adapt to new conditions \\
\hline Assertiveness & ... forthright, frank, and willing to stand up for their rights \\
\hline Emotion expression & ... capable of communicating their feelings to others \\
\hline Emotion management (others) & . . capable of influencing other people's feelings \\
\hline Emotion perception (self and others) & ... clear about their own and other people's feelings \\
\hline Emotion regulation & . . capable of controlling their emotions \\
\hline Impulsiveness (low) & ... reflective and less likely to give in to their urges \\
\hline Relationships & ... capable of maintaining fulfilling personal relationships \\
\hline Self-esteem & ... successful and self-confident \\
\hline Self-motivation & ... driven and unlikely to give up in the face of adversity \\
\hline Social awareness & . . accomplished networkers with superior social skills \\
\hline Stress management & .. capable of withstanding pressure and regulating stress \\
\hline Trait empathy & ...capable of taking someone else's perspective \\
\hline Trait happiness & ...cheerful and satisfied with their lives \\
\hline Trait optimism & ...confident and likely to "look on the bright side" of life \\
\hline
\end{tabular}


It is important to remember, however, that EI-related questionnaires can be considered measures of trait EI only in so far as their results are interpreted through the lens of trait EI theory. The use of any EI questionnaire that is subsequently interpreted through the poppsychology perspective of "EQ is good for you" renders the instrument ineffective as an operationalization vehicle for trait EI theory.

\section{Trait Emotional Intelligence and Learning}

The absence of strong correlations between personality and cognitive ability does not preclude the possibility of simultaneous effects on criteria like academic performance. Even though trait EI is conceptualized as orthogonal (i.e., unrelated) to cognitive ability, it is quite possible that it can have indirect, and even small direct, effects on academic achievement. Below, we summarize the main findings of the literature on trait EI and academic achievement at both the primary and secondary levels.

\section{Primary School}

Much trait EI research in children has been conducted with measures that are mere adaptations of the respective adult forms, which means that they are based on content domains that are inappropriate for children. Mavroveli, Petrides, Shove, and Whitehead (2008) derived a sampling domain specifically for children aged 8-12 years and devised an inventory to provide comprehensive measurement of this domain. In line with trait EI theory, they found that the construct was only weakly, if at all, related to academic achievement. The results suggested that possible confounding with verbal intelligence may lead to low or moderate correlations with proxies of academic achievement, especially in heterogeneous samples or samples of young children. Related findings showed that trait EI can predict unauthorized absences (truancy) and exclusions due to breaches of discipline, thus differentiating between well- and poorly-adjusted children at school.

\section{Secondary School}

It has been shown that IQ moderates the relationship between trait EI and achievement, such that high trait $\mathrm{EI}$ is associated with better academic performance in low IQ adolescents only. In other words, the association may not be present in adolescents with average or high IQ scores. This suggests that such effects as trait EI might have on performance are likely to assume prominence when the demands of a situation outweigh an adolescent's intellectual resources. Modest direct correlations $(r=0.20, p<0.05)$ between trait EI and academic performance in high school and university samples have also been reported, raising the possibility that the effects of trait EI may vary across educational levels as well as across subjects, like those of other personality traits (e.g., agreeableness). As in primary school, it has been found that adolescents with high trait EI have fewer unauthorized absences and are less likely to have been excluded from class due to breaches of discipline. Thus far, the picture emerging is consistent with the trait EI hypothesis that the construct's direct impact on academic achievement is modest and likely to be more relevant to specific groups of vulnerable children (e.g., those with low cognitive ability or learning disabilities).

\section{Cross-References}

- Ability-Based Learning

- Affective and Emotional Dispositions of/for Learning

- Cognitive and Affective Learning Strategies

- Emotional Learning

- Emotional Regulation

- Multiple Intelligences and Learning Styles

- Personality and Learning

- Personality Effects on Learning

- Self-efficacy for Self-regulated Learning

- Self-esteem and Learning

\section{References}

Brackett, M. A., \& Katulak, N. A. (2006). Emotional intelligence in the classroom: Skill-based training for teachers and students. In J. Ciarrochi \& J. D. Mayer (Eds.), Improving emotional intelligence: A practitioners guide (pp. 1-27). New York: Psychology Press/Taylor \& Francis.

Freudenthaler, H. H., Neubauer, A. C., \& Haller, U. (2008). Emotional intelligence: Instruction effects and sex differences in emotional management abilities. Journal of Individual Differences, 29, $105-115$.

Goleman, D. (1995). Emotional intelligence: Why it can matter more than IQ. London: Bloomsbury.

Mavroveli, S., Petrides, K. V., Shove, C., \& Whitehead, A. (2008). Investigation of the construct of trait emotional intelligence in children. European Child Adolescent Psychiatry, 17, 516-526. 
Mayer, J. D., Salovey, P., \& Caruso, D. (2004). Emotional intelligence: Theory, findings, and implications. Psychological Inquiry, 15, 197-215.

Petrides, K. V., Pita, R., \& Kokkinaki, F. (2007). The location of trait emotional intelligence in personality factor space. British Journal of Psychology, 98, 273-289.

\section{Emotional Intelligence in Animals}

\section{MARC BeKoff}

Ecology and Environmental Biology, University of Colorado, Boulder, CO, USA

\section{Synonyms}

Feelings; Mood; Passions; Temperament

\section{Definition}

The word "emotion" has been a difficult one to define, but available definitions generally view an emotion as a mental state that is accompanied by physiological changes that may or may not lead to various actions including fighting, fleeing, seeking a specific activity including play, safety, or comfort with another individual, or moving to a specific location where an individual feels secure. Emotions can be instinctual or spontaneous, or have a cognitive or conscious component.

\section{Theoretical Background}

Interest and research on animal emotions has a long history dating back to early Greek philosophers and naturalists. Charles Darwin was keenly interested in animal emotions and wrote about them in his classic books On the Origin of Species (1859), The Descent of Man (1871), and The Expression of the Emotions in Man and Animals (1872).

\section{Important Scientific Research and Open Questions}

Numerous lines of independent evidence from many different disciplines show that many animals have rich and deep emotional lives (Balcombe 2006; Bekoff 2000, 2007, 2010; Panksepp 2005). When taken as a whole, the ensemble of data leads to no other reasonable conclusion. We know that dogs, cats, and numerous other animals like to play, feel joy as they run here and there, and get lonely and sad. We know that elephants do not like having their families broken up and that pigs do not like having their tails cut off (docked) or their teeth ground down on grinding stones without anesthesia. And, coyotes don't like being trapped, wolves don't like being chased and shot, nor do chimpanzees or other animals enjoy spending their lives in small impoverished cages where they can barely stretch or move.

What is exciting about studying the emotional lives of animals, their pains, passions and virtues, what they like and do not like, is that all sorts of "big questions" are raised. By simply asking what a dog feels as she plays with her friends, or what an elephant feels when he has lost his friends or is caged alone in a zoo, we confront questions about empathy, theory of mind, self-cognizance, anthropomorphism, and the role that scientific data, evolutionary biology, and common sense and intuition play in our coming to a better understanding and appreciation of the emotional lives of animals.

It is no longer a question if certain animals (let us focus on mammals for the moment) have rich and deep emotional lives, it is a matter of why they have evolved and what are they good for. We no longer have to put quotation marks that reflect our uncertainty around words such as "happy" or "sad" when we say that Fido, a dog, is happy or sad. He is just happy or sad in his own ways just as we are happy or sad in our own ways. Emotions have evolved because they function as "social glue," playing major roles in the formation and maintenance of social relationships among animals. Emotions also serve as "social catalysts," emoting individuals to follow different courses of action depending on the situation in which they find themselves. Charles Darwin's ideas about evolutionary continuity, that differences among species are differences in degree rather than differences in kind, come into play in the study of emotions as they do in investigations of comparative anatomy and physiology. The bottom line to ideas about evolutionary continuity is that "if we have something, in this case emotional intelligence, they (other animals) do too."

Animals' lives are rather public. While there might not always be full disclosure and there will always be mysteries, there is much transparency. If we carefully study animal behavior we can get a strong grasp on what animals are experiencing and feeling. What is 
especially and exciting and important is that noninvasive neural imaging techniques such as functional magnetic resonance imaging (fMRI) and positron emission tomography (PET) scans are allowing researchers to localize brain activity when animals are in specific social situations.

We also need time to assess the accuracy of our intuitions about emotional intelligence in animals. Indeed, there are studies that show that there is a lot of agreement among people concerning what animals are feeling and these intuitions are quite good at predicting what an individual is likely to do in the future. For example, Wemelsfelder and her colleagues (2000) discovered that even people who have little experience observing animals usually agree with one another on what an animal is most likely feeling. Their intuitions are borne out because their characterizations of animal emotional states predict future behavior quite accurately. Highly accurate prediction offers one of the strongest arguments for the existence of emotions and feelings in nonhuman species.

In another study, Wemelsfelder and her colleagues asked 18 naïve observers to independently and describe in their own words the behavioral expression of 20 pigs. They discovered that the observers showed "significant agreement in their spontaneous assessment of pig expressed, which suggests that these assessments were based on commonly perceived and systematically applied criteria." Wemelsfelder's and her colleagues' extensive research shows that much of an animal's subjective experience of suffering or well-being is public, not hidden or private. What animals do expresses their personal and subjective experiences.

That many animals have subjective and intersubjective communal lives - other animals are in their thoughts and feelings - and a personal point of view on the world that they share with other individuals, seems beyond question. Bekoff and Pierce (2009) also posit that animals have moral intelligence.

It is important to study animals where they are able to express a full range of behavior and have normal social interactions, and not rely heavily on controlled studies in impoverished environments, the nature of which often preclude getting reliable answers to the questions of interest because animals are bored, stressed, or kept in unnatural social groups in small cages. Since the main question is why emotions have evolved and why it is important to understand them, an agenda is set for an exciting journey into the hearts and minds of other animals.

\section{Cross-References}

- Animal Learning and Intelligence

- Emotional Intelligence and Learning

- Emotional Regulation

- Emotions: Functions, Evidences, and Effects on Learning

- Neuropsychology of Emotion

- Play, Exploration, and Learning

\section{References}

Balcombe, J. (2006). Pleasurable kingdom: Animals and the nature of feeling good. London: Macmillan.

Bekoff, M. (Ed.). (2000). The smile of a dolphin: Remarkable accounts of animal emotions. Washington, DC: Random House/Discovery Books.

Bekoff, M. (2007). The emotional lives of animals. Novato: New World Library.

Bekoff, M., \& Pierce, J. (2009). Wild justice: The moral lives of animals. Chicago: University of Chicago Press.

Bekoff, M. (2010). The animal manifesto: Six reasons for expanding our compassion footprint. Novato: New World Library.

Panksepp, J. (2005). Beyond a joke: From animal laughter to human joy. Science, 308, 62-63.

Wemeslfelder, F., Hunter, E. A., Mendl, M. T., \& Lawrence, A. B. (2000). The spontaneous qualitative assessment of behavioural expressions in pigs: First explorations of a novel methodology for integrative animal welfare measurement. Applied Animal Behaviour Science, 67, 193-215.

\section{Emotional Learning}

Claude Frasson $^{1}$, Alicia Heraz ${ }^{2}$

${ }^{1}$ Department of Computer Science, University of

Montreal, Montréal, Québec, Canada

${ }^{2}$ University of Montreal, Montréal, Québec, Canada

\section{Synonyms}

Affective learning; Emotional dimensions of learning; Emotional factors in learning; Emotional intelligence; Learning by feeling; Social learning

\section{Definition}

Emotional learning (EL) is related to one of the three domains identified in Bloom's taxonomy of learning (Bloom 1956). Bloom identified, cognitive, affective, 
and psychomotor as the three domains of educational objectives. The cognitive domain is described by Bloom as, "the recall or recognition of knowledge and the development of intellectual abilities and skills." The affective domain is described as, "objectives which describe changes in interest, attitudes, and values, and the development of appreciations and adequate adjustment." Lastly, the psychomotor domain refers to the motor-skills or behavioral skills that constitute the relationship between cognitive process and the physical movement in education (San Jose and Kelleher 2009). The term Emotional learning has been defined as "an increasing internalization of positive attitudes toward the content or subject matter" (Scott and Wheeless 1975). Stancato and Hamachek (1990) defined it as "the attitudes and feelings that students have about themselves."

Emotional Learning is associated to two fundamental concepts: Emotional Intelligence (EI) and Social and Emotional Learning (SEL). Emotional Intelligence is the capacity or ability for somebody to identify, assess, and control his emotions or the emotions of others. Social and Emotional Learning consists in learning how to manage feelings and relationships with others. This includes ways to also recognize emotions and to maintain positive relationships in developing sympathy and empathy.

Many researchers of Human Behavior and Learning have helped to evolve the meaning of emotion from a synonym for affect to a deeper understanding that while emotion is an affective state, not all affective states are emotions (McLeod 1997). There is little agreement on how to define the term (Stricker 2009); the word became associated across a wide range of contradictory concepts and phenomena involving affect and feelings (Owens and Maxmen 1979).

\section{Theoretical Background}

The main elements of an EI model were set by Salovey and Mayer (1990), then by Goleman (1995). The model is based on four abilities: (1) Perceiving emotions: how to detect and understand emotions through images of faces, voices, pictures, and cultural elements. (2) Using emotions: how to use emotions for facilitating cognitive activities, thinking, and problem solving. (3) Understanding emotions: how to understand variations of emotions. (4) Managing emotions: how to regulate emotions and exploit emotions to achieve intended goals.

Goleman outlined five emotional skills for SEL: (1) Self and other awareness: understanding and recognizing feelings and their changes, the impact of actions on feelings, and the difference between thinking, feeling and acting. (2) Mood management: controlling strong variations of feelings of anger. (3) Self-motivation: continuing positively the actions toward established goals. (4) Empathy: taking care of someone's emotions and cognitions. (5) Management of relationships: resolving conflicts, establishing friendships, and collaborative learning.

In the early 1960s, Piaget (1962) predicted the important role of emotion in learning: "At no level, at no state, even in the adult, can we find behavior or a state that is purely cognitive without emotion nor a purely emotional state without a cognitive element involved." In the late twentieth century, affect grew in importance with investigations on the ways people acquire, interpret, shape, and sharpen information via thinking and affective skills.

Many researchers have explored the link between cognition and emotional dispositions where humans approached thinking and learning. Some of the results show strong connections according to specific contexts. The main insights emerged from social cognitive theory research on the role played by several dispositions across creative, critical, and self-regulated thinking. The dispositions explored as synthesized by Stricker (2009) are listed below:

1. Seeking clarity and precision when information is unclear

2. Trying to be well informed

3. Seeking reasons for what you believe

4. Taking into account the total situation

5. Carefully analyzing information

6. Remaining open-minded

7. Taking a position (and changing it) when the evidence is sufficient to do so

8. Showing sensitivity to the feelings, level of knowledge, and degree of sophistication of others

9. Resisting impulsivity

10. Engaging intensely in tasks even when answers or solutions are not immediately apparent

11. Pushing the limits of one's knowledge and abilities to keep improving on one's knowledge and skills 
12. Generating, trusting, and maintaining one's own standards of evaluation

13. Generating new ways of viewing a situation outside the boundaries of standard conventions

14. Planning

15. Being sensitive to feedback

16. Evaluating progress

17. Making use of available resources

In certain contexts, humans can form mental positions about learning and associate it to a strong emotion. They reflect a mental attitude for evaluations of good, bad, or neutral. Mental attitudes can play an important role in how a human views, engages, and values a learning context.

From a physiological point of view, the amygdala is involved with the creation and storage of emotional reactions and events. When, for instance, fear or anxiety happen they send signals to the amygdala which stores the event into memory associated with the anxiety state. A similar event will raise similar emotional states. Memorizing emotions will maintain typical synaptic connexions triggering symptoms of anxiety or fear if the event occurs again.

There is a strong relationship between emotion and social knowledge structures. Several research works show that knowledge is essentially situated; it depends on the context in which it is actively constructed and reconstructed through interactions, time of reflection, and the process of interpretation (Rogoff and Lave 1984). Knowledge is also dependent on "the point of observation" of the human which simultaneously shapes and is shaped by social reality (Stricker 2009).

Deeper understanding about the way knowledge is embedded in context and the media allowing its expression has been explored by many researchers on human learning (Bransford et al. 2000). There is a deep connection between humans and technology via the use of personal and cultural tools now supporting human minds, senses, and bodies (D'Mello et al. 2009; Heraz and Frasson 2008).

Moreover, there is growing research interest on the symbiotic connection between human minds and digital tools making possible phenomenal capabilities via interconnected and distributed ways of knowing and learning. For example, new media digital technologies can affect learning in several fundamental ways. Electronic texts can have hypertext providing for multidimensional and less linear-oriented interaction between the learner and content (Stricker 2009).

Programs of SEL have been set up in some universities or institutes to enhance students' self-awareness, social awareness, self-regulation, collaborative behaviors, together with positive attitudes and emotional states such as happiness, optimism, and altruism. The goal is, through a series of exercises and practices, to calm students' minds, concentrate their attention, and make them more aware of their environment and others.

\section{Important Scientific Research and Open Questions}

As emotional learning is based on emotions, one of the important open questions remains on the difficulty to identify precisely the emotional state of the learner. Different methods such as face recognition, questionnaires, and detection from physiological sensors are used and can be combined. They still lack relevance. What is the intensity of the emotion expressed by a smile? How to assess or estimate the intensity of emotions? What is the duration of an emotion? The transition from an emotional state to another depends on various factors and first of the learner. The duration of moods depends on several parameters such as the personality of the learner, the chemical state of the brain, and the influence of emotional intensity of events. Induction of emotions to improve learning has to be quantified in order to be controlled. Several investigations need to be undertaken in this area.

In order to advance education, intelligent learning environments and human-computer interfaces by optimally coordinating cognition and emotions, considerable research has been undertaken, but it has not completely investigated students' emotional processes or affective learning (Woolf et al. 2007).

New pedagogical strategies based on emotion management and control need to be used in teaching and learning. The value-added programs of EL and SEL in knowledge acquisition imply to investigate, develop, and integrate emotional strategies to complete and enhance the cognitive aspects of actual educational programs. Effective assessment and reliable data will be essential to create structures and conditions for SEL.

In particular, social learning requires creating social structures and programs to improve understanding of 
SEL components. Integration of these programs into the educational system is a first challenge. Measurement of the positive effects on the students remains a second challenge.

Impact of social learning is effectively difficult to establish as it concerns various parameters to consider: improvement of knowledge acquisition, of behavior, relationships with the environments, cooperation, and conflict resolution. Time is necessary to gather enough pertinent data to assess the contribution of these approaches.

Finally a new track of research in emotional learning concerns advanced capabilities of the brain submitted to subliminal cues (Chalfoun and Frasson 2009).

\section{Cross-References}

- Affective Dimensions of Learning

Emotion-Based Learning

- Learning by Feeling

- Stress and Learning

\section{References}

Bloom, B. S. (1956). Taxonomy of educational objectives. In Handbook I: Cognitive domain. New York: David McKay.

Bransford, J., Brown, A., \& Cocking, R. (Eds.). (2000). How people learn: Brain, mind, experience, and school. Washington, D.C.: National Academy Press.

Chalfoun, P., \& Frasson, C. (2009). Optimal affective conditions for subconscious learning in $3 D$ intelligent tutoring system. Los Angeles: Human Computer Interaction.

D’Mello, S. K., \& Graesser, A. C. (2009). Automatic detection of learner's affect from gross body language. Applied Artificial Intelligence, 23, 123-150.

Goleman, D. P. (1995). Emotional intelligence: Why it can matter more than IQ for character, health and lifelong achievement. New York: Bantam Books.

Heraz, A., \& Frasson, C. (2008). Predicting the three major dimensions of the learner's emotions from brainwaves. International Journal of Computer Science, 2(3), 187-193.

McLeod, S. H. (1997). Notes on the heart: Affective issues in the writing classroom. Carbondale, IL: Southern Illinois University Press.

Owens, H., \& Maxmen, J. S. (1979). Moods and affect: A semantic confusion. The American Journal of Psychiatry, 136, 97-99.

Piaget, J. (1962). The relationship of affectivity to intelligence in the mental development of the child. Bulletin of the Menninger Clinic, 26, 129-137.

Rogoff, B., \& Lave, L. (1984). Everyday cognition: Its development in social context. Cambridge, MA: Harvard University Press.

Salovey, P., \& Mayer, J. D. (1990). Emotional intelligence. Imagination, Cognition, and Personality, 9, 185-211.

San Jose, D., \& Kelleher, T. (2009). Measuring ecoshock and affective learning: A comparison of student responses to online and face-to-face learning ecologies. Journal of Online Learning and Teaching, 5, 469-476.

Scott, M. D., \& Wheeless, L. R. (1975). Communication apprehension, student attitudes, and levels of satisfaction. Western Journal of Speech Communication, 41, 188-198.

Stancato, F. A., \& Hamachek, A. L. (1990). The interactive nature and reciprocal effects of cognitive and affective learning. Education, $111,77-81$.

Stricker, A. G., (2009). Why affective learning in a situated place matters for the millennial generation. A4/6I Innovations and Integration Division, Air University, 23 July 2009.

Woolf, B., Burleson, W., \& Arroyo, I. (2007). Emotional intelligence for computer tutors. Supplementary proceedings of the 13th international conference on artificial intelligence in education (AIED 2007), Los Angles, pp. 6-15.

\section{Emotional Memory}

\author{
Elizabeth A. Kensinger, Brendan D. Murray \\ Department of Psychology, Boston College, \\ Chestnut Hill, MA, USA
}

\section{Synonyms}

Affect and memory; Emotion and memory interaction; Flashbulb memory

\section{Definition}

"Emotional memory" is shorthand for denoting the memory of experiences that evoked an emotional reaction. It is most commonly used to refer to the ability to consciously remember aspects of those experiences; in other words, the term is used to describe the effects of emotion on episodic memory.

There is not a universally accepted definition for the term "emotion." Although everyone has a subjective understanding of what it means to have an emotional response to something, pinpointing the necessary and sufficient features for such a response has proven to be difficult. Most memory researchers have considered an event to be "emotional" in nature if a person perceives the event to be pleasant or unpleasant in nature (to have positive or negative valence associated with it) or if the event elicits a change in the person's arousal level, altering how calm or how excited they are. These dimensions of valence and arousal are presumed to be orthogonal to one another, forming a circumplex of emotion described by James Russell. 


\section{Theoretical Background}

Anecdotally, most of us believe that we remember events that have some emotional context better than those that do not. We may not remember the details of biking to work most days last month, but we probably do remember the bike ride during which we were chased by an angry dog. This fact that we would selectively remember emotional experiences makes sense as an adaptive feature of human memory that could help us to prioritize those things that are important to us (e.g., remembering to take a different route when biking to work).

Roger Brown and James Kulik (1977) have called the most extreme form of such an emotional memory a "flashbulb memory," in which an emotional event is remembered so vividly as to be almost photographic in nature. Such memories might include memory for the New York City terrorist attacks of September 11th, the resignation of Prime Minister Margaret Thatcher, or your wedding day. What is remarkable about these "flashbulb memories" is not that participants can remember that the event occurred but that they can remember many details of the personal context in which they experienced the event; people might remember what they were wearing, who they were with, or what they were doing when they learned the news. One related debate that has arisen is whether or not there is some "special" mechanism underlying emotional memory: does memory for emotional information require processes that are different from memory for neutral information? Or, do emotional memories recruit the same processes as neutral memories, but just recruit them differently? While this is still an open debate, there appears to be evidence that there are processes specific to memory for emotional information (for example, the amygdala likely plays a critical role in the formation of emotional memories, while it does not do so for more emotionally neutral memories).

Even when "flashbulb memories" do not form, emotion is still believed to affect each of the stages involved in the representation of an event in memory: at encoding, when the information is initially encountered and converted into some form that it can be stored in memory; at consolidation, when the information is stabilized in memory following encoding; or retrieval, when the information is accessed from memory.

\section{Encoding}

At encoding, emotionally salient information is typically prioritized over information that is nonemotional. This can be seen in the way we orient our attention: it has been shown repeatedly that people usually identify emotionally relevant aspects of a scene (such as a gun) before they attend to or identify other details (such as the face of the person holding the gun). The term "weapon focus effect" has been used to describe this phenomenon, describing how we may focus our attention on such emotionally relevant material (and often do so at the expense of other peripheral detail).

One demonstration of orienting attention to emotional material comes from Arne Öhman and colleagues (2001). They showed participants $3 \times 3$ matrices of pictures, in which either all nine pictures were different exemplars of the same category (e.g., nine different chairs), or eight were different exemplars of the same category and one different (e.g., eight different chairs and one airplane). When the discrepant item had an emotional content - for example, if it were a spider or snake - participants were substantially faster and locating the discrepant item than when it was nonemotional.

\section{Consolidation}

Emotional memories are also more likely to be consolidated into long-term memory than neutral memories are. This may be partly due to emotional information being prioritized at encoding, but it is likely that other mechanisms occur after initial encoding to ensure that emotional memories persist. In his review of memory consolidation in 2000, James McGaugh notes that emotional information may trigger certain stress hormones that promote long-term memory consolidation. Indeed, Tali Sharot and Elizabeth Phelps showed that if memory was tested for emotional and neutral items immediately after they were studied, there was no benefit for emotional items; however, if memory was probed after a delay, an emotional memory benefit was seen. This pattern of results would be consistent with the hypothesis that emotion is increasing the likelihood that emotional information is maintained over time: Although neutral information may decay over time, emotional information may remain in our memory stores. While the extant literature on emotion and consolidation 
processes is somewhat limited, there is mounting evidence to suggest that emotion does act on the consolidation phase of memory to promote long-term retention of emotional memories.

\section{Retrieval}

Emotional memories are also more likely to be retrieved than neutral memories. When individuals are asked to remember events from their own past, often referred to as autobiographical memory, they are often more likely to offer memories that were in some way emotionally important to them. People are also often more likely to retrieve memories that are congruent with their current mood: when we are angry or upset, we are more likely to retrieve memories for negative events, and when we are in a good mood we are more likely to retrieve memories for positive events.

\section{Semantic Networks for Emotional Information}

It has been suggested that another factor contributing to the salience of emotional memory is how semantically connected emotional information is to other information in memory. For example, Deborah Talmi and Morris Moscovitch (2004) gave people lists of words - both emotional and neutral - and asked them to rate how well each word was related to words in a different list. In general, people endorsed emotional items as having more "high associates" than neutral items to words in the second list. At any stage of memory, then, emotional memories may benefit from additional elaboration. Because emotional information may be easier to relate semantically to other information in memory, it may be easier for people to make personally relevant links between new and old information in memory.

\section{Important Scientific Research and Open Questions}

Many debates currently exist over the interaction between emotion and memory. For example, there is debate about the accuracy of emotional memories: although strong emotional memories, including flashbulb memories, may subjectively feel like they are very accurate, there is evidence that not all details are preserved accurately. Additionally, there is debate over how advancing age affects memory for emotional information, both in the formation of new memories and retrieval of old emotional memories.

One particular debate, which we will examine in more detail here, is how emotion affects the encoding and preservation of the details of a memory: information that is not necessarily central to the emotional event or stimulus itself, but is peripherally associated. Although there is little disagreement that emotional information typically receives a benefit in memory both in and out of the lab, there is less agreement over whether emotion preserves all of the details of a memory equally. Research has specifically examined how presence of emotional information affects memory for other, $\rightarrow$ peripheral information.

Some researchers believe that the presence of emotional information induces a memory trade-off: as in the weapon focus effect, our memory is enhanced for emotionally arousing information (e.g., a gun), but that benefit comes at the decrement for other peripheral, but potentially also important, information (e.g., the face of the person pointing the gun at you). There is substantial evidence supporting the notion that emotional information impedes the binding of other information. One demonstration of this effect comes from Elizabeth Kensinger and colleagues (2009), who asked participants to view scenes that contain either emotional or neutral objects. For example, participants may view a forest, either with a chipmunk (neutral) or a snake (emotional) in the foreground. When memory is then probed separately for both the objects and backgrounds, a memory decrement is observed for backgrounds that were paired with emotional, rather than neutral, objects.

However, other researchers have shown evidence that the presence of an emotional stimulus actually facilitates the binding of contextual details. For instance, Donald MacKay and others (2004) showed that participants more readily bound contextual details (such as the task type and font color of words in a number of laboratory tasks) to "taboo" words words that were explicit or otherwise highly arousing than to neutral words.

One way to reconcile such seemingly disparate findings may be to consider spatial or temporal proximity of the "central," emotional stimulus and the peripheral details being examined. In a review that summarizes many of the above findings, Mara Mather (2007) provides a concise explanation. She suggests that 
when peripheral details are inextricably linked to the emotional stimulus, those details will receive an emotional benefit in memory. For example, in the studies presented by MacKay's group, the peripheral details which show a memory enhancement - word color, which task the words appeared in, and so forth - are really features of the emotional stimulus itself. Word color, for example, does not appear in a different space or at a different time than the word itself; therefore, it is encoded as part of the arousing, taboo stimulus. On the other hand, trees that appear in the background behind a lunging snake are not encoded as features of the snake itself, and therefore show a memory decrement. As such, the fate of details that are peripheral to an emotional stimulus may be decided by how "relevant" those details are to the emotional information.

Much of the evidence for the above theories comes from studies where emotionally arousing information is contrasted against non-arousing information. However, as noted earlier, arousal is only one dimension of emotion; valence - whether something is pleasant or unpleasant - is also a critical factor in determining how information will be remembered. Barbara Fredrickson (Frederickson and Branigan 2005) has shown that while negatively valenced information often impedes the encoding of peripheral information, information that connotes positive emotion can "broaden and build" attention, making it easier to encode peripheral details.

\section{Cross-References}

- Arousal and Paired-Associate Learning

- Cognitive Aging

- Emotional Learning

\section{References}

Brown, R., \& Kulik, J. (1977). Flashbulb memories. Cognition, 5, 73-99.

Frederickson, B., \& Branigan, C. (2005). Positive emotions broaden the scope of attention and thought-action repertoires. Cognition and Emotion, 19, 313-332.

Kensinger, E. A. (2009). Phases of influence: How emotion modulates the formation and retrieval of declarative memories. In M. Gazzaniga (Ed.), The cognitive neurosciences IV. Cambridge, MA: MIT Press.

MacKay, D. G., Shafto, M., Taylor, J. K., Marian, D. E., Abrams, L., \& Dyer, J. R. (2004). Relations between emotion, memory, and attention: Evidence from taboo Stroop, lexical decision, and immediate memory tasks. Memory \& Cognition, 32, 474-488.
Mather, M. (2007). Emotional arousal and memory binding: An object-based framework. Perspectives on Psychological Science, 2, 33-52.

Öhman, A., Flykt, A., \& Esteves, F. (2001). Emotion drives attention: detecting the snake in the grass. Journal of Experimental Psychology: General, 130, 466-478.

Talmi, D., \& Moscovitch, M. (2004). Can semantic relatedness explain the enhancement of memory for emotional words? Memory \& Cognition, 32, 742-751.

\section{Emotional Mental Models}

\section{BRigitTe StANGL}

Institute for Tourism and Leisure Studies, University of Economics and Business, WU Vienna, Vienna, Austria

\section{Synonyms}

Emotion scheme; Emotional conceptual model; Personal scheme

\section{Definition}

Emotional mental models are one part of people's mental models intrinsically tied to cognitive models. Mental models stipulate that people have certain expectations/thoughts of how things should look like/ work and connect certain emotions with this. Mental models are a framework in the brain for new learning situations which are based on experiences/meanings and which are influenced by a persons' personality and the environment. Thereby, emotions and feelings are considered as emotional mental models while thoughts and beliefs are accounted for by cognitive models. In learning situations new information is compared with existing content (beliefs and emotions) and structures; then, an adapted cognitive and emotional mental model is generated. Human beings' feelings, reactions, and behavior toward stimuli (person/situation/product/brand/service) are guided by emotional mental models.

\section{Theoretical Background}

Mental models (Norman 1988) have been examined in various fields such as management, marketing, information systems, consumer behavior, psychology, education, and neuroscience. However, most scientists focus on cognitive models only, neglecting different 
underlying meanings of mental models such as attitudes, emotions and feelings, symbols, actions, goals, values, images, memories, visions, or representations of sensory experience (touch, taste, and smell). Only very little research explicitly takes into account cognitive and emotional mental models jointly. However, there is empirical evidence that cognitive and emotional mental models are not separable because both structure and content depend on each other and influence behaviors (Christensen and Olson 2002). Structure is about how information is organized in memory while content refers to personal meanings comprising thoughts/beliefs and emotions/feelings. LeDoux (1996) points out that there is only one conscious system which can be filled with trivial facts or with profound emotions. The basics for construction of emotions (nervous system, functionality of mental and physical processes) is determined in our genes but how we actually act, think, and feel depends on aspects learned, experiences gained, and meanings attached. Unconsciously emotions of others are imitated and emotional mental models are built and stored in the long-term memory. Cognitive processes are the repository of signals triggering emotional reactions and enable human beings to act appropriately in specific situations rather than just react. Among various types of cognition that activate emotions are appraisal, evaluation, comparison, categorization, belief, anticipation, or memory. Emotions in turn are the basis for motivation and selective perception/attention (Izard 1993). Mental models cause certain expectations/ thoughts of how things should look like/work and connect certain emotions with this. Consequently, a mental model is a cognitive and an emotional framework in the brain, influenced by person's personality (genes) and the environment including social variables. This framework is assessed and adapted (if required) in learning situations. Learning situations comprise all kind of stimuli (e.g., people, situations, and products). The initial mental model is used as a reference point in order to judge novel stimuli. Accordingly, appraising stimuli is a key factor in the adaptation process. The underlying procedure equates to a double-loop learning process. If a certain level of discrepancy between the initial model and the new stimuli is reached, underlying assumptions/meanings or more precisely cognitive and emotional models are adapted. Thereby, both cognitive and emotional mental models are activated at the same time. In doing so, connections from the emotional to the cognitive system are even stronger than the other way around (LeDoux 1996). After all, mental models allow people to understand the world, to grasp learning situations, as well as to predict outcomes/reactions/consequences without knowing all the details behind. Thus, mental models are cognitive and emotional abstractions that provide just enough information to understand a learning situation and to be able to detect how to behave (Norman 1988). Thus, mental models affect people's attitudes and behavior by either approaching or avoiding for instance relationship, purchase, usage, or learning (Mehrabian and Russell 1974). However, because the interpretation of new situations is based on only small hints and reasoned by analogy with former experiences, misinterpretations and wrong reactions might be the consequence. Nevertheless, positive or negative experiences impact on future behavior such as on solutions for problems, satisfaction, word-of-mouth, or loyalty (e.g., Oliver 1993). This unconsciously constantly ongoing process is summarized in Fig. 1.

Emotional mental models are highly personal and idiosyncratic. They influence experience, interaction, and behavior. In order to understand human beings' behavior, for instance, the degree of importance people place on something, how they think, and emotional

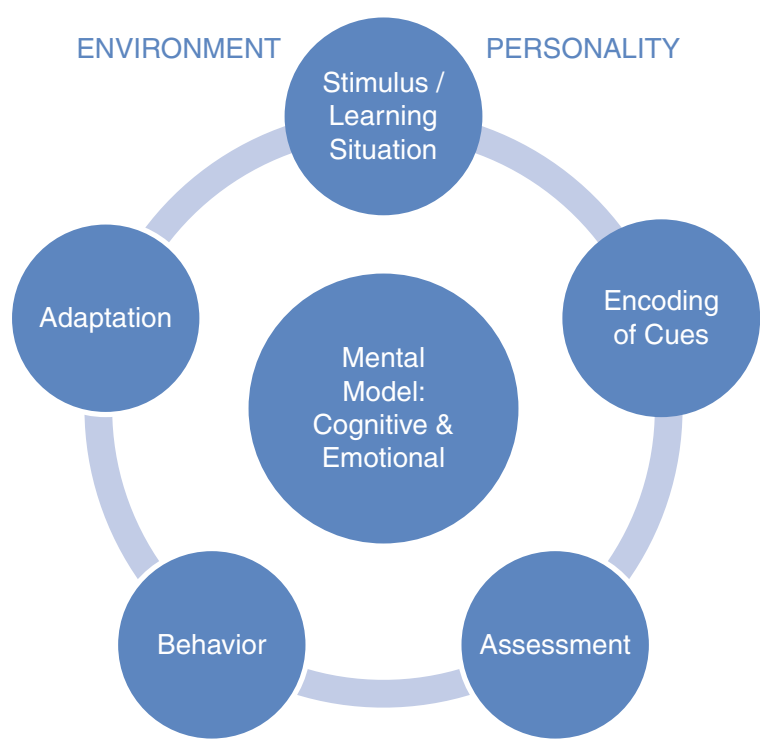

Emotional Mental Models. Fig. 1 Development/ transformation-process of cognitive and emotional mental models 
reactions as well as to decode their level of involvement, knowledge about both the underlying cognitive and emotional mental models is essential.

\section{Important Scientific Research and Open Questions}

Scientists have been investigating certain aspects of mental models for decades, albeit under diverse headings such as cognitive structure, expectation-disconfirmation, attitudes, beliefs, personality, or expertise. There are several attempts of linking the concepts of thoughts, feelings, and action (cognition, affect, and behavior), such as congruity theory, attributional theory of motivation, theory of planned behavior, or social cognitive theory. However, research on mental models as such mainly focuses on how information is structured in the memory like, for instance, hierarchical or as a network. Only very few concentrate on the ideas and concepts behind mental models. There are some attempts such as by Christensen and Olsen (2002) who revealed and mapped both sides of a mental model (i.e., cognitive and emotional) for highly motivated mountain bikers. Nonetheless, it is criticized that research does not focus on the meaning of a person/product/ brand/service but separates structure and content. However, conceptually both need to be considered together because if cognition and/or emotion changes, necessarily the structure changes too and vice versa. Reasons for this lack of connecting both might be complexity and measuring issues. Among the most popular methods used for revealing mental models are qualitative approaches such as laddering technique and means-end chain analysis. Another method using pictures as stimuli referred to as the Zaltman metaphor-elicitation technique (ZMET) is a worthwhile technique, however, used only rarely.

\section{Cross-References}

- Affective Dimensions of Learning

- Cognitive Models of Learning

- Double-Loop Learning

- Knowledge Representation

- Mental Models

- Mental Representation

\section{References}

Christensen, G. L., \& Olson, J. C. (2002). Mapping consumers' mental models with ZMET. Psychology \& Marketing, 19(6), 477-502.
Izard, C. E. (1993). Four systems for emotion activation: Cognitive and noncognitive processes. Psychological Review, 100(1), 68-90.

LeDoux, J. (1996). The emotional brain: The mysterious underpinnings of emotional life. New York: Simon\&Schuster.

Mehrabian, A., \& Russell, J. A. (1974). An approach to environmental psychology. Cambridge, Massachusetts: MIT Press.

Norman, D. A. (1988). The psychology of everyday things. New York: Basic Books.

Oliver, R. L. (1993). Cognitive, affective, and attribute bases of the satisfaction response. Journal of Consumer Research, 20(3), $418-430$.

\section{Emotional Quotient}

Social-Emotional Learning Scale

\section{Emotional Reactivity}

- Emotional Schema

\section{Emotional Schema}

\section{Norbert M. SeEL}

Department of Education, University of Freiburg, Freiburg, Germany

\section{Synonyms}

Affective schema; Emotional reactivity

\section{Definition}

An emotional schema is a particular totality of primarily affectively determined modes of responses and feelings toward people and events that can be transferred onto analogous situations and similar people. Emotional schemas are tightly integrated slot-filler structures of eliciting situations, subjective feelings, and expressive and autonomic activity. Basically we can distinguish between innate emotional schemas, cultural-specific emotional schemas, and individual emotional schemas. These groups of emotional schemas differ with regard to the commitment of their effects. 


\section{Theoretical Background}

Schema theory not only refers to cognition and information processing but rather also to emotions (Eckblad 1981). It was Piaget (1945/1952) who used the term "affective schemas" (synonymously with emotional schema) - not alternatively to "cognitive schemas" but rather as totalities of affectively determined responses and feelings to the environment. It may be sufficient to assert that an emotion is, first, experienced as a distinctive internal state, and second, associated with a unique schema.

Comparable with cognitive schemas the major function of emotional schemas consists in connecting sensory experiences and related affective responses by associating them with repeated events at similar times and locations. This results in quasi-emotional organizational units that functions as defaults of emotional schemas. They create invariance, reduce complexity, and facilitate the simplification and automatization of emotional responses. They are "templates" which can be applied immediately onto new experiences. Accordingly, the instantiation of an emotional response depends on the degree to which an event fits with an emotional schema.

An emotional schema "resembles recognition and intuitive preference, not reasoning, because there is no need for the eliciting stimulus or choice process to enter conscious awareness. Attention is required only to bring the eliciting stimulus into the receptor field. Once a component of the schema is activated, the other components are activated instantly" (Leventhal 1984, p. 276). As a consequence, schematic processes are, in general, rapid and automatic.

The learning-dependent change of schemas presupposes an incongruity between an existing schema and new information, that is, between standard and input. Piaget (1952) argued that various affective and attention states are localized in relation to the structural development of a schema. In the course of generalizing assimilation new sections of the environment are continuously being fed into the cognitive structure and repeated encounters will lead to differentiation, recognition, and pleasure. Further encounters with similar information lead to the establishment of mastery in the sense of effortless, practiced recognition or familiarity. We can observe this when small children are exposed to the letters of the alphabet. Initially, they are inattentive to them, but then gradually the interest and attention toward letters increase, accompanied by a growing differentiation. There is pleasure at each new letter which the child is able to recognize. Finally, as mastery is reached, in the sense that all letters are known and recognized, interest in the letters as such ceases.

If incongruities are introduced after mastery has been reached, spontaneous repetition is instigated until mastery again is established. In a later stage larger incongruities are tolerated and eventually enjoyed: novel and more complex information excite interest. As schemas become more elaborated their activation no longer claims attention; they form a part of the maintenance schemas or the general repertory of knowledge and skills of the person.

Whenever assimilation in some schema fails, this schema enters a state of disequilibrium which in turn evokes arousal. The term "motive" can be used to denote the presence of disequilibrium - whenever an attempt at assimilation fails and corrective attempts are not immediately successful, a motive will be originated. This argumentation follows Berlyne's (1971) views on the central role of arousal in curiosity motivation and active stimulus seeking. This role of arousal in schema theory may be formulated as follows:

1. Arousal is assumed to increase with the degree of incongruity in schemas.

2. High levels of incongruity are innately aversive. This effect may be mediated by arousal (as proposed by Berlyne) as well as by other factors, such as a limited processing capacity.

3. It is assumed that the stronger a schema is, the larger will be the effect of incongruity in that schema, and the more arousal will be generated.

4. Incongruity occupies processing capacity and produces arousal to some function, whereby the amount of arousal may vary from time to time and from person to person. For instance, introverts may have a higher level of arousal than ambiverts and ambiverts than extraverts.

5. Arousal and incongruity are foremost to be regarded as two facets of a unitary process, the activation of schemata.

In Berlyne's terms, the common characteristic of collative variables (novelty, complexity, etc.) is that 
they induce (a cognitive) conflict which in turn increases arousal. This mediates the affective reaction, for instance curiosity. The important property that complex, novel, incongruous, etc., objects have in common is that they resist immediate perceptual or conceptual assimilation. The assimilation resistance may have various causes, but in all cases the immediate result is some degree of disequilibrium in the schema where the assimilation occurs. The relationship between assimilation resistance, affective response, and curiosity motivation can be described as in Fig. 1.

\section{Important Scientific Research and Open Questions}

An emotional schema refers to an emotion-specific set of interrelated information including vocabulary, semantic and conceptual knowledge, memories of prior experiences, and physiological sensations. Since the mid-1970s, cognitive psychologists have been advocating cognitive theories of emotion. From this perspective two research approaches have arisen, one of which focuses on the influence of emotional schemas on information processing and learning. This approach corresponds with the idea that an individual is experiencing a particular mood while selectively attending to and learns material that is similar in the affective tone. The material is presumed to be included in the schema through prior association with the emotion, and its biased processing is referred to as moodcongruent memory. An alternative research approach focuses on the memorial consequences of emotion as a distinctive state and is a natural extension of research on state-dependent learning both in animals and in humans. The phenomenon of interest, $>$ mooddependent memory, is evident when memory for a past event is enhanced by the presence of a mood that is similar to one that was present during the original encoding of that event. Importantly, the enhanced memory should occur for all aspects of the event, regardless of the affective valence of the material itself. Thus, when subjects learn material while sad, the presence of a sad mood later on will enhance the memory for that material, regardless of whether the emotional valence of the material is happy, sad, or neutral.

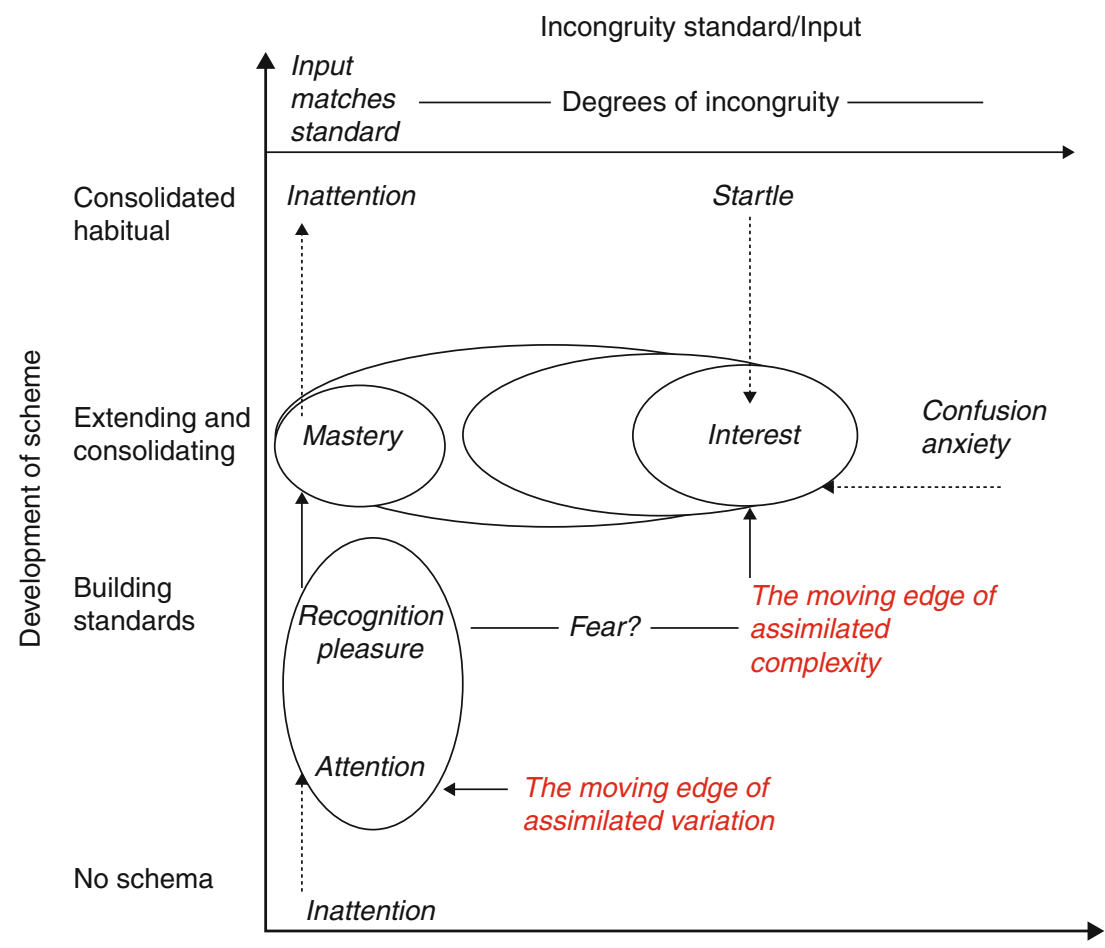

Emotional Schema. Fig. 1 Assimilation resistance and emotions (Eckblad 1981, p. 39) 
Emotions had been studied extensively in both animals (e.g., rats) and humans, but most studies concern simple and basic emotional responses, such as fear and stress. Emotions of animals and humans are quite complicated, subtle, and nuanced due to the experience of a wired range of emotional phenomena. However, the neurophysiologic basis of emotions and emotional schemas, respectively, are not precisely specified to date (Lewis and Haviland-Jones 2004). It is quite easy, for instance, to discern anxiety and infuriation, but the physiological arousal we perceive is very similar and we interpret the physiological response in accordance with the situation. This idea corresponds with the James-Lange theory of emotion (Cannon 1987) which finally leads to the sequence "Event $==>$ arousal $==>$ interpretation $==>$ emotion."

Although we know that emotions are evoked by and processed in anatomical circuits in the prefrontal cortex, ventral striatum, and the amygdala, emotions are higher-order cognitive interpretations. That is, an activated amygdala-frontal lobe circuit could be interpreted, and hence experienced, as fear or as anger or even as surprise or joy, depending upon which cognitive schema was active at the time. Phelps et al. (1998) examined the role of the amygdala in emotional memory. The authors postulate that the amygdala may contribute to the formation of episodic memories through the organization of "an organizing principle such as a schema or category" (p. 536). This finding tends to lend credence to the formation of schemas around a nodal point of affectively charged experience, with the participation of the amygdala.

Emotional schemas can be conceived as the result of the interactions between physiological reactions and cognitive schemas that respond to particular stimuli from the environment. At the moment, neuroscience can assess activities of particular areas in the brain in which autonomic reactions to environmental stimuli occur, but how cognitive and emotional schemas work is not sufficiently investigated as yet. However, several models of schema development count on the input of emotional experience in the formation of enduring associative complexes. These investigations designate a promising field of future research with the aim to close the gap between cognitive psychology and neuropsychology.
Another central issue of research on emotional schemas concerns working with emotions in psychotherapy (Greenberg and Paivio 1997) in order to treat a wide range of complaints, including depression, anxiety, and other maladaptive emotional schemas. In this field of application, emotions are not considered as irrational feelings but rather - in accordance with schema theory - as organizers of past experiences, memories, fears, and dreams that vary between and within individuals. Schemas are organizers not only of emotions, but also of identity and self-concept. The major assumption in working with emotions in psychotherapy is that maladaptive emotional schemas can be restructured. A successful treatment contains (1) bonding, (2) evoking and exploring emotional experiences of the past and present, and (3) restructuring the emotional schema (Greenberg and Paivio 1997). This emotionally focused therapy (EFT) operates on specific moment-to-moment interventions of the therapist who attunes to the slightest changes and shifts in the client's emotional expressions.

\section{Cross-References}

- Emotion Regulation

- Emotional Learning

- Emotional Mental Models

- Self-schema

\section{References}

Berlyne, D. E. (1971). Aesthetics and psychobiology. New York: Appleton.

Cannon, W. B. (1987). The James-Lange theory of emotions: a critical examination and an alternative theory. The American Journal of Psychology, 100, 567-586.

Eckblad, G. (1981). Scheme theory. A conceptual framework for cognitive-motivational processes. London: Academic.

Greenberg, L. S., \& Paivio, S. C. (1997). Working with emotions in psychiatry. New York: Guilford Press.

Leventhal, H. (1984). A perceptual motor theory of emotion. In K. R. Scherer \& P. Ekman (Eds.), Approaches to emotion (pp. 271-291). Hillsdale, NJ: Erlbaum.

Lewis, M., \& Haviland-Jones, J. M. (Eds.). (2004). Handbook of emotions (2nd ed.). Mahwah, NJ: Erlbaum.

Phelps, E. A., LaBar, K. S., Anderson, A. K., O’Connor, K. J., Fulbright, R. K., \& Spencer, D. D. (1998). Specifying the contributions of the human amygdala to emotional memory: A case study. Neurocase, 4, 527-540.

Piaget, J. (1945/1952). La formation du symbole chez l'enfant. Imitation, jeu et rêve, image et représentation. Neuchâtel: Delachaux \& Niestlé. 


\section{Emotion-Based Machine Learning}

\author{
Aladdin Ayesh
}

Department of Informatics, De Montfort University, Leicester, UK

\section{Synonyms}

Computational emotions; Emotion-induced adaptation; Mood congruency learning

\section{Definition}

An emotion may be defined as an elementary consciousness concept realized as a summarization state of continuous physical and mental processes as modeled in the brain or at a machine core. According to this definition emotion as an atomic element is a mental concept; however, as a state it could be either mental or physical. This is to account for the physiological manifestation of emotions. One may argue such manifestations are unconscious thus contradicts the definition given above. It is worth mentioning at this point, there is no single definition of emotions available that holds the wide community consensus. However, the definition provided here will serve us within this entry and comply with the common lines agreed by researchers in the various disciplines of interest.

Learning may be defined as the process of acquiring, extracting, organizing, optimizing, and fusing memory content for the inference of cognitive and behavioral responses. Cognitive responses can be in the form of object recognition, spatial associations, or reasoning ability. Behavioral responses often follow cognitive responses and are concentrated in action selection with varying degrees of rationality and reactivity. Again, this definition is for the purpose of this entry and attempts to unify the various facades of learning.

\section{Theoretical Background}

In addressing the theoretical background of emotionsbased machine learning, one has to address the terminology that is necessary for artificial emotional systems. A more extensive discussion is presented in (Ayesh 2009). Addressing terminology is often rare in reported computational emotions research. Nonetheless, terminological confusion is often observed in translating the psychological theories of emotions into computational models of emotions to be used in intelligent machines including machine learning.

Main terms used in emotion modeling can be summarized as follows: Emotion, Emotional State, Mood, Desire, Motivation, and Affect. The latest, Affect, lends itself to Affect Computing, which is concerned with motivators that may or may not include emotions. The two main terminologies from this list are Emotion and Emotional State. However, not all studies of computational emotions make this explicit distinction between these two notions.

Emotions may be viewed as atomic elements representing the interpretation of feelings or sensations. This school of computational emotions is a direct derivative from the psychological school of basic emotions initiated by Darwin and championed by the likes of Ekman (Strongman 2000).

If an agent has a sensation of pain that may be associated with basic emotions of anger and sadness, for example. The physical state of the agent, however, is pain. The basic emotions in themselves may not lead to any actions. Being sad or angry may not lead by itself to taking action, but the aggregation of these feelings may activate a state of mind or a trigger. Emotional state can be viewed to be that trigger to be used in control mechanism, thus it requires calculation from a number of factors. If one accepts the theory of a basic emotion, emotional state can be specified as an aggregation function of all basic emotions to create a frame of mind. This emotional frame of mind will have an impact on perceptual interpretation of situations and decisions and subsequently impact on learning style and activities, e.g., shallow exploration of knowledge vs. deep inductive/deductive inference based learning. The attraction of basic emotions theory is that it is easy to implement in intelligent machines. It is often implemented as an array of descriptors to tag events, objects and actions, or as threshold variables in the configuration space of the emotional agent providing control parameters. The second option is often where the distinction between emotion and emotional state becomes blurred, that is if emotional state is ever mentioned.

Studies in psychology of emotions have long disputed the theory of basic emotions, and alternatives 
were proposed (Strongman 2000). Two alternatives are worth mentioning: emotional spaces and appraisal based emotional theories. These alternatives started to seep through into computational emotions research leading to open questions and recent developments (Ayesh 2009). Some of these developments and discussions can be followed through the HUMAINE Network (http://emotion-research.net/).

Before concluding this section we need to relate emotions to learning. Emotions impact the two important aspects of learning: cognitive and behavioral. Emotions impact cognitive learning by associating memory elements with emotional descriptor. This emotional descriptor acts as perceptual definition that may lead the emotional agent to retrieve particular experiences, seek further information, hence, inference, or motivate learning activity such as imitation. Eich et al. (2000, pp. 92-114), provide a good example on mood congruency and learning. As the emotional agent seeks further information and alters its behavior in response to emotional memory, the behavioral learning starts to take place.

Emotion-based behavioral learning is most evident in conditioning or reinforcement learning, which is often referred to as Temporal Difference Learning (TDL) in machine learning literature. Reinforcement Learning (RL) in machines happens as a direct association between behavioral policy, to be learned, and its consequential events represented as states. By adopting rewards from psychological conditioning research (Forgas 2000; Eich et al. 2000), the RL algorithm presents bias toward one state over others giving higher priority to the associated behavior, hence learning the behavioral policy. Emotions play a big role in calculating the bias to be given to a particular action based on the reward received. Emotion-based RL algorithms imitate the natural process to direct machine adaptive process. However, this is not the only place where emotions play a role. As research on imitation-based learning progresses in social robots, emotions play an important role and underline the motivation module of such robots.

\section{Important Scientific Research and Open Questions}

There are open questions on emotions-based learning in every relevant field such as psychology, sociology, philosophy, neurology, and artificial intelligence.
We delineate our focus here to the open questions within intelligent machines research in relation to computational emotions and machine learning. We circumscribe the area further by addressing cognitive and behavioral learning as discreet categories. Of course, such demarcation is for presentational purposes only since any real separation, between the different disciplines and faces of learning and emotions, is impossible. It is worth noting that emotions exploration in machine learning is a very recent research area with very few disparate examples in strong contrast to its psychological counterpart, where emotions have been studied extensively within theories of learning and developmental psychology (Strongman 2000; Forgas 2000; Eich et al. 2000).

The first open question of relevance to cognitive learning, which is expected to remain so for some time, is emotion modeling. Most of the efforts in computational emotions are grouped around basic emotions theory and emotional descriptors such as the case that two subsequent questions arise. First, how can we encompass new theories of emotion psychology and depart from threshold computational representation of emotions that put the validity and fidelity of computational emotions in question? Second, how can we represent emotional descriptors in a generic fashion that is accessible for all cognitive systems?

In response to the first question of modeling, the basic emotions theory has been revisited on several occasions. Attempts are being made to explore other schools of emotion psychology (Ayesh et al. 2007; Ayesh 2009), particularly those based on emotional spaces. In emotional spaces, emotions are defined in relation to two- or three-dimensional spaces. Each axe within the emotional space represents a factor of emotion. For example, the simplest space to be established is positive-negative space. Other spaces may include control-valence, appraisal-anxiety, Watson's XYZ space of fear-rage-love, and in similar spirit Plutchik's threedimensional model (Strongman 2000, pp. 32, 49).

In response to the second question of defining emotional descriptors, several attempts are appearing in the context of emotional ontology and emotional labeling culminated in EmotionsML (http://www.w3. org/TR/emotionml/), which is being developed by a W3C working group. However, this question is far from being answered. An emotional ontology will 
be useful to cognitive learning of concepts and associations. Behavioral learning and adaptive systems will reap far less benefits, if any, from emotional ontology research.

In behavioral learning, the open questions are related to how encoded emotions may impact on action selection, consequential actions, and the creation and recognition of behavioral patterns. This links strongly to the emotional spaces research in which emotional signals can be fed in continuous models of behavior (Ayesh 2009) producing situated cognition (Ayesh et al. 2007). Also, physiological views of emotions are becoming important in considering learning behaviors and adapting abilities; thus genetic algorithms and neural network approaches to emotionbased learning-behavior are gaining momentum of their own (Kim and Lee 2008).

Finally, there are still open questions in psychology that are seeping through into computational emotions and emotion-based machine learning. The questions of most interest are those related to the inter-relation between emotions, cognition, and behavior especially circumscribed by factors of personality, perception, and the social dimension of emotions and learning. One particular style of learning, namely imitation, is gaining the interest of researchers especially with its potential educational applications. Emotion modeling and inference play an important role here in directing the learning activity and influence the user-machine interaction. This is fueled by the advances in gaming research (Kim and Lee 2008, and Ayesh et al. 2007). Within the context of serious gaming, computational emotions and emotion-based machine learning may have something to offer in return to psychologists and social scientists on the relation between emotion and learning.

\section{Cross-References}

- Adaptive Learning

- Emotional Schema(s)

- Mental Model

\section{References}

Ayesh, A. (2009). Swarms-based emotions modelling. International Journal of Bio-Inspired Computation, 1, 118-124.

Ayesh, A., Stokes, J., \& Edwards, R. (2007). Fuzzy individual model (FIM) for realistic crowd simulation: Preliminary results. Fuzzy Systems Conference (FUZZ-IEEE'07), London.
Eich, E., Kihlstorm, J. F., Bower, G. H., Forgas, J. P., \& Niedenthal, P. M. (2000). Cognition and emotion. New York: Oxford University Press.

Forgas, J. P. (Ed.). (2000). Feeling and thinking. New York: Cambridge University Press.

Kim, J.-H., \& Lee, C.-H. (2008). Multi-objective evolutionary generation process for specific personalities of artificial creature. IEEE Computational Intelligence Magazine, 3(1), 43-53.

Strongman, K. T. (2000). The psychology of emotion: Theories of emotion in perspective. Chichester: Wiley.

\section{Emotion-Induced Adaptation}

- Emotion-Based Machine Learning

\section{Emotions in Cognitive Conflicts}

\author{
AnNeKATHRin SChacht ${ }^{1}$, Werner SOMmer ${ }^{2}$ \\ ${ }^{1}$ CRC “Text Structures”, University of Göttingen, \\ Göttingen, Germany \\ ${ }^{2}$ Department of Psychology, Humboldt-Universität zu \\ Berlin, Germany
}

\section{Synonyms}

Affective responses; Incompatibility effects

\section{Definition}

Conflicts in the cognitive system of an individual arise when at least two incompatible response tendencies or motivational states are simultaneously active. Emotions are affective, intentional states, including feelings such as happiness, anger, sadness, or surprise, in response to the evaluation of a relevant external or internal event. It has been suggested that cognitive conflicts elicit (negative) emotions. Conversely, emotional states or moods may influence the processing of cognitive conflicts.

\section{Theoretical Background}

In recent years, the intimate relationship between emotions and cognition has become increasingly clear for many areas, for example, memory and creativity. This holds true also for cognitive conflicts, caused by incompatible response tendencies or motivational states. Such situations arise, for example, when the directions 
given to a driver by GPS and by a road sign differ, or when - in the classic Stroop task - the word "red" is printed in blue while the color of the ink is to be named. In order to perform efficiently, these conflicts have to be detected and resolved. Following a conflict often adaptations of the processing routines occur, presumably, in order to optimize the future handling of conflict situations. The role of emotions in the processing of cognitive conflicts can be viewed from two angles: (a) whether and how conflicts induce emotions, and (b) whether and how emotions affect conflict processing.

As to the emotional consequences of conflicts, Botvinick (2007) pointed out that conflicts impose increased demands on cognitive processing relative to conflict-free tasks or situations. Based on the idea that the cognitive system tends to avoid demanding situations (law of least effort), Botvinick suggested that cognitive conflicts would register as costly and aversive events. Consequently, the occurrence of conflicts would trigger a form of avoidance learning, manifesting as a bias toward tasks and strategies that minimize the risk for conflict and hence increased mental effort. In this context, cognitive conflicts have been placed among a larger set of value-laden outcomes, for example, monetary loss, negative feedback, social exclusion, or pain, all of which are registered by the anterior cingulate cortex (ACC). The ACC, located on the medial surface of the frontal lobes, lies at the crossroads of three vast anatomico-functional systems: the motor, limbic, and prefrontal cortices and has been shown to play a crucial role in conflict monitoring (see below). Thus, the ACC has been suggested as a gateway interconnecting emotional and cognitive components of the mind (Devinsky et al. 1995).

An alternative approach concerning the elicitation and differentiation of emotions in the context of conflicts is provided by appraisal theories. Here, a sequential series of appraisal checks about the novelty, intrinsic pleasantness, goal significance, agency, coping potential, and compatibility with personal and social standards defines whether and which specific emotions emerge. Emotions appear as episodes of interrelated components or sub-events, including the (cognitive) evaluation of the relevant event, the subjective experience, its expression, and specific physiological changes, as well as action tendencies. Within his Component Process Model, Scherer (2001) proposed that the evaluation of goal/ need conduciveness versus obstructiveness is based on the prior goals and needs of the organism and provides the organism with information about specific adaptive responses and adjustments. Since cognitive conflicts represent events of high cognitive demands, threatening goal attainment, they should be evaluated as goal/ need-obstructive for the organism and thus obtain a negative emotional value.

Although effects of emotions have been shown for many cognitive processes (Ashby et al. 1999) and also for conflict processing (see below), theoretical explanations of these effects are less well developed. Thus, it has been suggested that positive emotions increase the availability of the neurotransmitter dopamine in frontal brain areas, especially in the ACC, enhancing cognitive flexibility and allowing to reorganize behavior (Dreisbach and Goschke 2004).

\section{Important Scientific Research and Open Questions}

The experimental investigation of cognitive conflicts has focused on several classical paradigms, where overt responses to incompatible trials are contrasted with responses to compatible trials. Most notable examples are the Simon, Stroop, or Eriksen flanker tasks. Common to incompatible trials in these tasks is the overlap of usually two incongruent response tendencies due to the simultaneous activation of task-relevant and other, task-irrelevant but sometimes also - relevant, stimulus features. These incongruencies impair performance as evident in increased reaction times and error rates.

The assumption that cognitive conflicts represent unpleasant or aversive events has been based on empirical ties between conflicts and errors on the one hand and errors and emotions on the other hand. Current research indicates close functional and neurophysiological links between the processing of conflicts and response errors as revealed in experimental paradigms. This idea is supported by strong neuroanatomical relations between the brain areas involved in monitoring conflicts and errors in the ACC. Two prominent components of event-related brain potentials have been shown to reflect errors or conflict monitoring: the socalled error-related negativity (ERN or $\mathrm{Ne}$ ), elicited by incorrect responses, and the N2, which is enhanced in correctly processed incongruent or incompatible conflict trials. Both components show similar distributions at the scalp surface and are presumably generated by the same or at least closely located neural generators 
within the ACC. On the other hand, erroneous responses have been reported to be accompanied by emotions in terms of enhanced activity of the autonomic nervous system (ANS), and ERN amplitudes can be modulated by interindividual differences in emotion-related states and traits. Based on these functional and neuroanatomical links, it has been assumed that cognitive conflicts might be accompanied by emotional responses as well.

Direct evidence about the relationship between conflicts and emotion has been obtained by peripheral physiological recordings during experimental tasks. For the so-called Go/Nogo task, where, in nogo trials, the preponderant response tendency is considered to be in conflict with the need to inhibit this tendency, there is consistent evidence against the aversive valence of this kind of conflict. Instead, nogo trials seem to be experienced as a relatively pleasant interruption of the monotonous response routine. In contrast, for Simontype conflicts, no evidence for any emotional involvement was obtained in an otherwise very similar design (Schacht et al. 2010). These findings strongly argue against an aversive quality of emotions in cognitive conflicts in any general sense.

There is some empirical evidence in favor of the modulation of conflict processing by emotional states or mood. Moods are relatively diffuse affective states of longer duration and lower intensity than emotions and may be present without a concrete eliciting event. Positive moods, especially, when activating rather than calm and relaxed, are considered to be advantageous for creativity and have been shown to enhance cognitive fluency/flexibility in the context of executive functions. Positive but not negative mood was found to eliminate the Stroop effect, provided that intentional memory was sufficiently loaded by preparing for several tasks in a row, and positive but not negative pictures reduced the costs of incompatible responses (Dreisbach and Goschke 2004). Positive mood, induced by arbitrary rewards, was also suggested to counteract the need for conflict adaptation induced by the presumed aversiveness of conflict.

In principle, the idea that - like interpersonal conflicts - also cognitive conflicts might induce emotions and, in turn, might be modulated by emotions appears to be plausible. However, the empirical evidence for this idea is still scarce and heterogeneous. Future work should therefore investigate the boundary conditions and constraints for these relationships. Thus, it is conceivable that not all cognitive conflicts show a relationship to emotion. The interplay of cognitive conflicts and emotions might depend, for example, on the type of conflict, on the relevance of the conflictrelated action for the goal of the individual, and on the kind of emotion involved.

\section{Cross-References}

- Cognitive Conflict and Learning

- Creativity, Problem Solving and Feeling

- Emotions: Functions and Effects on Learning

- Mood and Learning

- Neural Substrates of Avoidance Learning

- Neuropsychology of Emotion

- Neuropsychology of Learning

\section{References}

Ashby, F. G., Isen, A. M., \& Turken, U. (1999). A neuropsychological theory of positive affect and its influence on cognition. Psychological Review, 106, 529-550.

Botvinick, M. M. (2007). Conflict monitoring and decision making: Reconciling two perspectives on anterior cingulate function. Cognitive, Affective \& Behavioral Neuroscience, 7, 356-366.

Devinsky, O., Morrell, M. J., \& Vogt, B. A. (1995). Contributions of anterior cingulate cortex to behaviour. Brain, 118, 279-306.

Dreisbach, G., \& Goschke, T. (2004). How positive affect modulates cognitive control: Reduced perseveration at the cost of increased distractibility. Journal of Experimental Psychology. Learning, Memory, and Cognition, 30, 343-353.

Schacht, A., Dimigen, O., \& Sommer, W. (2010). Emotions in cognitive conflicts are not aversive but are task specific. Cognitive, Affective \& Behavioral Neuroscience, 10, 349-356.

Scherer, K. R. (2001). Appraisal considered as a process of multi-level sequential checking. In K. R. Scherer, A. Schorr, \& T. Johnstone (Eds.), Appraisal processes in emotion: Theory, methods, research (pp. 92-110). Oxford: Oxford University Press.

\section{Emotions: Functions and Effects on Learning}

\author{
Reinhard Pekrun
}

Department of Psychology, University of Munich, Munich, Germany

\section{Definition}

Emotions serve to initiate and coordinate individual reactions to important events and objects. They consist 
of a number of interrelated component processes, including affective, cognitive, physiological, motivational, and expressive components (Kleinginna and Kleinginna 1981). For example, a student's anxiety before an exam can be comprised of nervous, uneasy feelings (affective); worries about failing the exam (cognitive); increased physiological activation (physiological); impulses to escape the situation (motivation); and anxious facial expression (expressive).

As compared to intense emotions, moods are of lower intensity and lack a specific referent. Some authors define emotion and mood as categorically distinct (see Rosenberg 1998). Alternatively, since moods show a similar profile of components and similar qualitative differences as emotions (as in cheerful, angry, or anxious mood), they can be regarded as low-intensity emotions (Pekrun 2006). Different positive and negative emotions and moods are compiled in the more general constructs of positive and negative affect (Tellegen et al. 1999). All of these emotion constructs can relate to momentary emotional episodes (state emotions) or to dispositional tendencies to experience momentary emotions (trait emotions; e.g., trait anxiety; Spielberger et al. 1976).

Three dimensions describing emotions are valence, activation, and object focus. In terms of valence, positive emotions can be distinguished from negative emotions, such as pleasant enjoyment versus unpleasant frustration during learning. In terms of activation, activating emotions are distinguished from deactivating emotions, such as activating excitement versus deactivating relaxation. With regard to object focus, achievement emotions and epistemic emotions are especially important for learning. Achievement emotions pertain to achievement activities and their outcomes, such as enjoyment or boredom experienced during learning activities, and hope, pride, anxiety, or shame related to the success and failure outcomes of these activities. Epistemic emotions (Pekrun 2010) are related to the cognitive aspects of processing material to be learned, such as surprise triggered by unexpected information, curiosity about a problem, interest in a topic, frustration due to obstacles, or delight when a problem has been solved.

\section{Theoretical Background}

Research on emotions and learning was initiated by studies on test anxiety. This emotion was first investigated in the 1930s (Brown 1938) and obtained widespread attention after Mandler and Sarason (1952) had published their seminal article on anxiety and learning. Since the 1980s, a second important research tradition has emerged that attends to the effects of positive versus negative moods on cognitive processes, such as memory storage and retrieval, cognitive problem solving, and social judgment. Whereas test anxiety research focused on the effects of one specific, discrete emotion, mood research analyzed the effects of broad positive and negative mood states.

Most theories of test anxiety posit that anxiety interferes with performance on complex and difficult cognitive tasks (interference and attentional deficit models; Eysenck 1997; Wine 1971). Anxiety is thought to require cognitive resources, thereby reducing working memory capacity needed for the completion of such tasks. In contrast, performance on simple, easy, and repetitive tasks may not suffer or is even enhanced. The cognitive components of anxiety, such as worries about possible failure and task-irrelevant thinking, are thought to be responsible for the performanceimpairing effects of this emotion.

For positive and negative affect more broadly, experimental mood researchers have proposed various theories explaining effects on cognitive processes. First, in line with test anxiety theories, moods and emotions have been posited to consume cognitive resources by directing attention toward the object of emotion. Consumption of cognitive resources for task-irrelevant purposes implies that fewer resources are available for task completion, thereby negatively impacting performance (Ellis and Ashbrook 1988; Meinhardt and Pekrun 2003). Second, mood is expected to induce mood-congruent memory recall (e.g., Levine and Burgess 1997), whereby positive mood facilitates retrieval of positive self-related and task-related information, and negative mood facilitates the retrieval of negative information.

Mood has been proposed to influence cognitive problem solving, with positive mood promoting relational, flexible, and creative ways of solving problems, and negative mood promoting item-specific, detailoriented, and analytical ways of thinking. For example, in mood-as-information approaches (Clore and Huntsinger 2007), it is presumed that positive affective states signal that "all is well," whereas negative states imply that something is going wrong. "All is well" 
conditions imply safety and the discretion to creatively explore the environment, broaden a person's cognitive horizon, and build new actions, as addressed by Fredrickson's (2001) "broaden-and-build" metaphor of the effects of positive emotions. In contrast, if there are problems threatening well-being and agency, as signaled by negative affective states, it may help to focus on these problems in analytical, cognitively cautious ways.

All of these mechanisms can play a role in the effects of emotions on learning. In Pekrun's (2006) controlvalue theory of achievement emotions, it is proposed that three groups of mediating processes are especially important, including (a) the availability of cognitive resources enabling individuals to focus attention on learning tasks; (b) interest and motivation to perform these tasks; and (c) use of cognitive and metacognitive strategies for learning, including the self-regulation of learning. To explain the effects of emotions on these processes and resulting learning outcomes, the controlvalue theory argues that the valence and activation dimensions of emotions are most important, suggesting that four emotion categories should be distinguished (positive activating, positive deactivating, negative activating, and negative deactivating as depicted in Table 1).

Positive activating emotions such as enjoyment of learning are posited to (a) preserve cognitive resources and focus attention on the task, (b) promote the development of interest and intrinsic motivation, and (c) facilitate the use of flexible cognitive strategies (such as elaboration and organization of task material) and selfregulation. As such, these emotions should exert positive effects on learning under most task conditions.

Emotions: Functions and Effects on Learning. Table 1 Valence $x$ activation taxonomy of emotions

\begin{tabular}{|l|l|l|}
\hline & Positive $^{\mathrm{a}}$ & Negative $^{\mathrm{b}}$ \\
\hline Activating & Enjoyment & Anger \\
\cline { 2 - 3 } & Hope & Anxiety \\
\cline { 2 - 3 } & Pride & Shame \\
\hline Deactivating & Relief & Hopelessness \\
\cline { 2 - 3 } & Relaxation & Boredom \\
\hline
\end{tabular}

${ }^{\text {a }}$ Positive = pleasant emotion

${ }^{\mathrm{b}}$ Negative $=$ unpleasant emotion
In contrast, negative deactivating emotions such as boredom and hopelessness are posited to uniformly impair performance by reducing cognitive resources, undermining both intrinsic and extrinsic motivation, and promoting superficial information processing.

For positive deactivating emotions such as relief and relaxation, and for negative activating emotions such as anxiety, shame, and anger, effects are expected to be more complex. For example, anxiety and shame are thought to produce task-irrelevant thinking and worries about failure, thus reducing cognitive resources available for task purposes, and to undermine interest and intrinsic motivation. On the other hand, anxiety and shame are expected to induce motivation to avoid failure and to facilitate the use of rigid strategies such as rehearsal of learning material. These propositions imply that the effects of these emotions on performance are likely to depend on task conditions and may well be varied.

\section{Important Scientific Research and Open Questions}

Over the past 60 years, more than 1,000 empirical studies have dealt with test anxiety and its relationships with learning (Zeidner 1998). In contrast, for emotions other than anxiety and for general positive versus negative affect, the available evidence is limited. During the past 20 years, however, empirical research has begun to recognize the critical importance of various emotions and to investigate their relationships with learning (Efklides and Volet 2005; Linnenbrink 2006; Linnenbrink-Garcia and Pekrun 2011; Pekrun and Frese 1992; Pekrun 2010; Schutz and Pekrun 2007).

Empirical evidence on the effects of positive emotions corroborates that activating positive emotions can enhance learning and performance (Pekrun 2010). Specifically, K-12 and college students' enjoyment of learning, hope, and pride were found to correlate positively with interest, effort invested in studying, elaboration of learning material, and self-regulation of learning. Furthermore, these emotions correlated positively with students' learning outcomes. Consistent with evidence on discrete emotions, general positive affect has also been positively linked to students' cognitive engagement in some studies (Linnenbrink 2007). However, a few studies have found null relations between activating positive emotions and learning (Linnenbrink 2007; Pekrun et al. 2009). Moreover, caution should 
be exercised when interpreting the reported correlations, because relationships between emotions and performance could be due not only to effects of emotions on performance, but also to effects of performance attainment on emotions, implying reciprocal rather than unidirectional causation.

Research examining the effects of negative emotions on learning has focused primarily on anxiety, targeting test anxiety as well as subject-specific anxiety, such as mathematics anxiety. In line with interference theories of anxiety, empirical research has shown that anxiety impairs performance on complex or difficult tasks that demand cognitive resources (e.g., difficult intelligence test items). Consistent with these findings, field studies have shown that test anxiety correlates moderately negatively with students' learning outcomes. In general, 5$10 \%$ of the variance in students' achievement scores is explained by self-reported anxiety (Hembree 1988; Zeidner 1998).

Again, to interpret the correlational evidence, reciprocal causation has to be considered. Relationships between test anxiety and achievement may be caused by effects of performance (i.e., success vs. failure) on the development of test anxiety, in addition to effects of anxiety on performance. The scarce longitudinal evidence available suggests that test anxiety and students' learning outcomes are in fact linked by reciprocal causation across school years (Meece et al. 1990; Pekrun 1992). Furthermore, correlations with performance variables have not been uniformly negative across studies. Zero and positive correlations have sometimes been found, in line with the view that anxiety can exert varied effects on learning. Anxiety is likely to have deleterious effects for many students, but it may induce motivation to study harder, and thus facilitate performance, for individuals who are more resilient.

Few studies have addressed the effects of negative activating emotions other than anxiety, such as shame and anger. Shame is at the core of negative feelings of self-worth (Covington and Beery 1976) and was regarded as central to the fear of failure motive in traditional achievement motivation theory (Heckhausen 1991). Similar to anxiety, students' shame and anger related to failure have been found to correlate positively with task-irrelevant thinking, and negatively with academic interest, motivation, and selfregulation of learning. Correlations with learning outcomes also tend to be negative (Pekrun 2010).
However, as with anxiety, the underlying pattern of functional mechanisms may be complex and imply more than just negative effects. For example, Turner and Schallert (2001) showed that students who experienced shame following negative exam feedback increased their motivation when they continued to be committed to future academic goals and believed these goals were attainable. It is likely that shame and anger are detrimental for motivation and performance under many conditions, but can translate into increased task motivation when expectancies for agency and success are favorable.

For negative deactivating emotions such as boredom and hopelessness, the evidence is scant as well. In experimental research, boredom was analyzed as being induced by simple, repetitive tasks, such as assemblyline, vigilant, or data entry tasks. Boredom was found to reduce performance on these tasks (Fisher 1993). In a few empirical studies targeting performance correlates in students, boredom was negatively related to attention, intrinsic motivation, use of flexible learning strategies such as elaboration and organization of learning material, self-regulation of learning, and students' learning outcomes (e.g., Pekrun et al. 2010; Watt and Vodanovich 1999). Similarly, students' hopelessness related uniformly negatively to measures of students' motivation, study behavior, and academic performance, pointing to the potentially devastating influence of this emotion.

In sum, in line with the propositions of Pekrun's (2006) control-value theory, the available evidence suggests that activating positive emotions, such as enjoyment of learning, can have beneficial effects on learning. However, the evidence on positive emotions to date is too scant and not yet sufficiently consistent to warrant firm conclusions. Deactivating negative emotions, such as hopelessness and boredom, show the opposite pattern of associations, in that they relate uniformly negatively to measures of learning and performance. In contrast, activating negative emotions, such as anxiety, shame, and anger, likely exert more complex effects. While these emotions promote taskirrelevant thinking and reduce interest and flexible thinking, they can also strengthen effort to avoid failure and facilitate the use of more rigid rehearsal strategies. From an educator's perspective, however, any immediate benefits of emotions like anxiety or shame are outweighed by their overall negative effects on learning 
for the vast majority of students. Furthermore, negative emotions can have severe consequences for students' well-being and health (Zeidner 1998), thus indicating an urgent need to ameliorate excessive negative emotions in students' academic careers.

Given the limited number of available studies on the relevance of emotions (other than anxiety) for learning, more research is clearly needed. This research should attend to the functional differences between various discrete emotions and should analyze the role of these emotions for the diverse cognitive and motivational processes underlying learning, such as attention, use of strategies, memory storage and retrieval, as well as intrinsic and extrinsic motivation to learn. To this end, future research should use multiple methodologies and theoretical perspectives, and should pay attention to emerging trends in emotion research and learning research (Pekrun and Schutz 2007). Specifically, neuroscientific perspectives should be used to disentangle the relationships between emotions and learning (Immordino-Yang and Sylvan 2010), and these relationships should be analyzed for both traditional classrooms and nontraditional settings such as computer-based learning environments (D’Mello et al. 2009).

\section{Cross-References}

- Affective and Cognitive Learning in the Online Classroom

- Affective and Motivational Dispositions of/for Learning

- Affective Dimensions of Learning

- Affective Priming and Learning

- Emotion Regulation

- Emotional Intelligence and Learning

- Emotional Schema(s)

- Emotion-Based Learning

\section{References}

Brown, C. H. (1938). Emotional reactions before examinations: II. Results of a questionnaire. The Journal of Psychology, 5, 11-26.

Clore, G. L., \& Huntsinger, J. R. (2007). How emotions inform judgment and regulate thought. Trends in Cognitive Sciences, 11, 393-399.

Covington, M. V., \& Beery, R. G. (1976). Self-worth and school learning. New York: Holt, Rinehart \& Winston.

D’Mello, S. K., Craig, S. D., \& Graesser, A. C. (2009). Multi-method assessment of affective experience and expression during deep learning. International Journal of Learning Technology, 4, 165-187.
Efklides, A., \& Volet, S. (Eds.). (2005). Feelings and emotions in the learning process [special issue]. Learning and Instruction, 15, 377-515.

Ellis, H. C., \& Ashbrook, P. W. (1988). Resource allocation model of the effect of depressed mood states on memory. In K. Fiedler \& J. Forgas (Eds.), Affect, cognition, and social behavior. Toronto: Hogrefe International.

Eysenck, M. W. (1997). Anxiety and cognition. Hove, East Sussex, UK: Psychology Press.

Fisher, C. D. (1993). Boredom at work: a neglected concept. Human Relations, 46, 395-417.

Fredrickson, B. L. (2001). The role of positive emotions in positive psychology: the broaden-and-build theory of positive emotions. The American Psychologist, 56, 218-226.

Heckhausen, H. (1991). Motivation and action. New York: Springer.

Hembree, R. (1988). Correlates, causes, effects, and treatment of test anxiety. Review of Educational Research, 58, 47-77.

Immordino-Yang, M. H., \& Sylvan, S. (2010). Admiration for virtue: neuroscientific perspectives on a motivating emotion. Contemporary Educational Psychology, 35, 110-115.

Kleinginna, P. R., \& Kleinginna, A. M. (1981). A categorized list of emotion definitions, with suggestions for a consensual definition. Motivation and Emotion, 5, 345-379.

Levine, L. J., \& Burgess, S. L. (1997). Beyond general arousal: effect of specific emotions on memory. Social Cognition, 15, 157-181.

Linnenbrink, E. A. (Ed.). (2006). Emotion research in education: Theoretical and methodological perspectives on the integration of affect, motivation, and cognition [Special issue]. Educational Psychology Review, 18 (4), 307-314.

Linnenbrink, E. A. (2007). The role of affect in student learning: A multi-dimensional approach to considering the interaction of affect, motivation, and engagement. In P. A. Schutz \& R. Pekrun (Eds.), Emotion in education (pp. 107-124). San Diego, CA: Academic.

Linnenbrink-Garcia, L., \& Pekrun, R. (2011). Students' emotions and academic engagement [Special issue]. Contemporary Educational Psychology, 36(1).

Mandler, G., \& Sarason, S. B. (1952). A study of anxiety and learning. Journal of Abnormal and Social Psychology, 47, 166-173.

Meece, J. L., Wigfield, A., \& Eccles, J. S. (1990). Predictors of math anxiety and its influence on young adolescents course enrollment intentions and performance in mathematics. Journal of Educational Psychology, 82, 60-70.

Meinhardt, J., \& Pekrun, R. (2003). Attentional resource allocation to emotional events: An ERP study. Cognition and Emotion, 17, 477-500.

Pekrun, R. (1992). The expectancy-value theory of anxiety: Overview and implications. In D. G. Forgays, T. Sosnowski, \& K. Wrzesniewski (Eds.), Anxiety: Recent developments in selfappraisal, psychophysiological and health research (pp. 23-41). Washington, DC: Hemisphere.

Pekrun, R. (2006). The control-value theory of achievement emotions: Assumptions, corollaries, and implications for educational research and practice. Educational Psychology Review, 18, 315-341. 
Pekrun, R. (2010). Academic emotions. Invited chapter, prepared for T. Urdan (Ed.), APA educational psychology handbook (Vol. 2). Washington, DC: American Psychological Association.

Pekrun, R., \& Frese, M. (1992). Emotions in work and achievement. In C. L. Cooper \& I. T. Robertson (Eds.), International Review of Industrial and Organizational Psychology (Vol. 7, pp. 153-200). Chichester, UK: Wiley.

Pekrun, R., \& Schutz, P. A. (2007). Where do we go from here? Implications and future directions for inquiry on emotions in education. In P. A. Schutz \& R. Pekrun (Eds.), Emotion in education (pp. 313-331). San Diego, CA: Academic.

Pekrun, R., Elliot, A. J., \& Maier, M. A. (2009). Achievement goals and achievement emotions: testing a model of their joint relations with academic performance. Journal of Educational Psychology, 101, 115-135.

Pekrun, R., Goetz, T., Daniels, L. M., Stupnisky, R. H., \& Perry, R. P. (2010). Boredom in achievement settings: control-value antecedents and performance consequences of a neglected emotion. Journal of Educational Psychology, 102, 531-549.

Rosenberg, E. L. (1998). Levels of analysis and the organization of affect. Review of General Psychology, 2, 247-270.

Schutz, P. A., \& Pekrun, R. (Eds.). (2007). Emotion in education. San Diego, CA: Academic.

Spielberger, C. D., Anton, W. D., \& Bedell, J. (1976). The nature and treatment of test anxiety. In M. Zuckerman \& C. D. Spielberger (Eds.), Emotions and anxiety: New concepts, methods, and applications (pp. 317-344). Hillsdale, NJ: Erlbaum.

Tellegen, A., Watson, D., \& Clark, L. A. (1999). On the dimensional and hierarchical structure of affect. Psychological Science, 19, 297-309.

Turner, J. E., \& Schallert, D. L. (2001). Expectancy-value relationships of shame reactions and shame resiliency. Journal of Educational Psychology, 93, 320-329.

Watt, J. D., \& Vodanovich, S. J. (1999). Boredom proneness and psychosocial development. Journal of Psychology: Interdisciplinary and Applied, 133, 303-314.

Wine, J. D. (1971). Test anxiety and the direction of attention. Psychological Bulletin, 76, 92-104.

Zeidner, M. (1998). Test anxiety: The state of the art. New York: Plenum.

\section{Empathetic Agents}

- Virtual Change Agents

\section{Empathic Understanding}

Empathic understanding is a continuing desire and a conscious decision to understand the other person from his/her own point of view and context. It is a sensitive empathy with each of the other persons' feelings and communications as they seem to him/her at that moment. Acceptance does not mean much until it involves understanding. There is implied here a freedom to explore oneself at both conscious and unconscious levels.

\section{Empathy}

Altruism and Health

\section{Empirically Based Theory of Knowledge}

- Naturalistic Epistemology

\section{Empirically Supported Methods of Instruction}

Behavioral Approaches to Instruction

\section{Empowering Health Learning for the Elderly (EHLE)}

LAURA THOROGOOD

EHLE Project, Padua, Italy

\section{Synonyms}

Communication training for health professionals; Health Learning and the elderly; Health professionals and training; Older people and health questions; Senior citizens and health learning; Teaching health professionals

\section{Definition}

Older people can be empowered to improve their health behavior through education and support from 
medical (or social care) professionals. Teaching these professionals more effective communication on five key topics enables them to empower older people more effectively, and thus improve their mental and physical well-being.

\section{Theoretical Background}

The population of older people, particularly in Western Europe and the USA, is increasing. Not only is it increasing in terms of numbers, but also as a proportion of the overall population. These older people are also living for longer. These three factors mean that while the demand for care and services is growing, the supply of people and money to provide these is shrinking.

Many projects and programs have been implemented to sustain these demographic changes and mitigate their negative effects, allowing older people not only to live longer but to live in better health and in an active nondisabled condition (Beswick et al. 2008). Several methods have been tested by health professionals to improve physical function and independent living among older persons (Fries 2002), and it has been argued that improving communication between patient and health professionals is as important as developing new drugs for major diseases (Fries 2002). In fact, there is evidence to show that education is a key factor in determining health disparities and health outcome among older persons (Guralnik et al. 1993). One method of improving levels of education among older persons is to train health professionals to communicate more effectively with their older patient and to empower them through (a) the effective transmission of relevant information and (b) through the use of different communication techniques to inform understanding of the situation. This was the approach selected by the EHLE Project.

\section{Important Scientific Research and Open Questions}

The empowerment of the older population through their health practitioner (chiefly nurses, doctors, and pharmacists, although other health professionals have been reached) carries four main benefits:

- Health professionals learn to communicate complex knowledge in a way that is relevant and accessible for older people, meaning their work is more effective and their relationship with the patient is more successful.

- Over the course of a working day one health professional can come into contact with many older people, and thus this type of training should improve the health and well-being of more older people than training older people directly.

- Older people are educated about the risks and benefits of their health choices and informed about healthy alternatives and thus are able to take an active role in the upkeep and improvement of their health.

- The learning involved is a stimulus to the mind and respects the older person as one of the best experts on their own health and context.

Disadvantages include: potential reluctance of health professionals to attend training; need to follow up or provide top-up training in order to cement techniques learned (and ensure compliance); effectiveness of this system is weakened if the older person does not see the same practitioner on a regular basis (including pharmacists in the health professionals trained can help avoid this problem).

Having identified the target group and the beneficiaries, we surveyed the beneficiaries in order to find out the key issues that affect their health. We asked questions about both physical health and mental wellbeing, and our survey data showed that the latter was in fact directly associated with health behaviors. Those with a more favorable lifestyle profile showed higher rates of emotional well-being. This confirmed the association between healthy lifestyles and positive emotional status and we hypothesized that any intervention to improve physical health would have a positive indirect effect on their emotional status. The questionnaires revealed that those older people who reported higher levels of unhappiness, anxiety, and depressive symptoms were also more likely to have the less favorable lifestyle profile, such as being sedentary, smoking or having a higher BMI.

Working with health professionals and communications experts we identified five key areas to train health professionals in order to improve health and well-being for older people in particular: prescription and use of medicines, physical activity, emotional health, tobacco and alcohol use, and nutrition. 
We also created a sixth module on communication techniques.

- The first five "information" modules are designed specifically to inform health professionals about key issues for older people in these areas, and to give health professionals simple ways to express or pass on this knowledge.

- The sixth "techniques" module gives health professionals tools and techniques for effectively eliciting key information from older people, effectively communicating key information to older people, and effectively empowering older people to use this information to make and maintain better health choices.

These modules provide a sample training course to teach health professionals (the target group) in order to benefit the health of older people (the beneficiaries). The six modules each have a theoretical and a practical part; the practical part allows health professionals to practice what they learnt in the theoretical part, to evaluate their own and others' learning, and to discuss the results.

\section{Cross-References}

- Communication and Learning

- Health Learning

- Motivation, Volition, and Performance

- Persuasion and Learning

- Psychology of Learning

\section{References}

Beswick, A. D., Rees, K., Dieppe, P., Ayis, S., Gooberman-Hill, R., Horwood, J., \& Ebrahim, S. (2008). Complex interventions to improve physical function and maintain independent living in older people: A systematic review and meta-analysis. Lancet, 371(9614), 725-735.

Fries, J. F. (2002). Aging, natural death, and the compression of morbidity. 1980. Bulletin of the World Health Organization, 80(3), 245-250.

Guralnik, J. M., Land, K. C., Blazer, D., Fillenbaum, G. G., \& Branch, L. G. (1993). Educational status and active life expectancy among older blacks and whites. The New England Journal of Medicine, 329, 110 (www.ehle-project.eu).

\section{Enactive or Action Schemas}

- Sensorimotor Schema

\section{Enactive Schema}

Action Schemas

\section{Encoding}

Infant Learning and Memory

\section{Encouragement}

Incentives and Student Learning

\section{Enculturation and Acculturation}

DAVID H. KIRSHNER ${ }^{1}$, LINGQI MENG ${ }^{2}$

${ }^{1}$ Department of Educational Theory, Policy, and

Practice, Louisiana State University, Baton Rouge,

LA, USA

${ }^{2}$ Penn State Berks, Reading, PA, USA

\section{Synonyms}

Assimilation; Inculcation; Socialization

\section{Definition}

Enculturation and acculturation refer broadly to the processes whereby newcomers come to participate in the normative practices of a cultural community. In relation to each other, enculturation generally signals the case in which the newcomer is an immature member of the cultural community into which she or he is being socialized (e.g., a child); acculturation signals the case in which the newcomer is not a member of the cultural community (e.g., an immigrant). These processes variously include instruction by agents of the culture (e.g., parental scolding), conscious emulation, and unconscious adaptation. Whereas typically the focus of interest is the changing newcomer, enculturation and acculturation have been recognized as involving "reciprocal accommodation" (Simons 1901) in 
which both newcomer and cultural community adapt to one another. Change in the cultural community is particularly evident when the newcomer is part of a community of newcomers sharing a common cultural background. Indeed, acculturation is summarized in Rudmin's (2003) extensive review as "the processes by which individuals, families, communities, and societies react to inter-cultural contact," thereby enabling a focus on the individual, the society, or on intermediate levels of social organization.

\section{Theoretical Background}

Enculturation and acculturation are used in many social sciences discourses including sociology, psychology, anthropology, political science, education, and linguistics; however, in keeping with the root word "culture" the home discipline is anthropology. The first known use of "acculturation" was in a report by J.W. Powell 1880 to the Bureau of American Ethnography on changes in Native American languages wrought by contact with speakers of European languages. In his presidential address to the Anthropological Society of Washington 3 years later, Powell (1883) characterized acculturation as the psychological changes induced by cross-cultural imitation.

Scholarly interest in acculturation has its roots in the very practical concerns and problems that ensue from intermixing of cultural groups. For instance, in his dialogs (Laws), Plato recommended that foreign visitors be kept outside the walls of the city to minimize cultural contamination. Indeed, the most consistent and enduring strand of usage of acculturation concerns the patterns of response that are possible when a minority member of a First-culture intermixes with a majority Contact culture. John Berry developed a well-known taxonomy of four acculturation outcomes depending on whether the minority member does $(+\mathrm{F})$ or does not $(-\mathrm{F})$ identify with the first-culture, and whether she or he does $(+\mathrm{C})$ or does not $(-\mathrm{C})$ identify with the contact culture: assimilation $[-\mathrm{F}+\mathrm{C}]$, rejection $[+\mathrm{F}-\mathrm{C}]$, integration $[+\mathrm{F}+\mathrm{C}]$, or deculturation $[-\mathrm{F}-\mathrm{C}]$. Berry (1980) refined his taxonomy depending on whether members of the minority are granted the right $(+\mathrm{R})$ or denied the right $(-\mathrm{R})$ by the majority culture to freely determine their affiliation. This defines an eightfold taxonomy of acculturation possibilities: assimilation by melting pot $[-\mathrm{F}+\mathrm{C}$ $+\mathrm{R}]$ or by pressure cooker $[-\mathrm{F}+\mathrm{C}-\mathrm{R}]$; rejection by withdrawal $[+\mathrm{F}-\mathrm{C}+\mathrm{R}]$ or by segregation $[+\mathrm{F}-\mathrm{C}-\mathrm{R}]$; integration by multiculturalism $[+\mathrm{F}+\mathrm{C}+\mathrm{R}]$ or by pluralism $[+\mathrm{F}+\mathrm{C}-\mathrm{R}]$; and deculturation by marginality $[-\mathrm{F}-\mathrm{C}+\mathrm{R}]$ or by ethnocide $[-\mathrm{F}-\mathrm{C}-\mathrm{R}]$. This, and other classificatory schemes (see Rudmin 2003), have usefully served as frameworks for articulating tensions and adaptations of minority cultures with respect to a majority culture.

Although acculturation has tended to be used with respect to intergroup adaptation, the term also has encompassed intracultural adaptation. Enculturation, the process of coming to participate in the normative practices of one's native culture, only arose as a distinct construct in the mid-1950s (Herskovits 1955). To this day, usage is inconsistent; some authors use acculturation inclusively to embrace both inter- and intracultural adaptation, and others extend enculturation as also including intercultural adaptation. The two constructs are in an ongoing process of differentiation.

Whereas conscious and unconscious processes are seen as underlying both enculturation and acculturation, acculturation studies generally have focused on highly salient aspects of intergroup difference. The acculturation processes, therefore, have tended to be mediated by consciously accessible feelings, identity structures, and political affiliations. In contrast, enculturationist processes tend to be less subject to conscious mediation. In social psychology the pioneering research of E. T. Hall (1966) into proxemic relations is characteristic of enculturation interests. Hall identified culture-specific norms for drawing physical perimeters around one's body for various social purposes, for instance, the tendency for natives of France to prefer closer physical proximity for conversation than do Americans. Hall noted the unconscious character of the adaptations within each cultural community, the very existence of proxemic enculturation becoming salient only as a result of differing crosscultural norms.

The emergence of the enculturationist interest in underlying, unconscious processes of adaption parallels the contemporaneous development in linguistics of Noam Chomsky's generative approach which transformed that field from a classificatory science concerned primarily with salient exceptions and irregular forms to an explanatory study of the underlying unconscious regularities of language. But whereas Chomsky single-handedly transformed the study of 
language structure almost overnight, enculturationist interests have had no such champion. As a result, scholarship in anthropology, psychology, sociology, and other social sciences concerned with social development has been much more fragmented and uneven with respect to the primacy given to enculturation. Yet as Kirschner and Martin (2010) document, in the past few decades, a veritable forest of sociocultural theories has grown up in psychology in which "processes, such as the mind and the self, [are viewed] as phenomena that are socioculturally constituted - that is, actually made up within, as opposed to merely facilitated by, culture and society" (p. 1). If anthropological interests follow this trend line, unconscious enculturational processes may well emerge as providing the primary explanatory frameworks, with consciously experienced tensions of acculturation regarded as a special case.

A second pressure on the enculturation/acculturation constructs comes from applicative domains in which notions of cultural adaptation have been appropriated for study of idiocultures including apprenticeship communities and classrooms, as well as the cultures of disciplines and professions. For instance, whereas educators have long been interested in the school as a site of acculturation to the broader society, a recent trend is to recruit enculturation and acculturation processes as pedagogical methods with respect to specific subject matter competencies. In this strategy, disciplinary communities such as those of historiographers, scientists, and mathematicians are regarded as cultures whose normative practices are valued as instructional objectives. The pedagogical strategy is to nurture those practices within the classroom microculture so that they become normative. Thus, for example, inquiry groups in the science classroom may provide students autonomy in determining the problem, developing hypotheses, designing experiments, and/or evaluating results so that the scientific method becomes not a list of steps to be memorized, but a living set of cultural practices. This is not a didactic process in which the teacher explains the practices that students rehearse in their groups. Rather, the teacher supports the emergence of normative cultural practices within the classroom microculture, and students learn through their participation in the emerging culture.

Yackel and Cobb (1996) borrow the construct of "reflexivity" from ethnomethodology to explain the challenges of this kind of pedagogy with respect to sociomathematical norms, the classroom social norms that are specific to disciplinary mathematics culture:

- With regard to sociomathematical norms, what becomes mathematically normative in a classroom is constrained by the current goals, beliefs, suppositions, and assumptions of the classroom participants. At the same time these goals and largely implicit understandings are themselves influenced by what is legitimized as acceptable mathematical activity. It is in this sense that we say sociomathematical norms and goals and beliefs about mathematical activity and learning are reflexively related. (p. 460)

The solution to this problem constitutes the critical expertise of the enculturationist teacher. As Yackel and Cobb (1996) illustrate, through subtleties of attention and encouragement the teacher, over time, exerts considerable influence on the modes of engagement manifest within the classroom microculture. As the valued forms of engagement become more prevalent, they become culturally normative, and hence subject to being learned through the same processes of enculturation and acculturation as function in national cultures. This example illustrates how these key notions of enculturation and acculturation are being productively transferred from national cultures to specialized cultures.

\section{Important Scientific Research and Open Questions}

A third source of tension in the enculturation/acculturation literature comes from the poststructural dissolution of the sovereign individual into disparate subjectivities: "the subject is not constituted by just one cultural discourse, or by a single relationship, but rather by a multiplicity of these sources of 'otherness"' (Kirschner and Martin 2010, p. 9). From this perspective, one's national identity, of particular interest to acculturation researchers, is not an unproblematic piece of a (relatively) static identity structure, but often in open conversation and contest with other identity elements including, prominently, gender, race, and class. This tension is evidenced by a rising chorus of protest to what often is characterized as a reductionist and mechanistic study of social relations that is the received legacy of acculturation scholarship informed by a static psychology of the individual 
(for example, see the March 2009 special issue of International Journal of Intercultural Relations).

Whereas the shifts in usage occasioned by the differentiation of enculturation from acculturation and by their application to specialized cultures have stretched, but invigorated, the anthropological tradition of acculturation research, the poststructural shift poses a more profound challenge to the legitimacy of a tradition rooted in notions of monolithic cultures intersecting. As Kirschner and Martin (2010) note:

- [l]n a world typified by increasing globalization and intercultural engagement, some sociocultural psychologists (e.g., Bhatia and Hermans and Salgado. . .) have begun to theorize ways in which different cultural selves (or at least self positions), and dialogues among them, now often exist within the same person. Bhatia even points toward a radical retheorizing of concepts such as "culture, identity, diversity, and difference" based on this new understanding of the emerging global context. (p. 9)

Nonetheless, "these scholars continue to assert the possibility of a substantial selfhood, possessed of an agency that matters, even within all of this multicultural multiplicity and fluidity. Where some others worry about fragmentation and displacement, they see exciting possibilities for truly transformative personal/cultural symbioses." (Kirschner and Martin 2010, p. 10).

We have every reason to expect the vigorous tradition of research in enculturation and acculturation to adapt and thrive.

\section{Cross-References}

- Anthropology of Learning and Cognitions

- Cultural Learning

Identity and Learning

- Intercultural Learning

- Learning (and Evolution) of Social Norms

- Learning in the Social Context

- Microculture of Learning Environments

- Self-Adaptation of Cultural Learning Parameters

- Social Construction of Learning

\section{References}

Berry, J. W. (1980). Acculturation as varieties of adaptation. In A. M. Padilla (Ed.), Acculturation: Theory, models, and some new findings (pp. 9-25). Boulder: Westview.
Hall, E. T. (1966). The hidden dimension. New York: Doubleday. Herskovits, M. J. (1955). Cultural anthropology: An abridged revision of man and his works. New York: Knopf.

Kirschner, S. R., \& Martin, J. (2010). The sociocultural turn in psychology: An introduction and an invitation. In S. R. Kirschner \& J. Martin (Eds.), The sociocultural turn in psychology: The contextual emergence of mind and self (pp. 1-27). New York: Columbia University Press.

Powell, J. W. (1880). Introduction to the study of Indian languages (2nd ed.). Washington, DC: U.S. Government Printing Office.

Powell, J. W. (1883). Human evolution: Annual address of the President, J. W. Powell, delivered November 6, 1883. Transactions of the Anthropological Society of Washington, 2, 176-208.

Rudmin, F. W. (2003). Catalogue of acculturation constructs: Descriptions of 126 taxonomies, 1918-2003. In W. J. Lonner, D. L. Dinnel, S. A. Hayes, \& D. N. Sattler (Eds.), Online readings in psychology and culture (Unit 8, Chap. 8). Bellingham: Center for Cross-Cultural Research, Western Washington University. (http://www.wwu.edu/ culture).

Simons, S. (1901). Social assimilation. Parts I, II, III, IV \& V. American Journal of Sociology, 6, 790-822; 7, 53-79, 234-248, 386-404, 539-556.

Yackel, E., \& Cobb, P. (1996). Sociomathematical norms, argumentation, and autonomy in mathematics. Journal for Research in Mathematics Education, 27, 458-477.

\section{Endogenous Opioids in Fear Learning}

Gavan P. McNally, Sindy Cole

School of Psychology, University of New South Wales, Sydney, NSW, Australia

\section{Synonyms}

Endorphin; Enkephalin; Fear conditioning

\section{Definition}

The endogenous opioid peptides are a family of neuropeptides which bind to four receptors: $\mu-, \kappa_{-}^{-}, \delta$-, and ORL receptors. Both the opioid peptides, and their receptors, are widely distributed throughout the mammalian central nervous system with especially high concentrations in nervous system regions implicated in sensation, emotion, motivation, affect, and memory. As inhibitory neuropeptide transmitters, they fine-tune neurotransmission across a wide range of neuronal circuits, setting thresholds or upper limits. 
Fear learning refers to the behavioral paradigm of Pavlovian fear conditioning. In Pavlovian fear conditioning a neutral conditioned stimulus (CS) that is paired with an aversive unconditioned stimulus (US) such as footshock or loud noise comes to elicit conditioned fear responses (CRs) such as freezing behavior, potentiated startle, ultrasonic vocalizations, and changes in heart rate and blood pressure.

\section{Theoretical Background}

Fear learning depends on how informative the CS is regarding US occurrence. This notion of informativeness is captured formally by error-correcting learning theories. These theories state that Pavlovian conditioning proceeds as a function of prediction error, or the discrepancy between the actual and expected outcomes of a conditioning trial. Learning to a CS occurs when there is an unexpected US (positive prediction error), loss of fear to a CS occurs when the US is less than expected (negative prediction error) and no learning occurs to a CS when a US is fully predicted by the CS. These theories explain three key features of fear learning.

First, fear learning is negatively accelerated: more fear is learned across the initial CS-US pairings than across later CS-US pairings. This occurs because prediction error is greater at the start of training, when the US is unexpected. Second, fear once acquired can be extinguished: presentations of a fear CS alone, in the absence of the US, cause reductions in fear. This occurs because of negative prediction error: during extinction there is no aversive US so the expected US is greater than the actual US and fear is extinguished or lost. Finally, fear learning to one CS can prevent fear learning to other CSs. This is exemplified by the Kamin blocking paradigm (Kamin 1968). In this paradigm, subjects are first trained to fear CS1 via pairings with an aversive US. Then they receive pairings of CS1 and CS2 in compound followed by the aversive US. Conditioning fails to CS2, despite CS2-US pairings, because predictive error during the second stage of training is low: subjects can predict the US from CS1 and so learning about CS2 is prevented or "blocked."

The endogenous opioids acting through the $\mu$-opioid receptor mediate these three keys features of error-correcting fear learning (McNally 2009). Blockade of endogenous opioids (e.g., via neural or pharmacological manipulations): (1) removes negative acceleration of fear learning thereby augmenting the acquisition of fear learning; (2) prevents the extinction of fear learning; and (3) prevents the Kamin blocking of fear learning. Conversely, augmenting endogenous opioids prevents the acquisition of fear and augments the extinction of fear. These effects are achieved primarily via opioid peptide binding to the $\mu$-opioid receptor. The $\mu$-opioid receptors in a region of the midbrain, the periaqueductal gray, are especially important for these effects. These actions at midbrain $\mu$-opioid receptors determine variations in the effectiveness of the aversive US as a reinforcer or teaching signal for learning. The presentation of a fear CS causes $\mu$-opioid receptor activation in the periaqueductal gray which in turn causes a diminution or reduction in processing of the aversive US. In this way, the actions of opioids prevent expected aversive USs from producing further fear learning. This action is similar to negative feedback (Fanselow 1998).

\section{Important Scientific Research and Open Questions}

There are three important domains of research effort into the roles of opioids and fear learning. The first examines the neuropharmacology and neural mechanisms for endogenous opioid receptor contributions to fear learning. This has included identification of the critical peptide and receptor subtype via traditional neuropharmacological techniques such as microinjections of receptor agonists or antagonists into discrete brain regions, as well as via modern molecular neuroscience techniques such as opioid peptide gene or receptor gene knockout (McNally 2009). The second examines opioid receptor contributions to human fear learning. This has involved studying the effects of opioid receptor antagonists on human fear learning and includes functional neuroimaging of brain activity during fear learning after opioid receptor antagonist administration (Eippert et al. 2008). The third involves clinical translation of these findings to treatment of human anxiety. Endogenous opioids have been found to mediate the therapeutic benefits of exposure and systematic desensitization therapies for simple phobia. A critical question raised by this research is whether augmenting endogenous opioids in humans can help prevent the development of anxiety disorders or serve as pharmacological adjuncts to psychological treatments for anxiety. 


\section{Cross-References}

- Extinction Learning

- Fear Conditioning

- Formal Learning Theory

Learning Not to Fear

\section{References}

Eippert, F., Bingel, U., Schoell, E., Yacubian, J., \& Buchel, C. (2008). Blockade of endogenous opioid neurotransmission enhances acquisition of conditioned fear in humans. Journal of Neuroscience, 28, 5465-5472.

Fanselow, M. S. (1998). Pavlovian conditioning, negative feedback, and blocking: mechanisms that regulate association formation. Neuron, 20, 625-627.

Kamin, L. J. (1968). 'Attention-like' processes in classical conditioning. In M. R. Jones (Ed.), Miami symposium on the prediction of behaviour: Aversive stimulation (pp. 9-33). Miami: University of Miami Press.

McNally, G. P. (2009). The roles of endogenous opioids in fear learning. International Journal of Comparative Psychology, 22, 153-169.

\section{Endorphin}

Endogenous Opioids in Fear Learning

\section{Engaged Learning}

- Joyful Learning

- School Motivation

\section{Engagement}

Alertness and Learning of Individuals with PIMD

- Interactive Learning Techniques

\section{Engagement Enhancement}

Motivation Enhancement

\section{Engagement in Learning}

Robert M. CARINI

Department of Sociology, University of Louisville, Louisville, KY, USA

\section{Synonyms}

Student effort; Student engagement; Time on task

\section{Definition}

Engagement in learning is a nebulous concept, one that has been defined in different ways. One view holds that engagement is "... the time and effort students devote to activities that are empirically linked to desired outcomes... and what institutions do to induce students to participate in these activities" (Kuh 2009, p. 683). Chickering and Gamson (1987) provide a more detailed definition by identifying seven guidelines for engagement in higher education:

- Increase student-faculty interactions both inside and outside the classroom.

- Give prompt feedback on students' work.

- Set high expectations for students by challenging them to put forth their best effort.

- Provide collaborative learning opportunities with other students.

- Provide experiential learning opportunities.

- Maximize students' time on task in curricular pursuits.

- Appreciate diversity in students' talents and learning styles.

Engagement, then, is multidimensional. In addition to those already mentioned, pundits have considered a host of other dimensions: students' attendance; preparedness for class; temporal persistence in schooling; perceptions that the curriculum is interesting and relevant; exposure to diverse ideas/people/experiences; - deep learning approaches; emotional connectedness to their class or school; perception of a supportive - school climate; and enriching cocurricular activities such as science fair participation, community involvement, or student government. Perhaps "engagement can best be understood as a relationship: between the student and school community, the student and school adults, the student and peers, the student and 
instruction, and the student and curriculum" (YazzieMintz 2009, p. 1). What definitions have in common is that greater engagement - whatever its form - encourages student learning and development. While engagement has intuitive, and even popular, appeal, the rationale for its use is grounded in empirical research. With appropriate modification, principles of engagement may be applied to different grade levels, settings outside of formal schooling, and within different cultures.

\section{Theoretical Background}

The underpinnings of student engagement have been in place for decades. While the phrase "student engagement" was not used widely until the late1990s, educators have long sought to maximize students' time on task and increase their motivation to learn. During the late 1970s and 1980s, researchers examining higher education moved to the vanguard of studying the import of student experiences, as they realized that family background characteristics, such as socioeconomic status, are weak predictors of student learning and graduation once students have entered higher education. Further, greater attention to student experiences helped counter complaints that higher education relied too heavily on lecturing, which is often viewed as a disengaging mode of instruction. Initial studies focused on the quality and quantity of student effort (for example, see Pace 1980). Alexander Astin (1932 - present) emphasized that students actively shape their own outcomes in higher education. He posited that learning and developmental outcomes are enhanced if students invest more time and energy in study, school relationships, and activities. These pioneering studies on student experiences would eventually evolve into what is now known as "student engagement."

By 2000, scholars had uncovered a host of contextual factors that support or inhibit engagement, such as instructional style, classroom conditions, school programs, and school climate. In the last decade, research on engagement has increasingly emphasized the transition from secondary to tertiary schooling, first-year experiences in higher education, and specific practices that make particular schools highly engaging for students. Although those who study higher education remain at the forefront of inquiry into engagement, educators at all grade levels apply its principles. Elements of engagement have been applied within a wide variety of disciplines within education, including business, engineering, legal studies, psychology, and sociology.

The premise of engagement is straightforward: a host of outcomes should improve as students put more time, quality effort, and enthusiasm into fruitful learning opportunities. Learners should be exposed to interesting, challenging, and often experiential learning opportunities in a supportive and safe environment. Engagement is widely viewed as a recipe for reversing student boredom and apathy toward schooling. It can be used as a practical lever to improve many student outcomes. Indeed, engagement is often used as a proxy for student learning, particularly when direct measures are unavailable. Engagement complements traditional measures of school quality such as reputation, financial resources, facilities, and faculty's research and awards. Since engagement taps directly into student experiences, it may more directly assess learning opportunities than traditional measures of school quality.

In an era of greater accountability, schools have turned to engagement for purposes of external accreditation, self-reflection, faculty and staff development, marketing, and benchmarking their progress. Students and their parents, teachers, and administrators have also shown enthusiasm for engagement; The National Survey of Student Engagement (NSSE) is a testament to the groundswell of interest. NSSE is administered annually to first-year students and seniors at baccalaureate-granting schools. Students at nearly 1,400 schools in the United States and Canada have completed NSSE over the last decade, and the survey has been adapted for use in a number of countries outside North America (NSSE 2009). Results from NSSE show that some schools have improved their students' engagement over time (NSSE 2009). NSSE has spawned related surveys that tailor their engagement measures to different types of schools, grade levels, and faculty, e.g., the Classroom Survey of Student Engagement, High School Survey of Student Engagement, the Community College Survey of Student Engagement, and the Faculty Survey of Student Engagement. Given its broad-based utility, engagement has become a "buzzword," as well as an important reform movement in education over the last two decades. 


\section{Important Scientific Research and Open Questions}

An impressive number of studies exist on student engagement. One strand has investigated its correlates, i.e., variables that are related to higher or lower engagement. A consistent finding is that females tend to be more engaged in school than males. Further, students in "honors" or "gifted" classes tend to report more engagement than those in regular or lower tracks. Those who are enrolled full time in higher education evince more engagement than part-timers. Students who transfer to a new school tend to have lower initial engagement. Findings like these led to the coining of the phrase "engagement gap," a pattern wherein engagement is higher for one group of students than another (Yazzie-Mintz 2009). For their part, faculty promote engagement via their enthusiasm, use of a variety of instructional and assessment methods, and customization of the curriculum for relevancy to students' lives. In contrast, standardized tests tend to be disengaging for many students. Smaller schools and class sizes, everything else being equal, tend to promote engagement. A school climate that consistently upholds the value of excellence in teaching and learning, while also providing a safe learning milieu, fosters engagement.

Another strand of research has established empirical links between engagement and student outcomes. Greater engagement is connected to a bevy of favorable outcomes, i.e., educational persistence, higher grades, higher scores on critical thinking tests, greater student satisfaction with their education, and more personal and social development. Increasingly, researchers have explored conditional effects, or, in other words, whether the linkages between engagement and its outcomes vary for different groups, subject matter, levels of schooling, types of schools, and community settings. This strand is - in part - fueled by concerns that the benefits of engagement might not extend to nontraditional or disadvantaged students (for example, see Carini et al. 2006; Kuh 2009).

Research in this entire area has been hampered by theoretical and methodological issues, including a lack of consensus on how to define and measure engagement, an overreliance on self-reports instead of objective measures, a paucity of longitudinal data to test causal linkages, and disappointingly weak relationships between engagement and certain student outcomes.
An ongoing issue is whether - or how to - disentangle the relative strengths of dimensions of engagement, i.e., which dimensions wield the most powerful and weakest effects on student outcomes. For instance, peer tutoring appears to be a particularly potent form of collaborative learning.

Despite the volume of research, many important questions remain unanswered. For instance, the engagement "movement" challenges students to make effective use of their non-sleeping hours. Time-use studies suggest that high school and college students face an array of activities that compete for their time outside of formal schooling, including employment, caregiving in the household, commuting, and leisure pursuits. Certain activities such as cocurricular interests and on-campus employment appear to have benign or even positive effects on learning. Yet, time spent in some activities, and under certain conditions, may distract students from academic pursuits. For example, extracurricular leisure, including socializing and relaxation, has largely unknown effects on engagement.

An emphasis on engagement also presses faculty to teach more effectively. In particular, engagement brings attention to the unwritten, but implicit, "disengagement compact" between faculty and students (Kuh 1999), i.e., faculty lower their expectations concerning required student effort - and offer students a reasonable grade - in exchange for less hassle from students, fewer office hours, and more favorable course evaluations. If meaningful change is to occur within - school culture, "disengagement compacts" must be renegotiated. Schools can take the lead by forging cultures that value student achievement and quality of instruction. Indeed, one overlooked pathway to higher student engagement is to boost faculty's engagement in teaching. Further, although effective engagement strategies are well known, some teachers are unsure about how to implement them practically in the classroom.

Work also remains to be done on how engagement may be harnessed to improve learning in novel situations. For instance, the rapid pace of technological change hinders the timeliness of research focused on the implications of new technologies for instruction and learning. For instance, social networking services such as Facebook and Twitter offer exciting opportunities to engage students with technologies that many already use and enjoy. Further, the proliferation of 
online distance education has offered students unprecedented convenience and choice in scheduling their learning. Yet, some wonder if engagement may suffer in wholly online or hybrid courses. Engagement in online or hybrid learning environments may pose special challenges for course design, administration, and choice of instructional technologies. Faculty often struggle with how to develop "community" online, animate the curriculum, and provide quality communication with students. However, online tools such as blogs, wikis, and discussion boards may help counter the possible alienating aspects of online environments. Research has begun to bridge this lacuna by assessing online students' engagement vis-à-vis those of oncampus counterparts, and how engagement can be encouraged online. When enrollment in online courses is voluntary, those who choose online courses likely differ in their motivations. It is an empirical question as to whether those who select online courses may be less receptive to certain dimensions of engagement, such as collaborative learning with peers.

\section{Cross-References}

- Academic Learning Time

- Boredom of Learning

- Climate of Learning

- Collaborative Learning

- Critical Thinking and Learning

- Experiential/Significant Learning

- Measurement of Student Engagement in Learning

- School Climate and Learning

- Styles of Engagement in Learning

\section{References}

Carini, R. M., Kuh, G. D., \& Klein, S. P. (2006). Student engagement and student learning: Testing the linkages. Research in Higher Education, 47(1), 1-32. doi:10.1007/s11162-005-8150-9.

Chickering, A. W., \& Gamson, Z. F. (1987). Seven principles for good practice in undergraduate education. American Association for Higher Education Bulletin, 39(7), 3-7.

Kuh, G. D. (1999). How are we doing?: Tracking the quality of the undergraduate experience, 1960s to the present. The Review of Higher Education, 22(2), 99-120.

Kuh, G. D. (2009). What student affairs professionals need to know about student engagement. Journal of College Student Development, 50(6), 683-706. doi:10.1353/csd.0.0099.

National Survey of Student Engagement. (2009). Assessment for improvement: Tracking student engagement over time-Annual results 2009. National Survey of Student Engagement website.
Retrieved 16 June 2010, from http://nsse.iub.edu/ NSSE_2009_Results/pdf/NSSE_AR_2009.pdf

Pace, C. R. (1980). Measuring the quality of student effort. Current Issues in Higher Education, 2, 10-16.

Yazzie-Mintz, E. (2009). Charting the path from engagement to achievement: A report on the 2009 High School Survey of Student Engagement. High School Survey of Student Engagement website. Retrieved 17 June 2010, from http://ceep.indiana.edu/hssse/ images/HSSSE_2010_Report.pdf

\section{Engagement Intervention}

Motivation Enhancement

\section{Engagement Profile}

Styles of Engagement in Learning

\section{Enhanced Discrimination}

- Effects of Physical Context Change and Perceptual Learning on Generalization

\section{Enkephalin}

- Endogenous Opioids in Fear Learning

\section{Ennui}

Boredom in Learning

\section{Enrichment}

- Imaginative Learning 


\section{Enrolled Home Study}

Homeschooling and Teaching

\section{Entity Theory}

View that intelligence or ability is fixed, stable, and uncontrollable.

\section{Entropy}

A measure of interstimulus variability; two or more identical stimuli on the screen would produce an entropy value of 1.0, and this value would increase as a function of the more different stimuli that are visible.

\section{Environmental Awareness}

Environmental awareness may be understood as conceptual knowledge of problem situtations, while the second approach considers it as closely connected to the actions and system components, where intentions and values play an important role (Pata and Metsalu 2008).

\section{References}

Pata, K., \& Metsalu, E. (2008). Conceptualizing awareness in environmental education: An example of knowing about air-related problems. Science Education International, 19, 41-65.

\section{EPAM}

A cognitive architecture developed by Herbert Simon and Edward Feigenbaum. Both the limits of human cognition (e.g., limited capacity of short-term memory) and the means to assuage these limits (through learning mechanisms) are emphasized. EPAM stands for Elementary Perceiver And Memorizer.

\section{Epigenetic Robotics}

- Cognitive Robotics

- Developmental Robotics

\section{Episodic Future Thinking in Birds}

Planning in Birds

\section{Episodic Learning}

ANDREw M. Nuxoll

Department of Computer Science, University of

Portland, Portland, OR, USA

\section{Synonyms}

Experiential learning; Instance-based learning

\section{Definition}

Episodic learning is the process of storing experiences in one's episodic memory or retrieving that information and using it to improve behavior. Episodic memory (Tulving 1983) is a long-term memory used to store unique events, or episodes, from one's past. For example, you may have a semantic memory of the major events surrounding the 9/11 attack on the World Trade Center. However, you would have an episodic memory of where you were and what you were doing at the time that you first learned of that event.

Episodic learning is a form of experiential learning. It is particularly distinguished by the fact that the content is not filtered or modified as it is learned. Instead, the episode is recorded exactly as it was experienced and stored in such a way that its temporal context, relative to other episodic memories, is evident. When a related stimulus occurs, the previous episode is recalled and used to influence the rememberer's actions.

\section{Theoretical Background}

Awareness of human episodic memory is intuitive and dates well before modern psychology. Endel Tulving 
(1983) was the first to name and define episodic memory on a phenomenological basis. He observed that humans in word-memorization experiments remembered the event of reading each word in a specific context rather than remembering the word itself. Based on these and further observations, Tulving identified several features that distinguished episodic memory from semantic memory and used that evidence to argue that episodic memory was a distinct capability of the human brain. Among these features is that episodic memory is autonoetic (i.e., the remember recalls the episode from his own point of view), temporally indexed and particularly vulnerable to forgetting and false memories.

Perhaps the most convincing evidence of the distinction between episodic and semantic memory comes from observing patients with amnesia who retain the ability to recall commonly used words but are unable to recall or recognize new words they had been introduced to after their injury.

\section{Important Scientific Research and Open Questions}

The brunt of research on episodic learning is focused on the nature of how it functions, particularly in interaction with semantic memory. A central theme of this line of research is: How and when do episodic memories become semantic memories? Studying this intersection is likely to yield insights into how both memories work.

A prevailing concept is that of autobiographical memory (Baddeley et al. 2001), which can be thought of as a combination of episodic and semantic memories. For example, a memory of your high-school graduation may contain particular details mixed with factual knowledge about your high school and other people who were present. It is also possible that all "episodic memories" are forgotten after a period of hours and that anything we remember from the more distant past is actually a semantic memory.

An open issue that has remained since Tulving's initial work is whether episodic learning is a phenomenon unique to humans or present (if less developed) in other creatures. There is evidence that supports both claims. Arguments for the former hypothesis focus upon the autonoetic property of episodic memory. Research with primates supports the latter hypothesis, and some research $(\downarrow$ Episodic-Like
Memory in Food Caching Birds) indicates that even non-mammals may have episodic memory.

"Flashbulb memory" (Tulving and Craik 2000) is the popular name for the observation that humans tend to record an exceptional amount of detail during acutely emotional situations. Considerable research has been invested into studying this phenomenon because of possible insights into the episodic learning mechanism. However, there is other work that suggests that there is no additional detail in flashbulb memories and that humans merely believe they remember more because of the emotions associated with the remembered experience.

The concept of prospective memory (McDaniel and Einstein 2007) is our ability to recall a particular memory when a relevant context arises. The classic example is remembering (or not!) to pick up milk from the store on your way home. Studies of this phenomenon may yield some insights on the nature of episodic learning.

In the field of neuroscience, researchers are trying to define what parts of the brain are involved in episodic learning and what role they play in encoding, storage, and retrieval. The predominantly accepted outcome from PET and fMRI studies is that episodic learning occurs in the hippocampus (Tulving and Craik 2000). There is also strong evidence that episodic memories are stored in the prefrontal cortex but alternative or additional ideas have been proposed.

More recently, episodic memory has received attention in the field of cognitive modeling. Symbolic models are few and have focused on modeling human behavior in memory intensive tasks.

Connectionist models of episodic learning, attempt to mimic a human's ability to retrieve a memory based upon a particular memory cue (Polk and Seifert 2002). One of the most striking is the Hopfield network which has the ability to learn multiple patterns and, when presented with a cue (a new pattern or a portion of a pattern it has learned), it is able to retrieve the closest matching pattern. Recurrent artificial neural networks have also been used to model the behavior of the hippocampus and to implicitly store temporal information with memories, particularly with regard to learning a sequence of actions.

In the field of artificial intelligence, most AI agents lack the ability to record or recall their past experiences. Such profound amnesia is a rare and very severe handicap for humans, which raises the concern that AI 
agents may suffer from the same handicap. That observation has led to investigation of precisely what human capabilities depend upon episodic learning and how that functionality could be implemented with a computer for the benefit of AI agents. Major functionality that has been observed to date includes: predicting future situations based upon the past, forgetting (as a way to improve performance), explaining past behavior for the benefit of other agents (human or artificial), reviewing past experiences to relearn proper behavior in light of new information, constructing and maintaining long-term plans, and distinguishing novel from routine.

Closely tied to episodic learning for AI agents is the problem of creating an effective artificial episodic memory system to support it. Two related, important areas that seem central to this effort are generalization and retrieval. Generalization is the term applied to extracting semantic facts from regularities in episodic memories. Retrieval is the process of selecting an episode that best matches a given memory cue. This process requires identifying the features of the cue that are "most important" in the context of the agent's current task. For example, if you are asked to recall the last time you ate a candy bar on an airplane, you implicitly know that "being on an airplane" is the more important part of the cue. As a result, if you fail to retrieve an exactly matching memory you're more likely to relate a story of eating fruit on an airplane than eating a candy bar at a movie theater. Recognizing what's important in this manner may be related to generalization. Performing complex retrievals of this type and doing so efficiently remains an open problem.

\section{Cross-References}

- Conditions Affecting the Revelation Effect for Autobiographical Learning and Memory

- Episodic-Like Memory in Food Caching Birds

- Memory Consolidation and Reconsolidation

- Semantic Memory in Profound Amnesia

\section{References}

Baddeley, A., Conway, M., \& Aggleton, J. (Eds.). (2001). Episodic memory: New directions in research. New York: Oxford University Press.

McDaniel, M. A., \& Einstein, G. O. (2007). Prospective memory: An overview and synthesis of an emerging field. Thousand Oaks: Sage.

Polk, T. A., \& Seifert, C. M. (Eds.). (2002). Cognitive modeling. Cambridge, MA: MIT Press.
Tulving, E. (1983). Elements of episodic memory. New York: Oxford University Press.

Tulving, E., \& Craik, F. I. M. (Eds.). (2000). The Oxford handbook of memory. New York: Oxford University Press.

\section{Episodic Memory}

Memory for specific events, places, or experiences that can be explicitly stated. For example, your memory for your trip to France may be comprised of many different episodic memories: getting off the plane, having lunch at the Eiffel Tower, and so forth.

\section{Cross-References}

- Memory for "What," "Where," and "When" Information in Animals

- Prospective and Retrospective Learning in Mild Alzheimer's Disease

\section{Episodic-Like Memory}

- Memory for "What," "Where," and "When" Information in Animals

\section{Episodic-Like Memory in Food-Caching Birds}

\section{Caroline R. Raby, Nicola S. Clayton}

Department of Experimental Psychology, University of Cambridge, Cambridge, UK

\section{Synonyms}

Event memory; Remembering the past; Retrospective cognition

\section{Definition}

Episodic-like memory in birds is the ability to remember a unique event. Human episodic memory is characterized by the feeling of having personally experienced a past event. This contrasts with semantic 
memory, which is knowledge of an event in the past without a sense of having personally experienced it. Knowing that you were born is semantic because you are unlikely to remember it happening. Remembering your first day at school is episodic. As distinguishing an episodic memory from semantic knowledge in humans depends on feelings which can only be described using language, evidence that birds can remember a specific past event is designated "episodic-like."

\section{Theoretical Background}

Some birds have been shown to have what, where, when (www) memory, that is they remember what happened, where and when. If the what, where, and when are integrated, this is sufficient to distinguish an event from all others as no other event could have the same what, where, and when.

Episodic memory in humans was originally defined as www memory but this definition was subsequently modified as it is possible to have semantic knowledge comprising www and episodic memories that consist of what but neither where nor when beyond a sense of pastness. Increasing emphasis has been placed on the phenomenological sense of "reexperiencing the past" dubbed mental time travel (MTT) (Suddendorf and Corballis 1997; Tulving 1985).

As it is not possible to enter an animal's mind, all evidence for cognitive processes must be deduced from their behavior. Clayton and colleagues suggested a number of behavioral criteria for demonstrating episodic memory in animals which do not include the requirement for consciousness of oneself in a different time. In recognition of this, they called this "episodic-like" memory (Clayton and Dickinson 1998). These criteria are:

Content: recollecting what happened, where, and when on the basis of a specific past experience

Structure: forming an integrated "what-where-when" representation

Flexibility: flexible deployment of the information available from the event in new situations

Episodic memories are temporally unique. If knowledge of "what," "where," and "when" can be demonstrated to be bound together in an integrated representation so that retrieving one feature of the episode automatically retrieves the other features, this meets the criteria of structure and content. Episodic memory is a form of declarative memory and as such must also be capable of flexible deployment in novel situations in contrast to procedural memory which is inaccessible to conscious recall and thought to be situation specific and inflexible. Clayton and colleagues argued that it is the "when" component that is the crucial aspect in binding an event together in the kind of episodic recall shown by scrub-jays when they are discriminating between caching events.

However, Eacott and colleagues have proposed that in episodic memory "when" serves as no more than an occasion setter to distinguish one experience from another similar experience. They suggest that episodic memory in animals is better defined as "what-wherewhich" and that the temporal dimension is only one of a number of possible occasion setters. If episodic memory binds components of a specific event together, then, as long as it can be demonstrated that more than one feature of an event has been retrieved in an integrated form, this is a good indicator of an episodic memory (Eacott and Norman 2004).

Other researchers argue that even an integrated www memory which is used flexibly does not necessarily bear any relation to human episodic memory. Firstly there is to date no evidence than an animal has a sense of self which it can project to another time. In addition an event A could cause a cognitive change $B$ that effects behavior $\mathrm{C}$ without $\mathrm{B}$ carrying any information about $\mathrm{A}$.

It is possible that some birds and other animals have "event memory," an evolutionary forerunner of human episodic cognitive abilities that allow them to act on specific past events and take actions for the future but that do not necessarily comprise the experience of mental time travel or of autonoesis.

\section{Important Scientific Research and Open Questions}

Clayton and Dickinson (1998) took the view that ecological relevance was an important consideration in setting tasks to measure episodic memory. Scrubjays cache both perishable and unperishable foods in the wild. They recover perishable foods after short time periods but may leave unperishable foods for weeks or months before recovery. It is reasonable to assume therefore that they have evolved the ability to remember not only what they have cached and where but when. 
Scrub-jays not only remember where they have cached items but also what they have cached in a particular place and when they cached it. If they are given two foods to cache, one of which does not decay and another, favorite, food which they learn decays after a certain amount of time has passed, they will preferentially go to locations in which they have stored the favorite food if they are allowed to recover it before the time after which they have learnt it will be degraded has passed. However, after the length of time in which they have learnt that the favorite food decays has passed, they recover instead the less favored food that does not decay (Clayton and Dickinson 1998). They can also distinguish between two foods one of which is unpalatable a short interval after caching but palatable after a long interval while the other is palatable after a short interval but not after a long interval. Allowed to cache both foods, the jays adjust their recovery behavior to search preferentially for the palatable food after the appropriate delay. There is a considerable amount of evidence that these are integrated memories which can be updated and generalized over situations.

In further experiments, Clayton and Dickinson demonstrated that scrub-jays not only encode information about the type of food they store in cache sites but, given the opportunity to recover items, they can subsequently remember not only which sites have been depleted but also which type of food item they have already recovered. This could be useful in assuring the consumption of a balanced diet in natural conditions. That they show a propensity to balance the foods they eat is shown by several experiments in which the birds show specific satiety, that is to say that if they are prefed on a particular food they show a preference for consuming a different food when subsequently given the choice of the food they have just been eating and a different food. Scrub-jays also alter their caching decisions depending on what they find when they recover their caches

Scrub-jays meet the behavioral criteria for episodic memory proposed by Clayton and Dickinson (Clayton and Dickinson 1998). The jays can remember what they have cached, where and when, and so fulfill the content criterion for episodic-like memory. The fact that they are able to distinguish between memories in which the "what" and "where" are the same but the "when" is different is also evidence that the memory occurs in an integrated representation and so fulfills the structure criterion. Building on the scrub-jay work, other researchers have tested www memory in different birds. Magpies can also remember what they have stored, where and when. They were given pellets of egg dyed red or blue. If they returned to the caching site on the same day, one color of pellets was replaced with wooden beads of a similar size or color. If they returned the next day, the other color of pellets had been replaced with wooden beads. They quickly learnt to vary their searching behavior to look for the food pellets that had not been replaced with beads depending on the retention interval since caching (Zinkivskay et al. 2009). Black-capped chickadees also show www memory but the memory for when depended on the nature of the task with more natural conditions using trees in an aviary eliciting better results than experiments conducted in the birds' home cages. Pigeons, by contrast, seem only to be able to remember the what, where, and when of a single event. The cause of this limitation appears to be an inability to integrate what happened, where and when so that an individual memory can be distinguished from other similar memories It has been suggested that, because the scrub-jays have repeated trials, their responses may be based on semantic knowledge or rule learning and that an essential element of demonstrating episodic memory in animals is that the question that they are asked is unexpected. This excludes the possibility of any form of rule learning. The argument here is that if a question is expected then the answer can be learned, as it were by rote, at the time of encoding. In order to demonstrate that an animal has retrieved the memory of a specific past event it is necessary that, at the time of encoding information, there is no expectation that the information will need to be retrieved. Consider the question of being asked what you ate for breakfast today. If you are expecting to be asked the question, you can encode an answer when you eat breakfast and do not actually have to remember the event itself when the expected question is asked, only the prepared answer. If, however, the question is unexpected you must think back to breakfast time in order to episodically retrieve the wanted information (Zentall et al. 2008).

Zentall demonstrated that pigeons are able to report the location that they recently pecked when 
unexpectedly asked to do so. Zentall trained pigeons to report the location that they had just pecked. They were then trained on a conditional discrimination task to associate different colored samples with differently oriented comparisons (e.g., a blue key with a key with horizontal lines). Once they had learnt this task, the pigeons would suddenly after a $2 \mathrm{~s}$ delay at the end of a trial be presented with the cue to report which key they had just pecked. Zentall argues that the pigeons must have used episodic memory to answer this question because they had no opportunity to acquire semantic or rule-based memory. Critics have suggested that given the short timescale over which these experiments operated the use of working memory cannot be excluded but the use of working memory does not necessarily exclude episodic components (Zentall et al. 2008).

Research to date has been on a small number of birds in very few species. While some birds can clearly act on specific past events, it remains an open question as to the precise cognitive processes that enable this behavior and in particular whether birds consciously recall a past event that they have experienced.

\section{Cross-References}

- Planning in Birds

\section{References}

Clayton, N. S., \& Dickinson, A. (1998). Episodic-like memory during cache recovery by scrub jays. Nature, 395(6699), 272-274.

Eacott, M. J., \& Norman, G. (2004). Integrated memory for object, place and context in rats: A possible model of episodic-like memory? The Journal of Neuroscience, 24(8), 1948-1953.

Suddendorf, T., \& Corballis, M. C. (1997). Mental time travel and the evolution of the human mind. Genetic Social And General Psychology Monographs, 123(2), 133-167.

Tulving, E. (1985). Memory \& consciousness. Canadian Psychology, 26(1), 1-12.

Zentall, T. R., Singer, R. A., \& Stagner, J. P. (2008). Episodic-like memory: Pigeons can report location pecked when unexpectedly asked. Behavioural Processes, 79, 93-98.

Zinkivskay, A., Nazir, F., \& Smulders, T. V. (2009). What-where-when memory in magpies (Pica pica). Animal Cognition, 12(1), 119-125.

\section{Epistemic Beliefs}

- Beliefs About Learning

\section{Epistemic Curiosity}

\author{
JoRDAN A. LiTMAN
}

Psychology Department, University of South Florida, Saint Petersburg, FL, USA

\section{Synonyms}

Cognitive curiosity; Intellectual curiosity; Scientific curiosity

\section{Definition}

Epistemic curiosity is the desire to obtain new knowledge (e.g., concepts, ideas, and facts) expected to stimulate intellectual interest (I-type) or eliminate conditions of informational deprivation (D-type). I-type epistemic curiosity appears to be maximally activated when individuals recognize opportunities to discover something completely new, whereas D-type epistemic curiosity is optimally stimulated when people lack specific pieces of information they wish to incorporate into an existing knowledge-set. Once activated, the degree to which each type of epistemic curiosity is experienced and behaviorally expressed has been found to vary according to individual differences in I- and D-type epistemic curiosity personality traits (Litman 2008).

I- and D-type epistemic curiosity each corresponds to different orientations toward seeking new information. I-type epistemic curiosity involves focusing on the inherent enjoyment of new discoveries, and motivates broadly seeking new information in order to stimulate positive affect. D-type epistemic curiosity reflects an unsatisfied need-like state that motivates seeking knowledge capable of reducing undesirable states of uncertainty about specific unknowns. D-type epistemic curiosity appears to be a stronger motive for knowledge seeking than I-type epistemic curiosity, and is associated with higher levels of state-curiosity and a greater degree of subsequent information seeking behavior as compared to I-type epistemic curiosity (Litman et al. 2005).

I-type epistemic curiosity is a purely intrinsic motive to obtain new knowledge, whereas D-type epistemic curiosity may be conceptualized as an "integrated" motive that involves both the intrinsic pleasure of learning as well as extrinsically regulated concerns about the accuracy or fit of newly gathered information 
(Litman 2008). In academic settings, I-type epistemic curiosity is associated with developing masteryoriented learning goals, the achievement of which leads to increased interest and engagement (Litman 2008). D-type epistemic curiosity is also involved in mastery-oriented learning, but is more strongly associated with setting performance-oriented learning goals, for which the correctness and relevance of newly acquired knowledge is critical to goal achievement. In the workplace, I-type epistemic curiosity is associated with being motivated to develop new skills, whereas D-type epistemic curiosity motivates identifying and solving specific problems (Litman et al. 2010).

\section{Theoretical Background}

The concept of epistemic curiosity as an emotionalmotivational state was first explored by Berlyne (1954), who viewed this construct as a uniquely human desire to know aroused by novel questions or complex ideas that pointed to gaps in one's knowledge. According to Berlyne, epistemic curiosity motivated exploration aimed at the acquisition of new knowledge through observation, thinking, and asking questions. He explicitly differentiated between epistemic curiosity, which motivated the pursuit of knowledge, and seeking stimulation from sensations and perceptions, which he considered to be a common motive of both animals and humans.

Day (1971) later expanded on Berlyne's work, and theorized that epistemic (as well as sensory-perceptual) curiosity corresponded to states of situational interest influenced by individual differences in intrinsic motivation as an aspect of personality. In keeping with Berlyne's theories of epistemic exploration, Day developed measures to assess dispositional tendencies to take pleasure in seeking out both specific and diverse forms of novel or complex stimulation.

Consistent with Berlyne, Loewenstein (1994) also hypothesized that epistemic curiosity was aroused by specific gaps in one's knowledge. He proposed that the degree to which epistemic curiosity was activated depended directly upon an individual's metacognitive awareness of the extent to which he or she had relevant knowledge stored in memory. However, Loewenstein differed with Day's view that epistemic curiosity involved pleasurable states of intrinsic interest, and theorized that subjective experiences of epistemic curiosity primarily reflected uncomfortable states of informational deprivation. According to Loewenstein, seeking out new knowledge is motivated more by "the aversiveness of not possessing the information more than it is by the anticipation of pleasure from obtaining it" (1994, p. 92).

Litman and colleagues reconciled these different views on the nature of epistemic curiosity by examining the circumstances where epistemic curiosity is pure intrinsic motivation and involves feelings of interest (I-type), and when it is oriented toward the elimination of unpleasant conditions of informational deprivation (D-type), and therefore also involves extrinsically regulated concerns about the accuracy and relevance of newly gathered information. Recently, Litman and colleagues developed brief, reliable psychometric instruments for assessing individual differences in the experience and expression of I- and D-type epistemic curiosity (Litman 2008; Litman et al. 2010) that measure tendencies to enjoy learning something completely new (I-type epistemic curiosity scale) as well as the frequency that one feels bothered by a lack of information (D-type epistemic curiosity scale).

Using the I/D epistemic curiosity scales, Litman, Russon and Hutchins (2005) found that I-type epistemic curiosity appears to be maximally aroused when individuals metacognitively determine they have little or no prior knowledge relevant to the information they seek, whereas D-type epistemic curiosity is optimally aroused when individuals feel they have some relevant information already in memory. This suggests that the recognition of knowledge-gaps, as well as the nature and magnitude of the gap, determines whether epistemic curiosity is experienced as feelings of pleasure (Itype) or displeasure (D-type), with the latter found to be a stronger motive to seek out knowledge.

\section{Important Scientific Research and Open Questions}

Several important questions remain regarding the nature of epistemic curiosity, both in terms of its measurement as personality trait and the arousal and satiation of epistemic curiosity as an emotionalmotivational state.

\section{The Measurement of Individual Differences in Epistemic Curiosity as a Personality Trait}

Individual differences in epistemic curiosity are assessed by Litman's (2008) 10-item I/D Epistemic 
Curiosity Scale, which comprises five-item I-type and D-type measures. Although each scale is found to be internally consistent, define different factors and have different correlates, this line of research is relatively new, and further study is needed to elucidate the thoughts, feelings, and behaviors associated with dispositional tendencies to experience and express I- and D-type epistemic curiosity. Accordingly, the degree to which these measures of epistemic curiosity can be meaningfully differentiated from related constructs such as Need for Cognition or Openness requires further investigation (Mussel 2010).

\section{Metacognition and the Arousal of Epistemic Curiosity}

The arousal of I-and D-type epistemic curiosity appears to be associated with different metacognitive states (Litman et al. 2005). However, further research is needed to elucidate why unpleasant feelings are associated with epistemic curiosity when individuals believe they have some prior knowledge in memory; more research is also needed to clarify why state-curiosity is more intense under these circumstances. Loewenstein (1994) suggested that curiosity may intensify when we feel closer to completing a knowledge gap because of learning processes involved in goal attainment; that is, motivation tends to increase in strength as we approach satisfaction of an appetite. Possibly, similar mechanisms underlie self-directed knowledge attainment as well. Another possibility is that the reward-value of new information depends in part on its ability to be incorporated into existing knowledge-sets. These intriguing possibilities need to be explored in future research.

\section{The Satiation of Epistemic Curiosity, Learning, and Experiences of Reward}

On the basis of observable behavior and the selfreported intensity of associated curiosity states, D-type epistemic curiosity appears to be a stronger motive than I-type epistemic curiosity for acquiring knowledge (Litman et al. 2005). However, the underlying reward mechanism for either type of epistemic curiosity remains unknown. Recent research by Kang and colleagues (2009) found evidence of relationships between epistemic curiosity and reward-anticipationrelated brain regions, such as the caudate nucleus and lateral prefrontal cortex. While this research is promising, it is still in its infancy, and much more work needs to be done to clarify the underlying mechanisms of reward associated with the satiation of epistemic curiosity (see also Litman (2005) for recent theories on the underlying physiological mechanisms).

\section{Epistemic Curiosity and Learning over the Life Span}

When seeking out new information, often individuals may make unexpected discoveries - that is, what they actually learn may differ greatly from what was initially expected to be learned. In past research I-type epistemic curiosity has been found to primarily involve broad exploration of wholly new ideas, whereas D-type curiosity appears to be more concerned with the selection of specific pieces of information that will be incorporated into an existing knowledgeframework. Given these different knowledge-seeking goals, we might also expect that I-type epistemic curiosity would be associated with placing greater value on information that diverges from what one already knows or expects, whereas D-type curiosity would be expected to be associated with placing more value on information that converges with expectations. This is a potentially fruitful area for new research on epistemic curiosity that has not been previously explored.

\section{Cross-References}

- Achievement Motivation and Learning

- Adaptation and Learning

- Creativity, Problem Solving and Learning

- Divergent Thinking and Learning

- Epistemic Curiosity

- Interpersonal Curiosity

- Metacognition and Learning

- Motivation and Learning

- Multifaceted Nature of Intrinsic Motivation

- Self-directed Learning and Learner Autonomy

- Understanding Intrinsic and Extrinsic Motivation

\section{References}

Berlyne, D. E. (1954). A theory of human curiosity. British Journal of Psychology, 45, 180-191.

Day, H. (1971). The measurement of specific curiosity. In H. Day, D. Berlyne, \& D. Hunt (Eds.), Intrinsic motivation: A new direction in education (pp. 99-112). Toronto: Holt, Rinehart, and Winston.

Kang, M. J., Hsu, M., Krajbich, I. M., Loewenstein, G., McClure, S. M., Wang, J. T., \& Camerer, C. F. (2009). The wick in the 
candle of learning: Epistemic curiosity activates reward circuitry and enhances memory. Psychological Science, 20, 963-973.

Litman, J. A. (2005). Curiosity and the pleasures of learning: Wanting and liking new information. Cognition and Emotion, 19, 793-814.

Litman, J. A. (2008). Interest and deprivation dimensions of epistemic curiosity. Personality and Individual Differences, 44, 1585-1595.

Litman, J. A., Crowson, H. M., \& Kolinski, K. (2010). Validity of the interest- and deprivation-type epistemic curiosity distinction in non-students. Personality and Individual Differences, 49, 531-536.

Litman, J. A., Hutchins, T. L., \& Russon, R. K. (2005). Epistemic curiosity, feeling-of-knowing, and exploratory behaviour. Cognition and Emotion, 19, 559-582.

Loewenstein, G. (1994). The psychology of curiosity: A review and reinterpretation. Psychological Bulletin, 116, 75-98.

Mussel, P. (2010). Epistemic curiosity and related constructs: Lacking evidence of discriminant validity. Personality and Individual Differences, 49, 506-510.

\section{Epistemological Beliefs}

- Beliefs About Learning

\section{Epistemological Development and Learning}

\author{
Gregory SchraW ${ }^{1}$, Lori Olafson ${ }^{1}$, Michelle \\ VANDERVELDT $^{2}$ \\ ${ }^{1}$ Department of Educational Psychology, University of \\ Nevada-Las Vegas, Las Vegas, NV, USA \\ ${ }^{2}$ Department of Elementary and Bilingual Education, \\ California State University, Fullerton, CA, USA
}

\section{Synonyms}

Beliefs about knowledge

\section{Definition}

Epistemology is the study of beliefs about the origin and acquisition of knowledge (Schommer 1994). This entry focuses on personal epistemology. It is important to distinguish between epistemological beliefs and epistemological world views. Epistemological beliefs consist of specific beliefs about a particular dimension of knowledge such as its certainty, simplicity, origin, or justification. In contrast, epistemological world views consist of a set of beliefs that collectively define one's attitudes about the nature and acquisition of knowledge. An epistemological world view includes all of one's explicit and implicit beliefs, attitudes, and assumptions about the acquisition, structure, representation, development, and application of knowledge (Bendixen and Rule 2004; Olafson and Schraw 2006). Recent research has examined teachers' epistemological world views and how these views are related to teaching practice (Maggioni and Parkinson 2008)

\section{Theoretical Background}

\section{Types of Epistemological Belliefs}

Schommer (1994) described four different and mutually independent epistemological beliefs. The first, simple knowledge refers to the belief that knowledge is discrete and unambiguous. Students who score high on this dimension believe that learning is equivalent to accumulating a vast amount of factual knowledge in an encyclopedic fashion. The second dimension, certain knowledge pertains to the belief that knowledge is constant: Once something is believed to be true, it remains true forever. The third dimension is fixed ability; that is, the belief that one's ability to learn is inborn and cannot be improved through either effort or strategy use. The fourth dimension, quick learning refers to the belief that learning occurs quickly or not at all. Students scoring high on this dimension assume (inappropriately) that limited failure is tantamount to permanent failure. If a problem cannot be solved within $10 \mathrm{~min}$, for example, it will never be solved.

In contrast, Hofer (2001) proposed an alternative four-factor framework and developed an instrument called the Epistemological Beliefs Questionnaire (EBQ) to assess these factors. She proposed four factors, which were subsumed under two general dimensions referred to as the nature of knowing and the process of knowing. The former refers to what knowledge is presumed to be, while the latter refers to how one comes to know and understand knowledge. The nature dimension included two factors called certainty of knowledge (i.e., the degree to which one sees knowledge as fixed versus fluid and changeable) and simplicity of knowledge (i.e., the degree to which knowledge is viewed as individual facts versus complex, interrelated concepts). The process dimension included two factors called source of knowledge (i.e., the extent to which credible knowledge is self- or other generated) and 
justification of knowing (i.e., the rules and criteria that individuals use to evaluate knowledge claims).

Hofer (2001) reported four empirically derived factors that differed somewhat from the four proposed factors described above. Her four observed factors included certainty of knowledge, source of knowledge, justification, and attainment of truth. A simplicity of knowledge factor was not observed, whereas an attainment of truth factor was observed. The attainment of truth factor was interpreted as the extent to which experts can attain deep knowledge (i.e., truth) within their area of expertise. Both the Schommer (1994) and Hofer (2001) instruments are widely used, although there has not been a direct empirical comparison of the two to compare the relative accurcay and validity of either.

\section{Development of Epistemological Beliefs}

Several developmental models of epistemological beliefs have been proposed in which students pass through three ordered stages in the development of beliefs about knowledge (Bendixen and Rule 2004; Hofer 2001). In stage 1, students adopt a dualist perspective, in which knowledge is viewed as either right or wrong. Students in this stage tend to view knowledge as being absolute, universally certain, and accessible only to authorities. In stage 2 , students progress beyond the dualist mode of thinking to a relativist stage, in which knowledge is viewed as uncertain, relative, and personally constructed. Relativists hold the view that knowledge must be evaluated on a personal basis by using the best available evidence. In stage 3 , students construct a contextualist mode of thinking in which knowledge is viewed as constrained by a particular context. Contextualists assume that knowledge will change over time, yet nevertheless possesses local certainty within a particular context in which there is shared set of beliefs and norms.

Research using these three-stage models has reported that development is slow and late-occurring, with significant development at stage 2 and stage 3 occurring between the age of 18 and 22 for college students. A number of researchers have shown that college students move through increasingly sophisticated levels of cognition that do not require epistemic cognition at the lower levels, but require explicit epistemic cognition at the highest levels. Bendixen and
Rule (2004) provided detailed accounts of cognitive mechanisms that underlie epistemological development and belief change, including the important role of education in college students.

\section{Important Scientific Research and Open Questions}

\section{Epistemological Beliefs and Learning}

A variety of studies reported that epistemological beliefs are related to learning and reflective thinking. One consistent finding is that the amount of higher education that students receive is inversely related to their belief in certain knowledge. All other things being equal, the longer students attend college, the more likely they are to believe that knowledge is tentative and subject to personal interpretation. One important implication of this finding is that better educated people may be more willing to adopt a constructivist approach to learning because believing that knowledge is certain should rarely lead one to question the legitimacy of that knowledge. This finding also suggests that encouraging individuals to further their education beyond high school may have a profound effect on their beliefs about knowledge (Olafson and Schraw 2006). A similar effect has been found in research that investigated continuing education in pre- and in-service teachers as well as studies that investigated graduate students' discussions of their epistemological beliefs.

A number of studies conducted during the 1990s reported that beliefs in simple knowledge negatively affected complex problem solving (Hofer 2001). As beliefs in complex, incremental knowledge increased, problem solving improved, although there is some evidence that epistemological beliefs differ across academic disciplines among college undergraduate and graduate students. Students in soft disciplines, such as the humanities, were more likely to believe that knowledge is uncertain than students in hard disciplines, such as physics. Compared with undergraduates, graduate students were more likely to believe that knowledge is uncertain and develops incrementally (they did not believe in quick learning). In addition, epistemological beliefs were related to moral reasoning among adults. Individuals adopting beliefs in complex, incremental knowledge reasoned at a higher level on the Defining Issues Test.

Kuhn (1991) found that epistemological beliefs are related to one's ability to argue persuasively. In 
this study, individuals were classified as an absolutist (one who believes that knowledge is absolutely right or wrong), a multiplist (one who believes that knowledge is completely relative), or an evaluatist (one who believes that knowledge, though relative, is constrained by situational factors such as commonly accepted rules) on the basis of their beliefs about the certainty of knowledge. Evaluatists were more likely than absolutists to provide legitimate evidence in support of an argument. In addition, compared with absolutists, evaluatists generated a greater number of plausible alternative theories and provided better counterarguments.

Together, these studies suggested that beliefs about knowledge and the knowing process affect the way one reasons, how long one persists at a difficult task, the degree to which one is metacognitively engaged in selfregulatory activities, and perhaps what academic discipline one enters (Maggioni and Parkinson 2008). They also indicate that epistemological beliefs are affected by one's home environment and, in particular, by one's educational level. More education seems to translate into a more relativist viewpoint. Ironically, when using objective criteria such as amount and kind of evidence at one's disposal, virtually all studies indicate that contextualist/evaluatist thinking leads to better reasoned conclusions.

\section{Teachers" Epistemological Beliefs}

A number of researchers have argued that teachers' epistemological beliefs influence teaching practices (Maggioni and Parkinson 2008). For example, teachers with more sophisticated epistemological beliefs and world views were more likely to endorse studentcentered instructional practices that emphasize critical reasoning. In addition, teachers with more sophisticated personal epistemologies used a greater number of epistemological moves in their science classrooms, where moves consisted of cognitive activities designed to promote deeper learning and reflection, including generating, constructing, and reconstructing. The success of these moves depended in large part on contextually specific factors such as student knowledge, complexity of activity, and sophistication of students' conceptual understanding; however, epistemological moves were successful when students possessed adequate background knowledge and conceptual understanding. In contrast, teachers with less sophisticated beliefs were more likely to focus on traditional curriculum, student testing, and mastery of basic science concepts.

The development of teachers' epistemological beliefs is another area of study within the field (Maggioni and Parkinson 2008). Several studies have found that pre-service teachers who were enrolled in teacher-training programs based on constructivist, relational, and student-centered pedagogy experienced more growth in sophisticated epistemological beliefs as compared with pre-service teachers in traditional skills-based programs. Researchers also have reported similar findings which suggest that constructivist instruction promotes the development of graduate student teachers at a university (Olafson and Schraw 2006). Teachers in these studies reported a variety of changes during and after the course, but especially changes in epistemological and pedagogical beliefs. One key finding was that teachers were much more likely to adopt constructivist beliefs that emphasized the role of student interactions.

\section{Open Questions}

Additional research in personal epistemology is needed in two areas. One area is understanding the relationship between epistemological beliefs (e.g., certain knowledge) and epistemological world views (e.g., a contextualist perspective). In particular, it is not clear whether some beliefs have a greater impact on worldviews or classroom practices. A second area is to investigate how classroom factors affect the development of students' epistemological beliefs and world views. Recent research suggests that external constraints due to a accountability and testing affect both what and how teachers teach, which in turn, affects student learning (Maggioni and Parkinson 2008; Olafson and Schraw 2006).

\section{Cross-References}

- Belief Formation

- Beliefs About Learning

- Epistemological Beliefs and Learning

- Knowledge Acquisition

- Naturalistic Epistemology

\section{References}

Bendixen, L. D., \& Rule, D. C. (2004). An integrative approach to personal epistemology: A guiding model. Educational Psychologist, 39, 69-80. 
Hofer, B. (2001). Personal epistemology research: Implications for learning and teaching. Educational Psychology Review, 13, 353-384.

Kuhn, D. (1991). The skills of argument. New York: Cambridge University Press.

Maggioni, L., \& Parkinson, M. (2008). The role of teacher epistemic cognition, epistemic beliefs, and calibration in instruction. Educational Psychology Review, 20(4), 445-461.

Olafson, L. J., \& Schraw, G. (2006). Teachers' beliefs and practices within and across domains. International Journal of Educational Research, 45, 71-84.

Schommer, M. (1994). Synthesizing epistemological belief research: Tentative understandings and provocative confusions. Educational Psychology Review, 6, 293-320.

\section{Epistemological State}

- Belief Formation

\section{Epistemologies}

Beliefs About Language Learning

\section{Epistemology}

Epistemology is the branch of philosophy that deals with the nature of knowledge and truth, encompassing the study of the origin, nature, and limits of human knowledge.

\section{Epistemology and Learning}

- Philosophy of Learning

\section{Epistemology and Learning in Medieval Philosophy}

\author{
Norbert M. SeeL \\ Department of Education, University of Freiburg, \\ Freiburg, Germany
}

\section{Synonyms}

Scholasticism

\section{Definition}

In accordance with the basic assumption that epistemology considers the genesis and the nature of knowledge and includes learning (Ernest 1995), the central question of philosophy in general is not whether learning is affected by epistemology, but how. Centering on the question on how humans come to know what they know, the various epochs of philosophy came to different answers to this question at different times. This entry focuses on the particular view of medieval philosophy on learning and epistemology.

Medieval philosophy is the philosophy of Europe in the course of the so-called Middle Ages, the period extending from the end of the Roman Empire in the fifth century to the Renaissance at the end of the fifteenth century. These 1,000 years can be characterized, on the one hand, by a strong reference to ancient philosophy, and, on the other hand, by addressing theological issues aimed at the integration of Christian doctrine with secular learning (Gracia and Noone 2003). The adoption of learning as a mode of knowledge creation into theological argumentation is a major characteristic of the patristics (as a starting point of medieval philosophy) and of scholasticism which clearly dominated medieval philosophy and which remains influential still. The problems discussed throughout this period are the relation of faith to reason, the existence of God, the purpose of theology, and the problems of knowledge, of universals, and of individuation (Maurer 1982).

\section{Theoretical Background}

The epoch of medieval philosophy can be divided into some main periods: the early period (from the fifth to the twelfth century) followed the patristics which aimed at the preservation and cultivation of ancient philosophy (i.e., Plato and Aristotle). The second period, the so-called golden age of medieval philosophy of the thirteenth and fourteenth century, can be characterized through the culmination of the recovery of ancient philosophy as well as through the seminal work of St. Thomas Aquinas resulting in significant developments in the field of philosophy of religion, logic, and metaphysics. The late period of medieval philosophy (fifteenth century) was characterized by an increasing liberation from the primacy of theology and forming a bridge to the Renaissance (see for an overview: Schoedinger 1996). Representatives of the Renaissance 
showed a tendency to denounce medieval philosophy as the dark and barbaric period between ancient philosophy and its rebirth or renaissance in the fifteenth century. However, in contrast to the relatively short period of the Renaissance, the epoch of medieval philosophy with its duration of nearly 1,000 years stands as the longest period of philosophy in Europe. Moreover, the period of second scholasticism in the sixteenth century as a revival of the scholastic system in opposition to the Protestant Reformation can be argued as an extension of the medieval epoch of philosophy in Europe. Second, scholasticism is strongly associated with St. Ignatius Loyola and the Society of Jesus. The Jesuits' attacks on the Lutheran faith resulted in the development of a kind of Lutheran scholasticism and its counterattacks on the Roman Catholicism. Although second scholasticism declined with the era of the scientific revolution in the seventeenth century, it remained influential in philosophy up to now.

\section{Learning in Medieval Phillosophy}

The adoption of learning as a mode of knowledge creation into theological argumentation is a major characteristics of the patristics which can be traced back to the gnosis of the Apostolic Fathers (of the first and second century) and Justin (second century) who understood learning to be an annexation of single faith truths or the Christian doctrine, in general. In an educational context, learning was occasionally understood as the study and practice of ethical attitudes. Origines (185-254) then developed a foundation of learning by referring to the logos speculation of St. John the Evangelist, whereas St. Augustine of Hippo (354-430) referred primarily to Plato and disapproved of learning as sensual perception. Rather he assumed preexistent associations of intelligible contents in memoria that are refreshed in the activity of learning by means, not unlike Plato's theory of forms. In the following time of the Middle Ages the term "discere" in its general meaning of knowledge acquisition gradually disappeared. Occasionally, we can find the knowledge of Plato's conception of learning, for instance in the writings of St. Albertus Magnus (1193/1206-1280) or in the Quaestiones disputatae from Johannes Picardi of Lichtenberg (1307), but in general there is not any approach of a specific theory of learning at all.

It was St. Thomas Aquinas (1225-1274) who first discussed the problem of knowledge acquisition by referring to Aristotle's concept of learning. In contrast, Master Eckhart (ca. 1260-1327) who was familiar with Plato's epistemology drew learning near to the concept of external experience and considered learning only as an initiating aspect of the process of gnosis.

The synthesis of ancient Greek Philosophy and medieval Christian doctrine resulted in scholasticism which is at the core of medieval philosophy until the emergence of the Renaissance. Scholasticism is derived from the Latin word scholasticus (literally to be understood as "what belongs to the school") and was, first of all, a method of learning taught by the academics (or scholastics) of medieval universities. That is, scholasticism is not a philosophy or a theology in itself, but rather a method for learning with a strong emphasis on dialectical reasoning to resolve contradictions. The most important representatives of scholasticism were Peter Abelard, St. Albertus Magnus, Duns Scotus, William of Ockham, Bonaventura, and St. Thomas Aquinas. The seminal work of St. Thomas Aquinas is generally considered to be the most influential and the epitome of scholasticism (Hyman and Walsh 1973).

The beginning of scholasticism can be shortly characterized by the pursuit of rationality in theology and a strong reference to both ancient Greek philosophy and the adoption of Islamic philosophy. Actually, as European philosophers encountered Islamic philosophy they also encountered new knowledge about mathematics and astronomy. The early thirteenth century saw the culmination of Greek philosophy. For instance, William of Moerbeke's translations and editions of Greek philosophical texts (particularly of Aristotle) in the middle half of the thirteenth century formed a much clearer view on Greek philosophy than the Arabic versions they had been used before. Simultaneously, the first universities were founded in large cities of Europe with the consequence that several clerical orders began to compete in gaining the intellectual control over the universities. In consequence, the emerging scholasticism witnessed the rivalry between the Franciscans and the Dominicans. The Franciscans were founded by St. Francis of Assisi in 1209. Their intellectual leader in the middle of the thirteenth century was Bonaventure, who referred to the theology of St. Augustine and the philosophy of Plato. Other important Franciscan writers were Duns Scotus, Peter Auriol, and William of Ockham. 
The Dominican order was founded by St Dominic in 1215 and placed more emphasis on the use of reason by referring extensively to the new Aristotelian sources derived from the East, and Moorish Spain. The most influential Dominican thinkers in this period were Albertus Magnus and especially St. Thomas Aquinas, whose artful synthesis of Greek rationalism and Christian doctrine resulted in a particular Catholic philosophy. In contrast with the Neoplatonic and Augustinian thinking of the Franciscans, Aquinas was one of the first to refer to new translations of Aristotle's metaphysical and epistemological writing. In consequence, he placed more emphasis on reason and argumentation. Both schools of scholasticism applied what is called the scholastic method.

\section{The Scholastic Method}

The scholastic method consists of both a way of learning and a way of teaching. Scholastic learning or knowledge acquisition occurs in the following way: The scholastics choose a book written by a renowned author as their subject of learning. By reading this book thoroughly and critically, the disciples learn about the theories of the author. In order to give justice to them, other documents and sources related to the subject of learning are analyzed. Anything else written on the subject, be it ancient or contemporary, must be analyzed with regard to disagreements and contentions. The points of disagreement and contention between the multiple sources are captured by sententiae (i.e., individual sentences or statements).

The next step consists in a series of dialectics by which the two sides of an argument are analyzed so that they result in agreement and contradiction. Some arguments can also be completely rejected or new arguments can be proposed. This series of dialectics is done in two ways: philologically and logically. In the philological analysis, words are examined with regard to possible multiple meanings. In addition, it must be taken into consideration that the author may intend a particular word to mean something different. Furthermore, ambiguity can be used to find common ground between two otherwise contradictory statements. The logical analysis relies on the rules of formal logic and aims at a proof that contradictions do not exist. An exposition in accordance with the scholastic method falls in the following components:
1. The questions (quaestiones) as starting point

2. The explanation of the opinions of authorities (sententiae auctorum)

3. The itemization of the pros and cons as well as contradictory statements of authorities

4. The responses to the questions based on logical conclusions and (eventually) the opinions of authorities (solution)

This exposition was especially characteristic for the scholastic method of teaching. Here, two methods can be distinguished. The first teaching method is the lectio wherein learning consists of listening to a teacher who reads a text and expounds on certain words and ideas. Questions are not permitted; students have to listen in silence. The second teaching method is the disputatio undoubtedly the focal point of scholasticism. In the ordinary type of the disputatio a question to be disputed was announced in advance, whereas in the quodlibetal type of the disputatio the disciples propose a question to the teacher without prior announcement. The teacher has to advance a response by citing supportive and authoritative texts in order to prove his position. However, the students then contradict the response, and the quodlibetal continues. Someone has to take notes on the dispute so that the teacher can summarize the arguments and present the final position the following day, riposting all rebuttals.

The scholastic method is characterized through the confrontation of the arguments in form of pro et contra as well as sic et non (yes and no). Regularly, the scholastic method aims at a purification and a resolution of pretended or factual antinomies and antagonisms between authorities by means of a closer determination of the sense and meaning of the statements. Therefore, the scholastic method is also named concordance (concordantia catholica), a method of compromising and reconciliation of pretended contradictions. With regard to their main features, the scholastic methods were not only used widely in the Middle Ages but are also used in modern times.

\section{Important Scientific Research and Open Questions}

The philosophy of the Middle Ages can be characterized in general through the primacy of theology. Philosophy was considered as the maidservant of theology (philosophia ancilla theologiae). Accordingly, the 
theology determined the issues and topics of the philosophical endeavors as well as their scope and range. Truth and knowledge resulted from the dogmas of the Catholic Church. The commitment of philosophy consisted in confirming the "truth" of the dogmas with the help of the scholastic method. The farreaching objective of scholasticism consisted in the consistent integration of the entire traditional and new knowledge in a unique and comprehensive system (summa) which was based on the writings of authorities and the dogmas, respectively.

From here, some critical features of scholasticism resulted, including the undervaluation and disregard of empirical investigation, the abidance in pure conceptual operations, the mere speculation about most general and abstract concepts, the tendency to allembracing but partial systematization, and a substantial intolerance and even militant character.

The major criticism, however, concerns the scholastic method which certainly is not an open-minded and impartial procedure of ascertaining truth, but rather was destined to confirm existing beliefs and opinions. What has not been empirically investigated is to what extent the scholastic method contributed in a positive way to learning. Because some aspects of that method remain in use today, it would be worthwhile to explore impact on learning and the development of higher order reasoning skills in contrast with modern and postmodern approaches.

\section{Cross-References}

- Aquinas, Thomas

- Aristotle

- History of the Sciences of Learning

$\checkmark$ Plato

\section{References}

Ernest, P. (1995). The one and the many. In L. Steffe \& J. Gale (Eds.), Constructivism in education (pp. 459-486). New Jersey: Lawrence Erlbaum Assoc.

Gracia, J. G., \& Noone, T. B. (2003). A companion to philosophy in the middle ages. London: Blackwell.

Hyman, J., \& Walsh, J. J. (1973). Philosophy in the middle ages. Indianapolis: Hackett Publishing.

Maurer, A. A. (1982). Medieval philosophy (2nd ed.). Toronto: Pontifical Institute of Mediaeval Studies.

Schoedinger, A. B. (1996). Readings in medieval philosophy. New York: Oxford University Press.

\section{Equilibration}

Originally, "equilibration" means the act of stabilizing something or making it more stable. In psychology, the term "equilibration" refers to a basic process of development which contains both assimilation and accommodation. According to Piaget, equilibration occurs in three phases: People are in a state of equilibrium, and then they become aware of shortcomings of their thinking and experience a cognitive conflict, which motivates them to adopt a more appropriate idea that eliminates the cognitive conflict and reaches a more stable equilibrium. As in mathematics, the equilibrium can be considered the stationary point of a dynamical system.

\section{Ergometer Cycling}

\section{- Effects of Exercising During Learning}

\section{Erotic/Sexual Learning}

\section{Michael Domjan}

Department of Psychology, The University of Texas at Austin A8000, Austin, TX, USA

\section{Synonyms}

Sexual conditioning

\section{Definition}

Erotic or sexual learning refers to enduring changes in the erotic nature of stimuli or changes in sexual behavior that results from prior experience or learning.

\section{Theoretical Background}

Sexual or reproductive behavior is a fundamental biological function, just as feeding and defensive behavior. Given that a species cannot survive without successful reproduction, one might assume that reproduction would be hard-wired and not dependent on learning. However, sexual behavior can be modified by learning just like feeding and other biologically important 
behaviors. In fact, to date no conditioning effects have been identified that occur in the feeding or defensive behavior system that do not also occur in the sexual behavior system. Under ordinary circumstances, learning improves the efficiency of sexual behavior and increases reproductive success. However, learning can also lead to the development of sexual fetishes that are sometimes detrimental to reproduction.

Most theories of human sexuality have incorporated some form of learning to explain the significant individual differences that are evident in what stimuli people consider to be sexually arousing (Akins 2004). In particular, the development of sexual fetishes or deviant sexual behavior is typically attributed to the learning of an association between an initially nonsexual stimulus or object (e.g., underwear or lace) with sexual satisfaction. However, well-controlled empirical studies of sexual conditioning with human subjects have not appeared until recently.

Human experiments on erotic conditioning typically have used visual conditioned and unconditioned stimuli and have measured learning in terms of changes in genital responses. Hoffmann, Janssen, and Turner (2004), for example tested men and women with a Pavlovian conditioning procedure in which a picture of a gun or the picture of the abdomen of a person of the opposite sex was the conditioned stimulus (CS). The gun was presumed to be unrelated to sex whereas the picture of the abdomen was considered to be a natural or sex-related CS. These stimuli were paired with an unconditioned stimulus (US) that consisted of a $30 \mathrm{~s}$ erotic film clip showing explicit sexual activity. In the control groups, the CSs and the USs were presented unpaired. Learned changes in the erotic nature of the conditioned stimuli were detected by penile tumescence in males and increased vaginal blood flow in women. The naturalistic CS produced a larger conditioning effect than the gun CS in both men and women when the CSs were presented so briefly $(30 \mathrm{~ms})$ that they were not consciously perceptible. When the CSs were presented long enough to be consciously seen $(10 \mathrm{~s})$, the naturalistic CS was again more effective than the gun for men. However, under these conditions the gun CS was more effective than the abdomen CS for women, perhaps because the picture of the gun was more arousing when consciously seen.

In contrast to the relative paucity of well-controlled human experiments on sexual conditioning, numerous studies of sexual conditioning have been conducted in the last few decades with laboratory rats and quail, and a fish species, the blue gourami (Domjan and Akins in press; Pfaus et al. 2001, 2003). These studies have shown that sexual behavior can be modified by a variety of different learning procedures including sexual imprinting, observational conditioning, instrumental conditioning, and classical or Pavlovian conditioning. Sexual imprinting is a learning effect based on rearing history that biases mate choice in adulthood. Male quail, for example, that are raised in cohorts consisting of brown rather than blond quail show a preference in adulthood for mating with brown quail. Observational conditioning occurs if the subjects observe a neutral stimulus paired with a potential sexual partner on a number of occasions. Male quail, for example, that observe an object paired with the appearance of a female quail become more likely to copulate with that object. In sexual instrumental conditioning, an instrumental response, such running down a straight alley, is reinforced by access to a sexual partner in the goal box. As with food reinforcement, getting access to a potential sexual partner increases the rate or speed of the preceding running response. Although imprinting, observational conditioning, and instrumental conditioning are well-established sexual learning effects, most studies of sexual conditioning with nonhuman species have employed classical or Pavlovian conditioning procedures. In these studies, a conditioned stimulus that is initially not effective in eliciting sexually relevant behavior is paired with a sexual unconditioned stimulus (US) on a number of occasions. As a result of these CS-US pairings, the subject's response changes to both the sexual signal and the sexual US.

Visual cues (a colored light, for example) are effective as conditioned stimuli in sexual conditioning with nonhuman species, but numerous other types of stimuli have also been successfully used. If copulation takes places in a distinctive context, rats and quail develop a preference for that context. The presence of sexually conditioned contextual cues has also been found to increase the locomotor activity of male rats in a bi-level chamber, enhance penile reflexes, and decrease the latency to copulate with a female. Olfactory cues are also effective as conditioned stimuli for rats. Males permitted to copulate with a female treated with an artificial odor will subsequently show 
a preference in copulating with a scented rather than an unscented female. In quail, sexual conditioning can develop to distinctive artificial feathers added to a female quail.

The nature of the conditioned stimulus also determines the range of sexual responses that develop as a result of sexual conditioning. Conditioned stimuli that have a distinctive location (e.g., a light or a foam block) come to elicit approach behavior as they become associated with sexual reinforcement. Studies with male quail have shown that if the CS is an object that the male can physically mount and copulate with, conditioned copulatory behavior may also develop. The conditioning of copulatory behavior is facilitated by including some naturalistic features in the conditioned stimulus object.

Studies with nonhuman species have also shown that sexual conditioning can dramatically alter the nature and outcome of courtship and copulation between a male and a female. In blue gourami fish, the presentation of a sexually conditioned stimulus results in decreased aggression, shorter latencies to spawn, increased nest building and clasping behavior, and increased numbers of offspring produced. In quail, sexual conditioning increases cloacal gland contractions and sperm release. Exposure to a sexually conditioned stimulus also decreases the latency to copulate and increases the efficiency of the copulatory behavior. Most importantly, as with the blue gourami, sexual conditioning in quail increases the number of offspring produced. Sexual conditioning also determines the outcome of sexual competition. If two male quail copulate with the same female, paternity of the offspring is determined by which of the males was exposed to a sexually conditioned stimulus before having access to the female.

\section{Important Scientific Research and Open Questions}

In general, evidence of erotic/sexual learning is much more extensive and robust in studies with nonhuman species than in studies with men and women. This is probably due to differences in the theoretical perspectives motivating the research and the learning procedures employed. In research with nonhuman species, sexual conditioning has been examined from the perspective of how learning procedures alter the sexual behavior system broadly conceived, with all of its various appetitive and consummatory components at both the behavioral and neuroendocrine levels of analysis. The research has shown that learned changes occur in all of these components. In contrast, research with human subjects has often employed a more limited perspective that emphasizes the conditioning of reflexive behavior, as evidenced by genital responses. A major challenge for future research is to examine erotic/sexual learning in humans using a broader perspective that includes the entire sexual behavior system rather than just genital responses (Hoffmann in press). It would be interesting to see how sexual learning alters a stimulus in terms of how well the stimulus is liked, how strongly it is preferred, how well it is remembered, how resistant it becomes to subsequent modification, or how much cognitive interference it provides. In parallel with the nonhuman research, one would also want to examine how a sexually conditioned stimulus changes copulatory behavior and the level of satisfaction with copulation.

Studies with human subjects may also benefit from examining a greater range of potential conditioned stimuli, including not just visual cues but also olfactory and tactile cues, as well as three-dimensional objects. Research with both human and avian species also suggests that whether the conditioned stimulus is arbitrary to the sexual behavior system or more naturalistic is also likely to be an important variable.

Another distinctive feature of research with nonhuman species is that copulatory experience was often used to motivate the learning. In contrast, most studies of sexual learning with humans have used visual exposure to film clips of sexual activity as the US. Visual exposure has been found to be less effective as a US than copulation in studies with rats and quail. Human research is beginning to explore the efficacy of genital stimulation as a sexual US. This is an important direction for future research.

Finally, a potentially important variable in studies of sexual learning is the intertrial interval. In studies with rats and quail, the interval between trials has typically been 1 day or more. In contrast, studies with human subjects have presented conditioning trials spaced several minutes apart. Massed trials typically produce less learning than spaced trials. Trial spacing may be an especially important variable in sexual learning because of the long refractory period that typically follows a sexual US or copulation episode. 


\section{Cross-References}

- Classical Conditioning

- Instrumental Conditioning

\section{References}

Akins, C. K. (2004). The role of Pavlovian conditioning in sexual behavior: a comparative analysis of human and nonhuman animals. International Journal of Comparative Psychology, 17, 241-262.

Domjan, M., \& Akins, C. K. (in press). Applications of Pavlovian conditioning to sexual behavior and reproduction. In T. R. Schachtman \& S. Reilly (Eds.), Conditioning and animal learning: Human and non-human applications. New York: Oxford University Press.

Hoffmann, H. (in press). Hot and bothered: Classical conditioning of sexual incentives in humans. In T. R. Schachtman \& S. Reilly (Eds.), Conditioning and animal learning: Human and nonhuman applications. New York: Oxford University Press.

Hoffmann, H., Janssen, E., \& Turner, S. L. (2004). Classical conditioning in the effects of sexual arousal of women and men: Effects of varying awareness and biological relevance of the conditioned stimulus. Archives of Sexual Behavior, 33(1), 43-53.

Pfaus, J. G., Kippin, T. E., \& Centeno, S. (2001). Conditioning and sexual behavior: A review. Hormones and Behavior, 40, 291-321.

Pfaus, J. G., Kippin, T. E., \& Coria-Avila, G. (2003). What can animal models tell us about human sexual response? Annual Review of Sex Research, 14, 1-63.

\section{Error of Substitution}

Substitution Errors in Learning

\section{Errorless Learning}

New Learning in Dementia

\section{Essentialism}

The belief that various characteristics or traits are innate to particular classes of objects or subjects; the philosophical belief that all entities of a certain kind must possess certain characteristics independent of context. One application of essentialist thinking is that women are born with superior language skills, while men are born with superior math skills. Such reasoning does not occlude the possibility of other traits or externalities (such as how a child is raised), but those traits are not seen as being determinate of the essential characteristic, or essence, of the group.

\section{e-Tandem Learning}

Tandem Learning

\section{Ethics Learning}

- Moral Learning

\section{Ethnomusicology in Education}

- Multicultural Issues in Music Instruction and Learning

\section{Evaluation}

Assessment in Learning

\section{Evaluation of Learning}

Diagnosis of Learning

\section{Evaluation of Management, Leadership, and Organizational Development}

JOHN Burgoyne

Department of Management Learning and Leadership, Lancaster University Management School, Lancaster University, Lancaster, Lancashire, UK

\section{Definition}

Evaluation of management leadership and organization development (and indeed any other purposeful 
human activity) is about asking whether it is working and whether it could work better, both in terms of the purposes that various stakeholders have for it, and others that can be envisaged. A definition, drawing on Pawson (Pawson and Tilley 1997) and Patton (Patton 1996) might be: the assessment of the impact of an initiative, in terms of both intended and unintended consequences, and the process by which this is achieved, to inform decisions both about its value for money and ways of improving it.

\section{Theoretical Background}

It is useful to distinguish between small "e," natural, informal evaluation that goes on in corridor conversations and big "E" evaluation, deliberate and systematic efforts to evaluate in an evidence-based way.

It is easy to make a logical and rational case for Evaluation, along the lines that there is no point in shooting at a target unless you look to see if you have hit it with a view to improving your aim.

However, this is rarely the case in practice, this kind of development often being seen as an "act of faith," and one that is difficult to evaluate anyway.

Evaluation tends to be more common in the public sector rather than the private sector where there are more requirements for accountability and reporting.

In harsh economic situations the logical case for Evaluation is stronger, since it should ensure value for money, and this is taken slightly more seriously than a number of years ago. However, it is equally likely that Evaluation will be cut, and quite possibly the activity being evaluated too, since it is of uncertain value and, at best, long-term benefit.

The author succeed, briefly, in persuading a public sector leadership center in the UK to adopt a policy of investing $10 \%$ of any development budget in Evaluation. However, despite this, the center in question switched much of its efforts from leadership development to shorter-term "lean" initiatives, with methodologies like Six Sigma, since they are shorter term, more local, and easier to Evaluate.

By far the best known model for evaluation is Kirkpatrick's (Kirkpatrick 1996). This envisages a chain of consequences from a development activity with four links: the experience itself for the target group (called the reaction level, usually manifest in the so-called happy sheets), then learning (the learning level), then the application of that learning (the behavior change level), and finally the benefit level (which is usually to the organizational entity on behalf of which the initiative being evaluated is carried out, but can be to other interests, for example, the individual or society in general).

The Kirkpatrick model occupies something of a dominant position, often to the exclusion of anything else. Even an attempt to get "Beyond Kirkpatrick" still has it as the dominant underlying framework (Tamkin et al. 2002).

There is a useful development of the model formulated by the UK National College for School Leadership (now just the National College), which is particularly appropriate to a start up initiative with aspirations beyond the organizational. The stages are something like: program awareness, program appreciation, program use, program enjoyment (the reaction level), local application, broader application, and system improvement (where, for example, the schools in a region work better together). The early stages of the model have something of a marketing flavor, which is arguably appropriate to a new initiative, especially where its use is somewhat voluntaristic.

The Kirkpatrick model can be used in a "hard science" way, with objective before and after measurements and an experimental design with a control group that does not undergo the intervention being evaluated, or, as is more normal, in a soft, qualitative way where subjective judgments are collected and combined, though hopefully with some attempt to objectify the observations. It is usually the case that attributing causality is the more challenging problem than the measurement itself. Jack Phillips, the champion of Return on Investment (ROI) evaluation, goes to objective measures but has to make do with subjective attributions of causality by the people closest to the action (Phillips 1994).

Some other useful models are:

1. Easterby-Smith, who argues that it is useful to be clear about the aims of and evaluation project, and offers a $2 \mathrm{X} 2$ of formative (making it better) and summative (proving it works), and process (the means) and ends (the outcome). This generates proving, improving, monitoring, and learning (Easterby-Smith 1994).

2. Patton, who argues that any evaluation project should be client focused and start by asking who the client is, what decision they want to make better 
in the light of the evaluation, and then design data collection, analysis, and reporting to make evidence-based recommendations that are as good as possible within the constraints of resources and time (Patton 1997).

3. Burgoyne, who suggests a multi-stakeholder approach to evaluation, which suggests that multiple stakeholders, with aligning and conflicting interests, need to be taken into account (Burgoyne 1994).

These models can be combined in a number of pragmatic ways, for example Kirkpatrick, with a main stakeholder agenda, but also subsidiary stakeholders, and client themselves as one of the stakeholders.

\section{Important Scientific Research and Open Questions}

Evaluation shares all the usual methodological problems with organizational research in general. There are two main issues: measuring change and attributing causality. Both are difficult but the latter more so. Critical Realism (which takes the world to be an open system with emergent properties) seems to be the most promising methodological approach, in contrast to positivism (which sees the world as a deterministic machine) and extreme social constuctionism (which takes the world to be a shifting sea of cultural meaning). This approach has been best developed and exemplified by Pawson and Tilley (1997).

The second and last issue is that evaluation is usually instrumental; it takes some change objective as given and assesses the whether and how of progress toward it. However, evaluation research rarely engages with the evaluation of the rightness and appropriateness of the overall objective. The possibility of this is philosophically debated, but it is possible to argue that a good objective is a move toward long-term sustainability for the whole cosmic system including, but not exclusively, humanity. Most evaluation is, in Argyris's terms (Argyris 1977), single- rather than double-loop learning, that is, questioning whether an outcome is achieved rather than whether the outcome is worthwhile.

\section{Cross-References}

- Affordances

- Cybernetic Principles of Learning

- Design Experiments
- Double-Loop Learning

- Evidence-Based Learning

- Feedback and Learning

- Learning Objectives

- Measurement of Learning Processes and Outcomes

- Self-managed Learning

\section{References}

Argyris, C. (1977). Double loop learning in organizations. Harvard Business Review, 55(5), 115-125.

Burgoyne, J. G. (1994). Stakeholder analysis. In C. Cassell \& G. Symon (Eds.), Qualitative methods in organizational and occupational psychology. London: Sage.

Easterby-Smith, M. P. V. (1994). Evaluation of management education, training and development. Aldershot: Gower.

Kirkpatrick, D. L. (1996). Great ideas revisited: Revisiting Kirkpatrick's four-level model. Training and Development, 50(1), 54-57.

Patton, M. Q. (1996). Utilization focused evaluation. London: Sage.

Patton, M. Q. (1997). Utilization-focused evaluation - The new century text. London: Sage.

Pawson, R., \& Tilley, N. (1997). Realistic evaluation. London: Sage.

Phillips, J. (1994). ROI: The search for best practice. American Society for Training and Development, 50(5), 42-48.

Tamkin, P., Yarnall, J., \& Kerrin, M. (2002). Kirkpatrick and beyond: A review of training evaluation. Institute of Employment Studies: Brighton.

\section{Evaluation of Student Growth in Learning}

- Evaluation of Student Progress in Learning

\section{Evaluation of Student Progress in Learning}

HoI K. Suen ${ }^{1}$, Hongli LI ${ }^{2}$

${ }^{1}$ The Pennsylvania State University, University Park, PA, USA

${ }^{2}$ Department of Educational Policy Studies, Georgia

State University, Atlanta, GA, USA

\section{Synonyms}

Assessing student progress in learning; Evaluation of student growth in learning 


\section{Definition}

Evaluation of student progress in learning focuses on the questions of whether students have learned and how much they have learned. This is in contrast to evaluating student proficiency at a single time point. To evaluate student progress in learning, we need to conduct assessment at different time points in order to observe changes over time. The information obtained may be expressed informally in the form of verbal feedback, in the form of a numerical gain score, or in the form of growth curves. These pieces of information are used either for accountability purposes or for instructional feedback.

\section{Theoretical Background}

The progress made by a student may be expressed in the form of verbal or written comments or in numerical scores or both. In general, for the purpose of instructional feedback, verbal or written comments are more appropriate as they tend to be more informative and richer in detail and scope. For the purpose of accountability, however, reliability and validity of information and interpretation are more important. It is generally easier to ascertain reliable and valid numerical scores than it is to ascertain reliable and valid verbal descriptions.

At its basic level, teachers can verbally provide informal descriptive feedback regarding a student's learning process during a certain time period. The major advantage of such informal verbal feedback is its timeliness and such feedback can be quite rich in details. The verbal feedback can be readily understood, and necessary actions can be discussed and taken accordingly. Alternatively, such feedback can be provided more formally in writing. A rubric may be provided to help interpret the written feedback. Regular feedback, oral or written, formal or informal, is in itself very helpful to the learning process.

For the purpose of accountability or for comparisons, verbal or written feedback of progress is inadequate, because such feedback tends to be insufficiently structured and the information provided is often imprecise. Numerical scores are used instead. Common numerical expressions of progress in learning include gain scores and growth curves. Gain scores are obtained by calculating the change of test scores from a pretest to posttest. If there is a gain from a pretest to posttest, such gain is interpreted as an indication that learning progress has occurred. The magnitude of the gain score is then used to calibrate the amount of progress.

Though conceptually and computationally simple, gain scores can be quite difficult to interpret due to a number of factors. A common concern is the potential effect of regression to the mean. Due to the unreliability of test scores and other random situational fluctuations of scores, a posttest score is expected to be at least slightly, and sometimes considerably, different from the pretest score; even when no actual learning progress has occurred. If the pretest score is very low, such random fluctuation alone will tend to lead to a higher posttest score. Conversely, if the pretest score is very high, the posttest score will tend to be lower. Thus, it is difficult to discern how much of an observed gain in test scores for a student is due to this regression artifact and how much is due to genuine progress in learning.

Another commonly known problem with gain scores is that, if the same test form is used for both the pretest and the posttest, at least part of the gain in scores can be attributed to practice effect of having taken the pretest. Even though learning did take place (i.e., learning from the pretest), this progress is due to having been given a pretest, not due to instruction. As such, when the same test is used for pretesting and posttesting, using gain scores as indicators of progress for accountability purposes can be misleading.

An apparent solution is to use different forms of a test as the pretest and the posttest. This approach is by necessity the general practice when progress is assessed over a number of years or grade levels. This is because, as a child ages and moves into higher grades, the early low-level pretest is no longer appropriate and a more advanced or difficult test is needed to measure that child's ability. When the pretest and the posttest are different forms of a test, any apparent score gain can be attributed to possible difference in difficulty between the two forms. In this case, statistical adjustments are needed to remove score differences due to form differences so that scores are comparable before gain scores can be calculated and evaluated. The adjustments are generally accomplished via one of a variety of psychometric techniques known as equating. Through equating, performances on different forms of the test are scaled on a common metric after differences in difficulty and other characteristics have been accounted for. To calibrate student progress from 1 year to another 
year and/or between grade levels, a class of equating technique called vertical equating is particularly appropriate (see Kolen and Brennan 2004 for details).

Gain scores gauge the total change of scores between two specific points in time. It is inherently static and does not provide sufficient information to discern trend or pattern of change. In contrast, growth curve analyses show not only whether there has been a gain in learning, but also the pattern through which progress has occurred over a time period. Values of the measured performance can be plotted on a graph as a function of time. The scores can be observed as either increasing or decreasing over time. The overall pattern of change may be linear or nonlinear.

In its simplest form, growth curves are frequently used in single-case research and applied behavior analyses, mostly within the field of special education, to evaluate the efficacy of a particular intervention or teaching strategy. Even though the approach is similar to that of time-series analysis or interrupted time-series analysis, in that many observations of a student's performance are taken over time and the scores are plotted graphically, advanced statistical techniques are generally not used in these single-case or applied behavior analyses. Instead, the growth curve is inspected visually to detect trends and patterns.

In addition to visually inspecting growth curves, we can statistically describe these curves as linear trends or nonlinear trends. The latter may include such variations as power curves, polynomial curves, cyclical trends, or a number of other patterns. The most appropriate trend is typically identified by the degree of data fit. Just like gain scores, the observed trend in a growth curve may be caused by factors other than learning. For example, the observed pattern of change may be no more than a continuation of preexisting natural maturation process; or an observed upward trend may be no more than a segment of a larger up and down cyclical trend. To determine how much of the change is attributable to learning or intervention, it is necessary to first remove these potential natural trends from the data prior to evaluating growth. Methods such as BoxJenkins ARIMA techniques are appropriate for this purpose; but are in reality rarely used in education due to the fact that these techniques require dozens to hundreds of data points over time - a requirement that is typically impractical for educational evaluation.
When we wish to assess the overall growth of a group, a class or a school of students, in addition to all the factors discussed above, we also need to attend to potential errors due to sampling of students. Typically, for these group analyses, statistical modeling methods that can discern the underlying latent growth curves are used. Some of the commonly used methods include hierarchical linear modeling and structural equation modeling. These are large-sample statistical techniques and are, thus, useful for large-scale assessment program (see Kline 2005 for details).

Whether and how growth has occurred is most usually a relative judgment by comparing scores at different points in time within the growth curve. However, it is also possible to evaluate growth against some absolute standard. For example, in the implementation of the No Child Left Behind (NCLB) legislation in the USA, the US Department of Education mandated that growth models must adhere to the major requirement that each student will be proficient in reading and math by the year 2014. Thus, to meet this NCLB mandate, growth models must focus on "growth toward proficiency" (Dunn and Allen 2009).

\section{Important Scientific Research and Open Questions}

Information about student progress in learning can be used as instructional feedback or for accountability. A currently popular model that utilizes such information as feedback for students with disabilities is the Response-to-Instruction (RTI) approach. It involves screening at-risk students, monitoring student progress in response to instruction, and determining the appropriate course of action based on progress. A somewhat related approach that uses progress information as instructional feedback is Dynamic Assessment (DA). A focus of DA is to offer assistance to students as part of the assessment process, so that we not only assess a student's current ability, but also the student's learning process and what pedagogy or intervention best facilitates the student's learning. Dynamic assessment is thus a pretest-teach-posttest loop, and the distinction between assessment and instruction is intentionally blurred. Curriculum-based assessment is yet another model through which progress assessment information is used as feedback to guide instruction. Perhaps due to the need for flexibility in instruction, 
these approaches have been used primarily in special education in which individualized educational plans are implemented.

For group instruction, student progress in learning is monitored at the class or school level. The data have been primarily used for accountability purposes. These include the determination of whether a particular educational program has produced the desirable effect, the evaluation of the quality of education at the class or school level; and to evaluate whether sufficient progress has been made toward a particular standard. The assessment required by the NCLB is a typical case of using the evaluation of student progress for accountability with standard-based assessment design. Adequate Yearly Progress (AYP) is one of the cornerstones of the NCLB, which refers to an annual measure of student participation and achievement of statewide assessments and other academic indicators. Most states have designed standardized tests that students have to take to measure whether schools have made their individual AYP. Through the metric of AYP, states, school districts, and schools are held accountable for student progress in learning.

There has been a fundamental tension between using student progress evaluation for instructional feedback and for accountability. To be useful for feedback, emphasis needs to be placed on the richness of information. As such, large amounts of fine-grained student progress data need to be collected on many aspects of learning. Some of these data would necessarily be unstandardized and may have low reliability. To be useful for accountability, student progress data need to be accurate, standardized, and comparable from student to student. Often, the aspects of learning being evaluated would be limited. Therefore, a standardized assessment designed for accountability is often not very useful for instructional feedback. The former tends to use gains in large-scale standardized test scores to express progress in learning; while the latter tends to rely on informal and often verbal feedback. However, recent advances in a particular psychometric analytic approach known as Cognitive diagnostic modeling (CDM) has made it possible, at least theoretically, to obtain both reliable standardized scores to evaluate progress for accountability and finegrained detailed multidimensional data for instructional feedback simultaneously.

\section{Cross-References}

- Assessment in Learning

- Effects of Testing on Learning

- Test-Enhanced Learning

\section{References}

Dunn, J., \& Allen, J. (2009). Holding schools accountable for nonproficient students: Coordinating measurement and accountability. Educational Measurement: Issues and Practice, 28(4), 27-41.

Kline, R. B. (2005). Principles and practice of structural equation modeling. New York: The Guilford Press.

Kolen, M. J., \& Brennan, R. L. (2004). Test equating, scaling, and linking: Methods and practices (2nd ed.). New York: Springer.

\section{Evaluation of Testimony}

- Children's Critical Assessment of the Reliability of Others

\section{Evaluative Conditioning}

Jan De Houwer

Ghent University, Ghent, Belgium

\section{Synonyms}

Affective conditioning; Affective learning; Associative transfer of valence; Evaluative learning

\section{Definition}

Evaluative conditioning is most often defined as an effect rather than as a mental or neural process. As an effect, evaluative conditioning is a change in the valence of a stimulus that is due to the prior pairings of that stimulus with another stimulus (De Houwer 2007). The first stimulus is most often referred to as the conditioned stimulus (CS). The second stimulus is called the unconditioned stimulus (US). Evaluative conditioning is a subset of Pavlovian conditioning. Whereas Pavlovian conditioning can refer to any type of change that results from the pairing of stimuli, evaluative conditioning by definition involves a change in liking. Evaluative conditioning differs 
from other learned changes in liking (e.g., the mere exposure effect) in that the change in liking is due to the pairing of stimuli rather than other regularities in the environment (e.g., the repeated occurrence of a stimulus).

\section{Theoretical Background}

The term Evaluative Conditioning was first used by Levey and Martin (1975), but earlier demonstrations of the effect have been reported (see De Houwer et al. [2001], and Hofmann et al. [2010], for reviews of the literature). As the word "conditioning" suggests, evaluative conditioning research was initially closely tied to the learning literature, more specifically, the literature on Pavlovian conditioning. Because preferences are assumed to be an important determinant of many aspects of behavior and cognition, interest in how stimulus pairings can lead to changes in preferences soon spread to consumer psychology, clinical psychology, emotion research, and social psychology. Research on evaluative conditioning can help us understand how preferences are acquired during the lifetime of organisms.

\section{Important Scientific Research and Open Questions}

Given its origins in learning psychology, research on evaluative conditioning primarily dealt with the question of whether and how evaluative conditioning differs from other forms of Pavlovian conditioning (see De Houwer et al. [2001], and Hofmann et al. [2010], for reviews). Initial research suggested that there are differences. First, whereas Pavlovian conditioning relies heavily on awareness of the CS-US contingencies, some findings suggest that evaluative conditioning can occur without contingency awareness. Second, conditioned changes in liking seem to persist even when the CSs are presented on their own after conditioning. Hence, evaluative conditioning appears to be less sensitive to extinction than other forms of Pavlovian conditioning. Because of these differences, some authors suggested that evaluative conditioning depends on more basic learning processes than other forms of Pavlovian conditioning. Most prominently, evaluative conditioning has been attributed to a Hebbian-like learning mechanism in which the formation of associations in memory is driven primarily by co-occurrences.
More recent findings, however, question the idea that evaluative conditioning is a unique form of conditioning that relies on a unique type of association formation process. Many studies have been reported which show that evaluative conditioning does crucially depend on contingency awareness. A recent meta-analysis (Hofmann et al. 2010) confirms that contingency awareness is by far the most important moderator of the size of Evaluative Conditioning. The meta-analysis also revealed a significant extinction effect, be it a relatively small one. Other recent studies showed that evaluative conditioning is similar to other types of Pavlovian conditioning also in that it depends on attention and goals and can result from instructions rather than actual stimulus pairings (e.g., Pleyers et al. 2009). These findings suggest that evaluative conditioning depends on the same mental processes than other types of Pavlovian conditioning. Some have argued that both types of learning are mediated by formation of propositions, but because of the many conflicting findings, strong differences in opinion exist about the conditions that moderate evaluative conditioning and the mental processes that mediate it (see Hofmann et al. 2010). It is possible that the divergent results are due to the fact that evaluative conditioning can be produced by different kinds of mental processes (De Houwer 2007). Sometimes, evaluative conditioning might be due to simple associative mechanisms, in which case it would be independent of contingency awareness and attention. Other instances of evaluative conditioning might be due to propositional processes and thus be dependent on contingency awareness and attention. Although this dual-process hypothesis seems plausible, it raises important questions about how the processes interact and what variables determine when a particular process will drive Evaluative Conditioning.

Despite the uncertainty about the conditions under which evaluative conditioning occurs and the mental processes that mediate Evaluative Conditioning, there is general agreement about the fact that evaluative conditioning is a genuine phenomenon that contributes to many different aspects of behavior (see Walther et al. 2005, for a review). In consumer psychology, evaluative conditioning is seen as an important source of attitudes towards products and brands. In clinical psychology, it has been proposed that evaluative conditioning contributes to (the return of) fear. 
In social psychology, evaluative conditioning has been linked with a variety of topics such as attitude formation, ingroup favoritism, prejudice, and stigmatization. Although there are boundary conditions to when evaluative conditioning occurs, the available literature shows that evaluative conditioning is a general phenomenon that has been observed with many different types of stimuli. In all likelihood, Evaluative Conditioning will thus remain an important topic in many areas of psychology.

\section{Cross-References}

- Associative Learning

- Attitude Change (Through Learning)

- Attitudes - Formation and Change

Conditioning

- Dual Process Learning Model

- Pavlovian Conditioning

- Taste Aversion Learning

\section{References}

De Houwer, J. (2007). A conceptual and theoretical analysis of evaluative conditioning. The Spanish Journal of Psychology, 10, 230-241.

De Houwer, J., Thomas, S., \& Baeyens, F. (2001). Associative learning of likes and dislikes: A review of 25 years of research on human evaluative conditioning. Psychological Bulletin, 127, 853-869.

Hofmann, W., De Houwer, J., Perugini, M., Baeyens, F., \& Crombez, G. (2010). Evaluative conditioning in humans: A meta-analysis. Psychological Bulletin, 136, 390-421.

Levey, A. B., \& Martin, I. (1975). Classical conditioning of human evaluative responses. Behaviour Research and Therapy, 4, 205207.

Pleyers, G., Corneille, O., Yzerbyt, V., \& Luminet, O. (2009). Evaluative conditioning incurs attentional costs. Journal of Experimental Psychology: Animal Behavior Processes, 35, 279-285.

Walther, E., Nagengast, B., \& Traselli, C. (2005). Evaluative conditioning in social psychology: Facts and speculations. Cognition and Emotion, 19, 175-196.

\section{Evaluative Learning}

Evaluative Conditioning

\section{Evaluative Response Signal}

- Feedback in Instructional Contexts

\section{Evaluative Response Strategies, Planned and Coordinated Sequence of Post-Response Information}

Feedback Strategies

\section{Event Memory}

- Episodic-Like Memory in Food-Caching Birds

- Memory for "What," "Where," and "When" Information in Animals

\section{Everyday Consciousness}

- Learning Defense

\section{Everyday Learning}

- Comprehensive Learning

- Learning in Practice and by Experience

\section{Everyday Learning: Instruction and Technology Designs}

\section{Brad Mehlenbacher}

Department of Leadership, Policy and Adult and Higher Education, North Carolina State University, Raleigh, NC, USA

\section{Synonyms}

\section{Evaluative Priming}

- Affective Priming and Learning 


\section{Definition}

Instruction and technology designs for everyday learning is a multidisciplinary and emerging area of research that places design at the heart of the complex relationship between instruction, learning, and technology. Instruction and learning with technology involves any technology (e.g., telegraph, telephone, typewriter, digital information and communication technology, etc.) that disturbs normative space and time, either in terms of our perception of what is real and what is artificial or of the passage of time. Technology, digital or otherwise, is not merely utilitarian or instrumentalist in its influence on the way we instruct and learn; nor does technology degrade or enhance instruction and learning in uniform ways. Technology use in instruction and learning is a complex domain for study, primarily because it is exceedingly difficult to define the precise natures of technology, instruction, and learning.

\section{Theoretical Background}

Research related to instruction and learning with technology is found in numerous disciplines and subdisciplines, such as distance learning and e-learning; educational, instructional, and communication technology; teaching and learning sciences; communication and information design; human-computer interaction and ergonomics; in peer-reviewed journals related to training, adult education, and the workplace; education in the humanities and social sciences; education in science, technology, engineering, and mathematics; and others. Well-defined disciplinary areas of inquiry require minimal framing for their arguments. The disparate and exponentially growing research related to instruction and learning with technology, however, demands extensive background study and a rearticulation of the range of methods available to researchers interested in the topic (Mehlenbacher 2010). Fundamental terminologies must be defined. What do we mean when we talk about learning? What do we know about effective instruction? What is the relationship between the way humans learn and the methods that we use to instruct them? What is it we are referring to when we speak of technology?

The rationale for developing an understanding of the relationship between instruction, learning, and technology is that their interaction artificializes our definitions of learning and the learner, instruction and its relationship to the classroom, resources and artifacts for instruction, and the lifelong learning that pervades our professional and personal learning worlds. The technologies that distribute our classrooms across time and space necessitate a rearticulation of what we value as natural about our non-distributed "traditional" classrooms.

Learning researchers (e.g., Bransford et al. 2000) have elaborated for the last several decades on the following critical information-human interactions: (1) information and comprehension, (2) representation and integration with existing and available knowledge structures, (3) retrieval and development of new connections between the new information and the existing state of understanding, and (4) construction and elaboration toward a richer understanding of the subject matter, leading to expert understanding. Numerous researchers have broadened this cognitive perspective to include perceptual and discourse processes (Evans 2004) and social views that include activities, motivation and engagement, social interaction, and complex learning environments (Grabinger 2004). A rich perspective toward learning, therefore, is one that draws on various quantitative and qualitative traditions, constructing alternative conceptions of the learning process depending on our methodological viewpoint (see Table 1).

Instruction and learning with technology requires both a usability perspective and a rhetorical perspective toward our objects of inquiry. A usability perspective toward instruction and learning with technology emphasizes how technology supports or impedes learner tasks with instructional materials and artifacts. A rhetorical perspective maintains that the relationship between a rhetor and audience (i.e., the instructor and the learner) ought to be the foundation on which all other instructional issues rest. Influenced by Buchanan (1992), a rhetorical design perspective toward instruction and learning with technology requires the application of symbolic and visual communication, of material objects, of activities and organized services, and of complex systems or environments for living, working, playing, and learning (pp. 9-10). Design is what we do when we conceive and craft technical solutions for instructional situations using a combination of general (i.e., theoretical) knowledge and situational (i.e., practical) knowledge. Design is what instructors and instructional designers do when they explore and manipulate the capabilities of virtual learning 
Everyday Learning: Instruction and Technology Designs. Table 1 Alternative views of the same learning activities or processes (Adapted from Mehlenbacher 2010)

\begin{tabular}{|l|l|l|}
\hline Approach & Emphasis & Specific methods \\
\hline Biophysical & Physical, motor, neurological & $\begin{array}{l}\text { Electronencephalography (EEG), ERPs, fMRI, MEG, NIRS, PET, } \\
\text { TCDS }\end{array}$ \\
\hline Behavioral & Behavior, perception, tasks & $\begin{array}{l}\text { Direct observation, trial and error, punishment/reward, } \\
\text { time-stamping }\end{array}$ \\
\hline Cognitive & $\begin{array}{l}\text { Mind as computer (attention, retrieval, } \\
\text { mental models, cognitive overload) }\end{array}$ & $\begin{array}{l}\text { Talk-aloud protocols, computer modeling, quantitative } \\
\text { experimental designs }\end{array}$ \\
\hline Organizational & Tasks in context & $\begin{array}{l}\text { Activity theory, genre analysis, case study approach, social } \\
\text { network analysis }\end{array}$ \\
\hline Cultural & Human interaction & $\begin{array}{l}\text { Situativity theory, discourse analysis, critical realism, } \\
\text { political theory }\end{array}$ \\
\hline Historical & History, events, chronology & $\begin{array}{l}\text { Anthropological approaches to situativity and community } \\
\text { formation, structural and poststructural analysis, } \\
\text { phenomenology }\end{array}$ \\
\hline
\end{tabular}

environments. Indeed, design is what we expect successful learners to do when we attempt to teach them to interpret, analyze, present, and creatively apply new knowledge to their prior understandings and to novel problem situations.

At the heart of any instructional situation is the learner and, so, synthesizing historical and emerging developments on learning is critical to our understanding of how to design effective instructional situations. Beginning with a theory of learning that builds on information processing, our definition of learning includes environmental cues through sensoryperceptual processes through cognitive processing to actions within the learning environment. A sociocognitive perspective toward instruction and learning with technology sidesteps traditional dichotomies between cognitive and social theories of learning by factoring sensory-perceptual processes into the equation.

Designing instruction, then, is the act of combining the elements of content and display to effectively present instructional content in a way that promotes learning through organized instructional resources and user interfaces that are not confusing, dissatisfying, or cognitively taxing. More than that, designing effective instruction involves balancing five broadly conceived dimensions of everyday instructional situations (see Table 2).
Ultimately, the benefit of taking a rhetorical design perspective toward instruction and learning with technology is that it helps us achieve three goals: encouraging a multidisciplinary, multi-methodological approach to studying, designing, and evaluating instructional situations and learning events, understanding theory and practice regarding instruction and learning with technology, and building usable learning environments that facilitate the various dimensions of everyday instructional situations.

\section{Important Scientific Research and Open Questions}

Research on instruction and learning with technology is such an emerging area of inquiry that it invites more questions than immediate answers. Certainly, the intersection of technology, cognition, instruction, and learning should be an essential object of inquiry for all educational researchers and providers. Moreover, accelerated developments in the area currently traverse traditional disciplinary borders and demand that research be drawn from multiple disciplinary perspectives.

For the last 30 years, the majority of the research on instruction and technology designs for everyday learning has focused on media comparison studies, where technology is treated as an experimental variable that either does or does not influence learning (frequently 
Everyday Learning: Instruction and Technology Designs. Table 2 Five dimensions of everyday instructional situations (Adapted from Mehlenbacher 2010)

\begin{tabular}{|l|l|}
\hline Dimensions & Relevant activities or attributes \\
\hline $\begin{array}{l}\text { Learner background and } \\
\text { knowledge }\end{array}$ & $\begin{array}{l}\text { The biological attributes (age, gender, race/ethnicity), abilities (cognitive, physical), } \\
\text { personal identity (learning style, attitude, motivation, self-monitoring), literacies } \\
\text { (computer, domain, textual, visual), and sociocultural context (family, economic, } \\
\text { geographic, organizational) of learners in formal and informal instructional situations }\end{array}$ \\
\hline Learner tasks and activities & $\begin{array}{l}\text { The nature of tasks (e.g., requirements, procedures, importance, frequency, time, } \\
\text { complexity), user behaviors and expectations, and human problem-solving activities with } \\
\text { learning materials, exercises, goals, processes (e.g., reading to learn, reading to learn to do, } \\
\text { reading to analyze, reading to compare, confirm, and correct) }\end{array}$ \\
\hline Social dynamics & $\begin{array}{l}\text { Socio-emotional-affective-cognitive interactions between learners and instructors, } \\
\text { learners and learners, learners and content, learners and interfaces, instructors and } \\
\text { content, instructors and interfaces, instructors and other instructors, content and content, } \\
\text { and institutional support structures that occur in instructional situations }\end{array}$ \\
\hline Instructor activities & $\begin{array}{l}\text { Audience adaptation, communication of content, objectives, prior knowledge, information } \\
\text { exchange, topic elaboration, topic pacing and flow, sequencing, methods of evaluation, } \\
\text { and immediacy of feedback, in addition to understandings of subject matter, theories of } \\
\text { knowledge, pedagogy, and reflective abstraction }\end{array}$ \\
\hline $\begin{array}{l}\text { Learning environment and } \\
\text { artifacts }\end{array}$ & $\begin{array}{l}\text { Ergonomic design for usability, comfort, functionality, and aesthetics that supports design } \\
\text { for two worlds (face to face and virtual) and for three worlds (face to face, virtual, and } \\
\text { conceptual) }\end{array}$ \\
\hline
\end{tabular}

defined by course grades or test scores). Not surprisingly, these studies have resulted in no significant differences between instruction with technology and instruction without. However, these studies could benefit significantly from the incorporation of other significant factors in the learning process. For example, how do learner backgrounds and knowledge influence learning processes and outcomes, how do technologies enhance and impede learner tasks and activities, how do instructional technologies influence social dynamics, and how do the learning spaces that we inhabit and the artifacts that we create support or undermine our everyday learning situations?

Despite the explosive growth of peer-reviewed journals devoted to distance education and instruction and learning with technology, research has tended to emphasize recent technological developments and particular "tools" and their influence on instruction and learning (e.g., social networking and gaming). But many of the technical solutions and research-based design principles governing current instructional design would have applied to the hypertext systems of the late-1980s as much as they may have applied to the designs of historical paper-based correspondence courses. Researchers could benefit from drawing on research from alternative disciplines (e.g., leisure studies, training, rhetorical theory, and design studies) and from beginning to amass historical accounts of technology use that might inform our current design situations.

As well, taking a transformational rather than instrumental perspective toward the role of technology use in instruction and learning necessitates that we view all instruction as technologically mediated. Both traditional lecture-style presentations and virtual reality simulations are mediated learning experiences. It is problematic to reduce instruction to content, modules, objects, or units. Instruction cannot occur without information, and information requires communication. To be a communication act, something must be articulated, inquiry must occur, interpretations must be organized as arguments. These acts require community and context. Because all instructional situations are communication acts, what is important and what requires additional research is how the act of learning is transformed, how we assess whether learning has occurred, and how we learn to instruct more effectively.

In addition to the bulk of media comparison studies characteristic of research on technology use in instruction and learning, considerable research has 
documented how learner background and knowledge contribute to successful learning. However, these studies have focused in particular on gender differences and constructs such as learning styles, leaving a great many learner attributes unexamined. For example, more research is needed on how biological variables such as age and ethnicity and physical attributes (e.g., haptic or ambulatory) influence learning. Research on how different levels of experience (with technology, domain knowledge, and with textual and visual information processing) can help us better understand learner literacies. And sociocultural variables such as socioeconomic and geographical factors and their influence on learning in given instructional situations require considerable elaboration.

Finally, instruction and learning with technology has tended to emphasize either learning inside the box (computer-based instruction) or face-to-face instruction that is augmented by technology (blended designs for two worlds). A third, emerging area of research is described by Bereiter (2002) when he introduces "conceptual artifacts." Conceptual artifacts are immaterial objects constructed of discourse that, according to Bereiter (2002), "serve purposes such as explaining and predicting" and that focus on "discussible ideas, ranging from theories, designs, and plans down to concepts, like unemployment and gravity" (p. 64). We can begin to imagine conceptual (e.g., virtual) artifacts as ideas that can be realized or simulated in virtual environments. For example, designers have built simulations of atoms, coral reef patterns, and star trajectories; conceptual artifacts could also include collaborative documents that simulate team editing or social networking patterns that evolve as new variables are added to the interaction plan. Research on the design and evaluation of virtual artifacts is in its early stages but, as the quality of immersive graphical environments continues to improve, promises to creatively contribute to current understandings of instruction and learning with technology.

\section{Cross-References}

- Design of Learning Environments

- Ecology of Learning

- e-Learning and Digital Learning

- Learning by Design

- Social Cognitive Learning

- Virtual Learning Environments

\section{References}

Bereiter, C. (2002). Education and mind in the knowledge age. Mahwah, NJ: Lawrence Erlbaum.

Bransford, J., Brown, A. L., Cocking, R. R., \& National Research Council. (2000). How people learn: Brain, mind, experience, and school. Washington, DC: National Academy Press.

Buchanan, R. (1992). Wicked problems in design thinking. Design Issues, 8(2), 5-21.

Evans, V. (2004). The structure of time: Language, meaning, and temporal cognition. Amsterdam: John Benjamins.

Grabinger, S. (2004). Design lessons for social education. In T. M. Duffy \& J. R. Kirkley (Eds.), Learner-centered theory and practice in distance education: Cases from higher education (pp. 49-60). Mahwah, NJ: Lawrence Erlbaum.

Mehlenbacher, B. (2010). Instruction and technology: Designs for everyday learning. Cambridge, MA: MIT Press.

\section{Evidence-Based eLearning}

- Web-Based Experiment Control for Research on Human Learning

\section{Evidence-Based Learning}

\section{Jacquelyn Cranney, Fiona McDonald}

School of Psychology, University of New South Wales, Sydney, NSW, Australia

\section{Synonyms}

Effective learning

\section{Definition}

Learning is a lifelong process and both individuals and organizations are concerned with evidence for what makes "good learning." Evidence-based learning describes a class of approaches, processes, and strategies that have been empirically demonstrated to produce learning outcomes. This definition subsumes another possible definition - that of "evidence that learning has occurred" - which assumes that learning can be measured. For the purposes of this volume, learning involves those effortful strategies undertaken by students to meet the requirements of their enrollment in a formal educational institution, with a formal 
end-point being graduation from that institution with a specific qualification. In this context, students are learners; instructors, teachers and educators are those professionals who facilitate students' learning; and learning outcomes (such as knowledge, skills and professional dispositions) are the products of learning. Learning outcomes are usually measured by formal assessment tasks such as examinations, written assignments, and skills tests. Empirically based principles for improving the effectiveness of learning are relevant to evidence-based learning. Educators can apply these principles in classrooms to improve learning by students, and students can apply these principles both in class and in their personal study time to improve their learning and thus their success in achieving their educational goals. It should be noted that "empirically based principles" are derived from systematic observation and hypothesis testing, characteristic of a scientific approach, and that an "evidence-based" procedure means that a procedure has been found to be effective through the application of at least comparison-group scientific methodology.

\section{Theoretical Background}

It should be noted that the term "evidence-based learning" has been utilized to describe specific techniques in classrooms, which in the tradition of educational research and practice, has been subject to criticism and countercriticism (e.g., Burton and Underwood 2000). The broader definition of evidence-based learning given above is nevertheless pragmatically restrictive: learning does, of course, occur in contexts (formal and informal) other than the actual or virtual classrooms of educational institutions. Nevertheless, the latter is where research and government funding is focused, and from where the audience for this volume is likely to be drawn. Even with that boundary condition, education is a diverse enterprise, attracting research attention and different epistemological approaches from a number of disciplines, including education, sociology, and psychology. In this brief section, three examples of different theoretical approaches to the (usually implicit) concept of "evidence-based learning" are presented.

From a sociological educational perspective, Hodkinson (2008) criticized the UK government policy-driven audit and evidence-based approach to assessing the "efficiency" of educational practice in the UK. He argues that this approach: (a) emphasizes accountability to government and consumers, this being based on a mistrust of professional bodies which are seen as promoting their own self-interests, (b) misrepresents and misunderstands the concept of "learning" simply as acquisition of knowledge that eventually results in a qualification, (c) assumes that "learning" performance indicators such as retention and completion/achievement of qualification are primarily attributable to the effectiveness of educational strategies, and are thus reflective of the competence of individual teachers and the efficiency of institutional systems, and (d) assumes that general principles can be derived from positivistic educational research that can be applied equally well to improving "learning" across all contexts (and is thus subservient to educational policy in a resource-poor sector). Hodkinson (2008) counterargues that this approach is too simplistic, pointing out, for example, that there are a wide variety of reasons why students drop out of courses, that have little to do with the educator or the institution. Moreover, he presents evidence for long-term adverse effects of the audit culture on student learning. He counters that: (a) learning need not be judged only for its effectiveness but for its value, (b) there are many different positive learning processes and outcomes, (c) improving the effectiveness of learning involves modifying - learning cultures, rather than imposing rigid standard practices, (d) learning cultures can be improved by facilitating localized initiative, creativity, and professional judgment, and rewarding such, and (e) improving learning requires a "reflectively critical understanding at all levels of intervention: government, college, tutor and, where possible, student" (p. 319). Essentially, Hodkinson is arguing that the audit culture (whereby whole institutions are evaluated) and associated research has not led to improved student-learning outcomes at the classroom level.

The role of the student in learning is emphasized by Mayer (1992) in his examination of the relationship between psychology and instruction, where he traces the history of educational psychology from behaviorist to cognitive to constructivist approaches. With the latter, he states that "the learner changed from that of a recipient of knowledge to that of a constructor of knowledge, an autonomous learner with metacognitive skills for controlling his or her cognitive processes during learning ....[and] learning involves selecting relevant 
information and interpreting it through one's existing knowledge" (p. 407). Thus, learners are active processors of information, learning is the construction of knowledge by learners, and the central instructional issue is how to help students become more effective processors of information. The products of learners' characteristics and learning processes are learning outcomes, which are the "internal cognitive structures constructed during learning," and "outcome performance" is the external performance of learners on tests (p. 408). With this approach, then, "evidencebased learning" may be conceptualized as involving strategies (taking into account factors such as students' characteristics) that optimize learning outcomes, as measured with assessment tasks.

Worrell et al. (2010) recently proposed a dynamic process model of teaching and learning that conceptualizes teachers and students as consumers of, as well as contributing to, evidence-based learning. Indeed, they argue that both students and teachers should be professional (and thus ethical) in their approach to learning, and in particular they need to regularly evaluate those approaches. In Worrell et al.'s model, educators start by (a) researching what empirically based principles, derived mostly from laboratory but also increasingly from classroom research, could be translated into practice in their particular course, and (b) considering what did, and did not, "work" in their previous delivery of that course. Educators then decide what strategies to employ on the basis of their judgment of what will lead to the most effective learning for those particular students, with that particular subject matter, given the particular context (including available resources). This constitutes "scholarly teaching," and if educators then communicate their experiences in scholarly contexts, this then transforms to the scholarship of learning and teaching, which contributes to the "evidence base" of promising principles for effective learning. It should be noted that the contextual factors influencing translation to practice can be related to Hodkinson's (2008) concept of "learning culture." Meanwhile, students begin a course with (a) expectations as to its nature, (b) certain motivations regarding what they hope to achieve in that course (goals), and (c) the learning strategies that they will utilize to achieve those learning goals - all of which may change as they experience the course. In Worrel et al.'s dynamic process model, a student engages in " order to achieve learning goals. At the heart of this model is the educator-learner interaction. A goal of an educator should include the explicit improvement of students' self-regulated learning, to improve the likelihood that learning does occur. For example, the educator could explicitly point out the advantages of strategies such as concept mapping in classroom exercises, and how these can be easily transferred to students' personal study strategy repertoires (Worrell et al. 2010).

Worrell et al. (2010) outline some principles derived primarily from laboratory-based research that could be transferred to a classroom situation as part of scholarly teaching by the educator, or to self-regulated learning by the student. These include the notions of desirable difficulties, deep explanatory processing, organization effects, spaced learning over time, testing as a teaching device, metacognition, transfer appropriate processing to new contexts, and teacher and learner as holistic agents. For example, Robert Bjork's “desirable difficulties" acknowledges that most learning of value in formal educational contexts is effortful; that is, it takes work to gain meaningful knowledge, skills, and professional dispositions (Worrell et al. 2010). A simple example is requiring students to generate answers based on scaffolding, rather than the teacher providing all the answers (Worrell et al. 2010). Note that this notion is contrary to the idea that "good" teaching practices necessarily make learning "easy." The principle of spaced learning over time being superior to massed learning was derived long ago from laboratory research with animals, and has been supported empirically by human research both in the laboratory and in classrooms; nevertheless, this principle rarely appears to be put into practice by educators (Worrell et al. 2010). Recently reinvigorated research strongly supports the value of formative testing as a teaching device which improves learning as indexed by summative assessments (Worrell et al. 2010), and this strategy can be easily adopted by students in their personal study-time. Finally, the concept of metacognition fully acknowledges the role of students as knowledge constructors (Mayer 1992). Overall, Worrell et al. (2010) emphasize that the process of learning reflects a dynamic ongoing interaction between learners, educators, and context, and that although research into effective learning should take these complexities into account, it is possible for individual learners and 
educators to be "personally empirical" (p. 135) in their approach to effective learning.

\section{Important Scientific Research and Open Questions}

Hodkinson's (2008) critique of the auditing of learning outcomes seriously challenges the appropriateness of current attempts to standardize and assess learning outcomes. More importantly for the issue at hand, he criticizes the "one-size-fits-all" research approach to improving learning outcomes. However, Mayer (1992) and Worrell et al. (2010) argue convincingly that current educational psychology research does take into account contextual factors, and that further research on the translation of psychological science to the optimization of learning in different educational contexts is clearly required. In doing so, it is important not to forget the central role of students and all their complexities, including their high- or low-level learning goals, and their capacity to learn about how best to learn in a given situation. Some current issues in need of consideration are presented below:

- Some educators argue that learning is better measured in real-life situations than through in-course assessments such as examinations. However, it may then be challenging to assess the effectiveness of a particular learning strategy; nevertheless, the notions of (a) work-integrated learning assessment and (b) post-graduation assessment should be explored.

- There is a risk that teaching and learning methods are unduly influenced by trends, labeling, and surface features. Using evidence-based learning should minimize these risks. Research assessing this assertion is required, particularly given the audit culture.

- The quality of evidence for strategies that improve learning outcomes needs to be examined from the perspective of the quality of the methodology used (and thus the inferences that are possible).

- As Worrell et al. (2010) argue, much more translational research is required to test the application of psychological principles to the facilitation of classroom learning.

\section{Cross-References}

- Approaches to Learning and Study

- Assessment of Learning
- Effects of Testing on Learning

- Learning About Learning

- Learning Strategies

- Metacognition and Learning

- Self-regulated Learning

\section{References}

Burton, J. L., \& Underwood, J. C. E. (2000). Evidence-based learning: A lack of evidence-letter to the editor. Medical Teacher, 22, 136-140.

Hodkinson, P. (2008). Scientific research, educational policy and educational practice in the United Kingdom: the impact of the audit culture on further education. Cultural Studies - Critical Methodologies, 8, 303-324.

Mayer, R. E. (1992). Cognition and instruction: Their historic meeting within educational psychology. Journal of Educational Psychology, 84, 405-412.

Worrell, F. C., Casad, B. J., Daniel, D. B., McDaniel, M., Messer, W. S., Miller, H. L., Prohaska, V., \& Zlokovich, M. S. (2010). Promising principles for translating psychological science into teaching and learning. In D. F. Halpern (Ed.), Undergraduate education in psychology: A blueprint for the future of the discipline (pp. 129144). Washington, DC: American Psychological Association.

\section{Evidence-Based Teaching}

- Behavioral Approaches to Instruction

\section{Evolution and Memory}

- Adaptive Memory and Learning

\section{Evolution of Learning}

Mauricio R. PAPINI

Department of Psychology, Texas Christian University, Fort Worth, TX, USA

\section{Synonyms}

Comparative psychology of learning; Evolution of memory

\section{Definition}

Studies on the evolution of learning use a comparative perspective to understand the ability of organisms to 
acquire, consolidate, and retrieve information originating in experience. These processes are tested in behavioral assays, and the organism's ability to adjust to particular situations is often related to the nature of the situation, internal conditions, prior experience, and the species' ecological and phylogenetic characteristics. These studies open two main problems for analysis: (1) What evolutionary hypotheses help explain species similarities in learning phenomena? (2) What evolutionary hypotheses help explain species differences in learning phenomena? Comparative studies lead to an understanding of the diversity of mechanisms that have evolved to deal with the ability of learning from experience - a remarkable capacity of neural tissue.

\section{Theoretical Background}

Scientists interested in the evolution of learning mechanisms ask two fundamental questions:

- Are species similarities understandable in terms of the evolutionary homology or homoplasy of learning mechanisms?

- Are species differences in learning attributable to the evolutionary divergence of learning mechanisms?

Homology, homoplasy, and divergence are evolutionary outcomes. Divergence refers to the split of lineages with regard to a particular trait (e.g., grasping feet of chimpanzees vs. flat feet of humans). Homology refers to phenotypic similarity attributable to common ancestry (e.g., all primates have nails). Homoplasy refers to phenotypic similarity attributable to independent evolution in response to similar environmental pressures (e.g., similar body shape of sharks and whales). Applying these concepts to the study of learning requires recognizing a distinction between learning phenomena and learning mechanisms. Learning phenomena are typically induced under laboratory conditions and involve a comparison between experimental and control groups. Learning phenomena must be the result of learning mechanisms - a set of processes that account for the behavioral phenomenon.

What is a learning mechanism? There are at least four levels of mechanistic analysis in the study of learning (Papini 2002):

- Psychological level: The traditional level of analysis developed by comparative psychologists.
Methodology: dissection of effects through experimental design contrasting different training conditions. Psychological concepts offer a guide to interpret the effects of manipulations at lower levels of analysis on behavior (Bitterman and Woodard 1976).

- Neurobiological level: Specifying the neural network necessary to produce a given learning phenomenon. Methodology: Lesion and stimulation of restricted brain regions to determine their role in a given learning phenomenon.

- Neurochemical level: Synaptic processes, including synaptic plasticity, implicated in learning. Methodology: Administration of psychoactive drugs and genetic manipulations (e.g., knockout strains) affecting synaptic transmission.

- Cell-molecular level: Cellular cascades, including gene expression and protein synthesis, necessary for synaptic plasticity during learning. Methodology: Drug and genetic manipulations that affect cellular processes involved in synaptic plasticity.

According to this view of learning mechanisms (Papini 2002), a homology hypothesis is strengthened if a learning phenomenon occurring in two or more species depends on the same processes at all four levels of analysis. By contrast, a homoplasy hypothesis is strengthened if similar learning phenomena across species depend on different processes at one or more levels of analysis. Finally, a divergence hypothesis is favored when learning phenomena are shown to depend on different mechanisms at one or more levels of analysis. In all cases, the alternative hypothesis is that similarities or differences in learning phenomena are caused by species differences in contextual mechanisms: sensory-perceptual, motivational, and motor processes (Bitterman 1975). Because the role of contextual variables is difficult to dismiss completely, the conclusions drawn in the next section are expressed in tentative terms.

\section{Important Scientific Research and Open Questions}

\section{Species Similarities in Learning Mechanisms}

The simplest nervous systems are the diffuse neural networks of radially symmetrical animals, such as sea 
anemones, coral, and jellyfish (Cnidaria). Cnidarian neurons exhibit many of the physiological properties found in neurons from other animals, including mammals (Anderson 1985). Behaviorally, cnidarians also show familiar learning phenomena. Classical conditioning was reported in sea anemone (Cribrina xanthogrammica) exposed to pairings of light and electric shock (Haralson et al. 1975). Although classical conditioning is within the capacity of radially symmetrical animals with diffuse neural networks, the underlying mechanisms have not yet been identified.

Most animals exhibit a bilateral body plan and a distinction between central and peripheral nervous systems. Classical conditioning has also been reported in experiments with simple bilateral animals, including planaria (Platyhelminthes; Baxter and Kimmel 1963; Jacobson et al. 1967), leeches (Annelida; Sahley and Ready 1988), and the round worm Caenorhabditis elegans (Nematoda; Morrison et al. 1999; Wen et al. 1997). C. elegans is interesting because behavioral studies can be combined with physiological and genetic manipulations of learning enabled by the striking simplicity of this animal (Giles et al. 2006). The central nervous system of $C$. elegans contains 302 neurons, all identified and with a known connectivity, and its genome has been mapped. These studies confirm that a simple neural circuit can be sufficient for classical conditioning.

Learning has also been studied in mollusks. Cephalopods have traditionally been considered behaviorally and neurologically complex invertebrates (Mather 2006). Classical conditioning has been demonstrated in cuttlefish (Sepia officinalis; Purdy et al. 1999) and octopuses (Octopus cyanea; Papini and Bitterman 1991), rapid spatial learning was reported in O. bimaculoides (Boal et al. 2000), and problem solving in O. vulgaris (Fiorito et al. 1990). Classical conditioning has also been documented in gastropod mollusks like Aplysia californica (Carew et al. 1983; Colwill et al. 1997) and the terrestrial snail Limax maximum (Sahley et al. 1981).

The complex sensory systems and refined behavior of insects (Arthropoda) allow for elegant demonstrations of learning rivaling those involving vertebrates. Honeybees (Apis mellifera; Bitterman 1988, 1996; Prete 2006) trained to respond to visual, olfactory, and magnetic stimuli have produced a range of learning phenomena familiar from research with vertebrates. From a comparative perspective, this remarkable generality across insects and mammals can be understood in terms of deep homology, massive convergence, or parallel evolution of learning mechanisms (Papini 2008). Deep homology can be dismissed on the assumption that the nervous systems of insects and mammals have evolved largely independent from each other (although invertebrate and vertebrate nervous systems do share some molecular markers; see BenitoGutierrez and Arendt 2009). For example, there is no area homologous to the vertebrate amygdala in insects. Thus, whereas neurons may have the same properties, the systems, qua systems, have evolved independently. To the extent that some forms of learning require specific neural architectures (i.e., the neurobiological level), similarities in learning mechanisms among animals from diverse phyla must be homoplasic.

Homoplasy is consistent with either massive convergence, which would be supported by different underlying mechanisms at all levels, or parallel evolution, which would be consistent with homologies at the lower levels of analysis. Available evidence points to parallel evolution based on homologous cell-molecular processes. One hypothesis is that cellular processes involved in synaptic plasticity were established early in animal phylogeny and are thus homologous across many species. One candidate for cell-molecular homology involves second-messenger systems engaged by repeated activation of a synaptic pathway (e.g., cyclic adenosine-monophosphate, cAMP). Second messengers can induce cellular changes mediated by gene expression, such as the up-regulation or downregulation of synaptic receptors (Bailey and Kandel 2009). Such cellular correlates of learning have been described in Aplysia (mollusk), Drosophila (insect), and rodents (vertebrate) (Kaplan and Abel 2003; Mayford and Kandel 1999; McGuire et al. 2005).

\section{Species Differences in Learning Mechanisms}

Comparative studies of differences in learning mechanisms emphasize either adaptive significance (Domjan 1997) or phylogenetic history (Bitterman 1975). Consider flavor aversions as an example of adaptive learning. In flavor aversions (Garcia and Koelling 1966; Miller and Domjan 1981), rats exposed to pairings between a taste or an audiovisual stimulus and either X-ray irradiation (which induces gastrointestinal intoxication) or electric shock (which causes peripheral 
pain) later exhibited a type of selectivity in their behavior. Rats seemed to learn more about the taste when this was paired with intoxication than with pain, but more about the audiovisual stimulus when it was paired with pain rather than intoxication. Such selectivity has been interpreted as representing adaptive specializations. For example, rats learn flavor aversions because they forage for food using gustatory and olfactory cues, whereas bobwhite quail (Colinus virginianus) learn visual aversions because they find food on the basis of visual cues (Wilcoxon et al. 1971). However, studies with closely related species with different feeding ecology suggest that contextual factors (e.g., neophobia) could play a major role in flavor aversions (Daly et al. 1982).

Consider successive negative contrast (SNC) as an example of divergence related to phylogenetic history. In SNC, an animal exhibits greater rejection of a small incentive after being downshifted from a large incentive, than an animal always trained with the small incentive. SNC has been found in mammals (e.g., Papini et al. 1988), but not in teleost fish trained under analogous conditions (e.g., Lowes and Bitterman 1967). A similar distinction between mammals and teleost fish also appears in other learning phenomena also involving incentive downshifts (Papini 2003, 2006). Despite this apparent phylogenetic divergence, it is still possible that SNC may be eventually demonstrated in teleost fish using novel training procedures.

Behavioral plasticity with obvious adaptive significance can be produced in a variety of ways. Natural selection can affect sensory-perceptual, motivational, and motor processes, in addition to learning mechanisms, to fine-tune adaptive behavior. The fact that adaptive behavior is often achieved by evolutionary changes in non-learning factors suggests rigid developmental constraints that limit the evolutionary modification of learning mechanisms. One possible advantage of adjusting contextual mechanisms, rather than learning mechanisms, is that the needed genetic changes would have relatively restricted phenotypic effects.

\section{Cross-References}

- Adaptive Learning Through Variation and Selection

- Animal Learning and Intelligence

- Animal Perceptual Learning

- Associative Learning

- Neural Networks of Classical Conditioning

\section{References}

Anderson, P. A. V. (1985). Physiology of a bidirectional, excitatory chemical synapse. Journal of Neurophysiology, 53, 821-835.

Bailey, C. H., \& Kandel, E. R. (2009). Synaptic and cellular basis of learning. In G. G. Berntson \& J. T. Cacioppo (Eds.), Handbook of neuroscience for the behavioral sciences (pp. 528-551). Hoboken: Wiley.

Baxter, R., \& Kimmel, H. D. (1963). Conditioning and extinction in the planarian. The American Journal of Psychology, 76, 665-669.

Benito-Gutierrez, E., \& Arendt, D. (2009). CNS evolution: New insight from the mud. Current Biology, 19, R640-R642.

Bitterman, M. E. (1975). The comparative analysis of learning. Science, 188, 699-709.

Bitterman, M. E. (1988). Vertebrate-invertebrate comparisons. In H. J. Jerison \& I. Jerison (Eds.), Intelligence and evolutionary biology (pp. 251-275). Berlin: Springer.

Bitterman, M. E. (1996). Comparative analysis of learning in honeybees. Animal Learning \& Behavior, 24, 123-141.

Bitterman, M. E., \& Woodard, W. T. (1976). Vertebrate learning: Common processes. In R. B. Masterton, C. B. G. Campbell, M. E. Bitterman, \& N. Hotton (Eds.), Evolution of brain and behavior in vertebrates (pp. 169-189). Hillsdale: Erlbaum.

Boal, J. G., Dunham, A. W., \& Williams, K. T. (2000). Experimental evidence for spatial learning in octopuses (Octopus bimaculoides). Journal of Comparative Psychology, 114, 246-252.

Carew, T. J., Hawkins, R. D., \& Kandel, E. R. (1983). Differential classical conditioning of a defensive withdrawal reflex in Aplysia californica. Science, 219, 397-400.

Colwill, R. M., Goodrum, K., \& Martin, A. (1997). Pavlovian appetitive discriminative conditioning in Aplysia californica. Animal Learning \& Behavior, 25, 268-276.

Daly, M., Rauschenberger, J., \& Behrends, P. (1982). Food aversion learning in kangaroo rats: A specialist-generalist comparison. Animal Learning \& Behavior, 10, 314-320.

Domjan, M. (1997). Behavior systems and the demise of equipotentiality: Historical antecedents and evidence from sexual conditioning. In M. E. Bouton \& M. S. Fanselow (Eds.), Learning, motivation, and cognition (pp. 31-51). Washington, DC: American Psychological Association.

Fiorito, G., von Planta, C., \& Scotto, P. (1990). Problem-solving ability of Octopus vulgaris Lamarck (Mollusca, Cephalopoda). Behavioral and Neural Biology, 53, 217-230.

Garcia, J., \& Koelling, R. A. (1966). Relation of cue to consequence in avoidance learning. Psychonomic Science, 4, 123-124.

Giles, A. C., Rose, J. K., \& Rankin, C. H. (2006). Investigations of learning and memory in Caenorhabditis elegans. International Review of Neurobiology, 69, 37-71.

Haralson, J. V., Groff, C. I., \& Haralson, S. J. (1975). Classical conditioning in the sea anemone, Cribrina xanthogrammica. Physiology \& Behavior, 15, 455-460.

Jacobson, A. L., Horowitz, S. D., \& Fried, C. (1967). Classical conditioning, pseudoconditioning, or sensitization in the planarian. Journal of Comparative and Physiological Psychology, 64, 73-79.

Kaplan, M. P., \& Abel, T. (2003). Genetic approaches to the study of synaptic plasticity and memory storage. CNS Spectrums, 8, 597-610. 
Lowes, G., \& Bitterman, M. E. (1967). Reward and learning in the goldfish. Science, 157, 455-457.

Mather, J. A. (2006). Behaviour development: A cephalopod perspective. International Journal of Comparative Psychology, 19, 98-115.

Mayford, M., \& Kandel, E. R. (1999). Genetic approaches to memory storage. Trends in Genetics, 15, 463-470.

McGuire, S. E., Deshazer, M., \& Davis, R. L. (2005). Thirty years of olfactory learning and memory research in Drosophila melanogaster. Progress in Neurobiology, 76, 328-347.

Miller, V., \& Domjan, M. (1981). Specificity of cue to consequence in aversion learning in the rat: Control for US-induced differential orientations. Animal Learning \& Behavior, 9, 339-345.

Morrison, G. E., Wen, J. Y. M., Runciman, S., \& van der Kooy, D. (1999). Olfactory associative learning in Caenorhabditis elegans is impaired in lrn-1 and lrn-2 mutants. Behavioral Neuroscience, 113, 358-367.

Papini, M. R. (2002). Pattern and process in the evolution of learning mechanisms. Psychological Review, 109, 186-201.

Papini, M. R. (2003). Comparative psychology of surprising nonreward. Brain, Behavior and Evolution, 62, 83-95.

Papini, M. R. (2006). Role of surprising nonreward in associative learning. Japanese Journal of Animal Psychology, 56, 1-20.

Papini, M. R. (2008). Comparative psychology. Evolution and Development of Behavior (2nd ed.). New York: Psychology Press.

Papini, M. R., \& Bitterman, M. E. (1991). Appetitive conditioning in Octopus cyanea. Journal of Comparative Psychology, 105, 107-114.

Papini, M. R., Mustaca, A. E., \& Bitterman, M. E. (1988). Successive negative contrast in the consummatory responding of didelphid marsupials. Animal Learning \& Behavior, 16, 53-57.

Prete, F. R. (Ed.). (2006). Complex learning in arthropods. International Journal of Comparative Psychology, 19(3) (special issue).

Purdy, J. E., Roberts, A. C., \& Garcia, C. A. (1999). Sign tracking in cuttlefish (Sepia officinalis). Journal of Comparative Psychology, 113, 443-449.

Sahley, C. L., \& Ready, D. F. (1988). Associative learning modifies two behaviors in the leech, Hirudo medicinalis. The Journal of Neuroscience, 8, 4612-4620.

Sahley, C. L., Rudy, J. W., \& Gelperin, A. (1981). An analysis of associative learning in a terrestrial mollusc. I. Higher-order conditioning, blocking, and a transient US pre-exposure effect. Journal of Comparative Physiology A, 144, 1-8.

Wen, J. Y. M., Kumar, N., Morrison, G., Rambaldini, G., Runciman, S., Rousseau, J., \& van der Kooy, D. (1997). Mutations that prevent learning in C. elegans. Behavioral Neuroscience, 111, 354-368.

Wilcoxon, H. C., Dragoin, W. B., \& Kral, P. A. (1971). Illness-induced aversions in rat and quail: Relative salience of visual and gustatory cues. Science, 171, 826-828.

\section{Evolution of Networks}

Networks, Learning Cognition, and Economics

\section{Evolutionary Computation}

An area of artificial intelligence in which an artificial simulation of Darwinian evolution is used to evolve solutions to problems. Types of evolutionary algorithms include genetic algorithms, genetic programming, and evolution strategies. These algorithms share a number of traits: (a) a population of chromosomes or data records that denote candidate solutions; (b) a fitness function or objective function that assigns a score of merit to a candidate solution; (c) the ability to take chromosomes and generate offspring, usually by using crossover (genetic exchange of material between chromosomes), and mutation (random alteration of chromosome values); and (d) fitness-based selection (stronger chromosomes are more likely to be selected for reproduction). Evolutionary algorithms can be considered to be optimization algorithms (they attempt to find the optimal solution, if possible), search algorithms (they search for a solution exhibiting the desired traits), randomized algorithms (many aspects of the algorithm rely on pseudo-randomization, for example, to create initialized populations), and constructive solution generators (they assemble solutions from building blocks).

\section{Cross-References}

- Self-Adaptation of Cultural Learning Parameters

\section{Evolutionary Dynamics, Collective Action}

\author{
- Learning Dynamics in Social Dilemmas
}

\section{Evolution of Memory}

\section{Evolutionary Educational Psychology}

- Evolution of Learning

- Adaptability and Learning 


\section{Evolutionary Epistemology}

Naturalistic Epistemology

\section{Evolutionary Learning and Stochastic Process Algebra}

BRIAN J. Ross

Department of Computer Science, Brock University, St. Catharines, ON, Canada

\section{Synonyms}

Genetic programming; Stochastic pi-calculus

\section{Definition}

Here, evolutionary learning refers to the use of evolutionary computation to find solutions to problems. Evolutionary computation is a branch of artificial intelligence that focuses on using an artificial simulation of natural evolution based on Darwinian natural selection. The goal is to use artificial evolution as a means for finding an acceptable solution to a problem of interest. Evolutionary computation has been used as a problem solving strategy for countless problems in various disciplines. The application of concern here is the use of genetic programming to synthesize stochastic models written in stochastic process algebra. Genetic programming is a form of genetic algorithm, in which program code or mathematical formulae are subjected to evolution. Some advocates claim that genetic programming is an instance of automatic programming, in which computer systems automatically design their own program code with minimal human influence. Stochastic process algebras are used in the field of systems biology as a formal language for modeling and simulating biological systems. Research is investigating the use of genetic programming to automate the creation of biological network models as written in stochastic process algebra.

\section{Theoretical Background}

The stochastic pi-calculus is a type of process algebra (Priami 1995). It is a mathematical language effective for modeling many kinds of phenomena in natural sciences, for example, networks of biochemical reactions. The behaviors of these models are characterized by the time-series output values they generate, which denote changing quantities of various proteins or chemicals over time. These time series often exhibit variable, chaotic behaviors, which naturally arise within the stochastic semantics of the algebra. However, process algebra are highly technical to learn and master, and especially so for those working in unrelated disciplines such as biology. Their challenging nature suggests that their automatic construction by machine learning techniques is worth considering. This would result in the automatic construction of biological models that explain observed laboratory data. This idea is related to other research in bioinformatics, in which machine learning is applied to problems in biology; Bayesian network learning and neural network learning are two popular approaches (Baldi and Brunak 1998). Also pertinent to this are techniques for modeling time-series data, which has been a subject of active study for centuries.

Genetic programming is an active research area, and it has been applied to many diverse problems. Two preliminary requirements for applying genetic programming to a new problem are: (a) potential solutions to the problem in question can be evaluated by the computer and assigned suitable numerical scores of merit and (b) a computer-executable target language capable of representing solutions to the problem exists. The challenge of applying genetic programming to the stochastic pi-calculus arises from the algebra's fragility when expressions are manipulated during evolution. This results in a difficult "search space" for evolution to navigate and, naturally, prohibits the discovery of high quality solutions. Furthermore, characterizing the time-series behavior of a desired stochastic solution can be very difficult, which is an inherent problem when dealing with stochastic time series in general. Finally, genetic programming is one of the more computationally expensive machine learning paradigms. Since stochastic pi-calculus simulations are usually computationally intensive as well, the combination of these systems together may be problematic.

\section{Important Scientific Research and Open Questions}

Genetic programming has successfully evolved smallscaled stochastic pi-calculus processes (Ross and Imada 
2009). The implementation of the language within the genetic programming system used grammatical constraints to refine the form of solutions constructed. In addition, stochastic time-series behaviors were represented with multiple statistical feature analyses, which are commonly used elsewhere for modeling complex time series. A simple example of such a feature might be the mean value seen over the duration of a time series. Moderately larger examples were evolved after using multi-objective evaluation strategies, which are effective for many problems having independent scoring measurements (Ross and Imada 2011). In this case, the various statistical features of different timeseries curves are treated as separate objectives.

The main open question in this area of research is the extent to which this methodology can scale up to larger, more interesting and realistic biological networks. Further advances will require continued research in a number of issues. Firstly, the basic stochastic pi-calculus will unlikely be a practical target for large-scale models within a genetic programming framework, unless more advanced grammatical constraints are introduced. Otherwise, the search space will remain difficult and largely intractable for large problems. Secondly, advances in the area of feature selection can greatly help in determining optimal sets of effective statistical features to use and to denote target behaviors of interest.

Recently, substantial advances were made by applying genetic programming to higher-level stochastic languages. Considerably more complex models were evolved by considering higher-level stochastic languages that are implemented on top of the stochastic pi-calculus (Imada and Ross 2010; Ross 2010). These languages define more tractable, evolutionary friendly search spaces than the raw stochastic pi-calculus.

\section{Cross-References}

- Bayesian Learning

- Causal Learning

- Computational Learning Theory

- Learning in Artificial Neural Networks

- Stochastic Learning Automata-Based Time Series Analysis

\section{References}

Baldi, P., \& Brunak, S. (1998). Bioinformatics: The machine learning approach. Cambridge, MA: MIT Press.
Imada, J., \& Ross, B. J. (2011). Evolutionary synthesis of stochastic gene network models using feature-based search spaces. New Generation Computing, 29(4).

Priami, C. (1995). Stochastic pi-calculus. The Computer Journal, $38(7), 579-589$.

Ross, B. J. (2010). The evolution of higher-level biochemical reaction models.

Ross, B. J., \& Imada, J. (2009). Evolving stochastic processes using feature tests and genetic programming. In: Proceedings genetic and evolutionary computation conference 2009, Montreal.

Ross, B. J., \& Imada, J. (2010). Using multi-objective genetic programming to synthesize stochastic processes. In R. Riolo, U.-M. O'Reilly, \& T. McConaghy (Eds.), Genetic programming theory and practice VII (pp. 159-175). New York: Springer.

\section{Evolutionary Learning in Networks}

Naïve Learning in Networks

\section{Evolutionary Psychology}

Adaptability and Learning

\section{Evolutionary Robotics}

- Cognitive Modeling with Multiagent Systems

- Robot Learning Via Human-Robot Interaction: The Future of Computer Programming

\section{Evolved Modules}

Folk Knowledge and Academic Learning

\section{Exact Learning}

Query-Directed Learning 


\section{Examination Stress and Components of Working Memory}

\author{
Richard S. Lewis, Nicole Y. Weekes, Natalie Guerrero \\ Departments of Psychology and Neuroscience, \\ Pomona College, Claremont, CA, USA
}

\section{Synonyms}

Executive control; Naturalistic stressor; Short-term memory

\section{Definitions}

Examination stress represents a type of naturalistic stressor known to elevate psychological markers, such as self-reported negative affect, and physiological markers, such as cardiovascular activity and the production of a number of stress-related hormones. Typically, examination stress studies involve measuring the stress response during a period of a high stakes examination, such as a medical school licensing exam, or during a period of multiple exams, as in a week of undergraduate final exams. Measures from these examination periods are then compared with periods preceding or subsequent to the exam.

Working memory refers to the neural-cognitive system involved in the temporary storage and manipulation of information, or a "mental workspace." The prefrontal cortex is part of a distributed neural system subserving working memory and is most critically involved in the manipulation of information or executive component of working memory (D'Esposito 2007). Additional components of working memory are involved in temporarily retaining material-specific, task-relevant information. Parcing the components of working memory has been a major research focus, with most attention given to characterizing the verbal and visual components of working memory, along with the executive component. Working memory has been identified as a critical element of reasoning, problem solving, and intelligence. Therefore, understanding how examination stress affects working memory is an important practical issue, as well as providing a useful naturalistic model for studying stress in real-world situations.

\section{Theoretical Background}

One critical effect of stressor exposure is the release of glucocorticoids, a type of steroid hormone released from the adrenal glands. Cortisol, a prominent glucocorticoid in humans, acts on mineralcorticoid receptors (MR) and glucocorticoid receptors (GR) in the central nervous system, and is thought to mediate many of the important cognitive and behavioral changes associated with stress. High concentrations of glucocorticoid receptors are found in the amygdala, hippocampus, and the frontal lobes. The prefrontal cortex serves as a major negative feedback loop of the hypothalamic-pituitary-adrenal axis and, in doing so, down-regulates subsequent glucocorticoid release. Because the frontal lobes are also known to play an important role in working memory, it is not surprising that the binding of cortisol to the plentiful glucocorticoid receptors in the prefrontal cortex should be found to alter aspects of working memory.

In 1908, Yerkes and Dodson described a curvilinear relationship between shock intensity and visual discrimination in rodent performance on a visual discrimination task. This relationship has become known as the Yerkes-Dodson Law and is conceptualized to describe an inverted $U$ relationship between arousal and performance. That is, there is an optimal level of arousal for peak performance, and too low or too high levels of arousal are associated with suboptimal performance. Both working and declarative memory have been shown to be influenced by stress, and, in both cases, there is evidence to suggest an inverted $\mathrm{U}$ function. That is, moderate levels of stress tend to be associated with optimal memory performance. While a number of theories exist to explain this curvilinear relationship, one of the most prominent suggests that low to moderate levels of stress are associated with glucocorticoid binding to mineralocorticoid receptors, which is associated with improved memory performance, whereas relatively high levels of stress are associated with a saturation of MR receptors and glucocorticoid binding to glucocorticoid receptors (GR). This GR binding is associated with impaired memory performance (Lupien et al. 1999).

In general, three approaches have been taken to experimentally studying stress in humans. The first involves hydrocortisone administration, the second involves exposure to laboratory stressors, such as the Trier Social Stress Test (TSST), a standardized protocol 
of public speaking and arithmetic designed to induce stress in a laboratory setting, and the third involves exposure to naturalistic stressors such as examination stress. While naturalistic stressor studies are used least often, they may provide better external validity. That is, it is not clear how well the stress response triggered by either drug administration or laboratory stressors mimics that observed in the natural environment. For example, the elevation in cortisol release can range from $100 \%$ to $200 \%$ in the case of hydrocortisone administration and from $50 \%$ to $100 \%$ in the case of the TSST. In contrast, the elevation in cortisol release following examination stress tend to be far more modest, and in the range of $20-40 \%$. Therefore, it remains unclear the extent to which reactions to "nonnaturalistic" stressors may be externally valid and generalizable to naturally occurring settings.

\section{Important Scientific Research and Open Questions}

Generally, the stress of examinations elicits elevated activity in the HPA axis and increased release of cortisol (Weekes et al. 2006). However, there is some inconsistency in the literature. One explanation for the inconsistent findings is that the extent to which a stressor (including examinations) triggers an elevation in cortisol is dependent on a variety of factors, including novelty, uncertainty, negative emotions, and egoinvolvement. While the last decade has evidenced a significant broadening in the studies that utilize examination stress as the stressor, usage of the protocol extends back far further in the field of psychoneuroimmunology (PNI).

Within the field of PNI, there is clear evidence that examination stress alters immune functioning. For example, examination stress has been found to alter the level of activity of a number of immune cells (e.g., B- and T-Lymphocytes) and messenger chemicals, all critical to the proper functioning of the immune system. More specifically, some of the strongest evidence suggest that examination stress alters levels of immunoglobulin A (S-IgA), an antibody that plays a role in mucosal immunity and respiratory health, as well as levels of natural killer cells (NK cell), which are critical to preventing tumurous growths. However, the extent to which these stress-related alterations in immunity directly affect specific health outcomes remains an open, and highly debated, question.
The relationship between stress and memory depends on the intensity of stress experienced, the specific process of memory measured, and the difficulty of the memory challenge. For example, there is general agreement in the literature that stress is associated with an improvement in declarative memory consolidation and an impairment in declarative memory retrieval (Roozendaal 2002), and these relationships are observed whether stress is measured psychologically or hormonally. However, the literature on working memory is more mixed. Most studies have found an impairment of working memory, especially during "high-load" conditions. However, these studies have used either hydrocortisone administration (e.g., Lupien et al. 1999) or the TSST (e.g., Oei et al. 2006), which produce relatively high increases in cortisol concentrations. In contrast, studies using examination stress, which is associated with more moderate levels of increased cortisol concentrations have found both nonsignificant (Vedhara et al. 2000) and significant increases in working memory (Lewis et al. 2008). Together, the studies on stress and working memory support the inverted U relationship, with moderate levels of stress associated with improved working memory performance and high levels of stress associated with impairment in working memory.

Although the usage of examination stress studies increases the external validity of stress-related memory findings, a number of methodological limitations persist. For example, examination stress studies typically use successful college or postbaccalaureate students. Therefore, it is not surprising that these students perform well under typical examination stress conditions, especially examinations that do not result in high increases in cortisol concentrations. In order to truly test whether working memory conforms to the expected inverted $\mathrm{U}$ relationship as a function of level of stress, it is necessary to test subjects under different levels of an environmental stressor. To date, these studies have not been conducted. In addition, much more research is needed to better understand how examination stress affects different components of working memory, for example, the executive component versus the sensory component, and these tasks need to be tested under differing cognitive load conditions. Furthermore, we know very little regarding individual differences in working memory in response to examination stress. What are the characteristics and circumstances under which some perform well in response to examination stress and others do not? 
Whether one perceives the examination as a threat or a challenge, for example, is likely to result in differential working memory performance. Given the importance of examinations in our lives, and our dependency upon working memory during examinations, it is surprising that so little is known about the relationship between examination stress and working memory.

\section{Cross-References}

- Burnout in Teaching and Learning

- Coping with Stress

- Stress and Learning

- Stress Management

\section{References}

D’Esposito, M. (2007). From cognitive to neural models of working memory. Philosophical Transactions of the Royal Society B, 362, 761-772.

Lewis, R. S., Nikolova, A., Chang, D. J., \& Weekes, N. Y. (2008). Examination stress and components of working memory. Stress, $11,108-114$.

Lupien, S. J., Gillin, C., \& Hauger, R. L. (1999). Working memory is more sensitive than declarative memory to the acute effects of corticosteroids: a dose-response study. Behavioral Neuroscience, $113,420-430$.

Oei, N. Y. L., Everaerd, W. T. A. M., Elzinga, B. M., Van Well, S., \& Bermond, B. (2006). Social stress impairs working memory at high loads: An association with cortisol levels and memory retrieval. Stress, 9, 133-141.

Roozendaal, B. (2002). Stress and memory: Opposing effects of glucocorticoids on memory consolidation and memory retrieval. Neurobiology of Learning and Memory, 78, 578-595.

Vedhara, K., Hyde, J., Gilchrist, I. D., Tytherleigh, M., \& Plummer, S. (2000). Acute stress, memory, attention and cortisol. Psychoneuroendocrinology, 25, 535-549.

Weekes, N. Y., Lewis, R. S., Patel, F. R., Garrison-Jakel, J., Berger, D., \& Lupien, S. J. (2006). Examination stress as an ecological inducer of cortisol and psychological to stress in undergraduate students. Stress, 9, 199-206.

\section{Example-Based Learning}

\author{
AleXander RenkL \\ Department of Psychology, University of Freiburg, \\ Freiburg, Germany
}

\section{Synonyms}

Learning from worked examples; Learning from worked-out examples

\section{Definition}

One of the best established effects in research on learning and instruction is the worked example effect (for an overview see Renkl 2011). This effect refers to the robust finding that it is better for initial cognitive skill acquisition to study examples than to learn by problem solving.

Worked examples consist of a problem formulation and a solution. In many cases, solution steps are additionally provided, showing how to get to the solution. Such examples are commonplace in instructional materials pertaining to well-structured domains such as mathematics or physics. However, when proponents of this learning method discuss example-based learning, they typically mean that after the introduction of one or more domain principles (e.g., mathematical theorem, physics law) learners study several examples rather than a single example - as it is commonly the case. Despite this emphasis on examples, it is acknowledged that learners should solve problems later on in cognitive skill acquisition in order for them to reach proficiency in the respective domain.

\section{Theoretical Background}

Historically, the worked-example effect was found in research on cognitive load theory (Sweller and Cooper 1985). The traditional explanation provided by this theory is as follows. When problems are presented for cognitive skill acquisition, learners typically try to solve these problems by using general search strategies such as means-ends analyses. As a consequence, they focus on specific features of the problems at hand to reduce the difference between current problem states and the goal problem states rather than on schema-relevant principles. In addition, the task of reducing the difference between problem states requires learners to maintain subgoals and consider different solution options in working memory. These requirements can induce heavy cognitive load or even overload. Hence, performing general search strategies does not effectively contribute to the construction of problemsolving schemas that enable learners to detect relevant structural features in later problems to be solved and, on this basis, to select appropriate solution procedures. In a nutshell, problem solving imposes a substantial amount of unnecessary (extraneous) load that does not contribute to learning in the sense of schema construction. 
After the publication of the seminal studies that have established the worked-example effect (e.g., Sweller and Cooper 1985), research has led to a more differentiated account of this effect. One important extension of this early work are findings that the cognitive capacity freed up by presenting examples instead of problems is not productively used by all learners. Actually, many learners study worked examples in a passive or superficial way (see Renkl 2011). Hence, in order to really exploit the potential of example-based learning, learners have to be prompted or trained to process the examples more deeply. More specifically, learners should be enabled to self-explain the presented solution (steps) so that they can understand their underlying rationale. One very productive type of self-explanations are principle-based explanations (Renkl 2011), that is, learners explicate the domain principle (e.g., mathematical theorem) that is applied in a specific solution step (e.g., "Here the two probabilities are multiplied because the probability that both independent events come to happen should be determined"; in this case a learner explains a solution step by the "essence" of the multiplication rule in probability).

A second important extension refers to findings that the worked example effect disappears when the learners progress through the phases of cognitive skill acquisition. Finally, problem solving is more productive than studying examples when the learners have progressed to higher skill levels. The best option to structure the transition from studying examples to problem solving is to gradually fade the worked solutions. For example, after completely worked examples incomplete examples are presented so that the learners encounter first problem-solving demands. With time, the number of faded parts is increased step by step until the whole problem needs to be solved. It is best to fade those worked parts that are already understood by the learners - indicated by corresponding correct selfexplanations - because studying examples has then become a redundant activity that contributes little or nothing to further learning. In contrast, solving problems can then lead to the formation of correct automated mental rules (i.e., production rules).

A third significant extension is that example-based learning is meanwhile increasingly used not only in well-structured domains with mathematical solutions, as it is traditionally the case, but also for complex cognitive skills from ill-structured domains such as identifying designer styles, collaborating productively, engaging in scientific argumentation, or applying learning strategies in journal writing (for an overview see Renkl 2011). In some of these cases, video-based models are shown as examples so that the boundaries between example-based learning and observational learning research in the tradition of Bandura's sociocognitive learning theory get blurred (Renkl 2011).

Renkl (2011) has recently proposed a framework on example-based learning that integrates the above mentioned issues. In addition, this framework informs on important instructional design principles (e.g., eliciting self-explanations, fading) that help to fully exploit the potential of example-based learning.

\section{Important Scientific Research and Open Questions}

There is an abundance of studies on example-based learning so that a representative overview cannot be provided within this entry. As the "basics" of examplebased learning are meanwhile very well analyzed, the probably most interesting recent research has combined example-based learning with other tried-andtested instructional approaches. Three examples of such research are provided:

1. A way of implementing effective learning by problem solving was developed by the Cognitive Tutor approach (see www.carnegielearing.com). Cognitive Tutors are intelligent tutoring systems that continuously collect data on the learners in order to individually tailor the problems and hints presented for learning. These tutors have shown to effectively foster learning, in particular in mathematics. They are meanwhile used in many US schools. In a whole series of studies, it was found that "enriching" Cognitive Tutor with worked examples further improves this intelligent tutoring system (see Salden et al. 2010). The most robust effect of including examples is that substantial learning time is saved. In some cases, conceptual understanding is fostered as well. Overall, a fruitful synergy has been developed between examplebased learning and learning by intelligent tutoring.

2. Errors are often attributed an important role for meaningful learning. Classical worked examples do not contain errors. Recently, a number of studies analyzed the effects of studying and/or 
self-explaining incorrect examples, usually in addition to correct examples (e.g., Stark et al. 2011). Overall, the findings show that the combination of error-triggered learning and example-based learning can be effective. However, the demand to cope with such errors can overwhelm learners with low prior knowledge.

3. Confronting learners with multiple solutions is often considered as an important instructional ingredient for the development of flexible mathematics skills and deep understanding. Classical worked examples just present one solution. A number of studies have shown that examples presenting multiple solutions and guiding the learners to compare these solutions can lead to favorable learning outcomes (e.g., Rittle-Johnson et al. 2009). As in the case of examples with errors, however, considering multiple solutions can overwhelm learners with low prior knowledge.

Two important open issues for further research should be outlined. First, there are diverse research traditions relevant when considering example-based instruction. In addition to the already mentioned theory traditions (i.e., cognitive load theory and observational learning), research on analogical reasoning or on modeling in the sense of cognitive apprenticeship (Collins et al. 1989) are tightly connected with issues of learning from examples. An important challenge for further research is to come to integrative approaches so that not each research tradition has to "reinvent the wheel."

Second, a relatively comprehensive set of instructional guidelines (e.g., foster self-explanations, fading, including errors or multiple solutions) have been established for optimizing example-based learning (see Renkl 2011). However, the interrelations or interactions between the different guidelines are not well understood. In many cases, the guidelines cannot be combined in a straightforward way or such combinations do not lead to "additive," but interactive effects (see Renkl 2011). More theoretical and empirical analyses are necessary to understanding the interplay between the instructional guidelines in a principled way.

\section{Cross-References}

- Discovery Learning

- Guidance Fading Effect
Self-Explanation, Feedback, and the Development of Analogical Reasoning Skills

- Worked Example Effect

\section{References}

Collins, A., Brown, J. S., \& Newman, S. E. (1989). Cognitive apprenticeship: Teaching the crafts of reading, writing, and mathematics. In L. B. Resnick (Ed.), Knowing, learning, and instruction. Essays in honor of Robert Glaser (pp. 453-494). Hillsdale: Erlbaum.

Renkl, A. (2011). Instruction based on examples. In R. E. Mayer \& P. A. Alexander (Eds.), Handbook of research on learning and instruction (pp. 272-295). New York: Routledge.

Rittle-Johnson, B., Star, J. R., \& Durkin, K. (2009). The importance of prior knowledge when comparing examples: Influences on conceptual and procedural knowledge of equation solving. Journal of Educational Psychology, 101, 836-852.

Salden, R., Koedinger, K. R., Renkl, A., Aleven, V., \& McLaren, B. M. (2010). Accounting for beneficial effects of worked examples in tutored problem solving. Educational Psychology Review, 22, 379-392.

Stark, R., Kopp, V., \& Fischer, M. R. (2011). Case-based learning with worked examples in complex domains. Two experimental studies in undergraduate medical education. Learning and Instruction, 21, 22-33.

Sweller, J., \& Cooper, G. A. (1985). The use of worked examples as a substitute for problem solving in learning algebra. Cognition and Instruction, 2, 59-89.

\section{Excellence}

Expertise

\section{Executive Attention}

The current focus of working memory that is used in the service of decision making.

\section{Executive Control}

- Examination Stress and Components of Working Memory 


\section{Exemplar}

An exemplar is an item, object, or stimulus belonging to a category or categories.

\section{Exemplar Learning and Schematization in Language Development}

\author{
Kirsten AbBot-Smith ${ }^{1}$, Evan KidD ${ }^{2}$ \\ ${ }^{1}$ School of Psychology, University of Kent, \\ Canterbury, Kent, UK \\ ${ }^{2}$ School of Psychological Sciences, \\ University of Manchester, Manchester, UK
}

\section{Synonyms}

Abstraction; Exemplar-learning; Generalization; Instance-learning; Schematization

\section{Definition}

Schematization refers to the process of abstraction from experience of the key characteristics needed to understand or operate in a particular cognitive domain. In the domain of nonlinguistic categorization, one type of " schema" is the "prototype," which is a mental representation that contains the typical functional and perceptual features associated with a particular category (the "central tendency") (Rosch 1973). "Prototype" theory has traditionally been set against "exemplar-based" (or "instance" learning) models. Proponents of exemplar-learning theories posit that each particular encounter with an instance or exemplar of a particular category is retained in memory together with particular details of that encounter, which are not necessarily essential features of the category in question.

\section{Theoretical Background}

In language acquisition, schema- and exemplar-based approaches have been invoked to explain development at all levels of description (Bybee 2006). In this entry we briefly discuss these processes in phonological, lexical, morphological, and syntactic development. Regardless of the linguistic level of description, these approaches are united by the theoretical assumption that language is learnable given general-cognitive learning mechanisms that involve memorization and, in the case of exemplar approaches, generalization over stored instances. It is this sense that schema- and exemplarbased approaches to language development eschew claims that knowledge of language is largely innate, thus contrasting sharply with linguistic nativist or generative approach to language and learning (e.g., Pinker and Ullman 2002). Rather, the child is argued to come to the problem of language learning with a set of powerful general-purpose learning mechanisms but with little substantive prior knowledge (Tomasello 2003).

In the domain of child language development, theories which explicitly invoke exemplar-learning and/or schematization have tended to fall into one of two categories. In the first type of theory, exemplarlearning is argued to occur in the initial learning phrase, followed by gradual schematization (Tomasello 2003). In the second type of theory, exemplar models simulate prototype effects by, for example, retrieving training items in parallel and "summing" the similarity between the features (Bybee 2006).

In regards to phonology or the sound system of a language, linguistic nativist or generative approaches posit that children acquire the sound patterns of their language by deducing parameter settings or sets of constraints that categorize their input language given innate biases. In contrast, exemplar- and schema-based approaches to phonological development argue that children learn the phonological system using inductive processes, from the "ground up." In particular, exemplar- and schema-based approaches follow the functionalist dictum that knowledge is acquired through using (or attempting to use) language; that language cannot be separated from its intended communicative purpose. It is for this reason that proponents of exemplar-based phonology argue that the acquisition of phonology truly begins in earnest when children begin to produce meaningful words (or free morphemes), which are the basic units of language (e.g., Vihman and Croft 2007). In the exemplar-learning/ schematization view of phonological development, children's first word productions are exemplars that become templates that the child uses in order to target the production of new words beyond the child's existing range. Therefore, while the initial productions, 
which are the source of the template, are accurate, subsequent attempts at newly acquired words are less accurate because they often differ from the source. On this approach to phonological development, the child's knowledge of the inventory of sound patterns in their input language emerges on the basis of further word learning, which increases their repertoire of word templates. As such, the adult phonological system is argued to be derived from the process of acquired exemplars that acquire a schematic nature to them via abstraction.

Theories which emphasize exemplar-learning followed by subsequent schematization have also made inroads into theories of how children learn lexical semantics. However, in this area prototype theory (Rosch 1973) has also been very influential. Often the same empirical data can be interpreted from both viewpoints. To illustrate, Meints et al. (1999) found in looking-time studies of noun comprehension that 12-month-olds only looked longer at "typical" exemplars (e.g., "robin" for bird) when hearing the referent, whereas 18-month-olds allowed atypical exemplars to be referents of their "known" nouns. Similar results have been found for the comprehension of prepositions. A prototype account would be that 12- and 15-month-olds have only encountered typical exemplars of and thus their "category" or "schema" only consists of the key features of these typical exemplars. However, an exemplar-learning model could account for these findings if it were the case that parents only labeled typical exemplars of the objects and of "on" and "under" events.

Exemplar theories are also prevalent as accounts of morphological development. Morphology can be divided into inflectional, where the meaning of the word does not change but its grammatical status does (e.g., English progressive suffix+ing on the verb "walk") and derivational, in which a new word meaning is created (e.g., kind+ness). In inflectional morphology there is a debate as to whether children learn morphemes via a combination of symbolic "rules" and associative learning (e.g., Pinker and Ullman 2002), or whether all inflectional morphological paradigms can be learned by a single associative network in which generalization occurs through analogy to previously learned exemplars (Bybee 2006). Similarly, connectionist models of inflection acquisition are highly prevalent. The latter - like exemplar-models - are data-driven, but represent "exemplars" in a distributed fashion. In derivational morphology, generalization must occur through semantic or meaning-based - analogy and can most easily be accounted for by exemplar-learning with resultant schematization. Even for inflectional morphology there is evidence that adults will overgeneralize irregular past tense (e.g., producing "frunk" as the past of "frink") if the context provides them with semantic similarity to known verbs which take this pattern (e.g., "drinkdrunk"). There is also evidence that the "rule-like" behavior which children and adults show with regular inflections can be accounted for by type frequency, i.e., the number of lexical types which take that particular inflection (Bybee 2006; Tomasello 2003).

Syntax refers to the conventions for structuring sentences in a given language. In syntactic development there are two main approaches which explicitly invoke exemplar-learning. Firstly, there are various computational approaches, which can model frequency and syntactic priming (e.g., recency) effects which have been found to occur both in adult language processing and in child syntactic production. Secondly, there is the usage-based approach (Tomasello 2003), which claims that there are no linguistically specific syntactic categories but that the child gradually "schematizes" over learned exemplars in the input. Usage-based theorists have claimed that children's schemas for sentence frames, such as the transitive (i.e., a frame which requires both a subject and a direct object) are originally based around particular lexical items which frequently occur in transitive sentences in child-directed speech, such as frequent verbs, frequent proper nouns, or frequent pronouns and auxiliary verbs.

\section{Important Scientific Research and Open Questions}

There is a now large body of empirical evidence that children - and indeed adults - retain a great deal of idiosyncratic information pertaining to particular lexical semantic categories and syntactic categories and even phonemes. In their morpho-syntactic development, children do initially perform much better when a morpheme or syntactic category is presented together with a familiar lexical item (see Tomasello 2003). However, these lexical or "item-based" effects do not relate to actual sentence tokens but to lexically based schemas. Moreover, the actual mechanisms involved in usage-based schematization remain unclear. 
On the prototype model side of the debate, certainly for syntactic constructions such as the passive construction, there is evidence that adults and children appear to be accessing an underlying schema which has prototype properties, such as a central tendency and fuzzy boundaries. On the exemplar-learning side of the debate, there is also evidence for language-specific differences, which are input-related (e.g., the "passive" sentence structure is learned early in languages such as Inuktitut, in which passive sentences are quite frequent in the input). Another open question is how to interpret "exemplarmodel" computational simulations. In many simulations, "exemplars" are actually a syntactic parsing of the input strings in a format which is very close to certain generative approaches.

When applied to linguistic development, both prototype and exemplar-learning (and hybrid models) pose the problem of how "similarity" is defined. In the domain of phonology, similarity is usually defined on a perceptual level. In the domain of syntax, a definition of "similarity" involves function as well as form. This has led many linguistics and computer scientists, who have adopted exemplar-learning models of language, to argue that an exemplar is not identical to the "token" of that exemplar in the environment, but involves the classification or decoding of tokens in some fashion (Bybee 2006).

Finally, while both computational and usage-based theories of language development propose that exemplar-learning should occur prior to schematization and category-based productivity, this is not the only logical relationship. It is possible that categorybased induction occurs prior to exemplar (or exception)-learning or even that that category-based and exemplar-based induction relies on two distinct underlying neural systems.

\section{Cross-References}

- Analogical Models

- Connectionism

- Dynamic Modeling and Analogies

- Language Acquisition and Development

- Phonological Representation

- Psycholinguistics and Learning

- Schemas

- Speech Perception and Learning

- Word Learning

\section{References}

Bybee, J. (2006). From usage to grammar: The mind's response to repetition. Language, 82(4), 711-733.

Meints, K., Plunkett, K., \& Harris, P. (1999). When does an ostrich become a bird? The role of typicality in early word comprehension. Developmental Psychology, 35(4), 1072-1078.

Pinker, S., \& Ullman, M. (2002). The past and the future of the past tense. Trends in Cognitive Sciences, 6(11), 456.

Rosch, E. (1973). Natural categories. Cognitive Psychology, 4, 328-350.

Tomasello, M. (2003). Constructing a language: A usage-based theory of language acquisition. Cambridge, MA: Harvard University Press.

Vihman, M. M., \& Croft, W. (2007). Phonological development: Toward a 'radical' templatic phonology. Linguistics, 45(100), 683-725.

\section{Exemplar-Learning}

- Exemplar Learning and Schematization in Language Development

\section{Exertion of Learning}

- Learning Activity

\section{Existential Perspective on Burnout}

Burnout in Teaching and Learning

\section{Expansive Learning}

- Contradictions in Expansive Learning

\section{Expansive Learning and Its Conditio Sine Qua Non}

\footnotetext{
- Contradictions in Expansive Learning
} 


\section{Expectancy Learning and Evaluative Learning}

\author{
Dirk Hermans, Frank Baeyens
}

Center for the Psychology of Learning and

Experimental Psychopathology, Department of

Psychology, University of Leuven, Leuven, Belgium

\section{Synonyms}

Expectancy learning and referential learning

\section{Definition}

A classical conditioning procedure typically consists of the contingent presentation of two stimuli. In most cases, one stimulus is neutral (e.g., tone; conditional stimulus, CS), whereas the second has a more pronounced meaning (e.g., shock, food; unconditional stimulus, US). As a result of this procedure of CS-US pairings, changes in responding to the CS can be observed. For instance, in a typical differential fear conditioning preparation, one neutral tone (CS+; e.g., $300 \mathrm{~Hz}$ ) is followed by the contingent presentation of an electric shock (US), while a second neutral tone (CS-; e.g., 1,000 Hz) is never followed by the US. Changed responding to the $\mathrm{CS}+$ could include increased skin conductance responses, higher scores on a fear rating scale, and the avoidance of the CS+ in a behavioral test. In this example, the contingent presentation of CS+ and US, and the unreinforced presentations of the CS- are the classical conditioning "procedure." The changes in responses that follow from this procedure are the "result" of the classical conditioning procedure. Both procedure and result have to be distinguished from the conditioning "process." This latter concept refers to the psychological/biological mechanism(s) that are assumed to be responsible for the conditioning results. The process is the pathway through which the procedure impacts changes in responses. In contrast with the procedure and the conditioning results, the conditioning process cannot be directly observed. The distinction between these three layers is important for maintaining theoretical clarity, in particular for a correct understanding of the difference between expectancy learning (or signal learning) and evaluative learning.
With respect to the "results" of conditioning, different types of outcome have been observed. For instance, in the above mentioned example of an originally neutral tone that is contingently followed by an aversive shock, one can observe that after several trials the presentation of the CS+ will elicit fear as well as the active expectancy that the US will be presented. In addition, however, one can observe that the CS+ acquires a more negative valence; i.e., the CS+ is rated as more "negative" or "disliked." In other words, as a result of the conditioning procedure, the CS+ will actively elicit the expectancy of the US, and at the same time is endowed with a more negative evaluative meaning (Hermans et al. 2002). These two types of outcome are referred to as expectancy learning and evaluative learning.

\section{Theoretical Background}

There are reasons to assume that evaluative conditioning and expectancy learning not only refer to a different outcome, but might also be based on different types of processes. This possibility is inferred from the observation that both outcomes respond differently to certain procedural changes.

One of these procedural changes pertains the statistical contingency between CS and US. If they are always presented together and never presented alone, there is a perfect contingency between both stimuli. One can, however, create procedural deviations from this situation; for instance by inserting trials in which the CS is presented without being followed by the US (CS-only trials), or by inserting trials on which the US is not preceded by the CS (US-only trials). It is obvious that such departures from a perfect contingency impact the US expectancy. The participant will experience that the CS is not a perfect predictor of the US. In certain cases, the CS can completely lose its objective predictive power, e.g., when the probability of the US in the presence of the CS equals the probability of the US in the absence of the CS, [i.e., $P(\mathrm{USICS})=P(\mathrm{USIn}$ - $\mathrm{CS})$ ] It is known from many studies, including the seminal work by Rescorla (1968), that the statistical contingency between the CS and the US has a crucial impact on the amount of (fear) conditioning. Descriptively, one could argue that one process that drives classical conditioning is signal learning. Confronted with a significant events like the presentation of the US (e.g., shock), the organism will look for stimuli (CSs) 
that are valid predictors. This results in the CS acquiring the capacity to generate an active expectancy of a US-going-to-occur-here-and-now. It quite naturally follows from this that CS-US contingency is a prerequisite for signal learning and conditioned preparatory responding.

For evaluative learning, however, there are strong indications that deviations from a perfect contingency are less or not relevant for the amount of learning. In a study by Baeyens et al. (1993), for instance, it was observed that manipulating the level of CS-US contingency did not significantly impact evaluative learning. This was confirmed by a recent meta-analysis of over 200 evaluative conditioning (EC) studies, which showed that the number of CS-only and US-only trials during the acquisition phase had no significant effect on the evaluative conditioning effect sizes (Hofmann et al. 2010). This led the authors to conclude that: “... the degree of statistical contingency between the CS and the US during acquisition has little, if any, effect on the magnitude of EC. The fact that other forms of Pavlovian conditioning strongly depend on the statistical contingency between the CS and the US, ..., suggests that EC is unique in this respect" (Hofmann et al. 2010, p. 22).

A second example of how procedural variations can differentially impact changes in US-expectancy and in evaluative responses is extinction. Pavlovian extinction refers to a procedure in which, after extensive acquisition, the organism is confronted with repeated CS-only presentations. In a typical experiment, the first phase (acquisition phase) would consist of repeated contingent presentations of a neutral CS and a shock US. As a result, the CS will elicit the active expectation of the US as well as increased fear responses. In a second phase (extinction phase), the CS is then repeatedly presented without being followed by the US. As a result of these unreinforced CS presentations, one can observe marked decreases in conditioned responses. This observation has clear clinical relevance: fear reduction as a result of CS-only trials provides a good laboratory analogue for exposure treatment of anxiety disorders. The impact of extinction can be easily understood from a signal-learning perspective: the organism actually learns that the CS is no longer a valid predictor for the US. As a result, it no longer expects the US upon confrontation with the CS, and fear for this stimulus will decline and disappear.
Interestingly, several studies have demonstrated that evaluative learning is less impacted by an extinction procedure. However, whereas initial studies suggested a complete "resistance to extinction," the aforementioned meta-analysis revealed that this is overstated in that overall, extinction treatment resulted in reduced but still highly reliable evaluative responding. Also, there are strong indications that evaluative conditioning is "less sensitive" to extinction than expectancy learning. A good example is a study by Vansteenwegen et al. (2006) in which a fear conditioning preparation was used. Participants were presented with two pictures of a human face, one of which was contingently followed by an electrocutaneous stimulus $(\mathrm{CS}+)$, while the other was not (CS-). After eight acquisition trials, a lengthy extinction phase followed which consisted of 24 unreinforced presentations of both CSs. Evaluative changes as a result of acquisition and extinction were assessed by means of a reaction time procedure (affective priming task) that was scheduled immediately after acquisition and after extinction. As a measure of US expectancy, skin conductance responses were obtained throughout the experiment. The authors observed that whereas fast extinction was obtained for expectancy learning, as measured by the SCR, the affective priming task clearly showed resistance to extinction of evaluative learning. Because a differentiation between the two types of learning was demonstrated in one and the same paradigm using an extended extinction procedure, this study can be considered as strong evidence for a limited sensitivity to extinction of evaluative conditioning (Vansteenwegen et al. 2006, p. 75). This differential sensitivity to extinction has clinical as well as theoretical implications. Clinically, it means that if exposure has taken away all fear for the phobic object, the patient might still experience it as a negative stimulus. This stimulus valence might entail risks for return of fear. Theoretically, it further sustains the possibility that evaluative learning and signal learning (defined as outcomes) are based on qualitatively different processes.

How then should one conceptualize this difference between evaluative learning and expectancy learning at a process level? One possible view on this issue makes a distinction between the acquisition of propositional versus of less-than-propositional (or "merely referential") knowledge (Baeyens et al. 2001). The first relates 
to the observation that the organism learns to expect the US upon presentation of the CS. It learns to believe/ expect that "the US will appear soon." The CS has become a valid signal for the US, which is mentally represented by the fact that it elicits the proposition "This stimulus predicts the US" or "The US will appear soon." This proposition can be true or false, and new experiences can sustain or contradict this proposition. This explains why US-expectancy responses are influenced by changes in the statistical contingency between the CS and US, as well as by extinction trials: these events entail evidence against or for the (to be) learned proposition. In extinction, for example, the organism gradually learns that this proposition is no longer true. This is reflected in changes in US expectancy (and the fear that is derived from this).

In evaluative learning, it is assumed that a "lessthan-propositional" mental state is elicited by the CS. This mental state could best be captured as "merely referential." The CS makes one think of the US, or "refers" to the US without eliciting an active expectation or prediction of its occurrence. One has learned an association between the CS and the US, and activation of the memory representation of the CS will activate the representation of the US. The CS evokes the thought of the US. Evaluative learning is assumed to be an instance of such merely referential learning. The $\mathrm{CS}$ acquires a positive (or negative) meaning through its association with the negative (or positive) US. Note that this type of non-propositional knowledge is inherently immune to changes in CS-US contingency and to procedures of extinction. For instance, if one has frequently encountered a certain song in the context of a pleasant holiday, this association might lead to a change in liking for that particular song. When one encounters the song later on, in another context, it might engender reminiscences about the vacation. Repeatedly hearing the song, however, will not necessarily extinguish these memories. In contrast with "active expectations," which can be "true" or "false," mere "reference to" cannot be refuted or supported by occurrence or nonoccurrence of the US.

\section{Important Scientific Research and Open Questions}

The differentiation between expectancy learning (signal learning) on the one hand and referential learning on the other is theoretically appealing. The study of evaluative learning fits almost naturally within the conceptualization of learning as a referential process. It will be obvious, however, that unlike the observation of evaluative learning as a "result," which is a factual and replicable event, the "referential process" is a hypothetical construct. It is a theoretical view that provides an explanation for observations such as the differential sensitivity to variations in CS-US contingency as well as to procedures of extinction. The validity of the referential account has been a matter of discussion (e.g., Hofmann et al. 2010). In particular, propositional accounts have been developed to explain evaluative changes that are acquired through conditioning. Future research will be necessary to assess the validity of the referential versus the propositional model.

\section{Cross-References}

- Affective Priming and Learning

- Conditioning and Anxiety

- Evaluative Conditioning

- Fear Conditioning in Animals and Humans

- Pavlovian Conditioning

\section{References}

Baeyens, F., Hermans, D., \& Eelen, P. (1993). The role of CS-US contingency in human evaluative conditioning. Behaviour Research and Therapy, 31, 731-737.

Baeyens, F., Vansteenwegen, D., Hermans, D., \& Eelen, P. (2001). Chilled white wine, when all of a sudden the doorbell rings: Mere reference and evaluation versus expectancy and preparation in human Pavlovian learning. In F. Columbus (Ed.), Advances in Psychology Research (Vol. 4, pp. 241-277). Huntington: Nova Science.

Hermans, D., Vansteenwegen, D., Crombez, G., Baeyens, F., \& Eelen, P. (2002). Expectancy-learning and evaluative learning in human classical conditioning: Affective priming as an indirect and unobtrusive measure of conditioned stimulus valence. Behaviour Research and Therapy, 40, 217-234.

Hofmann, W., De Houwer, J., Perugini, M., Baeyens, F., \& Crombez, G. (2010). Evaluative conditioning in humans: A meta-analysis. Psychological Bulletin, 136, 390-421.

Rescorla, R. A. (1968). Probability of shock in the presence and absence of CS in fear conditioning. Journal of Comparative and Physiological Psychology, 66, 1-5.

Vansteenwegen, D., Francken, G., Vervliet, B., Declerq, A., \& Eelen, P. (2006). Resistance to extinction in evaluative conditioning. Journal of Experimental Psychology: Animal Behavior Processes, $32,71-79$. 


\section{Expectancy Learning and Referential Learning}

Expectancy Learning and Evaluative Learning

\section{References}

Rogers, C. (1959). A theory of therapy, personality and interpersonal relationships as developed in the client-centered framework. In S. Koch (Ed.), Psychology: A study of a science. Vol. 3: Formulations of the person and the social context. New York: McGraw Hill.

\section{Expectancy-Value Theory}

Motivation is seen as a result of expecting and valuing success in an activity.

\section{Expectation}

Many Aspects of Anticipation

\section{Expectation-Based Behavior}

Surprise and Anticipation in Learning

\section{Experience}

- Expertise

- Inhibition and Learning

\section{Experience (noun)}

The term experience is used to include all that is going on within the envelope of the organism at any given moment and which is available to awareness. It includes events of which the individual is unaware, as well as all the phenomena that are in consciousness. Experience refers to the given moment, not to some accumulation of past experience (Rogers 1959).

\section{Experience (verb)}

To experience means simply to receive in the organism the impact of the sensory of physiological events that are happening at the moment (Rogers 1959).

\section{References}

Rogers, C. (1959). A theory of therapy, personality and interpersonal relationships as developed in the client-centered framework. In S. Koch (Ed.), Psychology: A Study of a science. Vol. 3: Formulations of the person and the social context. New York: McGraw Hill.

\section{Experience Based Learning}

\section{Experiential Learning Theory}

\section{Experience-Based Reasoning}

\section{- Introspective Learning to Build Case-Based} Reasoning

\section{Experience-weighted Attraction Learning}

Experience-weighted attraction (EWA) learning is a theoretical term within the realm of machine learning and includes reinforcement learning and a set of weighted fictitious play belief models as special cases. In EWA, strategies have attractions which reflect prior predispositions, are updated based on payoff experience, and determine choice probabilities according to some rule (e.g., logit). 


\section{Experiencing Wisdom Across the Lifespan}

\author{
Sarah E. Ainsworth ${ }^{1}$, Susan Bluck ${ }^{2}$, Judith GlüCK ${ }^{3}$ \\ ${ }^{1}$ Department of Psychology, Florida State University, \\ Tallahassee, FL, USA \\ ${ }^{2}$ Department of Psychology, University of Florida, \\ Gainesville, FL, USA \\ ${ }^{3}$ Institut für Psychologie, Alpen-Adria Universität \\ Klagenfurt, Klagenfurt, Austria
}

\section{Synonyms}

Sagacity; Sapience

\section{Definition}

Wisdom is considered a relatively rare human virtue. An exact definition has eluded philosophers and scholars for centuries. The word wisdom in old English, Greek, and German languages can be traced back to the Indo-European word, wede, which means "to see" or "to know." The definition of wisdom has changed over history and varies across cultures, but certain central features including the original aspects (insight and knowledge) have remained consistent. Paul Baltes and colleagues provided a definition of wisdom in the psychological literature as entailing expertise in the fundamental pragmatics of life. Monika Ardelt's definition of wisdom has also been influential. She argues that wisdom involves the integration of three broad components: affect (i.e., compassion), cognition, and reflection. Her definition highlights that compassion or concern for the common good may be central to wisdom. More recently, Ursula Staudinger posited that definitions of wisdom in the literature can be classified as referring to either personal wisdom, related to insights about one's own self and life, or general wisdom about life. Debate exists as to whether wisdom is best conceptualized as a mature form of personality development or as a type of knowledge-based expertise. Regardless, most researchers agree that wisdom does not solely involve theoretical considerations but must manifest itself practically in response to fundamental and challenging life problems. Wisdom has historically been associated with older persons, (i.e., the adage that "older is wiser"), but current conceptions of wisdom suggest that, though wisdom may depend on rich and varied experience with life, old age is neither a necessary nor a sufficient condition for its development.

\section{Theoretical Background}

Writings on wisdom date back 5,000 years to the Sumerians in Mesopotamia. The modern conception of wisdom as a virtue began with Greek philosophers, including Socrates, Plato, and Aristotle. The history of wisdom is rife with philosophical and religious associations, and current research continues to probe the relation between wisdom and religion or spirituality, as well as the relation of wisdom to satisfaction with one's life. For many years, social scientists considered wisdom an unsuitable topic for scientific study because it could not be easily defined or measured and is valueladen. Pioneering work by Paul Baltes and colleagues in the 1980s helped to foster research on wisdom within psychology by developing systematic theory and methods (i.e., The Berlin Wisdom Paradigm). Although the field remains relatively small, many researchers have made valuable contributions to the theoretical understanding of wisdom since the 1980s, including Carolyn Aldwin, Monika Ardelt, Jim Birren, Deirdre Kramer, Ute Kunzmann, Rick Levenson, Willis Overton, Jacqui Smith, Ursula Staudinger, Bob Sternberg, Masami Takahashi, Jeff Webster, Paul Wink, ourselves, and others.

Theoretical and empirical research in this area is often categorized as concerning either implicit or explicit wisdom. This distinction, originally employed in Bob Sternberg's intelligence research, allows investigators to distinguish laypersons' implicit views of what wisdom entails from expert's theoretical conceptions of wisdom. Research on implicit theories of wisdom documents how laypersons conceptualize and mentally represent wisdom. It examines what people perceive wisdom to be, who they consider wise, and more recently, how they believe wisdom develops. Researchers have shown that implicit theories of wisdom vary within and between cultures (e.g., Eastern and Western conceptions of wisdom), and across people of different age and gender. Explicit theories of wisdom are accounts of wisdom formulated by scholars. Such theories are based on philosophical writings, psychological constructs and empirical research often related to lifespan developmental processes. A major goal of explicit theories of wisdom is to provide a framework for the continued design of research 
and development of instruments or procedures for assessing a person's level of wisdom.

\section{Important Scientific Research and Open Questions}

The majority of research on implicit theories of wisdom tests whether people's conceptions of what wisdom entails are related to factors such as their place in the lifespan, gender, or cultural background. Common methodological approaches include having participants rate a broad series of adjectives or phrases for their similarity to wisdom, nominate public figures or personally-known people they perceive to be wise, assess the level of wisdom of target people using experimental manipulations (e.g., target is male versus female), and write autobiographical narratives about a time they personally felt they did something wise. Bluck and Glück's review of the literature (2005) shows that five components emerge as central to laypersons' implicit theories of wisdom: high cognitive ability, insight into life problems, a reflective attitude, compassion for others, and practical skills to manifest one's wisdom in life. Note that these components of implicit wisdom map quite well onto experts' explicit theories of wisdom.

Layperson conceptions of wisdom have been shown to vary by age and gender. For example, men report wisdom events from different domains than women, and, as people get older, they are less likely to believe that older persons are wiser. Whereas younger people often view knowledge and insight as critical components of wisdom, across adulthood people increasingly develop a more integrative view of wisdom in which empathy, tolerance, or love for humanity are also viewed as necessary components. Although wisdom is generally associated with age, research on implicit theories of wisdom indicates that laypersons actually see age as correlated with, but neither necessary nor sufficient for the development of wisdom (Bluck and Glück 2005). To explore layperson's implicit theories of wisdom, researchers have also assessed the different forms that wisdom can take. People of different age groups who wrote about a time they thought, did, or said something wise in their own life reported different forms of wisdom (Glück et al. 2005). Adolescents most often wrote about a time they showed empathy and support, people in midlife most often wrote about an incident involving self-determination and assertion, and older people most often wrote about a time they exhibited knowledge and flexibility. A new area in implicit wisdom research is the investigation of people's implicit theories of the ontogenesis of wisdom. That is, how do people think that a person becomes wise? The first research in this area suggests that people believe that having a broad range of positive and negative experiences and the opportunity to learn from wise people are critical in the development of wisdom, whereas age itself is less important. The cultural association of wisdom exclusively with old age likely reflects the role of life experience. Experience with challenging life events is viewed as central to the development of wisdom, and older people are assumed to have had more of these experiences.

Research on explicit wisdom presents researchers with the challenge of finding an objective method to measure actual wisdom. The Berlin Wisdom Paradigm measures individual levels of wisdom by having participants provide open-ended responses to scenarios describing fundamental life issues (Baltes and Smith 1990). For example, participants respond to a hypothetical scenario, such as "Imagine that someone gets a call from a good friend who says that he or she cannot go on anymore and wants to commit suicide. What could that person consider and do?" Participants' answers are content coded for adherence to five theoretically defined criteria: factual knowledge about human development and human nature, procedural knowledge concerning how to deal with life issues, recognition of the lifespan contextual nature of events, acceptance of the relativity of human values, and tolerance for uncertainty. Performance in the Berlin Wisdom Paradigm, usually labeled wisdom-related knowledge, increases from about age 14 to 25 and is then stable across adulthood (Baltes and Staudinger 2000). As such, people in adolescence and early adulthood do not show the same capacity for wisdomrelated knowledge as do people in early midlife and beyond. Note however, that across several studies, wisdom has been found to be stable and does not continue to increase with chronological age across adulthood. Wisdom-related knowledge is correlated with intelligence and openness to experience as well as to measures located at the interface of cognition and personality, such as creativity, moral reasoning, and cognitive style. A new method has recently been developed by Ursula Staudinger and colleagues to assess personal wisdom. This approach uses open-ended questions about situations in the participants' own life. 
Researchers have also measured wisdom using selfreport scalar measures. Monika Ardelt's threedimensional wisdom scale (3D-WS) measures wisdom as a personality trait with cognitive, reflective, and affective components. Scores in the 3D-WS are related to mastery, subjective well-being, purpose in life, and subjective health, and negatively related with depressive symptoms, death avoidance, fear of death, and feelings of economic pressure (Ardelt 2003). The self-assessed wisdom scale (SAWS; Jeffrey D. Webster) measures wisdom directly by assessing experience, openness, emotion regulation, reminiscence, and humor. SAWS scores are positively correlated to generativity and ego integrity, and negatively to attachment avoidance. A scale developed by Rick Levenson and Carolyn Aldwin, the Adult Self-Transcendence Inventory (ASTI), measures wisdom as self-transcendence.

Given that it is clear that wisdom does not come naturally with age, theorists have proposed potential mechanisms for the development of explicit wisdom. Most have yet to be empirically tested. Researchers have posited that expertise-related factors (e.g., motivation to learn about life or experience with challenges) or facilitative contexts (e.g., educational opportunities) may contribute to the development of wisdom (Baltes and Staudinger 2000). Glück and Bluck are currently proposing a theory suggesting that four critical personal resources are necessary for the development of wisdom and that these are brought to bear as one encounters life events across the lifespan: mastery, openness to experience, being reflective about life events, and having good emotion regulation skills. Charting the factors that increase the likelihood that one will become wise across the lifespan is a clear direction for future research. Other open questions include whether wisdom can be taught and whether certain social and institutional environments promote or discourage the development and expression of wisdom across the lifespan.

\section{Cross-References}

- Adaptation and Anticipation: Learning from Experience

- Change of Values Through Learning

- Cognitive Aging

- Development of Expertise

- Post-traumatic Growth

- Values and Lifelong Learning

\section{References}

Ardelt, M. (2003). Empirical assessment of the three-dimensional wisdom scale. Research on Aging, 25, 275-324.

Baltes, P. B., \& Smith, J. (1990). Toward a psychology of wisdom and its ontogenesis. In R. Sternberg (Ed.), Wisdom: In nature, origins, and development (pp. 87-120). Cambridge, MA: Cambridge University Press.

Baltes, P. B., \& Staudinger, U. M. (2000). Wisdom: A metaheuristic (pragmatic) to orchestrate mind and virtue toward excellence. The American Psychologist, 55, 122-136.

Bluck, S., \& Glück, J. (2005). From the inside out: People's implicit theories of wisdom. In R. Sternberg \& J. Jordan (Eds.), A handbook of wisdom: Psychological perspectives (pp. 84-109). New York: Cambridge University Press.

Glück, J., Bluck, S., Baron, J., \& McAdams, D. (2005). The wisdom of experience: Autobiographical narratives across adulthood. International Journal of Behavioral Development, 29, 197-208.

\section{Experiential Education}

\author{
Laboratory Learning
}

\section{Experiential Learning}

- Action-Based Learning

- Adult Teaching and Learning

- Episodic Learning

- Experiential Learning Theory

- Incidental Learning

- Informal Learning

- Learning by Doing

- Phenomenology of Learning

\section{Experiential Learning Spaces}

\author{
Alice Y. Kolb, David A. Kolb
}

Organization Behavior Department, Weatherhead

School of Management, Case Western Reserve

University, Cleveland Heights, OH, USA

\section{Synonyms}

Learning contexts; Learning environments 


\section{Definition}

In experiential learning theory (ELT) (Kolb 1984) the concept of learning space, based on Kurt Lewin's field theory and his concept of life space, is used to elaborate the complex and dynamic nature of learning style and its formation through transactions between a person and his/her environment. This space exists in the experience of a learner and is formed both by objective factors such as the physical setting and time available for learning and by subjective factors such as learning preferences and expectations. The Kolb Learning Style Inventory (KLSI) (Kolb and Kolb 2005a) measures an individual's preference for a particular region of the learning space, their home region so to speak.

\section{Theoretical Background}

To elaborate further the complex, dynamic nature of learning style and its formation through transactions between the person and environment ELT uses the concept of learning space. The concept of learning space builds on Kurt Lewin's field theory and his concept of life space. For Lewin, both person and environment are interdependent variables, a concept Lewin translated into a mathematical formula, $B=f(p, e)$ where behavior is a function of person and environment. The life space is the total psychological environment which a person experiences subjectively. Life space includes all facts which have existence for a person and excludes those which do not. It embraces needs, goals, unconscious influences, memories, beliefs, events of a political, economic, and social nature, and anything else that might have an effect on behavior. The various factors in a given life space are to some degree interdependent, and Lewin strongly maintains that only the dynamic concepts of tension and force can deal with these sets of interdependent facts. This is what led him to define psychological needs as tension systems and their topological representation as vectors to denote motion. He postulates that the particular organization of a person's life space is determined by a field of forces, both internal needs and external demands, that positions the individual in a life space composed of different regions. Using a map-like representation the life space could be depicted topologically. Life spaces can vary in a number of dimensions including extension, differentiation, integration, and level of conflict. Lewin introduced a number of concepts for analysis of the life space and a person's relationship to it that are applicable to the study of learning spaces, including position, region, locomotion, equilibrium of forces, positive and negative valence, barriers in the person and the world, conflict, and goal.

Three other theoretical frameworks inform the ELT concept of learning space. Urie Bronfrenbrenner's (1977) work on the ecology of human development has made significant sociological contributions to Lewin's life-space concept. He defines the ecology of learning/development spaces as a topologically nested arrangement of structures each contained within the next. A learner's immediate setting such as a course or classroom is called a microsystem, while other concurrent settings in a person's life such as other courses, the dormitory, or family are referred to as the mesosystem. An exosystem encompasses the formal and informal social structures that influence a person's immediate environment, such as institutional policies and procedures and campus culture. Finally, the term macrosystem is used to refer to the overarching institutional patterns and values of the wider culture, such as cultural values favoring abstract knowledge over practical knowledge, that influence actors in a person's immediate microsystem and mesosystem. This theory provides a framework for analysis of the social system factors that influence learners' experience of their learning spaces.

Another important contribution to the learning space concept is situated learning theory (Lave and Wenger 1991). Like ELT situated learning theory draws on Vygotsky's activity theory of social cognition for a conception of social knowledge that conceives of learning as a transaction between a person and a social environment. Situations in situated learning theory like life space and learning space are not necessarily physical places but constructs of a person's experience in a social environment. These situations are embedded in communities of practice that have a history, norms, tools, and traditions of practice. Knowledge resides not in an individual's head, but in communities of practice. Learning is thus a process of becoming a member of a community of practice through legitimate peripheral participation (e.g., apprenticeship). Situated learning theory enriches the learning space concept by highlighting that learning spaces extend beyond a teacher and a classroom. They include socialization into a wider community of practice that involves 
membership, identity formation, transitioning from novice to expert through mentorship and experience in the activities of the practice, as well as the reproduction and development of the community of practice itself as newcomers replace old-timers.

Finally, in his theory of knowledge creation Nonaka introduces the Japanese concept of "ba," a "context that harbors meaning," which is a shared space that is the foundation for knowledge creation. "Knowledge is embedded in $b a$, where it is then acquired through one's own experience or reflections on the experiences of others" (Nonaka and Konno 1998, p. 40). Knowledge embedded in $b a$ is tacit and can only be made explicit through sharing of feelings, thoughts, and experiences of persons in the space. For this to happen the $b a$ space requires that individuals remove barriers between one another in a climate that emphasizes "care, love, trust, and commitment." Learning spaces similarly require norms of psychological safety, serious purpose, and respect to promote learning.

The ELT learning space concept emphasizes that learning is not one universal process but a map of learning territories, a frame of reference within which many different ways of learning can flourish and interrelate. It is a holistic framework that orients the many different ways of learning to one another. In ELT an experiential learning space is defined by the attracting and repelling forces (positive and negative valences) of the two poles of the dual dialectics of action/reflection and experiencing/conceptualizing, creating a twodimensional map of the regions of the learning space. An individual's learning style positions them in one of these regions depending on the equilibrium of forces among action, reflection, experiencing, and conceptualizing. As with the concept of life space, this position is determined by a combination of individual disposition and characteristics of the learning environment. One's position in the learning space defines their experience of it and it is this experience that defines their "reality." The KLSI measures an individual's preference for a particular region of the learning space, their home region so to speak. Learners' scores on the KLSI place them in one of the nine regions depicted in Fig. 1, each of which is associated with a specific learning style and process of learning from experience. These regions are named using the points of a compass and are divided into specialized learning regions and integrative or balancing learning regions. The regions of the ELT

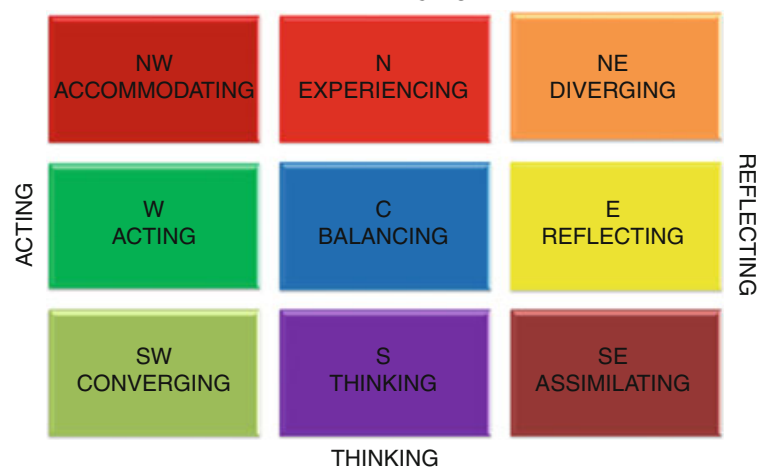

Experiential Learning Spaces. Fig. 1 The nine regions of the experiential learning space

learning space offer a typology of the different types of learning based on the extent to which they require action vs. reflection, experiencing vs. thinking, thereby emphasizing some stages of the learning cycle over others.

The learning process in specialized learning regions, NW, NE, SE, and SW, strongly emphasizes one pole of the experiencing/thinking dialectic and one pole of the acting/reflecting dialectic. Individuals in the NW region learn primarily through acting and experiencing. In the NE region learners emphasize reflecting and experiencing. In the SE region learners emphasize reflecting and thinking. In the SW region individuals learn through thinking and acting.

In the integrative learning regions, $\mathrm{N}, \mathrm{E}, \mathrm{S}, \mathrm{W}$, and $\mathrm{C}$, the learning process integrates the poles of one or both of the two dialectics. The learning process in the $\mathrm{N}$ region integrates acting and reflecting with a primary emphasis on feeling. In the $\mathrm{E}$ region the learning process integrates feeling and thinking with a primary emphasis on reflecting. In the $S$ region learners integrate acting and reflecting with a primary emphasis on thinking. In the $\mathrm{W}$ region the learning process integrates feeling and thinking with a primary emphasis on action. In the Central region learners take an integrative approach to learning that balances experiencing, thinking, acting, and reflecting.

\section{Important Scientific Research and Open Questions}

Recent research has used the KLSI to assess institutional learning spaces using the aggregates of human characteristics approach defined by Strange and 
Banning (2001), who argue in Educating by Design that the psychological and social dimensions of learning spaces have the most influence on learning (Kolb and Kolb 2005b).

Other research is focusing on the enhancement of experiential learning in higher education and the creation of learning spaces that promote growth-producing experiences for learners. In our recent research on experiential learning, we have focused on the importance of learning spaces and have developed principles for creating them (Kolb and Kolb 2005b). For a learner to engage fully in the learning cycle, a space must be provided to engage fully in the four modes of the cycle - feeling, reflection, thinking, and action. It needs to be a hospitable, welcoming space that is characterized by respect for all. It needs to be safe and supportive, but also challenging. It must allow learners to be in charge of their own learning and allow time for the repetitive practice that develops expertise. Other work is focusing on the process of experiential learning viewed as a process of locomotion through the learning regions that is influenced by a person's position in the learning space. Research using the Learning Flexibility Index (Sharma and Kolb 2010) has shown that individuals vary in their ability to move about the learning space from their home region and that this capacity to adapt flexibly to changing learning contexts is related to higher stages of adult development (Kolb 1984, Chap. 8).

\section{Cross-References}

- Experiential Learning Cycle

- Experiential Learning Spiral

- Experiential Learning Theory

- Learning Identity

- Learning Style

\section{References}

Bronfrenbrenner, U. (1977). Toward an experimental ecology of human development. The American Psychologist, 32(7), 513-530.

Kolb, D. A. (1984). Experiential learning: Experience as the source of learning and development. Englewood Cliffs: Prentice-Hall.

Kolb, A. Y., \& Kolb, D. A. (2005a). The Kolb learning style inventoryversion 3.1: 2005 technical specifications. Boston: Hay Transforming Learning. www.haygroup.com/tl

Kolb, A. Y., \& Kolb, D. A. (2005b). Learning styles and learning spaces: Enhancing experiential learning in higher education. Academy of Management Learning and Education, 42(3), 193-212.
Lave, J., \& Wenger, E. (1991). Situated learning: Legitimate peripheral participation. Cambridge: Cambridge University Press.

Nonaka, I., \& Konno, N. (1998). The concept of "ba": Building a foundation for knowledge creation. California Management Review, 40(3), 40-54.

Sharma, G., \& Kolb, D. A. (2010). The learning flexibility index: Assessing contextual flexibility in learning style. In S. Rayner \& E. Cools (Eds.), Style differences in cognition, learning and management: Theory, research and practice. New York: Routledge.

Strange, C. C., \& Banning, J. H. (2001). Educating by design: Creating campus learning environments that work. San Francisco: Jossey-Bass.

\section{Experiential Learning Spiral}

\author{
Alice Y. Kolb, David A. Kolb
}

Organization Behavior Department, Weatherhead

School of Management, Case Western Reserve

University, Cleveland, $\mathrm{OH}$, USA

\section{Synonyms}

Recursive learning cycle

\section{Definition}

The experiential learning spiral describes the ongoing recursive operation of the experiential learning cycle of experiencing, reflecting, thinking, and acting. The cycle is not a circle but a spiral since each trip through the cycle returns to the experience with new insight gained by reflection, thought, and action. Thus, the experiential learning spiral describes how learning from experience leads to development.

\section{Theoretical Background}

In experiential learning theory, the experiential learning cycle is actually a learning spiral. When a concrete experience is enriched by reflection, given meaning by thinking, and transformed by action the new experience created becomes richer, broader, and deeper. Further iterations of the cycle continue the exploration and transfer to experiences in other contexts. John Dewey was the first to describe how the learning spiral leads to development; transforming the impulses, feelings, and desires of concrete experience into higher-order purposeful action (Kolb 1984). 
The transformative power of the experiential learning spiral represents the highest culmination of a learning process that can be traced to the organization of life itself. In his classic compilation of the ubiquitous presence of the spiral form in plants and animals of all kinds, Sir Theodore Cook (1914) argues that the spiral is a key to understanding the process of life and the living creations of the human mind. In his concluding chapter he writes, "Throughout our investigations this idea of energy and growth under resistance seems consistently to be connected with the spiral, and we have found that idea recognized in the use of the spiral as a conventional decoration not only by the philosophers of ancient China but even by peoples as old as the Aurignacian civilization of 20,000 years ago. (p. 408)... One of the chief beauties of the spiral as an imaginative conception is that it is always growing, yet never covering the same ground, so that it is not merely an explanation of the past, but is also a prophesy of the future; and while it defines and illuminates what has already happened, it is also leading constantly to new discoveries" (p. 423).

Humberto Maturana discovered the learning spiral in his search for the answer to his question "What is the organization of the living?" What is the pattern of organization that characterizes all living systems and distinguishes them from nonliving physical systems? His answer was that the organization of the nervous system of all living things was basically circular, that living systems are "organized in a closed circular process that allows for evolutionary change in a way that circularity is maintained but not for the loss of the circularity itself." i.e., a spiral. He called this process autopoiesis which means "self-making"; emphasizing the self-referential and self-organizing nature of the network of production processes that produce and transform one another in a continual process of self-making.

With his colleague Francisco Varela, Maturana proceeded to develop the systems theory of cognition arguing that cognition, the process of knowing, was identical with autopoiesis, the process of life (Maturana and Varela 1980). Their definition of cognition, however, was more akin to the holistic concept of experiential learning than the popular definition of cognition as thinking, involving perception, emotion, and action - the entire process of life. In fact, Maturana and Varela argue that cognition is present in all living organisms, even those without brains or nervous systems. The way in which the autopoietic cognition is

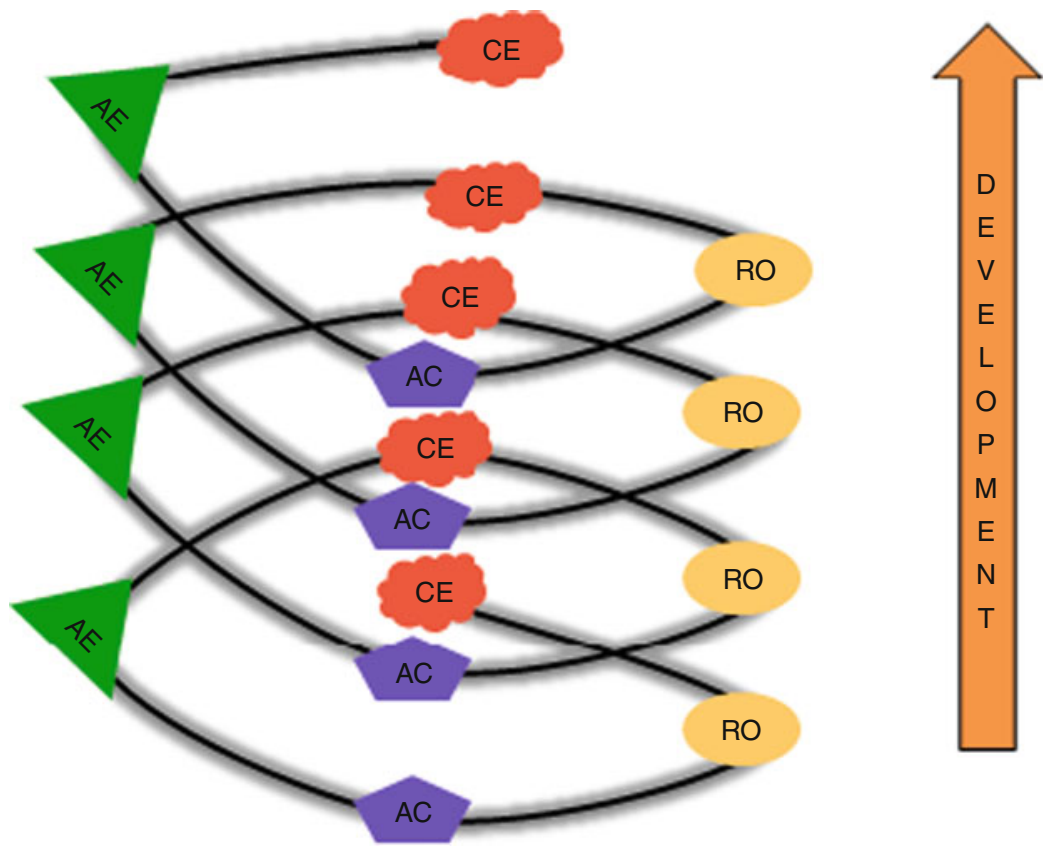

Experiential Learning Spiral. Fig. 1 The spiral of learning from experience 
a learning process is further elaborated in the concept of structural coupling. Structural coupling defines the way a system interacts with its environment, recurrently renewing and recreating itself. The environment does not specify or direct structural changes in the organism because the system is self-referential and selfmaintaining but it triggers them. These structural changes produce changes in the future behavior of the system and its environment. Structural coupling describes the continuing path of the organism's structural changes over time and thus describes the course of the organism's learning and development.

The organization of the mind can be viewed as networks of these autopoietic learning spirals which are embodied in the neuronal networks that cover the surface layer of the neocortex. These neuronal networks are strengthened and enlarged by spirals of learning connecting the major regions of the neocortex. Zull (2002) describes the link between the learning cycle and neuroscience research, suggesting that the spiraling process of experiential learning is related to the way the brain functions: “. . .concrete experiences

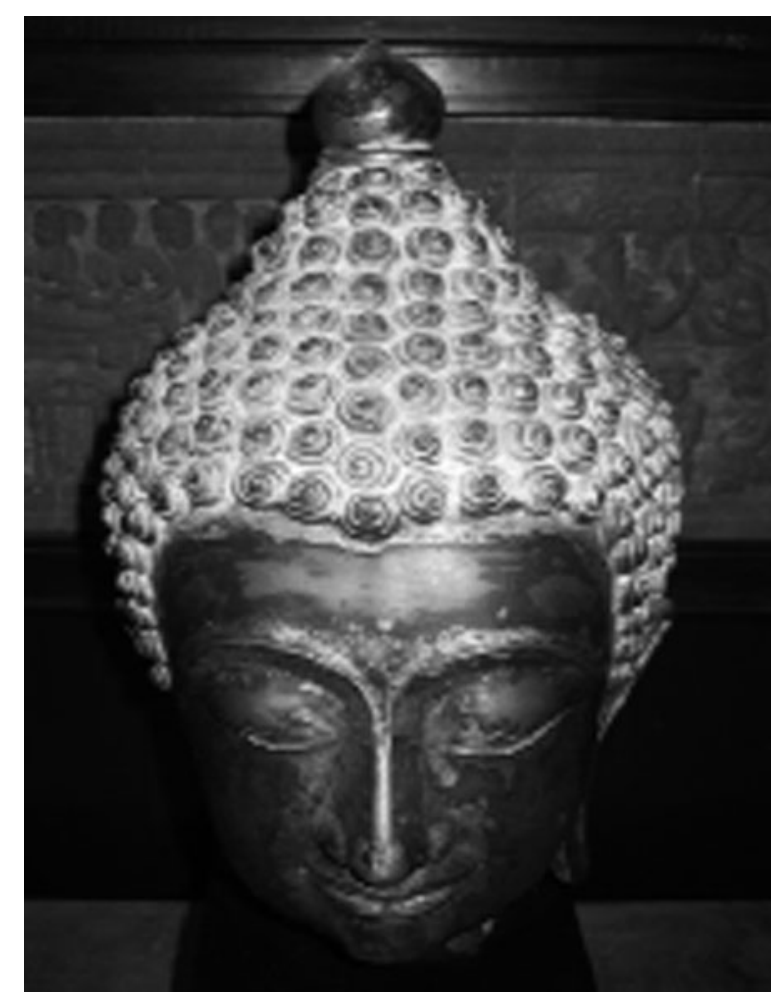

Experiential Learning Spiral. Fig. 2 Buddha with spirals of insight come through the sensory cortex, reflective observation involves the integrative cortex at the back, creating new abstract concepts occurs in the frontal integrative cortex, and active testing involves the motor brain. In other words, the learning cycle arises from the structure of the brain." (Zull 2002, p. 18). A more aesthetic representation of this idea can be found in representations of the Buddha that show his hair coiled in tiny spirals of insight that culminate in enlightenment (see Figs. 1 and 2).

\section{Important Scientific Research and Open Questions}

The learning spiral concept has been widely used in course and curriculum design. Teachers in higher education in many fields have organized their courses as a continuing series of activities that spiral around the learning cycle (Kolb and Kolb 2006). The New Zealand Ministry of Education (2004) has used this spiraling learning process as the framework for the design of middle school curricula, using the learning spiral to promote higher-level learning and transfer to other contexts.

\section{Cross-References}

- Experiential Learning Cycle

- Experiential Learning Spaces

- Experiential Learning Theory

- Kolb's Learning Styles

Learning Identity

- Metacognitive Experiential Learning

\section{References}

Cook, T. A. (1914). The curves of life. London: Constable and Company.

Kolb, D. A. (1984). Experiential learning: Experience as a source of learning and development. Englewood Cliffs: Prentice-Hall.

Kolb, A. Y., \& Kolb, D. A. (2006). Learning styles and learning spaces: A review of the multidisciplinary application of experiential learning in higher education. Chapter 3. In R. Sims \& S. Sims (Eds.), Learning styles and learning: A key to meeting the accountability demands in education (pp. 45-91). Hauppauge: Nova.

Maturana, H., \& Varela, F. (1980). Autopoeisis and cognition. Dordrecht: Reidel.

New Zealand Ministry of Education. (2004). Making meaning: Making a difference. Wellington: Learning Media. www.tki.org. $\mathrm{nz} / \mathrm{r} / \mathrm{health} / \mathrm{cia} / \mathrm{make}$ _meaning/index_e.php.

Zull, J. E. (2002). The art of changing the brain: Enriching teaching by exploring the biology of learning. Sterling: Stylus. 


\section{Experiential Learning Theory}

\author{
Alice Y. KolB ${ }^{1}$, David A. KolB ${ }^{2}$ \\ ${ }^{1}$ Organization Behavior Department, Weatherhead \\ School of Management, Case Western Reserve \\ University, Cleveland Heights, $\mathrm{OH}$, USA \\ ${ }^{2}$ Organization Behavior Department, Case Western \\ Reserve University, Cleveland Heights, OH, USA
}

\section{Synonyms}

Experiential learning; Experience based learning; Learning from experience

\section{Definition}

Experiential Learning Theory (ELT) seeks to pass on the legacy of those twentieth century scholars - notably William James, John Dewey, Kurt Lewin, Jean Piaget, Lev Vygotsky, Carl Jung, Paulo Freire, Carl Rogers, and others - who placed experience at the center of the learning process, envisioning an educational system that was learner centered. ELT is a dynamic view of learning based on a learning cycle driven by the resolution of the dual dialectics of action/reflection and experience/abstraction. It is a holistic theory that defines learning as the major process of human adaptation involving the whole person.

\section{Theoretical Background}

Experiential learning theory draws on the work of prominent twentieth century scholars who gave experience a central role in their theories of human learning and development - notably William James, John Dewey, Kurt Lewin, Jean Piaget, Lev Vygotsky, Carl Jung, Paulo Freire, Carl Rogers, and others (Fig. 1) to develop a dynamic, holistic model of the process of learning from experience and a multi-dimensional model of adult development. From their varied professions and cultural perspectives, these men, while standing at the boundaries of their fields and the traditional educational establishment, have challenged and inspired us to a better way of learning. While their theories have resulted in many profound changes in how we think about learning and education, the full implications of their insights are yet to be realized.

David Kolb (1984) created Experiential Learning Theory to unify the contributions and insights of these scholars into an explicit and coherent framework based both on the common perspectives they share and the unique contributions they have made to our understanding of experiential learning. ELT integrates the

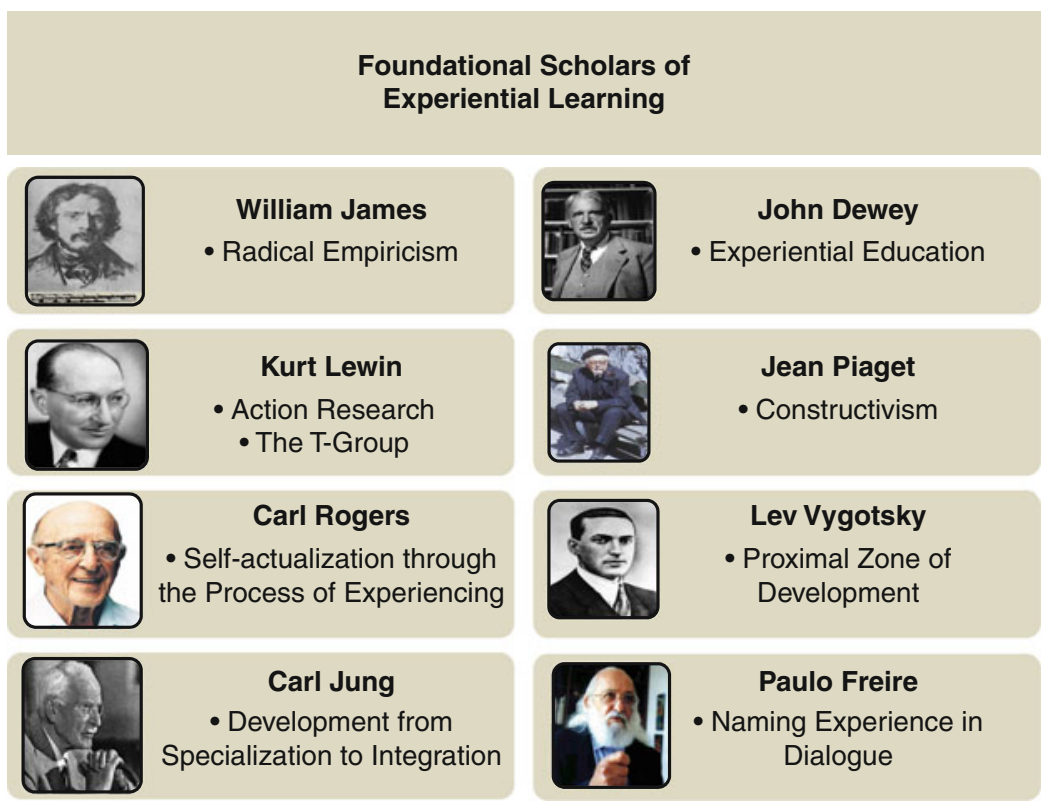

Experiential Learning Theory. Fig. 1 Foundational scholars of experiential learning 
works of the foundational experiential learning scholars around six propositions that they all share:

Learning is best conceived as a process, not in terms of outcomes. Although punctuated by knowledge milestones, learning does not end at an outcome, nor is it always evidenced in performance. Rather, learning occurs through the course of connected experiences. As Dewey suggests, “... education must be conceived as a continuing reconstruction of experience: ... the process and goal of education are one and the same thing" (1897, p. 79).

All learning is re-learning. Learning is best facilitated by a process that draws out the learners' beliefs and ideas about a topic so that they can be examined, tested, and integrated with new, more refined ideas. Piaget called this proposition constructivism - individuals construct their knowledge of the world based on their experience.

Learning requires the resolution of conflicts between dialectically opposed modes of adaptation to the world. Conflict, differences, and disagreement are what drive the learning process. In the process of learning one is called upon to move back and forth between opposing modes of reflection and action and feeling and thinking.

Learning is a holistic process of adaptation. Learning is not just the result of cognition but involves the integrated functioning of the total person - thinking, feeling, perceiving, and behaving. It encompasses other specialized models of adaptation from the scientific method to problem solving, decision making, and creativity. As such, ELT is applicable not only in the formal education classroom but in all arenas of life. The process of learning from experience is ubiquitous, present in human activity everywhere all the time. The holistic nature of the learning process means that it operates at all levels of human society from the individual, to the group, to organizations, and to society as a whole.

Learning results from synergetic transactions between the person and the environment. In Piaget's terms, learning occurs through equilibration of the dialectic processes of assimilating new experiences into existing concepts and accommodating existing concepts to new experience. Following Lewin's famous formula that behavior is a function of the person and the environment, ELT holds that learning is influenced by characteristics of the person and the learning environment.
Learning is the process of creating knowledge. ELT proposes a constructivist theory of learning whereby social knowledge is created and recreated in the personal knowledge of the learner. This stands in contrast to the "transmission" model on which much current educational practice is based where pre-existing fixed ideas are transmitted to the learner.

ELT defines learning as "the process whereby knowledge is created through the transformation of experience. Knowledge results from the combination of grasping and transforming experience" (Kolb 1984, p. 41). The ELT model portrays two dialectically related modes of grasping experience - Concrete Experience (CE) and Abstract Conceptualization (AC) - and two dialectically related modes of transforming experience - Reflective Observation (RO) and Active Experimentation (AE). Experiential learning is a process of constructing knowledge that involves a creative tension among the four learning modes that is responsive to contextual demands. This process is portrayed as an idealized learning cycle or spiral where the learner "touches all the bases" - experiencing, reflecting, thinking, and acting - in a recursive process that is sensitive to the learning situation and what is being learned. Immediate or concrete experiences are the basis for observations and reflections. These reflections are assimilated and distilled into abstract concepts from which new implications for action can be drawn. These implications can be actively tested and serve as guides in creating new experiences (Fig. 2).

In their theories of experiential learning, William James and Paulo Freire describe their views about the integration of these of the concrete/abstract and action/ reflection dialectics. William James proposed radical empiricism as a new philosophy, reality, and mind which resolved the conflicts between nineteenth century rationalism and empiricism, the philosophies of idealism and materialism. For James, everything begins and ends in the continuous flux and flow of experience. His philosophy of radical empiricism was based on two co-equal and dialectically related ways of knowing the world: "knowledge of acquaintance" based on direct perception and "knowledge about" based on mediating conception. In radical empiricism, direct perception has primacy since all concepts derive their validity from connection to sense experience. Concepts, however, have priority in controlling human action because they often enable us to predict the future and achieve 


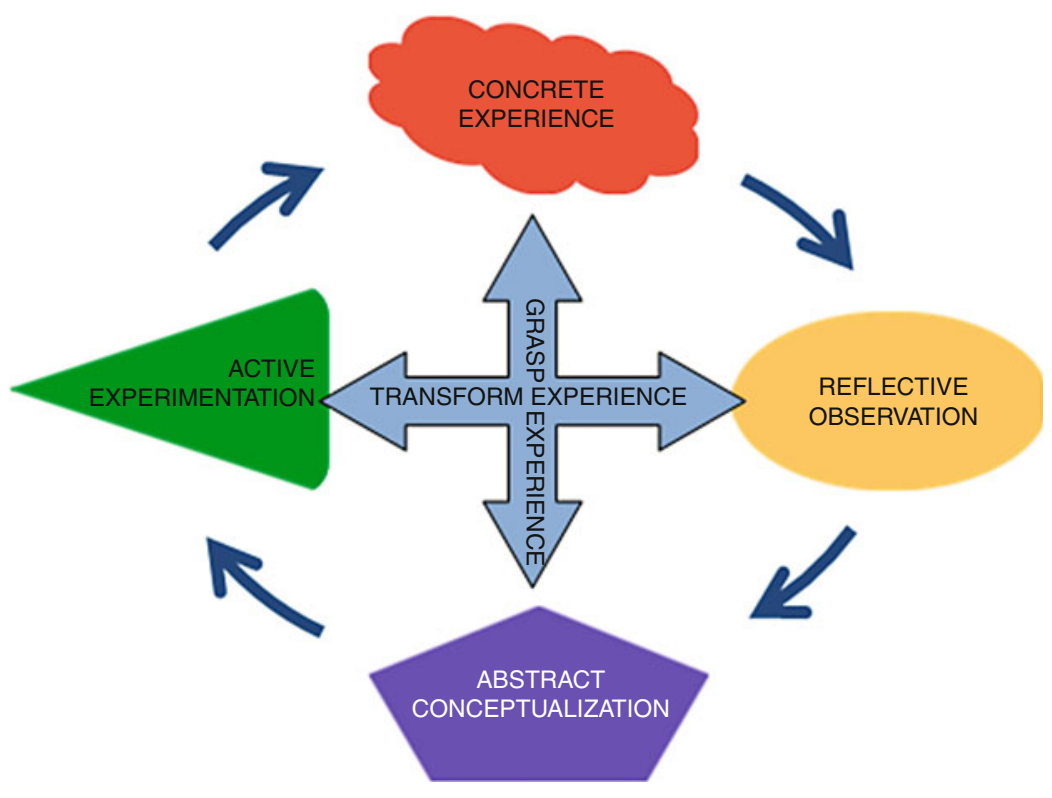

Experiential Learning Theory. Fig. 2 The cycle of experiential learning

our desires. James (1977) draws attention to the importance of this co-equal relationship when he says:

- We thus see clearly what is gained and what is lost when percepts are translated into concepts. Perception is solely of the here and now; conception is of the like and unlike, of the future, and of the past, and of the far away. But this map of what surrounds the present, like all maps, is only a surface; its features are but abstract signs and symbols of things that in themselves are concrete bits of sensible experience. We have but to weigh extent against content, thickness against spread, and we see that for some purposes the one, for other purposes the other, has the higher value. Who can decide off-hand which is absolutely better to live and to understand life? We must do both alternately, and a man can no more limit himself to either than a pair of scissors can cut with a single one of its blades (p. 243).

While the conceptualizing/experiencing dialectic described by James is recognized by the Brazilian educator Paulo Freire, by stressing the importance of naming one's own experience in dialogue with others, he and other critical theorists give primary emphasis to praxis, the transformative dialectic between reflection and action - reflection informed by action and action informed by reflection. He writes powerfully about the dynamics of this dialectic:
- As we attempt to analyze dialogue as a human phenomenon... Within the word we find two dimensions, reflection and action, in such radical interaction that if one is sacrificed - even in part - the other immediately suffers.... When a word is deprived of its dimension of action, reflection automatically suffers as well; and the word is changed into idle chatter, into verbalism, into an alienated and alienating 'blah'.... On the other hand, if action is emphasized exclusively, to the detriment of reflection, the word is converted into activism. The latter action for action's sake negates the true praxis and makes dialogue impossible (1993, pp. 75-78).

\section{Important Scientific Research and Open Questions}

ELT was developed following Lewin's plan for the creation of scientific knowledge by conceptualizing phenomena through formal, explicit, testable theory. In his approach, before a system can be fully useful the concepts in it have to be defined in a way that (1) permits the treatment of both the qualitative and quantitative aspects of phenomena in a single system, (2) adequately represents the conditional-genetic (or causal) attributes of phenomena, (3) facilitates the measurement (or operational definition) of these attributes, and (4) allows both generalization to universal 
laws and concrete treatment of the individual case. A theory developed by this process can be a powerful instrument for stimulating and focusing scholarly research conversation.

Since its first statement in 1971 there have been many studies using ELT to advance the theory and practice of experiential learning. Since ELT is a holistic theory of learning that identifies learning style differences among different academic specialties, it is not surprising to see that ELT research is highly interdisciplinary, addressing learning and educational issues in many fields. An analysis of the 1,004 entries in the 1999 bibliography shows 207 studies in management, 430 in education, 104 in information science, 101 in psychology, 72 in medicine, 63 in nursing, 22 in accounting, and 5 in law. About 55\% of this research has appeared in refereed journal articles, $20 \%$ in doctoral dissertations, $10 \%$ in books and book chapters, and $15 \%$ in conference proceedings, research reports, and others. Since 2000, ELT research in these fields around the world has more than doubled. The current experiential learning theory bibliographies (Kolb and Kolb 2010a, b) include over 2,500 entries.

Included are research studies from every region of the world with many contributions coming from the USA, Canada, Brazil, the UK, China, India, Australia, Japan, Norway, Finland, Sweden, the Netherlands, and Thailand. These studies support the cross-cultural validity of ELT and the KLSI and also support practical applicability across cultures. The KLSI has been translated into many languages including, English, Spanish, French, Portuguese, Arabic, Russian, Dutch, German, Swedish, Chinese, Romanian, Persian, Thai, and Japanese. The pragmatic value of the holistic ELT framework for understanding cultural differences has been shown in a number of studies on cross-cultural management.

There have been two comprehensive reviews of the ELT literature, one qualitative and one quantitative. In 1991, Hickox extensively reviewed the theoretical origins of ELT and qualitatively analyzed 81 studies that focused on the application of the ELT model as well as on the application of the concept of learning style in accounting and business education, helping professions, medical professions, post-secondary education, and teacher education. She concluded that overall $61.7 \%$ of the studies supported ELT, $16.1 \%$ showed mixed support, and $22.2 \%$ did not support ELT. In 1994, Iliff conducted a meta-analysis of 101 quantitative LSI studies culled from 275 dissertations and 624 articles that were qualitative, theoretical, and quantitative studies of ELT and the Kolb Learning Style Inventory (KLSI, Kolb 2005). Using Hickox's evaluation format he found that 49 studies showed strong support for the LSI, 40 showed mixed support, and 12 studies showed no support. About half of the 101 studies reported sufficient data on the LSI scales to compute effect sizes via meta-analysis. Most studies reported correlations that fell in the $0.2-0.5$ range for the LSI scales. In conclusion, Iliff suggested that the magnitude of these statistics is not sufficient to meet standards of predictive validity, while noting that the LSI was not intended to be a predictive psychological test like IQ, GRE, or GMAT. The LSI was originally developed as a self-assessment exercise and a means for construct validation of ELT. Judged by the standards of construct validity ELT has been widely accepted as a useful framework for learning centered educational innovation, including instructional design, curriculum development, and life-long learning. Academic field and job classification studies viewed as a whole also show a pattern of results consistent with the ELT structure of knowledge theory. Most of the debate and critique in the ELT/LSI literature has centered on the psychometric properties of the LSI. Results from this research have been of great value in revising the LSI in 1985 , in 1999, and again in 2005 . Recent critique has been more focused on the theory than the instrument examining the intellectual origins and underlying assumptions of ELT from what might be called a critical theory perspective where the theory is seen as individualistic, cognitivist, and technological (e.g., Vince 1998; Holman 1997; Hopkins 1993). Kayes (2002) has reviewed these and other critics of ELT and offered his own critique of the critics. He suggests that critics have overlooked the role of Vygotsky's social constructivist learning theory in the ELT theory of development and the role of personal knowledge and social knowledge in experiential learning. He proposes an extension of ELT based on Lacan's poststructuralist analysis that elaborates the fracture between personal and social knowledge and the role that language plays in shaping experience. 


\section{Cross-References}

- Dewey, John

- Experiential Learning Spaces

- Experiential Learning Spiral

- James, William (1842-1910)

- Kolb's Learning Styles

- Kurt Lewin

Learning Identity

- Learning Style

- Meta-cognitive Experiential Learning

- Piaget, Jean

\section{References}

Freire, P. (1992). Pedagogy of the oppressed. New York: Continuum. Freire, P. (1993). The pedagogy of the oppressed. New York: Continuum. Holman, D., Pavlica, K., \& Thorpe, R. (1997). Rethinking Kolb's theory of experiential learning in management education: The contribution of social constructionism and activity theory. Management Learning, 28(2), 135-148.

Hopkins, R. (1993). David Kolb's experiential learning-machine. Journal of Phenomenological Psychology, 24(1), 46-62.

James, W. (1977). Percept and concept: The import of concepts. In J. McDermott (Ed.), The writings of William James. Chicago: University of Chicago Press.

Kayes, D. C. (2002). Experiential learning and its critics: Preserving the role of experience in management education. Academy of Management Learning and Education, 1(2), 137-149.

Kolb, A. Y., \& Kolb, D. A. (2005). The Kolb learning style inventoryversion 3.1: 2005 Technical specifications. Boston: Hay Resources Direct.

Kolb, A. Y., \& Kolb, D. A. (2010a). Experiential learning theory bibliography: 1971-2005. Cleveland: Experience Basd Learning Systems. www.learningfromexperience.com.

Kolb, A. Y., \& Kolb, D. A. (2010b). Experiential learning theory bibliography: Recent research 2006-2010. Cleveland: Experience Basd Learning Systems. www.learningfromexperience.com.

Kolb, D. A. (1984). Experiential learning: Experience as the source of learning and development. Englewood Cliffs: Prentice-Hall.

Kolb, D. A. (2005). The Kolb learning style inventory - version 3.1. Boston: Hay Resources Direct.

Vince, R. (1998). Behind and beyond Kolb's learning cycle. Journal of Management Education, 22(3), 304-319.

\section{Experiential Methodology}

The teacher links the linguistic materials to the everyday use of learners' language.

\section{Experiential/Significant Learning}

\author{
Renate Motschnig ${ }^{1}$, Jeffrey H. D. Cornelius-White ${ }^{2}$ \\ ${ }^{1}$ Computer Science Didactics and Learning Research \\ Center, University of Vienna, Vienna, Austria \\ ${ }^{2}$ Department of Counseling, Leadership, and Special \\ Education, Missouri State University, Springfield, \\ MO, USA
}

\section{Synonyms}

Meaningful learning; Person-centered learning; Whole-person learning

\section{Definition}

Carl R. Rogers contrasts learning that involves the mind only, such as rote learning of facts, with "significant, meaningful, experiential learning." As an example of the latter, he describes the experience when "the toddler touches the warm radiator and learns for herself the meaning of the word hot; she has learned a future caution in regard to all similar radiators [...] in a significant, involved way that will not soon be forgotten." By significant learning, Rogers means "learning that is more than an accumulation of facts. It is learning that makes a difference - in the individual's behavior, in the course of action he chooses in the future, in his attitudes, and in his personality. It is a pervasive learning, which is not just an accretion of knowledge, but which interpenetrates with every portion of his experience." (Rogers 1961, p. 280)

According to Rogers (1983, p. 20), the elements that are involved in such significant or experiential learning are as follows:

- There is a quality of personal involvement - the whole person in both $>$ feeling and cognitive aspects being in the learning event.

- Learning is self-initiated. Even when the impetus or stimulus comes from the outside, the sense of discovery, the reaching out, of grasping and comprehending, comes from within.

- Learning is pervasive. It makes a difference in the behavior, the attitudes, perhaps even the personality of the learner. 
- Progress is evaluated by the learner. She knows whether it is meeting her need, whether it leads toward what she wants to know, whether it illuminates the dark area of ignorance she is experiencing. The locus of evaluation resides definitely within the learner.

- Its essence is meaning. When such learning takes place, the element of meaning to the learner is built into the whole experience. (Rogers 1983, p. 20)

In a nutshell, "significant learning combines the logical and the intuitive, the intellect and the feelings, the concept and the experience, the idea and the meaning. When we learn in that way, we are whole." (Rogers 1983, p. 20).

\section{Theoretical Background}

Rogers derived his educational direction from his experiences as a student and subsequently his practice, facilitation, and research in psychotherapy. Rogers made it clear that his findings in the area of education would be valid only if the goal of education was not as much to increase the learning of established, factual information but rather to facilitate something more holistic. He believed the goal of education should be to promote creativity, to free the mind, and welcome self-direction in the development of democratic citizens who could be trusted to think, feel, and act for themselves.

As a student at Teachers College of Columbia University, Rogers took "a course in clinical psychology under the guidance of Leta Hollingworth, of whom he significantly remarked that she combined the qualities of a warm human being with those of a competent research worker." (Thorne 2002, p. 7) Equally important was Rogers' contact with William Heard Kilpatrick who was a former student of John Dewey and who persuasively and powerfully disseminated Dewey's views on progressive education (Thorne 2002).

As a professor, Rogers gradually granted students more and more freedom. This included letting students choose their own goals and participate in all aspects of learning, including their assessment such that they could suggest grades and subsequently discuss them with the "teacher." Since this style of education focused on students' learning much more than on teaching in any traditional sense, the "teacher" more appropriately came to be called a facilitator of learning.
His or her job was, in the first place, to foster a constructive atmosphere in class and, in addition, to be a resourceful person, making various kinds of resources such as books, videos, communities, etc., as well as himself/herself available to students to facilitate their learning. If there was teaching in the classroom, it was requested by students and was never imposed on them because Rogers never tried to have people learn things they didn't want to learn.

In order to understand the meaning of Rogers's notion of experiential learning, it is helpful to refer to the process that led him to distinguish significant, experiential learning. Rogers's lasting and ever repeated experience of endeavoring to be helpful to individuals in distress convinced him that persons have a basically positive direction. When he could sensitively understand the feelings they expressed and was able to accept them as persons in their own right, he found that they tended to move in directions that could be described as constructive, moving toward actualization, growing toward maturity and socialization. While Rogers was well aware that out of defensiveness and fear individuals behave in ways that are incredibly cruel, destructive, and antisocial, he experienced that when he created an honest, empathic, and accepting climate, strongly positive directional tendencies existed in individuals at the deepest levels (Rogers 1961, p. $26-27$ ).

To Rogers, his own experience meant the highest authority and it was to experience that he had to return again and again, to discover a closer approximation to truth. Rogers (1961, p. 25) wrote: "My experience is not authoritative because it is infallible. It is the basis of authority because it can always be checked in new, primary ways. In this way, its frequent error or fallibility is always open to correction." From this follows Rogers' conviction and corresponding attitude as a facilitator, namely, that there is no philosophy or set of principles, which he would encourage or persuade others to accept. Rogers stated (Rogers 1961, p. 27): "I can only try to live by $m y$ interpretation of the current meaning of my experience, and try to give others the permission and freedom to develop their own inward freedom and thus their own meaningful interpretation of their own experience."

The basic motive upon which Rogers relied for significant learning to happen was that in each person there is a directional, forward-moving tendency, referred to as an actualizing tendency. Students 
"who are in real contact with life problems wish to learn, want to grow, seek to find out, hope to master, desire to create" (Rogers 1961, p. 289). Based on Rogers' findings in psychotherapy where he and his coworkers lived and researched the necessary and sufficient conditions for personal growth to take place, the task of the teacher or better facilitator of learning was to provide these attitudinal conditions and thereby to create a facilitating classroom climate in which significant learning can happen. According to Rogers (1983, p. 121), "the initiation of such learning rests not upon the teaching skills of the leader, not upon scholarly knowledge of the field, not upon curricular planning, not upon use of audiovisual aids, not upon the programmed learning used, not upon lectures and presentations, not upon an abundance of books, though each of these might at one time or another be utilized as an important resource. No, the facilitation of significant learning rests upon certain attitudinal qualities that exist in the personal relationship between the facilitator and the learner." (Rogers 1983, S. 121)

But what are the interpersonal conditions ( $\triangleright$ Person-Centered Learning) or qualities that facilitate significant learning? To Rogers, the most basic attitude is realness or genuineness or congruence. This means that the facilitator is a real person with the feelings he or she is experiencing available to his or her awareness and entering into a direct personal encounter with the learner, not one in which the facilitator simply plays a professional role or puts on a front or a façade. Another essential attitude is one of accepting or prizing the learner as a person of unconditional worth and caring for him or her in a non-possessive, nonjudgmental way. Acceptance is a basic trust in the actualizing tendency - a belief that this other person is fundamentally trustworthy no matter what his or her feelings, opinions, ideas, problems are at this moment. The third quality that promotes experiential learning is $\mathbf{D}$ empathic understanding the facilitator's sensitivity to understand the learner's reactions from their point of view, to understand how some situation feels and seems to the learners without wanting to analyze or to judge them. It is essential that the facilitator communicate all three qualities to learners in such a way that the learners perceive them at least to some degree.

When it is being facilitated, experiential learning is likely to involve some of the following elements:
- Building upon problems perceived as having meaning and relevance for students

- Provision and accessibility of material resources, such as tutorials, books, or training information

- Providing personal resources, using the community, and making oneself available as a resource

- Peer teaching and peer evaluation

- Use of learning contracts

- Cooperative learning in small groups

- Conducting inquiry: Helping students to become inquirers working toward scientific discovery

- Self-evaluation, reflection, and taking account of the evaluation of one's own learning

- Encounter groups (see below).

Encounter groups are particularly interesting not only for students but also for teachers and administrators who aim to increase their self-understanding and facilitative capacities by directly experiencing significant, experiential learning in a group setting (Rogers 1970). Very briefly, an encounter group facilitator's function is to facilitate the group members' expression in a climate based on realness, acceptance, and empathic understanding. After an initial period of "milling around," an increasingly free, unmasked, direct, spontaneous communication tends to occur between members of the group. They express hitherto hidden feelings and aspects of themselves and receive feedback - both positive and negative. As a result, many persons become much more facilitative in relationships with others. When the process is a fruitful one, it is a deeply personal experience resulting in more direct person-to-person communication, increased selfunderstanding, more realness and independence in the individual, and an increased understanding and acceptance of others (Rogers 1978).These are the same attitudes as those that are highly conducive to experiential learning.

\section{Important Scientific Research and Open Questions}

In his book "Freedom to learn for the 1980s," Rogers (1983) devoted a whole chapter to "Researching Person-centered Issues in Education." He provided a rich reference list and included findings by David Aspy \& Flora Roebuck in the United States and by Reinhard and Annemarie Tausch in Germany. David Aspy and Flora Roebuck conducted research with the 
National Consortium for Humanizing Education (NCHE). NCHE activities focused on interpersonal relationships in classrooms in all levels of schools from 42 states within the United States and seven foreign countries over a period of 17 years. Activities employed subjective (phenomenological) and scientific procedures to examine the relationship between Rogers' facilitative conditions (realness/genuineness, acceptance, empathic understanding) and factors such as attitudes (toward self, school, or others) discipline problems, physical health, attendance, I.Q. changes, and cognitive growth. The findings can be summarized as follows: "Students learn more and behave better when they receive high levels of understanding, caring, and genuineness, than when they are given low levels of them." (Aspy and Roebuck in Rogers 1983, p. 199). These findings have been confirmed and further refined in a recent extensive meta-analysis of learner-centered education conducted by Jeffrey Cornelius-White (Cornelius-White and Harbaugh 2010). In Germany, Tausch \& Tausch achieved corresponding results. All their studies indicated that low ratings on Rogers' facilitative conditions and on non-directive facilitation along with high ratings on directive leading were accompanied by lower levels of pupil intellectual performance and significantly negative emotional experiences. In a study on the effects of encounter groups, the Tauschs found $73 \%$ of the teachers who had participated in a two and a half day encounter group had long-lasting changes in their personalities, leading to improved self-concepts and changes in their teaching behavior. Their pupils became more trusting of the teacher, enjoyed more self-determination, and had fewer discipline problems. Ninety-nine percent of the teachers who had participated at the encounter groups said that such groups were helpful for their work at school (Tausch in Rogers 1983, p. 218).

Throughout the last decade (starting in 2000), Renate Motschnig and her colleagues at the Computer Science and Learning Research Center at the University of Vienna, Austria, thoughtfully integrated Rogers' person-centered, experiential learning with web-based technology, resulting in Person-Centered, technology enhanced Learning (PCeL). Furthermore, they have conducted and studied Significant Learning Communities (Motschnig-Pitrik 2008), which are encounter groups with online contact of participants being provided between encounter sessions. More than 70 studies using qualitative and quantitative methods as well as action research in an academic, blendedlearning context showed that, given a facilitative climate is perceived by students, web-technology can be used as an ally in enhancing learning. In comparison with traditional classes, learning in such environments shows improvements in the dimensions of active participation, increased motivation (due to the constructive atmosphere provided), self-initiated contributions, team skills, and communicative competence, particularly in active listening and giving and receiving feedback. Although further research on experiential learning in groups is necessary, students' feelings of community - as measured by an instrument developed by Godfrey Barrett-Lennard (2005) - rose significantly as a result of attending classes that were facilitated in a person-centered way.

Major open questions include:

- How can effective programs that continuously support educators in developing, strengthening, and passing on facilitative attitudes be put in place?

- How can experiential and cognitive learning best be integrated?

- How much closure should be achieved from experiential learning and to what degree should open processes be allowed to continue unfolding gradually?

- How can innovative technological developments be integrated thoughtfully and sensitively into classes emphasizing experiential learning?

- Can the constructive, communicative basis (Motschnig and Nykl 2009) underlying significant learning be shown to be cross-culturally helpful and effective, in particular, in dealing with diversity?

\section{Cross-References}

- Action Research on Learning

- Computer-Supported Collaborative Learning

- Constructivist Learning

- Experiential Learning Theory

Interactive Learning Environments

- Rogers, Carl R. (1902-1987)

- Student-Centered Learning

\section{References}

Barrett-Lennard, G. T. (2005). Relationship at the centre, healing in a troubled world. Philadelphia: Whurr. 
Cornelius-White, J. H. D., \& Harbaugh, A. P. (2010). Learner-centered instruction: Building relationships for student success. Thousand Oaks: Sage.

Motschnig-Pitrik, R. (2008). Significant learning communities as environments for actualising human potentials. International Journal of Knowledge and Learning (IJKL), 4(4), 383-397.

Motschnig, R., \& Nykl, L. (2009). Konstruktive Kommunikation Sich und andere verstehen durch personenzentrierte Interaktion. Stuttgart: Klett-Cotta.

Rogers, C. R. (1961). On becoming a person - a psychotherapists view of psychotherapy. London: Constable.

Rogers, C. R. (1970). Carl Rogers on encounter groups. New York: Harper \& Row.

Rogers, C. R. (1978). Carl Rogers on Personal Power: Inner Strength and its Revolutionary Impact. London: Constable.

Rogers, C. R. (1983). Freedom to learn for the 80's. Columbus: Charles E. Merrill Publishing Company/A Bell\&Howell Company.

Thorne, B. (2002). Bibliographical introduction. In C. R. Rogers \& D. E. Russel (Eds.), Carl Rogers the quiet revolutionary, an oral history. Roseville: Penmarin Books.

\section{Experiment}

Laboratory Learning

\section{Experimental}

- Field Experiments in Learning Research

\section{Experimental and Quasi- Experimental Designs for Research on Learning}

\author{
Norbert M. SeEL
}

Department of Education, University of Freiburg, Freiburg, Germany

\section{Synonyms}

Research designs

\section{Definition}

Research on learning applies various designs which refer to plans that outline how information is to be gathered for testing a hypothesis or theoretical assumption. Research designs are the heart of quantitative research. They include systematic observations, measures, treatments, their random assignment to groups, and time. Accordingly, research designs include identifying the data gathering method(s), the instruments to be used or created for assessment, how the instruments will be administered, and how the information will be organized and analyzed in accordance with the subject to be investigated. Among the various designs to consider in the area of research on learning are

- Experimental designs

- Quasi-experimental designs

- Nonexperimental designs

Each design offers its particular advantages and disadvantages concerning validity, reliability, and feasibility. Although all experiments share common features, their applications vary in accordance with the type of design used.

\section{Theoretical Background}

Experiments are a widely used methodology for advancing causal knowledge in the physical and life sciences. Since the end of the nineteenth century, experimental research emerged in psychology and, in consequence, most studies on human and animal learning were experimental by nature. Nonetheless, until today we easily can find considerable resistance against experiments in research on learning. Resistance concerning experimental evidence often centers on an appeal to "realism" and generalizability. This skepticism often results in preferring nonexperimental research designs combined with gathering qualitative data.

However, controlled variation is the essence and major characteristic of empirical research and the foundation of empirically assured scientific knowledge. Only true experiments allow tight control of causal relationships between variables. Experimental designs aim at the investigation of something that naturally occurs in nature through the deliberate manipulation of specific variables (Kirk 2003). This manipulation is usually referred to as the treatment in an experiment. Scientists conduct experiments in order to determine how a treatment affects necessarily a change in natural orders. Actually, discovering causal relationships is the key to experimental research in general. This means, in other words, the strong relationship between 
a certain treatment $\mathrm{X}$ which alone produces the effect $\mathrm{Y}$, so that $\mathrm{Y}$ if and only if $\mathrm{X}$.

Not only in research on learning but rather in general, the term experiment refers to a variety of research designs that focus on relatively permanent changes in behavior or in knowledge and skills that occur as a direct result of specific experiences. Accordingly, pretest-posttest (before-after) as well as group comparisons is used to measure the effects of a treatment on learning.

Basically, a distinction can be made between controlled experiments and field experiments. The controlled experiment is a type of experiment in which sampling of the subjects occurs randomly and subjects are randomly assigned to either an experimental group (i.e., the group that experiences the treatment) or a control group (i.e., the group that does not experience the treatment).

Group 1: Pretest -..--->> Treatment -...-- -> Posttest Group 2: Pretest -......-.

In addition, influencing extraneous (for example, environmental) variables are also controlled in order to isolate the effects of the treatment. In contrast to controlled laboratory experiments, field experiments are conducted in natural settings. Field experiments, like lab experiments, generally randomize the sampling of subjects and their assignment into treatment and control groups and compare outcomes between these groups. In contrast, field studies are a type of research where subjects are assigned to experimental (and control groups) in a nonrandom fashion. In accordance with Creswell (2005), we can distinguish between different types of experimental designs:

True experiments comprise the most rigorous and strong control of treatment effects due to random assignment of subjects to different experimental variations in comparison with a control group. Whereas true experiments are regularly conducted in the laboratory, many experimental situations may occur in which researchers have to work with given groups (e.g., school classes) so that an assignment of participants to randomized groups is not possible. These experimental settings are called quasi-experiments, which include assignment, but not random assignment of the subjects to either experimental or the control condition. A quasi-experiment considers a given situation as an experiment even though it is not a true experiment. The independent variable may not be manipulated by the researcher, treatment and control groups may not be randomized or matched, or there may be no control group. In some experimental situations, it is not sufficient to learn about the effects of a single treatment on outcomes but rather several treatments may provide a better explanation for the observed effects. So-called factorial designs fit with this condition because they allow investigating the simultaneous effects of two or more treatments on outcomes. Up to now, the research designs have included comparisons between the experimental and control group; therefore, they can be named "between-group designs." However, in some situations the number of subjects may be limited so that only one group can be assigned to an experimental treatment. In these cases, only comparisons within a group are possible and we speak, in consequence, about "within-group experimental designs" which can be time series, repeated measures, and singlegroup designs. Time series designs are especially adequate for investigating learning processes and consist of studying one group over time. Creswell (2005) distinguishes between interrupted and equivalent time series designs, which he describes as follows (Table 1).

Another within-group experimental design which fits best with research on cumulative learning is the repeated measures design. All subjects in a single group participate in multiple experimental treatments over time and the group's performance under one treatment is compared with its performance under another subsequent treatment. The steps in this design are shown in Table 2.

Finally, single-subject designs involve the study of single individuals $(\mathrm{N}=1)$ over a baseline period followed by a treatment. The idea of these designs is to determine whether a treatment affects the behavior of a subject by observing this subject over a prolonged period of time and recording the behavior before and after the treatment (see for more details about variations of single-subject design: Creswell 2005).

In sum, the most common designs in psychology and in research in learning are:

- Between-group designs

- Controlled ("true") experiments (pre- and posttest, posttest only) 
Experimental and Quasi-Experimental Designs for Research on Learning. Table 1 Time series experimental designs (Creswell 2005, p. 302)

\begin{tabular}{|c|c|c|c|c|c|c|c|}
\hline \multicolumn{8}{|c|}{ Interrupted time series design } \\
\hline \multicolumn{8}{|c|}{ Time } \\
\hline $\begin{array}{l}\text { Select } \\
\text { Participants } \\
\text { for Group }\end{array}$ & $\begin{array}{l}\text { Observation } \mathrm{O}_{1} \\
\text { (Pretest } \\
\text { Measure) }\end{array}$ & $\begin{array}{l}\text { Observation } \\
\mathrm{O}_{2}\end{array}$ & $\begin{array}{l}\text { Observation } \\
\mathrm{O}_{3}\end{array}$ & $\begin{array}{l}\text { Treatment } \\
X\end{array}$ & $\begin{array}{l}\text { Observation } \mathrm{O}_{4} \\
\text { (Posttest } \\
\text { measure) }\end{array}$ & $\begin{array}{l}\text { Observation } \\
\mathrm{O}_{5}\end{array}$ & $\begin{array}{l}\text { Observation } \\
\mathrm{O}_{6}\end{array}$ \\
\hline \multicolumn{8}{|c|}{ Equivalent time series design } \\
\hline \multicolumn{8}{|c|}{ Time } \\
\hline $\begin{array}{l}\text { Select } \\
\text { Participants } \\
\text { for Group }\end{array}$ & Observation $\mathrm{O}_{1}$ & Treatment & $\begin{array}{l}\text { Observation } \\
\mathrm{O}_{2}\end{array}$ & Treatment & Observation $\mathrm{O}_{3}$ & Treatment & $\begin{array}{l}\text { Observation } \\
\mathrm{O}_{4}\end{array}$ \\
\hline
\end{tabular}

Experimental and Quasi-Experimental Designs for Research on Learning. Table 2 The repeated measures design (Creswell 2005, p. 303)

\begin{tabular}{|l|l|l|l|l|l|l|}
\hline The repeated measures design \\
\hline \multicolumn{7}{|c|}{ Time } \\
\hline Select Participants for Group & Observation $\mathrm{O}_{1}$ & Treatment \#1 & Observation $\mathrm{O}_{2}$ & Treatment \#2 & Observation $\mathrm{O}_{3}$ & $\ldots$. \\
\hline
\end{tabular}

- Quasi-experiments (pre- and posttest, posttest only)

- Factorial designs

- Within-group or individual designs

- Time series (interrupted, equivalent)

- Repeated measures experiments

- Single-subject experiments

In research designs, various types of variables are included: First of all, there is the independent variable with the focus on the treatment or what is manipulated systematically in an experiment. What is observed from the effects of a treatment in an experiment is called the dependent variable. A third group of variables is called controlled variables; these refer to factors that remain constant throughout an experiment and must be controlled in order to find the unique effects of a treatment. Finally, there are extraneous variables which can influence the relationship between the independent and dependent variables of an experiment without any chance to control their effects. Clearly, these effects of extraneous variables are undesired because they add errors to an experiment. Taking relevant extraneous variables into consideration, Campbell and Stanley (1963) identified important threats to the internal and external validity of various research designs. On the one side, there are factors which can affect how valid an investigation is by itself; on the other side, there are factors which can affect how well the results of a study apply to the target population. In their seminal contribution to the field, Campbell and Stanley (1963) have addressed the following threats to internal validity (see also Cook and Campbell 1979).

History: Outside events occurring during the course of the experiment or between repeated measures of the dependent variable may have an influence on the results. 
Maturation: Change due to aging or development, either between or within groups.

Instrumentation: The reliability of the instrument may change in calibration or from change in human ability to measure differences (due to fatigue, experience, etc.).

Testing: Participants may become familiar with the measures and remember responses for later test.

Statistical regression: When researchers select subjects for a group based on extreme scores, a more or less strong tendency to regress toward mean makes scores higher or lower in subsequent measures.

Selection: The participants in groups may be unlike in some way, so they will respond in different ways to the independent variable. This is mostly a risk for quasi-experimental designs, in which nonrandom assignment is used.

Mortality: Participants drop out during the experiments for several reasons so that drawing conclusions from scores may become difficult.

Interaction with selection: Two or more threats mentioned thus far can interact. For example, a selection-maturation interaction may occur when different ages of groups can change at different rates due to maturation.

Experimenter bias: Expectations of an outcome may inadvertently influence participant or cause the experimenter to view data in a different way.

Placebo effect: Improvement due to expectation rather than the treatment itself; can occur when participants receive a treatment that they consider likely to be beneficial.

Hawthorne effect: When members of the treatment group change in terms of the dependent variable because their participation in the study makes them feel special - so they act differently, regardless of the treatment.

Contamination: When the comparison group is in some way affected by, or affects, the treatment group, causing an increase of efforts. Contamination is also known as compensatory rivalry.

Additionally, Campbell and Stanley (1963) also listed some important threats to external validity, that is, factors which can affect whether and how well results of a study can be applied or generalized to the target population. A generalization of empirical data with confidence to the population is dependent on the following.
Selection bias (interaction of selection and treatment): The sample is not representative of the population. Then it is not possible to generalize beyond the groups in the experiment.

Reactive effects of experimental arrangements: Results can be traced back to the experimental setting but not to the particular treatment. Controlling confounding variables is a suitable way to exclude reactive effect but there is always a trade-off between control and external validity. When designing an experiment, a decision must be made what is most important for the particular research.

Multiple treatment interference (or interaction of history and treatment): This threat to external validity may emerge when several treatments are subsequently realized in an experiment or when an experiment is done at a special point of time and may not produce similar results when conducted earlier. This can be accounted for by using a Latin square, where all the groups get each treatment, but in different orders.

A more detailed analysis of threats to external validity has been provided by Bracht and Glass (1968).

Since 1960s it has become a good practice for improving internal and external validity of experiments by checking the various experimental and quasi-experimental designs on the basis of the aforementioned factors which affect internal and external validity. For instance, all designs for true experiments (i.e., the pretest-posttest control group design, the Solomon four group design, and the posttest-only control group design) control all sources of internal invalidity but not (or partially) the sources of external invalidity. Due to the lack of randomization quasiexperimental designs (for example, time series experiments and counterbalanced designs) are even weaker in the possible control of the threats to internal and external validity. However, there are some quasiexperimental designs (e.g., the separate-sample pretest-posttest designs) which control all threats to external validity and therefore permit a valid generalization of experimental results to the target population.

\section{Important Scientific Research and Open Questions}

Since the days of Fisher (1925) the randomized "true experiment" is considered the gold standard for causal inferences from data. Indeed, randomization of participants and their random assignment to treatments has 
long been considered as the most powerful method of control, so much so that it became the distinguishing characteristic between experimental and other types of research (Shadish et al. 2002). However, sometimes particular circumstances do not permit a true experiment. Then, a quasi-experiment is chosen. In general, only experimental and quasi-experimental research designs allow to control and manipulate (at least some of) the factors of interest in order to find evidence for "causal" relations. In contrast, there is little to be gained from a single, nonexperimental study. Many standard experimental designs are used in the study of animal and human learning (see Wilcox 2003). Among the various quasi-experimental designs is one that rivals the true experiment: the interrupted timeseries design. It has become the standard method of causal analysis in applied research on learning.

The control of extraneous variables is a fundamental condition for causal interpretations in research. Many researchers typically use betweengroup and within-group designs where they deal with extraneous variables in different ways. These variables may be held constant, counterbalanced, or randomized. Aiming at internal validity of their studies, researchers attempt to hold constant as many variables as possible, although it is recognized that this may result in a limited generalization of the conclusions from the specific conditions used in the experiment to the target population. Variables that are likely to have an effect on performance will be counterbalanced; all other variables will be randomized. To reduce the effect of intraindividual differences, many researchers typically use repeated measures designs. In a repeated measures design, each of the participants receives each of condition. Because of potential carryover effects, different participants receive the conditions in different orders which may be counterbalanced or randomized. Other potential influencing variables may also be counterbalanced or randomized. In some cases, a mixed design may be used, which is a combination of an independent groups design and a repeated measures design.

In fact, there seems to be broad consensus among social scientists that causal inferences cannot be made simply and directly from empirical data, regardless of whether they are obtained from randomized experiments, systematic observations, or advanced statistical models. Thus, there are methodologists (e.g., Lieberson
1985) who apparently consider it as a myth that causality can be determined exclusively by randomization. Rather, causal inferences have to be based on sound theories and hypotheses. Cox (1990) argues that a major limitation of experimental designs and the randomization principle is often the lack of a specific theory of the underlying processes to be explained, such as learning or problem solving. However, it should be such a theory that must "guide" both the design of data collection and the way data are analyzed and interpreted. Theoretical arguments have to specify the particular regularities or even mechanisms for how a particular event or treatment (i.e., the cause) consistently produces an effect in time.

The art of experimental design is to choose the control conditions that provide information about essential features in an efficient way. The planning of research involves manifold decisions with regard to the design of investigation. Clearly, the identification of causation presupposes necessarily to work with one or more control groups (e.g., the Solomon four-group design) so that comparisons can be made between the effects of the treatment. A simple control group design may be sufficient in many cases to detect specific effects of a treatment, but often additional groups are essential to identify the aspects of the treatment under control of extraneous variables that are threats to internal and external validity.

Referring to the field of animal learning, but certainly generalizable onto the field of human learning, Church (2003) has discussed some important issues of research designs centering on the questions how research on learning should be conducted, how empirical data could be analyzed and explained. Actually, with regard to the conduction of research on learning there are some common problems for all research designs. First of all, it must be determined how many subjects must be assigned to the groups, how many trials in the learning process are included and how errors should be handled. In both between-group and within-group designs, the number of necessary participants should be assessed on the basis of a power analysis, which refers to an estimate of the within-group variability to be expected and an estimate of the expected size of the treatment effect (Wilcox 2003). The number of subjects in repeated measures designs is basis on similar considerations. In the single-subject designs, however, the decisions are based on 
convention. Concerning the number of trials, Church (2003) argues that researchers typically choose such a number of trials sufficient to produce complete learning. This number may be chosen before the beginning of the experiment based on the number used in similar experiments, or it may be based on some formal criterion of stability.

The problems of data analysis in animal learning research are similar to those in other psychological research. The major role of inferential data analysis in research in animal and human learning is to provide a consistent standard for conclusions based on quantitative data. It also provides a succinct report of the conclusions regarding significance, confidence intervals, and magnitudes of results. Without the conventions of inferential statistics, different researchers might reach different conclusions from the same results. The key intuition is that a result is significant if it is consistently observed. Finally, Church (2003) discusses the several ways to explain (animal) learning. They include a description of the controlling variables, statements of the principles, and process models based on psychological, biological, or mathematical variables. Church emphasizes that results of investigations are facts that can be observed whereas the explanations are alternative ways to understand these results. A good explanation will provide a succinct and accurate description of a wide range of results.

The essence of experimental research on learning is the detection of functional relationships between input and output variables. In learning experiments, the input variable is some treatment condition, and the output variable is some measure of overt behavior. The central idea of quantitative research is that both variables can be expressed on a quantitative scale so that it is possible to describe a functional (linear) relationship between the input and the output. In terms of Church (2003), a functional explanation will be more general (such that it applies to several variables), more analytical (such that it will suggest causality), and more quantitative (such that it will provide a mathematical function).

Functional relationships between input and output variables are especially important in process models of learning that involve also intervening variables, which may be psychological, biological, or mathematical. In process models of learning, the relationships between the treatment (input variable) and at least one intervening variable must be specified as well as the relationship between at least one intervening variable and the output must be specified. Clearly, there may be more intervening variables with relationships which must be specified. The history of research on learning shows the importance of psychological and biological intervening variables (such as perception, attention, memory, and their physiological correlates). However, intervening variables can also be described in mathematical terms so that stochastic models of learning result. Process models of learning can be specified simultaneously in psychological, biological, and mathematical terms. This provides a way to relate the psychological and biological bases of learning and to specify them in terms of mathematical functions.

\section{Cross-References}

- Bayesian Learning

- Design Experiments

- Mathematical Models of Learning

- Methodologies of Research on Learning

- Mixed-Methods Research

\section{References}

Bracht, G. H., \& Glass, G. V. (1968). The external validity of experiments. American Educational Research Journal, 5(4), 437-474.

Campbell, D. T., \& Stanley, J. C. (1963). Experimental and quasiexperimental designs for research on teaching. In N. L. Gage (Ed.), Handbook of research on teaching (pp. 171-246). Chicago: Rand McNally.

Church, R. (2003). Animal learning. In I. B. Weiner, D. K. Freedheim, J. A. Schinka, \& W. F. Velicer (Eds.), Handbook of psychology (Research methods in psychology, Vol. 2, pp. 271-288). Huboken, NJ: Wiley.

Cook, T. D., \& Campbell, D. T. (1979). Quasi-experimentation: Design and analysis issues for field settings. Chicago: Rand MacNally.

Cox, D. R. (1990). Role of models in statistical analysis. Statistical Science, 5, 169-174.

Creswell, J. W. (2005). Educational research. Planning, conducting, and evaluating quantitative and qualitative research (2nd ed.). Upper Saddle River, NJ: Pearson.

Fisher, R. A. (1925). Statistical methods for research workers. Edinburgh: Oliver \& Boyd.

Kirk, R. E. (2003). Experimental design. In I. B. Weiner, D. K. Freedheim, J. A. Schinka, \& W. F. Velicer (Eds.), Handbook of psychology (Research methods in psychology, Vol. 2, pp. 3-32). Huboken, NJ: Wiley.

Lieberson, S. (1985). Making it count. Berkeley, CA: University of California Press.

Shadish, W. R., Cook, T. D., \& Campbell, D. T. (2002). Experimental and quasi-experimental designs for generalised causal inference. New York: Houghton Mifflin Company. 
Wilcox, R. D. (2003). Power: Basics, practical problems, and possible solution. In I. B. Weiner, D. K. Freedheim, J. A. Schinka, \& W. F. Velicer (Eds.), Handbook of psychology (Research methods in psychology, Vol. 2, pp. 65-86). Huboken, NJ: Wiley.

\section{Experimental Classrooms}

High Performance Learning Spaces

\section{Experimental Learning Environments}

\author{
ElKe Sumfleth $^{1}$, Maik Walpuski ${ }^{2}$ \\ ${ }^{1}$ University of Duisburg-Essen, Essen, Germany \\ ${ }^{2}$ Leuphana University of Lüneburg, Lüneburg, \\ Germany
}

\section{Synonyms}

Inquiry environments; Inquiry learning

\section{Definition}

Learning environments are defined as places where individuals can learn by using different resources, such as media, tools, and laboratory equipment. An experimental learning environment has necessarily to be distinguished from an experiential learning environment which is defined very generally as making experiences. For the term experiment different definitions can be found. One very simple definition is that an experiment means to manipulate equipment and/or variables purposefully. In a scientific experiment, the aim is usually to gain new knowledge or to approve existing knowledge by researching the influence of different variables. For this reason, the variables have to be changed systematically while other variables are controlled. An explorative (or inductive) design, generating new ideas or hypotheses and a deductive design, being derived from an existing theory can be distinguished. The deductive design is often described as the scientific method. This means that an observation is explained or a prediction is made based on already existing theories. In the next step, the prediction or explanation (=hypothesis) is tested by manipulation and controlling certain variables. In a last step, the hypothesis is either supported by the result or rejected. Although it can be argued that scientific knowledge can be acquired in many different ways and that some important scientific discoveries have only been made by chance, for a learning environment this discussion is only of low importance because the structured experimentation is a learning goal in itself.

Accordingly, an experimental learning environment can be defined as a place where individuals can learn by generating or testing hypotheses in a controlled way.

\section{Theoretical Background}

Experimental learning environments play a decisive role especially in the field of science education. The educational standards of different nations explicitly describe requirements focusing on the acquirement of knowledge by means of experiments. The American science standards and benchmarks regard scientific inquiry as a subcategory of nature of science and differentiate between "evidence and reasoning in inquiry," "scientific investigations," "scientific theories" and “avoiding confirmation bias." Zimmerman (2005, p.

6) describes numerous procedural and conceptual activities in this whole field: "Scientific investigations broadly defined includes numerous procedural and conceptual activities such as asking questions, hypothesizing, designing experiments, making predictions, using apparatus, observing, measuring, being concerned with accuracy, precision and error, recording and interpreting data, consulting data records, evaluating evidence, verification, reacting to contradictions or anomalous data, presenting and assessing arguments, constructing explanations (to self and others), coordinating theory and evidence, performing statistical calculations, making inferences and formulating and revising theories and models." Students apparently do not learn scientific working and reasoning by simply practicing it, but have to be explicitly taught in the requested skills (Schwartz et al. 2004).

The procedural approach chosen to conduct a scientific investigation is influenced by two different kinds of aims. The first aim is to generate an effect, which is often used by engineers or in everyday life situations. The second - more scientific - aim is to test a hypothesis (Schauble 1996). While testing hypotheses, different strategies are used. It is remarkable that 
variables expected to yield a positive effect are more frequently investigated than those expected to have a negative effect. As a result, the experiments are often only adequate to confirm a hypothesis ("confirmation bias") but not to reject it. The variable that is assumed to cause the positive effect is kept constant in order to maintain the effect (HOTAT strategy - Hold One Thing At a Time). Zimmerman and Glaser (2001) argue that people tend to verify the hypothesis' general validity instead of verifying the hypothesis itself. In contrast, an expected negative outcome or a negatively formulated hypothesis caused more than $50 \%$ of all children and adults to use a systematical approach in which only one thing is varied at a time in order to avoid the negative outcome (VOTAT strategy - Vary One Thing At a Time) (Zimmerman and Glaser 2001; Zimmerman 2005). Especially younger children vary more than one variable or all variables at a time in order to avoid a negative effect (CA strategy - Change All).

Although experiments are a somewhat natural part of science instruction, they are often used inadequately and structured like a "recipe" by narrow prescriptions. Learners are given very little opportunity to discuss or test their own hypotheses. As a result, experiments often do not take account of common mis- and preconceptions and students remain unable to construct the accepted scientific understanding on their own. Lunetta (1998, p. 250) states that "to many students, a 'lab' means manipulating equipment and not manipulating ideas." Students' attempts in testing hypotheses are often deficient believing that hypotheses can only be adequately tested by verification (Lederman 1992). They have difficulties in distinguishing between hypotheses, laws, and theories and in deducing results from experiments (Lederman et al. 2002). They tend to accept scientific theories as the absolute truth. As a consequence, it is necessary that students learn about typical scientific methods, that is, how to carry out experimental investigations. Therefore, an instructional approach based on experiments has to consider generating hypotheses, testing these systematically in experiments, and integrating the new information thus obtained into students' knowledge structures. Although there are reasonable doubts about the existence of the scientific method (Lederman et al. 2002) as a stepwise procedure that all scientists follow, there are some typical steps which are helpful for organizing scientific inquiry for novices. Klahr (2000) describes a very detailed model of "scientific discovery as dual search" (SDDS) dividing the scientific work into three main areas: searching in the hypothesis space, testing hypothesis, and evaluating evidence. He differs between a hypothesis space and an experiment space. In the hypothesis space, learners' knowledge about the relations between variables and the effects of any changes in these variables is presented. Here the hypotheses are formulated, modified, evaluated, and stored. The experiment space contains all the tests to prove a hypothesis. In this context, applying scientific knowledge means systematically selecting, planning, and conducting experiments. The evaluation of evidence requires a comparison between the hypothesis and the outcome. This ends either in accepting, rejecting, or modifying a theory or hypothesis. Klahr and Dunbar consider the whole process as "problem solving that is characterized as a guided search and information-gathering task" (Zimmerman 2005, p. 9). This SDDS model describes successful strategies for discovering or producing new information, but it does not allow to draw conclusions on the integration of this information into the existing knowledge structure, which is quite important for describing the learning process. Table 1 summarizes the main statements.

Recent research focuses improve the knowledge about scientific investigations. Important factors with regard to experimental learning environments are

- The learner's idea of an experiment's aim, his or her conceptual domain-specific pre-knowledge, and developmental psychological aspects

- The number of variables to be considered and practice, instruction, feedback, and structuring

- Fit of the outcome of an experiment and a person's theory or hypothesis

\section{Important Scientific Research and Open Questions}

Experimental learning environments aim at least at two different goals, at an improvement in conceptual knowledge often understood as content knowledge and a development of procedural knowledge focusing on acquirement of knowledge strongly related to the use of experiments and models and combined with the realization of experiments in lab situations. 
Experimental Learning Environments. Table 1 Describing SDDS model

\begin{tabular}{|c|c|c|c|}
\hline Phase & Aims and functions & Deficiencies & Criteria for successful experimentation \\
\hline Generally & $\begin{array}{l}\text { - Acquirement of knowledge } \\
\text { by means of testing of } \\
\text { hypotheses by experiments }\end{array}$ & $\begin{array}{l}\text { - Predominance of } \\
\text { affirmation by } \\
\text { experiments }\end{array}$ & $\begin{array}{l}\text { - Testing of hypotheses by experiments } \\
\text { - Daily-life context } \\
\text { - Embedding of experiments in a problem- } \\
\text { solving and stimulating instructional course }\end{array}$ \\
\hline Planning & $\begin{array}{l}\text { - Identifying a problem } \\
\text { - Analyzing a problem } \\
\text { - Formulating hypotheses } \\
\text { - Differentiation between } \\
\text { control and test variables } \\
\text { - Planning the conducting of } \\
\text { the experiment }\end{array}$ & $\begin{array}{l}\text { - Aim and meaning } \\
\text { of the experiments are } \\
\text { not identified } \\
\text { - Difficulties in } \\
\text { formulating } \\
\text { hypotheses } \\
\text { - Non-systematic use } \\
\text { of control and test } \\
\text { variables }\end{array}$ & $\begin{array}{l}\text { - Self-determined planning and generating of } \\
\text { hypotheses support by a structured } \\
\text { (structured) training and structuring aids }\end{array}$ \\
\hline $\begin{array}{l}\text { Conducting } \\
\text { experiments }\end{array}$ & $\begin{array}{l}\text { - Acquiring experimental skills } \\
\text { - Recording observations }\end{array}$ & $\begin{array}{l}\text { - Mistakes in } \\
\text { observation and } \\
\text { protocols }\end{array}$ & $\begin{array}{l}\text { - Self-determined experimentation (in small } \\
\text { groups) } \\
\text { - Feedback and responsive teacher } \\
\text { intervention } \\
\text { - Significant effect } \\
\text { - Successful experimentation }\end{array}$ \\
\hline $\begin{array}{l}\text { Evaluating } \\
\text { results }\end{array}$ & $\begin{array}{l}\text { - Data clearing } \\
\text { - Explaining observations } \\
\text { - Working on relations and } \\
\text { crosslinks } \\
\text { - Summarizing and presenting } \\
\text { results } \\
\text { - Constructing references to } \\
\text { the hypotheses } \\
\text { - Developing generalizing } \\
\text { statements } \\
\text { - Relation between theory and } \\
\text { practice } \\
\text { - Acquirement of knowledge }\end{array}$ & $\begin{array}{l}\text { - Illogical conclusions } \\
\text { - Unproved } \\
\text { causalities } \\
\text { - Difficulties in } \\
\text { referring to the } \\
\text { hypotheses } \\
\text { - Insuperable beliefs }\end{array}$ & $\begin{array}{l}\text { - Autonomous evaluation and discussion in } \\
\text { small groups } \\
\text { - Control of learning aims and outcome } \\
\text { - Organizing learning opportunities }\end{array}$ \\
\hline
\end{tabular}

With regard to current research and open questions in the field of experimental learning environments different areas have to be differentiated:

- Achievement of conceptual knowledge by experiments

- Achievement of procedural knowledge about the use of experiments

- Learning to do practical work

- Quality of instruction in classroom situations with regard to the use of experiments

In science education, the main question concerns the degree of openness in experimental learning situations with regard to learning achievement. "Cook book recipes" are as ineffective as totally open experimental tasks. The use of interaction kits (Walpuski et al. 2008) combines the advantages of open learning situations with a structured learning environment. The whole learning material, the laboratory equipment required and some instructional hints, for one group of students is arranged in one box including the problem to be solved by the group. The core of the learning environment is a special kind of collaborative group work called group investigation (Sharan and HertzLazarowitz 1980). The goal of the group discussion is the joint construction of a problem-solving strategy. The students have to select the required instruments and to combine them in an experimental setting. 
This learning environment has an explorative aim intending the development of hypotheses, the development and conduction of experiments to test the hypotheses, and an evaluation of the experiment including a reflection of the hypotheses. Additionally, the equipment allows the realization of different problem-solving approaches. Known preconceptions might be disproved by suitable experiments.

Comparing the learning outcome of this collaborative learning through complex and open-ended experimental inquiry tasks with direct instruction by the teacher in front of the class shows significant effects in advantage of the collaborative group work with a slightly higher increase in learning (Sumfleth et al. 2004). The effect sizes are small as expected when the important variables such as students' pre-knowledge, difficulty of problem formulation, time on task and teacher's personality are controlled. The differences between both groups increase from the pre-post to the prefollow-up test comparison because of a greater decrease of achievement in the classes without group investigation. These results show that experimental learning environments with small groups can be at least as effective as direct instruction even if instruction time is kept constant. However, the video data of both kinds of instruction produce evidence for the following conclusions: While the teacher in the control group collects the relevant ideas from the students and corrects mistakes during experimentation - the direct instruction is carried out at its best - the students of the intervention group are often unable to identify and correct their own mistakes while working alone. In group work situations the students often forget the ideas they want to test, they describe observations of experiments and consider them to be conclusions, and they do not relate their results to the ideas or hypotheses from the beginning.

The use of feedback has a verifiable positive effect on students' achievement in small work groups, when the feedback helps to avoid correct ideas being wrongly disapproved because of operational mistakes (Walpuski et al. 2008). An example-based metacognitive structuring training can offer students a particular introduction to the appropriate use of structuring aids in order to support self-regulated scientific inquiry. Structuring measures consist of ideacards to put down every idea coming up to solve the experimental problem and experiment-cards to write down the description of every experiment conducted and the depending conclusions. This process helps to ensure that the students do not forget to review their ideas and to draw conclusions on every experiment. In addition they are supported by flow-charts developed during the training, which represent an "optimal" way for conducting scientific studies and thus acquiring scientific knowledge. The success of this intervention could be evidenced by the results of a content-based test as well as a structuring test. Qualitative analyses of the video data show a lower number of omission mistakes and fitting mistakes for the groups which obtained the structuring training.

A further study (Schmidt-Weigand et al. 2009) proves the usefulness of additional support by means of stepwise structuring facilities. Comparing different supporting means in problem-solving situations, a worked example given as a whole, a worked example presented incrementally, and a worked example presented incrementally with additional strategic prompts, leads to the finding that students selfregulation results in an improved learning outcome when each solution step is prompted by a hint (e.g., note taking, sketching, etc.). The experience of competence seems to be higher and the cognitive load lower.

\section{Cross-References}

- Design of Learning Environments

- Group Learning

- Interactive Learning Environments

- Learning Environments

- Learning in Information-Rich Environments

- Remote Laboratory Experiments in Virtual Immersive Learning Environments

- Technology-Based Learning Environments for Science Inquiry

\section{References}

Klahr, D. (2000). Exploring science. The cognition and development of discovery processes. Massachusetts: Institute of Technology.

Lederman, N. G. (1992). Students' and teachers' conceptions of the nature of science: A review of the research. Journal of Research in Science Teaching, 29, 331-359.

Lederman, N. G., Abd-El-Khalick, F., Bell, R. L., \& Schwartz, R. S. (2002). Views of nature of science questionnaire: Toward valid and meaningful assessment of learners' conceptions of nature of science. Journal of Research in Science Teaching, 39, 497-521.

Lunetta, V. N. (1998). The school science laboratory: Historical perspectives on contexts for contemporary teaching. In B. J. Fraser \& K. G. Tobin (Eds.), International handbook of science education. Part one (pp. 249-262). Dordrecht: Kluwer. 
Sharan, S. \& Hertz-Lazarowitz, R. (1980). A group investigation method of cooperative learning in the classroom. In S. Sharan, P. Hare, C. Webb \& R. Hertz-Lazarowitz (Eds.), Cooperation in education (pp.14-46). Prove: Brigham Young University Press.

Schauble, L. (1996). The development of scientific reasoning in knowledge-rich context. Developmental Psychology, 32, 102-119.

Schmidt-Weigand, F., Hänze, M., \& Wodzinski, R. (2009). Complex problem solving and worked examples. Zeitschrift für Pädagogische Psychologie, 23(2), 129-138.

Schwartz, R. S., Lederman, N. G., \& Crawford, B. A. (2004). Developing views of nature of science in an authentic context: An explicit approach to bridging the gap between nature of science and scientific inquiry. Science Education, 88, 610-645.

Sumfleth, E., Nicolai, N., \& Rumann, S. (2004). Schulische und häusliche Kooperation im Chemieanfangsunterricht [Academic and domestic cooperation in chemistry beginners' lessons.]. In J. Doll \& M. Prenzel (Eds.), Bildungsqualität von Schule: Lehrerprofessionalisierung, Unterrichtsentwicklung und Schülerförderung als Strategien der Qualitätsverbesserung. [Quality of school education: Teachers' professionalization, development of tuition and encouragement of pupils as strategies for a quality improvement.] (pp. 284-302). Münster: Waxmann.

Walpuski, M., Wahser, I., \& Sumfleth, E. (2008). Improvement of inquiry-learning using collaborative tasks. In B. Ralle \& I. Eilks (Eds.), Promoting successful science education - The worth of science education research (pp. 197-201). Shaker: Aachen.

Zimmerman, C. (2005). The development of scientific reasoning skills: What psychologists contribute to an understanding of elementary science learning. Final Draft of a Report to the National research Council. Retrieved June 13, 2011, from http://www7.nationalacademies.org/bose/Corinne_Zimmerman_Final_Paper.pdf

Zimmerman, C., \& Glaser, R. (2001). Testing positive versus negative claims: A prelimanary investigation of the role of cover story in the assessment of experimental design skills. (Tech. Rep. No. 554). Los Angeles: UCLA National Center for Research on Evaluation, Standards, and Student Testing.

\section{Experimentation}

The process of actively and intentionally varying the performance of a known action or behavioral sequence in order to discover new variations which may yield interesting results. This activity is driven by an intrinsic motivation to discover new things, and broadens the learner's repertoire, thus improving the ability to solve future problems.

\section{Experimenting}

- Innovation and Learning Facilitated by Play

\section{Expert Cognitive Modeling and Problem-Based Learning}

\author{
Susan Pedersen \\ Department of Educational Psychology, Texas A\&M \\ University, College Station, TX, USA
}

\section{Synonyms}

Cognitive apprenticeship modeling

\section{Definition}

Expert cognitive modeling is an instructional approach that can help learners develop a mental model of the types of domain knowledge and strategies that an expert would bring to bear in addressing a complex task. Typically, this is accomplished by having the expert think aloud while engaging in a task. Learners are able to observe not only the physical performance involved, but the internal cognitive processes that guide the actions of the expert. Because normally hidden cognitive processes are made overt, the learner has access to how specific strategies are useful within the context of an immediate problem or task.

Models are by their nature incomplete representations of a phenomenon; modeling refers to the act of building that representation. Confusingly, the term modeling can be used to describe the activity of both the expert and the novice. As the expert thinks aloud, he or she models whatever knowledge and strategies seem most relevant, but does not attempt to consider every single fact or heuristic that may influence his or her actions. Likewise, the learner models the expert process through observation, but that model does not include everything that may have influenced the expert's process. In both cases, modeling aids in bringing novice practice closer to that of the expert.

\section{Theoretical Background}

The role of modeling in learning was a focus of social learning theory (Bandura 1971). Research based on SLT showed that learners who observed the behavior of others in a learning process, even though they did not participate in that process themselves, were often found later to apply newly acquired behaviors to other tasks. The cognitive apprenticeship model proposed by 
Collins et al. (1989) extended the idea of modeling beyond behaviors to focus on the cognitive processes underlying those behaviors. They argued that the instructional approaches typically used in schools render key aspects of expert practice invisible to learners; a cognitive apprenticeship approach makes the strategies underlying expert practice explicit. Methods useful in a cognitive apprenticeship approach include cognitive modeling, coaching, and scaffolding. These methods allow novices to observe and compare their cognitions with those of the expert in order to develop and apply the same knowledge and skills as the expert.

Cognitive modeling has the potential to enhance learning through instructional approaches that engage learners in complex tasks, such as problem-based learning $(\mathrm{PBL}) . \mathrm{PBL}$ is an approach to instruction that uses problems as the stimulus and focus for learner activity. Instruction starts with the presentation of a problematic situation, and learning occurs as a direct result of learners' efforts to define and solve the problem. As learners reason their way through the complexities posed by the problem, they have the opportunity to develop the same kinds of strategies and habits of mind that experts use. However, PBL requires learners to be, at least to some degree, self-directed. Yet, even in medical programs, learners initially have difficulty with the lack of externally imposed structure and responsibility for self-directed study (Williams 1993). Young learners have even less developed planning skills and are less able to reflect upon their efforts and change them when necessary. Giving learners access to both the actions an expert would take and the thinking that guides those actions can help learners to structure their activity and consider useful strategies they can apply. Such modeling can be delivered by a teacher or tutor in those situations where one is available, but one promising use of technology lies in the possibility of giving learners access to expert cognitive modeling in a justin-time fashion. This can be accomplished by connecting learners to experts as they engage in PBL, or through the use of videos of experts thinking aloud about their process as they engage in various tasks useful in developing a solution to the same or a similar problem. In both cases, the modeling occurs when individual learners ask for it, not in a whole-class, externally imposed manner.

Pairing PBL and expert cognitive modeling is supported by multiple theoretical perspectives. From a cognitive perspective, learners create mental models of relevant phenomena as they wrestle with the problem posed in a PBL unit. These mental models result from the internalization of external representations. As such, modeling an expert's process (an external representation) in PBL engages learners in an iterative process (internalization) of model building and revision, challenging their initial ideas and allowing them to flesh out their model of these phenomena. More recently, research on PBL has been anchored in the situative perspective, where learning can be viewed as improved participation in the activity of a community (Sfard 1998; Cobb and Bowers 1999). This perspective can be seen as the result of what has been termed the "second wave" of the cognitive revolution, which developed in reaction to the lack of emphasis on context in information processing theory (the first wave). From this perspective, $\mathrm{PBL}$ creates a context in which learners participate with peers in developing solutions to problems valued within the wider community. Gradually, the learner constructs his or her understanding of the knowledge useful to the community and adopts its language and customs. Because members of the community actually depend on the successful participation of other members, collaboration rather than competition is fostered. However, a limitation of PBL as it is typically implemented is that learners work in teams with peers who have no greater knowledge of the domain than they do. Different team members may have different types of prior knowledge, but there is no one in the group who can be considered an expert within the domain. Yet communities are typically able to draw upon different types of expertise, so access to expert processes actually brings PBL into closer alignment with the authentic practices of a community. Offering expert cognitive modeling during PBL can help learners develop a deeper understanding of how the community addresses similar problems. This approach has been shown to enhance the quality of both learners' processes and the solutions they develop during PBL (Pedersen and Liu 2002).

\section{Important Scientific Research and Open Questions}

Evidence exists supporting the effectiveness of cognitive apprenticeship style instructional methods in general (Collins et al. 1989) and for the incorporation of 
expert cognitive modeling during PBL specifically (Pedersen and Liu 2002). However, much research remains to be done to build a knowledge base of how to design and offer expert cognitive modeling during PBL or other complex instructional approaches. Three areas deserve particular attention. First, how does expert cognitive modeling affect group dynamics during PBL? PBL is usually conducted with teams of learners working collaboratively. One of the most powerful effects of expert cognitive modeling may lie in its potential to impact a few learners, who then impact the performance of the entire group. Examining the impact of expert cognitive modeling on the functioning of the group may provide some insights into how these groups function like a scientific community to affect the understandings and performances of individuals. Second, do learners build more robust models of expert thinking when they have access to multiple experts who offer different perspectives or approaches? Or does this confuse the issue, perhaps creating a cognitive overload, by forcing learners to evaluate strategies that they may not fully understand? Finally, what are the strengths and limitations of technology in providing learners with access to expert cognitive modeling? Videos of experts can be developed by teams of subjectmatter experts and instructional designers, leading to high quality modeling. In fact, in some cases, the quality might be superior to that which teachers can offer, in that teachers may not all possess the expertise necessary or may focus their modeling on their actions rather than the reasons for those actions. Learners can have access in a just-in-time manner, as they find a need for such support, and they can review it as many times as necessary. However, live experts may be able to better evaluate the needs of learners and therefore provide more appropriate models. Learners can also probe expert thinking through questions and requests for clarification. Understanding how to effectively leverage the potential of technology to provide learners access to expert models of strategies useful in addressing complex tasks may make approaches like PBL more viable for a wider range of learners.

\section{Cross-References}

- Cognitive Apprenticeship Learning

- Model-Based Reasoning

- Problem-Based Learning

\section{References}

Bandura, A. (1971). Social learning theory. New York: General Learning Press.

Cobb, P., \& Bowers, J. (1999). Cognitive and situated learning perspectives in theory and practice. Educational Researcher, 28(2), 4-15.

Collins, A., Brown, J. S., \& Newman, S. E. (1989). Cognitive apprenticeship: Teaching the crafts of reading, writing, and mathematics. In L. B. Resnick (Ed.), Knowing, learning and instruction: Essays in honor of Robert Glaser. Hillsdale: Lawrence Erlbaum Associates.

Pedersen, S., \& Liu, M. (2002). The effects of modeling expert cognitive strategies during problem-based learning. Journal of Educational Computing Research, 26(4), 353-380.

Sfard, A. (1998). On two metaphors for learning and the dangers of choosing just one. Educational Researcher, 27(2), 4-13.

Williams, S. M. (1993). Putting case based learning into context: Examples from legal, business, and medical education. Journal of the Learning Sciences, 2, 367-427.

\section{Expert Perceptual and Decision- Making Skills: Effects of Structured Activities and Play}

\author{
Bruce Abernethy ${ }^{1}$, JeAn Côté ${ }^{2}$ \\ ${ }^{1}$ School of Human Movement Studies, The University \\ of Queensland, Brisbane, Australia \\ ${ }^{2}$ School of Kinesiology and Health Studies, Queen's \\ University, Kingston, ON, Canada
}

\section{Synonyms}

Developmental and practice histories; Practice and play in developing expertise

\section{Definition}

Experts are those individuals who consistently display exceptional levels of performance in a particular domain. These exceptional levels of performance are, by definition, attained by only a limited number of individuals and are well beyond that achievable by people less skilled and less practiced in the field of specialization. The perceptual skills of experts are those skills that enable the experts to quickly and accurately interpret the current and future state of the performance environment in a manner that facilitates effective responding. The key perceptual skills of experts include the capability to rapidly encode, 
recognize, and recall domain-specific patterns and to predict (anticipate) future events within the domain on the basis of information available early within an event sequence. The decision-making skills of experts are those skills that enable the experts to quickly select the most appropriate response (or course of action) for any given situation from amongst a range of possible options. Expert decision making is dependent upon accurate synthesis and analysis of not only current perceptual information but also stored information in relation to contextual and event probabilities accumulated from past experiences within the domain. Different types of learning experiences throughout the lives of experts may contribute in different ways to their development of expertise. Structured activities are those practice activities undertaken, generally under the supervision of an adult or coach, in which the specific intention is to facilitate skill development through repetition, progressive challenge, and error reduction. In contrast, play activities are those activities undertaken for their intrinsic interest and enjoyment, usually in the absence of adults, in the absence of rules and structure, and in the absence of the explicit, instrumental goal of skill improvement. The question of interest is to what degree do structured practice activities and (unstructured) play each contribute to the development of the perceptual and decision-making skills characteristic of experts?

\section{Theoretical Background}

Across the full spectrum of the domains of human endeavors (business, sports, music and arts, academia, etc.), expertise is highly valued and sought. For this reason, knowing the component skills that differentiate the expert from the nonexpert and, in turn, understanding how the expert advantage on these skills has developed, is important. In activities that are time constrained and the performance environment is variable and, in part, unpredictable - activities like ball sports or driving a motor vehicle - experts consistently outperform nonexperts on pattern recall and anticipation tasks derived from their domain of specialization. Expert basketball players, for example, when briefly shown unfamiliar video of basketball games are better able to recall the positions of players on the court at a given instant than are less-skilled players (although this advantage largely disappears if the presented information shows players in completely random formations). Expert drivers, shown video of the forward display from a moving vehicle, are better able to predict future hazard situations on the road than are inexperienced drivers. These differences in perceptual skill between experts and nonexperts may be apparent even under situations where either the basic visual capabilities (as measured by acuity, for instance) or the eye movement patterns of the different skill groups are indistinguishable. This indicates that it is not the ability to detect information but rather the ability to interpret and use the available information that is the limiting factor to the perceptual performance of the nonexpert. Comparable expert advantages are also evident in tasks that require decision making. When faced with a scenario from their domain of specialization that requires selecting the most appropriate option from a range of possible response choices, experts are consistently found to respond not only more accurately (selecting the correct response more frequently) but also more rapidly; in so doing, minimizing the speedaccuracy trade-offs typically observed in such situations.

Given that the nature of the expert advantage in perception and decision making can be determined, the logical consequential question is how do such differences develop? Setting aside the possibility of expertnonexpert differences in the heritability of genes advantageous to these tasks, a likely source of the expert advantage is favorable learning experiences during development. Examination of this question has been heavily influenced by the work of Benjamin Bloom (1985) who pioneered the retrospective examination of the practice and developmental histories of experts. Anders Ericsson and colleagues (e.g., Ericsson et al. 1993) have argued that the key determinant of the development of expertise is the amount of deliberate practice that an individual has accumulated, with experts across the domains of music and chess requiring some 10 years and $10,000 \mathrm{~h}$ of deliberate practice to acquire expertise. Deliberate practice entails those structured activities undertaken with the explicit goal of improving performance and requiring significant and prolonged investment of effort (mental, physical, or both). Such practice is frequently "worklike," solo (in the case of the musicians) and not necessarily enjoyable. In contrast, Côté and colleagues 
(e.g., Côté et al. 2003) have argued that the type of play activities undertaken during early childhood and up to adolescence may be equally important in developing expertise and sustaining motivation, especially as manifest within the sports domain. The term deliberate play has been coined to refer to activities such as backyard football games and street hockey where the principal goal for participation is enjoyment and the activities are characterized by an abundance of imitation and a lack of rigid structure and rules. Deliberate practice is generally adult devised and supervised whereas engagement in deliberate play typically occurs without adult involvement.

\section{Important Scientific Research and Open Questions}

Direct examination of the significance of structured (deliberate practice) activities vs. unstructured (deliberate play) activities in the development of expert perception and decision making has been most evident within the sports domain. Studies of the development of expertise in sport that have adopted a deliberate practice framework (e.g., Hodges and Starkes 1996) have demonstrated that there is a monotonic relationship between the amount of deliberate practice and performance in sports just as in other domains. (In other words, as the amount of accumulated deliberate practice increases, performance also systematically increases.) However, unlike the situation in music for instance, group/team practice often appears equally important as solitary practice, enjoyment is frequently positively related to engagement in practice, and many instances can be documented of world-class performers whose accumulated quantum of deliberate practice is substantially less than $10,000 \mathrm{~h}$ or 10 years. A growing body of evidence suggests that the extent of engagement in deliberate play may be an equally important determinant/predictor of the subsequent emergence of expertise. For example, Soberlak and Côté (2003) found that up to age 20, elite ice hockey players had spent just as much time in deliberate play activities as in deliberate practice. Berry et al. (2008), in a study of elite Australian football players, found that both the amount of time invested in structured practice activities in invasion-type sports and the amount of time spent in deliberate play of the same types of sports were significantly greater for players classifed by coaches as exceptional decision makers compared to ones classified as poor decision makers. The amount of experience accumulated in the playing of invasion-type sports activities rather than the specific intent of the activity (play or practice) appeared to be the most crucial factor necessary for the eventual emergence of expert perceptual and decision-making skill.

Research is still in its infancy on the impact of the practice and play experienced during the developing years on the subsequent acquisition of expertise. The ongoing challenge for researchers is to go beyond the simple analysis of the quantum of hours devoted to each type of activity to understanding the microstructure of these activities and to an understanding of how (and through what processes) different types of activity engage the learner. The evidence available to date suggests that the type of practice and play conducive to the development of expertise may vary between, and possibly also within, domains but it remains to be seen whether such variability in findings will ultimately thwart the development of models and theories of the development of expertise that are able to be generalized across domains. An important practical consideration that arises from examination of the practice and play antecedents of expertise is the issue of whether enhanced development of expertise is best facilitated through early or late specialization. While early specialization may possibly represent the best path to success in activities, like gymnastics, where peak performance is achieved prematurely, early specialization may also have undesirable consequences with respect to levels of enjoyment and lifetime engagement in physical activity. Multiple pathways are possible to expertise in other activities, and indeed engagement in high amounts of deliberate play during the preadolescent years may not only help sustain a person's commitment to physical activity but may also be functionally advantageous for perceptual and decision-making skill development in many team ball sports (see Côté et al. 2007).

\section{Cross-References}

- Adaptability and Learning

- Adaptation and Anticipation: Learning from Experience

- Anticipation and Learning

- Choice Reaction Time and Learning

- Cognitive Skill Acquisition 
- Complex Skill Acquisition

- Deliberate Practice and Its Role in Expertise Development

- Development of Expertise

- Expert Learner

- Human Cognition and Learning

- Learning at Play

- Learning by Experience

- Perceptual Learning

- Play and Its Role in Learning

- Play, Exploration, and Learning

- Skill Acquisition/Learning

\section{References}

Berry, J., Abernethy, B., \& Côté, J. (2008). The contribution of structured activity and deliberate play to the development of expert perceptual and decision-making skill. Journal of Sport \& Exercise Psychology, 30, 685-708.

Bloom, B. S. (Ed.). (1985). Developing talent in young people. New York: Ballantine Books.

Côté, J., Baker, J., \& Abernethy, B. (2003). From play to practice: A developmental framework for the acquisition of expertise in team sport. In J. Starkes \& K. A. Ericsson (Eds.), Expert performance in sports: Advances in research on sport expertise (pp. 89-110). Champaign: Human Kinetics.

Côté, J., Baker, J., \& Abernethy, B. (2007). Practice and play in the development of sport expertise. In G. Tenenbaum \& R. C. Eklund (Eds.), Handbook of Sport Psychology (3rd ed.) (pp. 184-202). Hoboken, NJ: Wiley.

Ericsson, K. A., Krampe, R. T., \& Tesch-Römer, C. (1993). The role of deliberate practice in the acquisition of expert performance. Psychological Review, 100, 363-406.

Hodges, N. J., \& Starkes, J. L. (1996). Wrestling with the nature of expertise: A sport-specific test of Ericsson, Krampe and TeschRömer's (1993) theory of deliberate practice. International Journal of Sport Psychology, 27, 400-424.

Soberlak, P., \& Côté, J. (2003). The developmental activities of elite ice hockey players. Journal of Applied Sport Psychology, 15, 41-49.

\section{Expert Performance}

Expertise

\section{Expert System}

- Adaptive Instruction Systems and Learning

- Ontology and Semantic Web

\section{Expertise}

TAmara van Gog

Institute of Psychology, Erasmus University

Rotterdam, Rotterdam, The Netherlands

\section{Synonyms}

Excellence; Experience; Expert performance; Expertnovice research

\section{Definition}

According to Webster's online dictionary, an expert is someone "having, involving, or displaying special skill or knowledge derived from training or experience." The term expertise is often used in a relative sense in educational research. In expert-novice research, an expert is someone who has the knowledge required to perform a certain task, distinguishing him or her from a novice who is not able to perform that task. This task, however, can still be a relatively simple one in the domain. On a domain level, expertise may also refer to the quantity and quality of experience in a domain, with different stages being distinguished on the route from novice to expert. Finally, expertise can be defined in terms of exceptional performance in a domain; expert performance research investigates the consistently superior performance of individuals who excel at representative tasks within that domain.

\section{Theoretical Background}

As should be clear from the definition section, there are different approaches to the study of "expertise" (Ericsson et al. 2006). A lot of research has been conducted on expert-novice differences in performance. This research has shown that experts excel mainly in their domain of expertise, are faster than novices at performing skills, perform their tasks (almost) error free, have superior short-term and long-term memory, and have deeper and more principled problem representations than novices, who tend to build superficial representations of a problem (see e.g., Chi et al. 1988; Ericsson et al. 2006). This research tends to focus mostly on performance of specific tasks within a domain, which do not necessarily have to be very challenging or advanced ones (e.g., it can also be relatively simple math or physics problems). In this sense, 
the use of the term "expert" in expert-novice research is very relative (Chi 2006).

Moving from the task to the domain level, several models exist that describe the different stages in skill acquisition. For example, Alexander's (2003) model of domain learning (MDL) focused on different stages of expertise development in academic domains. She distinguished three stages. The first stage is acclimation, in which learners orient to an unfamiliar domain. They have limited and fragmented knowledge about the domain (although they may already have a lot of knowledge about certain tasks or topics related to that domain). The second stage is competence. Competent learners have acquired a substantial body of knowledge in the domain, which is organized in a coherent and principled manner. The third stage, called proficiency or expertise, is characterized not only by a very extensive knowledge base, but also by the ability to contribute new knowledge to the domain. These stages share some characteristics with another well-known model of skill acquisition by Dreyfus and Dreyfus (1980), which distinguishes the stages novice, competence, proficiency, expertise, and mastery. Note though, that according to their model expertise is the highest stage and mastery is not really a separate stage, but rather occurs as "the expert is capable of experiencing moments of intense absorption in his work, during which his performance transcends even its usual high level" (Dreyfus and Dreyfus 1980, p. 14).

Finally, expert performance research focuses on exceptional performance. Ericsson and Lehmann (1996) noted that research on expert-novice differences and skill acquisition in domains has taken a knowledge-based approach to expertise, which equates expertise with having acquired a lot of knowledge during many years of experience in a domain. However, there is evidence (see Ericsson and Lehmann 1996) that experts by this definition often do not show consistent superior performance on relevant tasks in their domain as compared to less-experienced individuals. Expert performance research is concerned with identifying the mechanisms that have enabled individuals to attain expert performance, that is, "consistently superior performance on a specified set of representative tasks for a domain" (Ericsson and Lehmann 1996, p. 277). Expert performance research has shown that it is not so much the amount of experience (i.e., quantity) in a domain that is relevant for acquiring expert performance, but rather the amount of deliberate efforts made to improve performance (i.e., quantity plus quality). As Ericsson, Krampe, and TeschRömer (1993) argued, expert performance is acquired by extensive engagement in relevant practice activities for improving performance, which they referred to as deliberate practice. Deliberate practice activities are at an appropriate, challenging level of difficulty, and enable successive refinement by allowing for repetition, giving room to make and correct errors, and providing informative feedback to the learner (Ericsson et al. 1993; Ericsson and Lehmann 1996).

\section{Important Scientific Research and Open Questions}

The goal of expert-novice and expert performance research is not just to describe differences in task performance between more and less skilled individuals, but to use this information to help less skilled individuals attain higher or even excellent levels of knowledge and skill (Chi 2006). However, when translating findings from expertise research to educational settings, there are several challenges. First and foremost, applying these principles requires more flexible, individually adaptive instruction than is currently possible for teachers in classroom settings, even though e-learning programs might provide a way to circumvent this problem. Secondly, the main principles of deliberate practice seem relatively easy to implement in educational practice and have also been advocated by educational researchers and educators, such as working on tasks of a challenging level of difficulty (cf. the concept of zone of proximal development). However, while it may be relatively easy to assess whether a student has no knowledge at all about a task or can perform a task well, it is much more difficult to assess what a students' level of expertise is and what a challenging level of difficulty would be for him or her. The techniques used in expertise research to gain insight into acquired knowledge structures (e.g., verbal protocols, eye tracking, recall tasks; see, e.g., Ericsson and Lehman 1996; Ericsson et al. 2006) tend to be rather time consuming and hence unsuitable for rapid use in educational practice, but educational research has started to identify alternatives, especially in e-learning environments (e.g., Kalyuga and Sweller 2005), and this is an avenue of research requiring further exploration. A third issue is that deliberate practice requires people to stretch 
themselves to a higher level of performance, which demands full concentration and is effortful to maintain for extended periods. People do not engage in deliberate practice because it is inherently enjoyable, but because it helps them improve their performance, which they are highly motivated to do. In academic domains, however, this high level of motivation is not a given, and a major open question is how to persuade students to engage in such deliberate practice activities over extended periods of time. Finally, a lot of research on expert performance and deliberate practice has relied on retrospective techniques (e.g., interviews, diary studies) in trying to identify why some people attained excellence and others did not. Prospective experimental or longitudinal research on how deliberate practice leads to excellence in domains seems to be desirable.

\section{Cross-References}

- Adaptive Instruction Systems and Learning

- Cognitive Skill Acquisition

- Complex Skill Acquisition

- Deliberate Practice and Its Role in Expertise

Development

- Development of Expertise

- Feedback and Learning

- Information Processing Theory/Models

- Learning About Complex Domains

- Long-Term Expertise Development

- Long-Term Learning in Soar

- Motivation, Volition, and Performance

- Novice Learning

- Professional Learning and Development

- Skill Acquisition/Learning

Z Zone of Proximal Development

\section{References}

Alexander, P. A. (2003). The development of expertise: The journey from acclimation to proficiency. Educational Researcher, 32, $10-14$.

Chi, M. T. H. (2006). Two approaches to the study of experts' characteristics. In K. A. Ericsson, N. Charness, P. Feltovich, \& R. R. Hoffman (Eds.), The Cambridge handbook of expertise and expert performance (pp. 21-30). Cambridge, UK: Cambridge University Press.

Chi, M. T. H., Glaser, R., \& Farr, M. (Eds.). (1988). The nature of expertise. Hillsdale, NJ: Erlbaum.

Dreyfus, S. E., \& Dreyfus, H. L. (1980). A five-stage model of the mental activities involved in directed skill acquisition. Berkeley, CA: Operations Research Center, University of California.
Ericsson, K. A., \& Lehmann, A. C. (1996). Expert and exceptional performance: Evidence of maximal adaptations to task constraints. Annual Review of Psychology, 47, 273-305.

Ericsson, K. A., Krampe, R. T., \& Tesch-Römer, C. (1993). The role of deliberate practice in the acquisition of expert performance. Psychological Review, 100, 363-406.

Ericsson, K. A., Charness, N., Feltovich, P., \& Hoffman, R. R. (Eds.). (2006). The Cambridge handbook of expertise and expert performance. Cambridge, UK: Cambridge University Press.

Kalyuga, S., \& Sweller, J. (2005). Rapid dynamic assessment of expertise to improve the efficiency of adaptive e-learning. Educational Technology Research and Development, 53, 83-93.

\section{Expertise Development}

Long-Term Learning in Soar

\section{Expertise Reversal Effect}

- Role of Prior Knowledge in Learning Processes

\section{Expert-Novice Research}

Expertise

\section{Explanation Based Generalization}

Deductive Learning

\section{Explanation Based Learning}

Analytic Learning

- Deductive Learning

\section{Explanatory Inference}

Abductive Learning 


\section{Explanatory Style}

Attribution Theory of Motivation

\section{Explanatory Support for Learning}

\author{
Kirsten BERTHOLD \\ Department of Psychology, University of Bielefeld, \\ Bielefeld, Germany
}

\section{Synonyms}

Instructional explanations

\section{Definition}

Explanatory support for learning includes instructional explanations that are aimed at teaching and sharing with others a particular aspect of the learning contents (Leinhardt 2001, 2010). Instructional explanations are a central deliberate act of teaching and are one of the most prevalent strategies in instruction, for example, in classroom teaching, textbooks, human tutoring, peer learning, and computer-based learning environments. They are correct, complete, and coherent. Therefore, they are qualified to (a) efficiently introduce learners to a new learning content to enable a basic understanding, (b) elaborate concepts and procedures to deepen the understanding of the learners further, and (c) clarify difficult aspects of the learning contents when learners get stuck. Despite the very common use of instructional explanations, empirical studies show that very often, they have no positive effects on learning outcomes. This ineffectiveness might be attributed to two central problems: (a) instructors do not adapt their explanations to the knowledge of the learner (problem of lacking adaptation) and (b) learners often show a mental passivity that leads to shallow processing of the explanation (problem of shallow processing).

\section{Theoretical Background}

In the following, the origin of the notion is explained and theoretical claims with respect to the notion are discussed. Afterward, well-documented examples are presented.

\section{Origin of the Notion}

From a philosophical point of view, explanations exist as answers to some sort of actual or implied query (Leinhardt 2001, 2010). According to Leinhardt, the type of explanation, with respect to the queries, is what determines whether an explanation is a common, disciplinary, self, or instructional one. Common explanations occur all of the time in everyday face-to-face conversation and include informal, localized descriptions. Disciplinary explanations answer queries embedded in the discipline itself and use a somewhat formal and even ritualized form of language. Self-explanations are constructed to serve the needs of the self; thus, language can be internal, fragmented, and colloquial as well as fuzzy. In contrast to common, disciplinary, and self-explanations, instructional explanations are designed to explicitly teach - to specifically communicate and share some portion of the subject matter to others, the learners. Compared to disciplinary explanations, the form of instructional explanations is less formal.

\section{A Discussion of Theoretical Claims with Respect to the Notion}

In this section, theoretical claims with respect to (a) the problem of lacking adaptation and (b) the problem of shallow processing of explanations are discussed. These theoretical claims are an important starting point to exploit the potential of instructional explanations.

\section{Problem of Lacking Adaptation}

Instructional explanations should be adapted to the learner's needs in order to maximize learning potential. Webb and Palinscar (1996) emphasized that instructional explanations should be relevant to the learner's particular lack of understanding or misunderstanding. Only then might explanations close gaps in the learner's understanding, remove misconceptions, and foster the integration of new information in the existing knowledge base. To be able to provide adaptive explanations, instructors have to diagnose the learner's prior knowledge and possible misconceptions (Wittwer et al. 2010). However, instructors frequently fail to assess a learner's understanding. The difficulties with assessing a learner's understanding might be due 
to the following reasons (see Wittwer et al.): (a) Instructors might tend to use their own knowledge as a basis for assuming what a learner already knows; (b) Instructors often do not possess strategies for diagnosing a learner's knowledge level; (c) Instructors might experience cognitive overload when the demands of diagnosing within the process of explaining exceeds the limited capacity of their working memory.

\section{Problem of Shallow Processing}

The success of instructional explanations depends not only on the instructors and their adaptation to the learner's prior knowledge but also on the learners. Learners often show a mental passivity that leads to shallow processing of the explanations. As a consequence of shallowly processing instructional explanations, the learners do not actively integrate the new information in their own knowledge base. Against this background, Berthold and Renkl (2010) suggest that providing explanations should be a two-step procedure: the first didactic step includes providing an instructional explanation. This is obvious; however, it is not sufficient. In a second didactic step, instructors should elicit an active processing by "inviting" the learners to further process the instructional explanation. Typically, this second didactic step is neglected even though it is crucial for using the potential of the explanation. With respect to the second step, there is one central principle: the principle of prompting or training focused processing. This principle suggests that learners should focus on the central concepts and principles to be learned (e.g., biological principles such as the principle of segregation in genetics or mathematical principles such as the multiplication rule in probability theory).

\section{Well-Documented Examples}

As explained in the last section, the ineffectiveness of instructional explanations might be attributed to problems of adaptation on the part of the instructor and shallow processing on the part of the learner. In the following examples, instructional support methods that address these problems are introduced.

\section{Instructional Method to Address the Problem of Lacking Adaptation}

Wittwer et al. (2010) proposed that instructional explanations should be adapted to an individual learner's prior knowledge. They developed a computer-based approach called "Assessment Tool" to provide instructors with information about a learner's knowledge with respect to the learning content. For example, instructors receive information on their computer screen about the learner's general experience level in a knowledge domain and information about the learner's specific knowledge with regard to central concepts and principles of the learning domain. In this way, the instructor is able to diagnose the prior knowledge of the learner and, consequently, generate learner-tailored instructional explanations.

\section{Instructional Methods to Address the Problem of Shallow Processing}

Berthold and Renkl (2010) introduce two types of instructional assistance to prevent shallow processing of instructional explanations: $>$ prompts or $\boldsymbol{\text { training }}$ for focused processing regarding the central principles and concepts of the explanation. Prompts are questions or hints embedded in the learning environment that induce productive learning processes. They are designed to overcome superficial processing. Training is conducted prior to the actual learning environment. Berthold and Renkl integrated specific prompts in computer-based learning environments. In a computer-based learning environment including instructional explanations on probability theory, we included prompts regarding the central principles and concepts of the explanation such as "why do you calculate the total possible outcomes by multiplying?" To answer this prompt, the learners had to transfer the instructional explanation on the principle of multiplication for the acceptable outcomes to the multiplication of the possible outcomes. Thus, the general goal of the prompts was to induce focused processing of the central concepts and principles of the provided instructional explanation (e.g., the principle of multiplication). The prompts had to be answered in a written format. An example of a student response is: "because after each of the five possible outcomes, there are again five possible outcomes available."

A training intervention addressing focused processing in the domain of biology consisted of the following main elements: (1) Explication of the learning goals of the training. (2) Presentation of three aspects of theoretical information about focused processing of instructional explanations: (a) The 
training emphasized that it is important to concentrate on the central concepts and principles included in the instructional explanation. (b) On the basis of the - rule-example-rule sequence of Slavin (2007) that includes presenting a rule, giving examples afterward, and then showing how examples illustrate the rule, the training taught the learners to redirect examples presented after the explanation to the central principles in the explanation. Thereby, the learner illustrates why the example exemplifies the principle. (c) Information was given on how to understand why a principle or concept is the way it is. (3) Presentation of corresponding examples from the domain of biology. (4) Demonstration of a cognitive model of how to successfully process explanations. (5) Practice.

\section{Important Scientific Research and Open Questions}

In the following, a brief review of the relevant empirical research with respect to adaptation and focused processing of explanations is provided. Afterward, tobe-expected developments are discussed.

\section{A Brief Review of the Relevant Empirical Research}

\section{Adaptation}

Wittwer et al. (2010) tested their approach "Assessment Tool" that provides instructors with information about a learner's understanding in a series of experiments in different learning situations. The findings suggest two main conclusions. First, information about a learner's prior knowledge proved to be helpful for the instructors to provide learner-tailored explanations. Second, learner-tailored explanations fostered the processing of new information and enhance a deep understanding of the information processed.

\section{Focused Processing of Explanations}

According to studies of Berthold and Renkl (2010) in the domains of probability theory and tax law, prompts that are integrated in a specific learning environment can foster focused processing during working on the learning environment and deepen understanding assessed by a posttest. In addition, they developed a training intervention that addressed focused processing in the domain of biology. This training intervention fostered the quality of focused processing and learning outcomes in a subsequent learning environment on mathematics (between-domain transfer from biology to mathematics). These findings suggest that prompts or training for focused processing regarding the central principles and concepts of the explanation are especially effective with respect to fosteringfocused processing of the explanations and learning outcomes.

\section{To-be-Expected Developments}

In a nutshell, there are three main expected developments with respect to research on instructional explanations. (a) Future studies should investigate whether prompts or training for focused processing of instructional explanations are even more effective if the explanations are adapted to the learners' prior knowledge. Combining adaptive explanations with prompts or training for focused processing might help learners integrate the new knowledge in their own knowledge base to an even greater extent. (b) Currently, the focus of research on instructional explanations is on cognitive factors. However, it is likely that noncognitive factors such as acceptance of the learning environment, motivation, emotion, and social relatedness are relevant with respect to fostering an active processing of explanations, especially in self-regulated learning. It is crucial that cognitive and motivational factors are integrated in research on instructional explanations because both are relevant for successful learning from explanations - not only in the sense of main effects but also in the sense of interactions. (b) The empirical research presented in this contribution was conducted in laboratory experiments. An important next step is to employ diagnostic tools as well as prompts and trainings with respect to focused processing in school as well as other educational settings and evaluate their effects. In this context, interdisciplinary research of the didactics, educational sciences, and educational psychology of explanations could integrate different perspectives on providing and processing instructional explanations.

\section{Cross-References}

- Guided Learning

- Situated Prompts in Authentic Learning Environments 


\section{References}

Berthold, K., \& Renkl, A. (2010). How to foster active processing of explanations in instructional communication. Educational Psychology Review, 22, 25-40. doi:10.1007/s10648-010-9124-9.

Leinhardt, G. (2001). Instructional explanations: A commonplace for teaching and location for contrast. In V. Richardson (Ed.), Handbook of research on teaching (pp. 333-357). Washington, DC: American Educational Research Association.

Leinhardt, G. (2010). Introduction: Explaining instructional explanations. In M. K. Stein \& L. Kucan (Eds.), Instructional explanations in the disciplines (pp. 1-5). New York: Springer.

Slavin, R. E. (2007). Educational psychology: Theory and practice (8th ed.). Boston: Allyn \& Bacon.

Webb, N. M., \& Palinscar, A. S. (1996). Group processes in the classroom. In D. C. Berliner \& R. C. Calfee (Eds.), Handbook of educational psychology (pp. 841-873). New York: Simon \& Schuster Macmillan.

Wittwer, J., Nückles, M., \& Renkl, A. (2010). Using a diagnosis-based approach to individualize instructional explanations in computer-mediated communication. Educational Psychology Review, 22, 9-23.

\section{Explicit}

Prospective and Retrospective Learning in Mild Alzheimer's Disease

\section{Explicit and Implicit Learning}

The definitions of implicit and explicit learning originate in the field of psychology. It generally focuses on the absence or presence of conscious operations as a crucial distinguishing factor. This is in line with Ellis' (1994) definitions of implicit and explicit learning in second language acquisition research. Implicit learning is typically defined as "acquisition of knowledge about the underlying structure of a complex stimulus environment by a process which takes place naturally, simply and without conscious operation," while explicit learning is said to be characterized by "more conscious operation where the individual makes and tests hypotheses in a search for structure."

Explicit learning is equivalent with intentional learning of information. For example, memorizing a list of word pairs would be an example of explicit learning. Implicit learning is typically done in the absence of conscious awareness. Implicit knowledge is typically acquired over many different episodes. For example, learning to ride a bicycle would be an example of implicit learning: there is not usually one particular point in time that one can say, "That is when I learned to ride a bicycle"; rather, the knowledge is gained slowly over time.

\section{References}

Ellis, R. (1994). The study of second language acquisition. Oxford: Oxford University Press.

\section{Explicit and Procedural- Learning-Based Systems of Perceptual Category Learning}

\author{
Shawn Ell ${ }^{1}$, Steven Hutchinson ${ }^{2}$, W. T. Maddox ${ }^{3}$ \\ ${ }^{1}$ Department of Psychology, Maine Graduate School of \\ Biomedical Sciences, University of Maine, Orono, \\ ME, USA \\ ${ }^{2}$ Department of Psychology, University of Maine, \\ Orono, ME, USA \\ ${ }^{3}$ Department of Psychology, University of Texas, \\ Austin, TX, USA
}

\section{Synonyms}

Categorization; Declarative memory; Hypothesistesting system; Implicit learning; Information integration; Nondeclarative memory; Rule based

\section{Definition}

Category learning is the process of establishing a memory trace that improves the efficiency of assigning novel objects to contrasting groups. Memory itself is dependent upon different cognitive and neural systems. Similarly, category learning depends upon multiple learning systems, each dependent upon a different memory system.

The two most extensively studied category learning systems are the explicit and procedural-learning based systems. The explicit system is highly dependent upon - working memory and executive attention, and learns via a logical, hypothesis-testing process. In contrast, the procedural-based system is not dependent 
upon working memory and executive attention. Instead, the procedural-based system incrementally acquires associations between stimuli and the appropriate categorization response via a trial-and-error learning process.

\section{Theoretical Background}

Researchers in such diverse fields as reasoning, discrimination learning, function learning, language acquisition, motor learning, and category learning have argued for the existence of multiple processing systems. Perhaps, the most prolific of these arguments has been made in the memory literature where it is now widely accepted that memory is not unitary, but rather dependent upon multiple cognitive and neural systems. Given the dependence of category learning on memory, it is not surprising that the idea that distinct learning systems contribute to category learning has been suggested by many researchers over the past 30 years.

Multiple systems theorists generally agree that one category learning system is an explicit memory-based system (e.g., Ashby et al. 1998). The explicit system is constrained to use logical rules that are applied to make categorization judgments. At the cognitive level, rule learning depends critically upon the ability to maintain the current rule in working memory, retrieve previous rules from long-term memory, the selection of novel rules, and the ability to switch executive attention among competing rules. The rules used by the explicit system are accessible to conscious awareness and can be described verbally. At the neural level, the explicit system depends primarily upon a network of cortical (prefrontal cortex, anterior cingulate) and subcortical ( $\downarrow$ basal ganglia, thalamus, and midbrain dopaminergic cells) structures.

The nature and characteristics of other category learning systems have been a matter of debate, but considerable evidence supports the existence of a procedural-learning based system (Ashby et al. 1998). The procedural system learns by incrementally associating stimuli with a particular categorization response. In contrast to the explicit system, the procedural system does not have a strong dependence upon the maintenance and manipulation of categorization rules and, as a result is not dependent upon working memory. Instead, the procedural system is critically dependent upon trial-by-trial, corrective feedback to strengthen the appropriate stimulus-response associations. Although learning (i.e., improvement in performance) itself is not implicit, the strategies involved in the formation of the stimulus-response associations are not accessible to conscious awareness. Thus, during learning, it is assumed that individuals are not aware of the decision strategy used by the procedural system. At the neural level, the procedural system depends upon a network of cortical (premotor cortex) and subcortical (basal ganglia, thalamus, and midbrain dopaminergic cells) structures that is distinct from the explicit system.

Critically, the explicit and procedural systems operate in parallel and compete for control of the observable categorization response (Ashby et al. 1998). Initially, there is a bias toward the explicit system, but this bias can shift in favor of the procedural system under the appropriate conditions. For instance, if logical rules are unsuccessful or if explicit system processing is disrupted, the bias will shift in favor of the procedural system.

The explicit and procedural systems differ in their flexibility and ability to generalize. The explicit system is constrained to use logical rules that are easily verbalizable and, as a consequence, is somewhat limited in the range of categorization tasks it can learn. The explicit system, however, is able to successfully generalize learned rules beyond the training stimuli. Computationally, the procedural system uses a nonparametric classifier that is capable of mimicking many different decision strategies. Thus, in contrast to the explicit system, the procedural system is far more flexible during learning. The procedural system, however, is severely limited in its ability to generalize beyond the stimulus-response associations that were learned during training.

The majority of data that has been used to characterize the explicit and procedural systems comes from two types of category learning tasks: rule based and information integration (Ashby and Ell 2001). Rulebased tasks are those in which the categories can be learned by an explicit reasoning process. In many applications, only one stimulus dimension is relevant and a participant's task is to learn the relevant dimension and the placement of the optimal decision rule (Fig. 1a-b). In other cases, all stimulus dimensions are relevant and the participant must learn the optimal decision rule on each dimension (Fig. 1c). Although the Fig. 1 category structures may seem quite different 


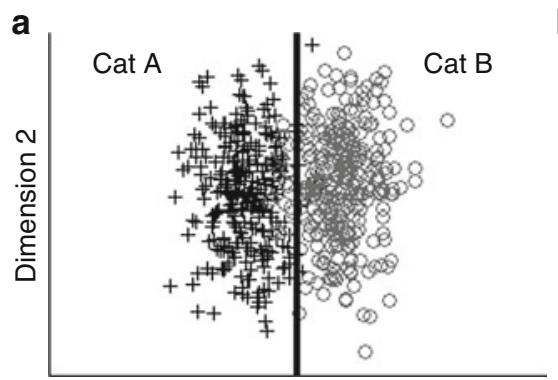

Dimension 1

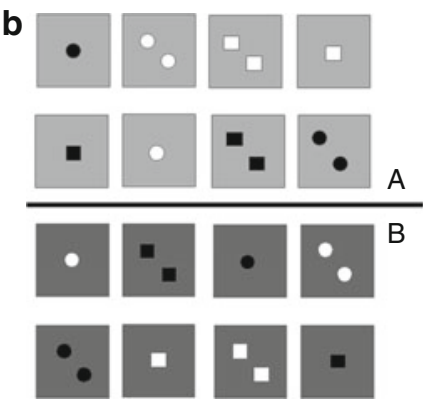

Explicit and Procedural-Learning-Based Systems of Perceptual Category Learning. Fig. 1 Three examples of rulebased category structures. (a) The scatterplot represents stimuli that vary along two, continuously valued dimensions with each point representing an individual stimulus. Although the stimuli vary along both dimensions, only dimension 1 is relevant for categorization. The solid line is the optimal decision rule and can be described as: respond category (cat) A if the stimulus is low on dimension 1; otherwise respond category B. (b) Stimuli that vary along four (background color, symbol color, symbol shape, and symbol number), binary-valued dimensions with only background color being relevant for categorization. The optimal decision rule could be described as: respond category A if the background color is light gray, otherwise respond category B. (c) A rule-based category structure with four categories. In contrast to Fig. 1a, both dimensions are relevant for categorization. The solid line is the optimal decision rule and can be described as: respond category $A$ if the stimulus is low on dimension 1 and low on dimension 2; respond category B if the stimulus is low on dimension 1 and high on dimension 2; respond category $C$ if the stimulus is high on dimension 1 and high on dimension 2; otherwise respond category $\mathrm{D}$

on the surface, optimal performance can be achieved by the use of logical rules in all cases.

In contrast, information-integration tasks are those in which information from multiple dimensions is integrated prior to making a categorization response (Fig. 2a-c). The type of integration required could take any number of forms, from a weighted combination of the two dimensions to more holistic processing to the incremental acquisition of stimulus-response associations. Unlike rule-based tasks, participants have difficulty verbalizing the optimal decision strategy in information-integration tasks, despite being able to successfully learn the categories. All of the informationintegration category structures in Fig. 2 were designed with the constraint that the use of simple (e.g., one- or two-dimensional) logical rules cannot result in optimal performance.

Rule-based and information-integration tasks are thought to be probes for the explicit and procedural systems, respectively. For instance, logical rules would be successful on rule-based tasks, thus the system bias should remain in favor of the explicit system (recall that there is an initial bias toward the explicit system). In contrast, on information-integration tasks, logical rules would be only marginally successful. Thus, the system bias is predicted to shift in favor of the procedural-based system. Nevertheless, it is important to note that the use of a particular task does not guarantee the use of a particular system. Instead, it is the type of decision strategy that is a more reliable proxy for a particular system (e.g., a strategy assuming the use of a logical rule versus a strategy assuming that a holistic representation of the stimulus is associated with a categorization response). Thus, one must go beyond traditional accuracy-based analyses in order to investigate the type of decision strategy that was used, as qualitatively different strategies can predict almost identical accuracy rates.

The distinction between the explicit and procedural systems has been highly successful in generating a priori predictions for performance on rule-based and information-integration tasks (Maddox and Ashby 2004). For instance, learning in the explicit system is dependent upon the ability to maintain rules in working memory, switch executive attention away from unsuccessful rules, and switch executive attention to alternative rules. Such processing requirements successfully predict that manipulations that increase 


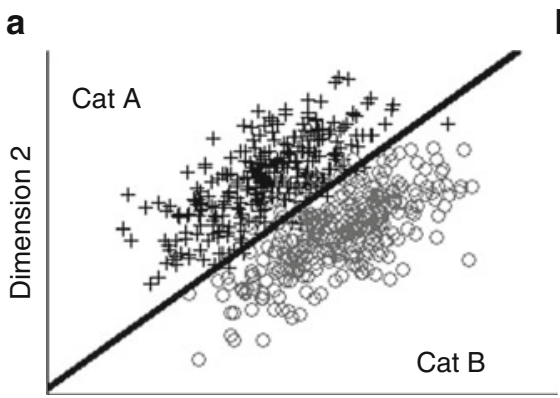

Dimension 1

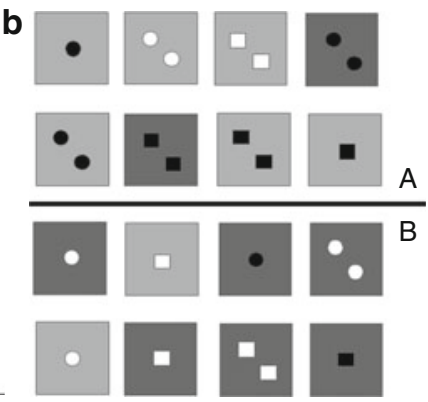

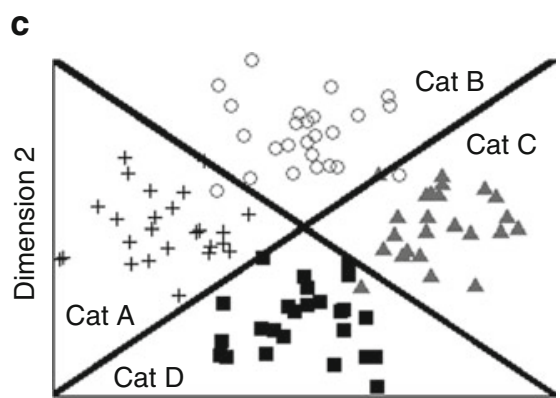

Dimension 1

Explicit and Procedural-Learning-Based Systems of Perceptual Category Learning. Fig. 2 Three examples of information-integration category structures. (a) The scatterplot represents stimuli that vary along two, continuously valued dimensions with each point representing an individual stimulus. In order to learn these categories, individuals must integrate the information from the two stimulus dimensions. The optimal decision boundary (the solid line) is included for presentation purposes and is not intended to be a representation of what individuals are learning. (b) An informationintegration category structure constructed from four, binary-valued dimensions. In this example, shape is irrelevant. For the three relevant dimensions, one level is arbitrarily assigned a numerical value of +1 : symbol color of black, background color of light gray, and numerosity of two. The other levels are assigned a numerical value of 0: symbol color of white, background color of dark gray, and numerosity of one. If the sum of the values on the relevant dimensions is greater than 1.5 , the stimulus is assigned to category A; if less than 1.5 , the stimulus is assigned to category B. (c) An informationintegration category structure with four categories

demands upon working memory and executive attention should impair accuracy on rule-based tasks to a greater extent than on information-integration tasks. At the neural level, rule-maintenance and manipulation in the explicit system are predicted to be highly dependent upon a prefrontal-basal ganglia network and dopaminergic functioning. Consistent with this prediction, dysfunction in prefrontal-basal ganglia networks resulting either from dopamine depletion in individuals with Parkinson's disease or basal ganglia stroke impairs performance on rule-based tasks.

In contrast, learning in the procedural system is dependent upon associating relatively high-level stimulus and response representations. Such learning requires a consistent stimulus-response mapping. Thus, as predicted, disrupting the consistency of this mapping impairs performance on informationintegration tasks to a greater extent than on rulebased tasks. At the neural level, a network linking high-level visual regions (e.g., inferotemporal cortex), the basal ganglia, and high-level motor regions (e.g., premotor cortex) is argued to mediate the learning of stimulus-response associations. A critical feature that distinguishes this from other types of associative learning mechanisms (e.g., Hebbian learning) is that learning in the procedural system is dependent upon a dopamine-mediated reward signal in the basal ganglia that occurs shortly after response selection. More specifically, a phasic burst of dopamine in response to unexpected, correct feedback strengthens stimulus-response associations (via long-term potentiation), whereas no change or a phasic decrease in dopamine weakens stimulus-response associations (via long-term depression). This neurally inspired model correctly predicts, for example, that performance on information-integration tasks should be severely impaired when feedback is absent or simply delayed.

\section{Important Scientific Research and Open Questions}

Great strides have been made in recent years in understanding the computational requirements of the explicit and procedural systems. We also have considerable information about the neural substrates of these systems. This knowledge, however, is rather coarse (e.g., we know that the prefrontal cortex is a critical component of the explicit system) as we know relatively 
little about the interaction of computational requirements and neural structures on a real-time basis. Although invaluable, many popular techniques in cognitive neuroscience have limited temporal resolution (e.g., behavioral experiments with neurologically impaired populations, neuroimaging) or are limited in their ability to measure activity in key regions such as the basal ganglia (e.g., electroencephalography). Addressing this issue will depend in part on the use of less widespread techniques such as magnetoencephalography and the continued development of detailed computational cognitive neuroscience models. Moreover, recent $\boldsymbol{~ o p t o g e n e t i c ~ t e c h n i q u e s ~ i n ~ a n i m a l ~ m o d e l s ~}$ that provide the ability to measure the impact of selective modification of neural pathways in real time will likely prove important for understanding the microcircuitry of the explicit and procedural systems.

Perhaps, one of the greatest challenges to further characterization of the explicit and procedural systems, and to multiple systems theories in general, is to understand how the systems interact (Ashby and Maddox 2011). Although limited, the available data suggest that the explicit and procedural systems operate in parallel and compete with each other, but there is little data to rule out other types of interactions (e.g., cooperation). Even if competition is the correct assumption, there is very little data to guide theorizing on how competition is resolved on a trial-by-trial basis. We know even less about how these systems interact at the neural level. Such knowledge will be critical for a complete understanding of the interaction of the explicit and procedural systems as well as interaction with other putative category learning systems.

Recent research suggests that the contribution of the explicit and procedural systems declines as categorization automaticity develops (Ashby et al. 2007). Neurally, this involves a transfer of control from subcortical to cortical networks. Of course, on a day-today basis we are constantly challenged by information that is inconsistent with automatized behaviors. Thus, it would seem necessary that we be able to switch from networks mediating automatic categorization to the explicit and procedural systems in order to update existing category representations. Such switching abilities should be incorporated into future neurocomputational models of categorization.

It is becoming increasingly clear that motivational factors influence the functioning of the explicit and procedural systems (Maddox and Markman 2010). For instance, the extent to which global motivational incentives match the local task incentives can have a profound impact on functioning within the explicit system by facilitating the use of appropriate rule-based strategies and encouraging the discovery of less salient rules. In addition, the social stressors we encounter in our daily lives can have a profound impact on the explicit system with enhancement or impairment depending on individual differences in the stress response. Due to the competitive nature of the interaction between the explicit and procedural systems, a social stressor that impairs the explicit system can actually enhance the procedural system. There is no mechanism within current models of category learning to adequately explain these data.

\section{Cross-References}

- Basal Ganglia Learning

- Categorical Learning

- Categorical Representation

- Classification Learning

- Dual Process Models of Information Processing

- Explicit Versus Implicit Learning

- Mathematical Models/Theories of Learning

\section{References}

Ashby, F. G., \& Ell, S. W. (2001). The neurobiology of human category learning. Trends in Cognitive Science, 5, 204-210.

Ashby, F. G., \& Maddox, W. T. (2011). Human category learning 2.0. Annals of the New York Academy of Sciences, 1224, 147-161.

Ashby, F. G., Alfonso-Reese, L. A., Turken, A. U., \& Waldron, E. M. (1998). A neuropsychological theory of multiple systems in category learning. Psychological Review, 105, 442-481.

Ashby, F. G., Ennis, J. M., \& Spiering, B. J. (2007). A neurobiological theory of automaticity in perceptual categorization. Psychological Review, 114, 632-656.

Maddox, W. T., \& Ashby, F. G. (2004). Dissociating explicit and procedural-learning based systems of perceptual category learning. Behavioral Processes, 66, 309-332.

Maddox, W. T., \& Markman, A. B. (2010). The motivation-cognition interface in learning and decision-making. Current Directions in Psychological Science, 19, 106-110.

\section{Explicit Instruction}

- Historical Thinking 


\section{Explicit Learning}

Meaningful Learning in Economic Games

\section{Exploitation}

This concept has been defined by March (1991) in opposition to exploration. The exploitation of existing options relates to the rationalisation of production systems, organizational processes, and collective competences. As March himself coins it, exploitation includes such things as refinement, choice, production, efficiency, selection, implementation, and execution.

\section{References}

March, J. G. (1991). Exploration and exploitation in organizational learning. Organization Science, 2(1), 71-87.

\section{Exploration}

This concept has been defined by March (1991) in order to investigate organisations aimed at developing new solutions. Exploration supposes demonstrating flexibility, risk taking, experimentation, discovery, and an ability to variation. It supposes actions, behaviors and organisational processes fostering the discovery of brand new solutions to newly identified problems.

\section{References}

March, J. G. (1991). Exploration and exploitation in organizational learning. Organization Science, 2(1), 71-87.

\section{Exposure-Based Perceptual Learning}

\author{
Hoon Choi, Takeo Watanabe \\ Boston University, Boston, MA, USA
}

\section{Definition}

The term "exposure-based perceptual learning (EPL)" refers to long-lasting improvements in performance of perceptual tasks as a result of passive exposure to a feature of stimuli. Whereas a traditional view of “ perceptual learning” emphasizes a subject's active performance during training, EPL shows that perceptual learning can occur even when subjects are passively exposed to the feature.

The concept of EPL originated from one seminal study finding "task-irrelevant perceptual learning (TIPL)" (Watanabe et al. 2001). The study showed that, contrary to the traditional view that perceptual learning can only occur with focused attention to the features relevant to a given task, perceptual learning occurred on a subliminal feature that was irrelevant to the main task (such that the learned feature could not be attended to during training). TIPL implied that perceptual learning was accomplished not only by active performance but also by passive exposure to a feature.

In a more recent study (Seitz et al. 2009), this passive perceptual learning was also found even when subjects performed a task of passively viewing stimuli without any action during training. Because the exposed feature in their experiment can hardly be defined as a task-irrelevant feature, the term EPL was employed instead of TIPL. Thus, EPL is a broader concept than TIPL. However, both EPL and TIPL are employed with the same meaning, and TIPL is the more popular term.

\section{Theoretical Background}

What to learn and what not to learn? This is a critical question for those interested in adaptation of the human brain to its environment. The surrounding environment continuously changes. Some of the changes are very critical and long lasting, while other changes are trivial and temporary. Therefore, the brain needs to selectively accept some changes and reject others. This "stability-plasticity dilemma" (Grossberg 1980; Spanis and Squire 1987) is also applied to perceptual learning.

A traditional view suggested that attention played a critical role in determining what to learn and what not to learn in perceptual learning, based on numerous psychophysical and physiological studies. Ahissar and Hochstein (1993) showed that practicing a task of discriminating the orientation of a "global" shape did not evoke a learning effect in the task of detecting 
a "local" element, even though both tasks employed exactly same stimuli. One physiological study found the orientation tuning curves of macaque monkey V1 cells to be sharpened in relation to the location of the stimulus with which they performed an orientation discrimination task during the training session, but not in relation to the location of the stimulus that was merely exposed (Schoups et al. 2001).

This hypothesis was challenged by recent findings of EPL (or TIPL) in which learning of a feature occurred in the absence of attention to the feature (Watanabe et al. 2001). In their experiment, subjects were asked to select and identify letters from a rapid serial visual presentation (RSVP) display, a rapid succession of letters and numbers, which was presented against a background of dynamic random-dot (DRD) display consisting of coherently moving dots (signal) and randomly moving dots (noise). Because this motion display was not related to the central task, the display was out of the focus of the subject's attention. This task-irrelevant motion display, moreover, had a very weak motion direction signal. The display was on a $5 \%$ coherence level $-5 \%$ of the motion display consisted of coherently moving dots and 95\% consisted of randomly moving dots. In this low coherent motion display, the direction of coherently moving dots could hardly be perceived, detected, or recognized; in other words, the strength of the motion signals was below the threshold, or subliminal. Despite its irrelevancy and indifferentiability, repeated exposure to the background motion display improved subjects' sensitivity specifically to the trained direction. This finding implied that focused attention to a feature was not necessary for perceptual learning of the feature. Rather, perceptual learning could occur only with simple exposure to the feature.

\section{Important Scientific Research and Open Questions}

The discovery of EPL was striking and interesting, yet at the same time, it complicated the question of selectivity. If perceptual learning of a feature can occur as a result of mere exposure to the feature, how does the brain decide what to learn and what not to? Does EPL mean that all stimuli we experience in our daily life are learned? Recent studies have suggested the selectiveness of EPL, showing the following four conditions in which EPL has occurred.

\section{The Processing of a Task-Target}

EPL occurred only when a feature was presented while subjects were engaged in the processing of a task target. In a study of Seitz and Watanabe (2003), subjects were asked to select and identify letters from RSVP (consisting of letters and numbers) on a background of DRD(which was a task-irrelevant stimulus). Whereas EPL of DRD was found when DRD was presented with a target letter, EPL was not found when DRD was paired with distractor numbers. However, this target-paring benefit was not found when subjects failed to identify a target, even though DRD was presented with a target.

\section{Absence of Attention}

EPL was stronger when a feature of a task-irrelevant stimulus was not attended to. In a recent study (Choi et al. 2009), for task-irrelevant stimuli, two DRDs were presented simultaneously, one on either side of the screen. Using arrows, a subject's attention was oriented to one of the DRDs (because arrows could trigger attentional orienting such that a subject's attention would automatically be directed to the place where the arrowhead pointed). Based on numerous previous studies showing focused attention to facilitate perceptual learning of task-relevant feature, stronger EPL was expected to occur on attended DRD rather than unattended DRD. Contrary to this expected result, EPL occurred only on DRD on the unattended side and not on DRD on the attended side.

\section{Subliminality of Task-Irrelevant Stimuli}

EPL occurred when a task-irrelevant stimulus was subliminal rather than suprathreshold. A recent study explored a potential relationship between EPL and magnitude of a task-irrelevant stimulus (Tsushima et al. 2008). In their experiment, for task-irrelevant stimuli, DRD with various coherence levels were employed. It might be predicted, intuitively, that DRD with a higher coherence level would evoke 
a greater learning effect since it would be assumed that a stimulus with a stronger signal should induce a stronger learning signal. However, the results contradicted such predictions. EPL took place for the relatively low coherent motion DRD, but did not for the relatively high coherent motion DRD (50\%), which was obviously suprathreshold.

\section{Provision of Rewards}

EPL was stronger when relevant rewards were provided. In a recent study (Seitz et al. 2009), subjects passively viewed two differently oriented sinusoidal gratings. Whenever one grating with a specific orientation was presented, a small amount of water was delivered through a tube placed in their mouths, while no water was offered when the grating with the other orientation was presented. Because the subjects had been deprived of food and water for $5 \mathrm{~h}$ before the experiment, it was assumed that the water would work as an external reward. The results showed the close relation between an external reward and EPL. Namely, sensitivity to the orientation paired with water drops was increased relative to the sensitivity to the orientation that was not paired with any external reward. Based on this reinforcing effect of rewards, some researchers proposed that a relationship between EPL and target processing (aforementioned) should be understood in terms of rewards. Seitz and Watanabe (2005) have suggested, for example, that successful performance of a given task, such as detecting a target, could generate an internal reward that works like an external reward.

Although EPL provides a new view of perceptual learning, there are several issues that are still controversial. One of them is that EPL is not consistent with several previous studies showing no perceptual learning of unattended features. Several studies of EPL suggested that the inconsistency might be because previous studies employed suprathreshold stimuli as unattended stimuli.

\section{Cross-References}

- Effects of physical context change and perceptual learning on generalization

- Insight in perceptual learning

- Perceptual Learning

- Task-Irrelevant Perceptual Learning

\section{References}

Ahissar, M., \& Hochstein, S. (1993). Attentional control of early perceptual learning. The Proceedings of the National Academy of Science USA, 90, 5718-5722.

Choi, H., Seitz, A. R., \& Watanabe, T. (2009). When attention interrupts learning: Inhibitory effects of attention on TIPL. Vision Research, 49, 2586-2590.

Grossberg, S. (1980). How does a brain build a cognitive code? Psychological Review, 87, 1-51.

Schoups, A., Vogels, R., Qian, N., \& Orban, G. (2001). Practising orientation identification improves orientation coding in $\mathrm{V} 1$ neurons. Nature, 412, 549-553.

Seitz, A., \& Watanabe, T. (2003). Psychophysics: Is subliminal learning really passive? Nature, 422, 36.

Seitz, A., \& Watanabe, T. (2005). A unified model for perceptual learning. Trends in Cognitive Sciences, 9(7), 329-334.

Seitz, A. R., Kim, D., \& Watanabe, T. (2009). Rewards evoke learning of unconsciously processed visual stimuli in adult humans. Neuron, 61(5), 700-707.

Spanis, C. W., \& Squire, L. R. (1987). Stability of long temporal gradients of retrograde amnesia in mice. Behavioral and Neural Biology, 48, 237-245.

Tsushima, Y., Seitz, A., \& Watanabe, T. (2008). Task-irrelevant learning occurs only when the irrelevant feature is weak. Current Biology, 18(12), R516-517.

Watanabe, T., Náñez, J. E., \& Sasaki, Y. (2001). Perceptual learning without perception. Nature, 413, 844-848.

\section{Expression of Emotions}

- Facial Expressions While Performing Problems

\section{Externalizing and Internalizing Behavior}

- Achievement Deficits of Students with Emotional and Behavioral Disabilities

\section{Extinction}

A reduction in conditioned responding that results from omitting the unconditioned stimulus after repeated presentation of the conditioned stimulus (in classical conditioning) or repeated occurrences of the learned response (in instrumental conditioning). 


\section{Extinction Learning}

Catherine A. Hartley ${ }^{1}$, Elizabeth A. Phelps ${ }^{1,2}$

${ }^{1}$ Department of Psychology, New York University,

New York, NY, USA

${ }^{2}$ Department of Neural Science, New York University,

New York, NY, USA

\section{Synonyms}

Inhibitory learning

\section{Definition}

Extinction learning refers to the gradual decrease in response to a conditioned stimulus that occurs when the stimulus is presented without reinforcement. The term "extinction" was first used by Ivan Pavlov in reference to his observation that the conditioned response to a cue that predicted food delivery decreased and eventually disappeared when food no longer followed the cue. In Pavlovian fear conditioning, a previously neutral stimulus comes to elicit a fear response through pairing with an aversive reinforcer, such as an electric shock or a loud noise. During extinction, a new association with the stimulus is learned that inhibits the expression of the original fear memory. Extinction learning serves as the foundation of exposure therapy, which is commonly used to treat pathological fear.

\section{Theoretical Background}

Russian scientist Ivan Pavlov first documented the phenomenon of extinction in his seminal classical conditioning experiments (Pavlov 1927). In these studies, dogs were repeatedly given food following the presentation of a tone. After several such pairings, the dogs began to salivate at the presentation of the tone. Pavlov referred to the tone as the conditioned stimulus (CS) and the food as the unconditioned stimulus (US). The innate salivation response to food was termed the unconditioned response (UR), while salivation in response to the tone was referred to as the conditioned response (CR). Once the conditioned response had been learned, repeated presentations of the tone without subsequent food delivery led to a gradual decrease in salivation that Pavlov referred to as the "extinction" of the conditioned response.
While early theories hypothesized that the conditioned response is unlearned during extinction, experimental evidence indicates that the original association between the CS and the US remains intact. Following extinction, several manipulations can bring about the reemergence of the CR (see Bouton 2004, for a review). Conditioned responding typically returns after the mere passage of time, an occurrence known as "spontaneous recovery." Exposure to the US following extinction causes "reinstatement" of the CR. Presentation of the CS outside of the context in which extinction learning takes place leads to "renewal" of the CR. In light of these behavioral phenomena, current theories propose that extinction learning involves the formation of a new inhibitory association with the CS that competes with the original excitatory association for expression.

The brain mechanisms supporting extinction learning have primarily been investigated in studies examining the extinction of conditioned fear (see Quirk and Mueller 2008, for a review), as the neural processes underlying fear learning are relatively well understood. During fear conditioning, neurons in the lateral nucleus of the amygdala encode the association between the CS and the US, which controls the expression of the CR via projections to the central nucleus of the amygdala. The initial acquisition of extinction learning involves molecular changes within the basolateral amygdala. During the several hours following acquisition, the extinction memory undergoes consolidation, during which plasticity in the basolateral amygdala, ventromedial prefrontal cortex, and the hippocampus serve to stabilize the memory. Following consolidation, retrieval of the extinction memory elicits increased activity in the ventromedial prefrontal cortex, which projects to the intercalated cell masses of the amygdala that in turn inhibit central nucleus output. Projections from the hippocampus to the vmPFC and the basolateral amygdala modulate fear expression based on whether the context predicts threat or safety.

The persistence of fear in the absence of any imminent threat is a hallmark of anxiety disorders. Impaired ability to acquire or retrieve extinction learning may underlie such pathological anxiety. Exposure therapy, in which individuals are exposed to stimuli associated with previous trauma in a safe context, attempts to treat anxiety disorders by fostering fear extinction. In addition, research in animals demonstrating the 
enhancement of extinction through the administration of various pharmacological agents suggests a promising avenue for improving current therapies (see Anderson and Insel 2006, for a review). Recent research in animal models and humans suggests that when extinction learning occurs shortly after the retrieval of a fear memory, this new learning may disrupt the $\checkmark$ reconsolidation of the original fear memory leading to a persistent reduction in fear.

\section{Important Scientific Research and Open Questions}

At present, little is known about the factors that give rise to differences among individuals in extinction learning and retention. Recent exploratory research suggests that variation in brain structure, genetic polymorphisms that influence molecular learning processes, and experimental factors ranging from maternal care to chronic stress all contribute to variation in extinction. Further research into the origins of such individual differences may elucidate what renders a person vulnerable to or resilient against anxiety disorders.

The extinction of appetitive associations has not been widely studied to date. Preliminary research suggests that the vmPFC and the amygdala also play a role in appetitive extinction (see Quirk and Mueller 2008, for a review); however, the involvement of additional regions involved in reward processing (e.g., striatum) remains to be clarified. Reward-related classical and instrumental conditioning processes are thought to support the development and maintenance of drug addiction. A better understanding of the mechanisms underlying the extinction of appetitive associations may yield novel approaches to treating substance abuse disorders.

\section{Cross-References}

- Classical Conditioning, Anticipatory Learning

- Fear Conditioning in Animals and Humans

- Internal Reinforcement Hypothesis

- Memory Consolidation and Reconsolidation

- Pavlovian Conditioning

\section{References}

Anderson, K. C., \& Insel, T. R. (2006). The promise of extinction research for the prevention and treatment of anxiety disorders. Biological Psychiatry, 60, 319-321.
Bouton, M. E. (2004). Context and behavioral processes in extinction. Learning \& Memory, 11, 485-494.

Pavlov, I. P. (1927). Conditioned reflexes: An investigation of the physiological activity of the cerebral cortex (trans: Anrep, G. V.). London: Oxford University Press.

Quirk, G. J., \& Mueller, D. (2008). Neural mechanisms of extinction learning and retrieval. Neuropsychopharmacology, 33, 56-72.

\section{Extraversion}

Extraversion is a personality dimension characterized by sociability, enthusiasm, risk taking, and needing the company of others. This dimension has been described in several models of personality including Eysenck's personality theory and the Big Five factor model.

\section{Extraversion, Social Interaction, and Affect Repair}

\author{
Adam A. Augustine ${ }^{1}$, Scott H. Hemenover ${ }^{2}$
}

${ }^{1}$ Department of Psychology, Washington University in St. Louis, St. Louis, MO, USA

${ }^{2}$ Western Illinois University, Macomb, IL, USA

\section{Synonyms}

Affect regulation; Personality; Self-regulation; Socialization

\section{Definition}

Extraversion is one of the Big Five personality traits (extraversion, neuroticism, agreeableness, conscientiousness, and openness to experience) used to encompass human personality. Extraversion is comprised of affective, social, and responsivity components with extraverts (vs. introverts) experiencing more positive affect, engaging in social interaction to a greater degree, and showing higher levels of reward responsivity. Affect repair is a form of affect regulation in which individuals attempt to decrease levels of negative (i.e., sadness, anxiety) and increase or maintain levels of positive (i.e., happiness, enthusiasm) affect. 


\section{Theoretical Background}

Research on links between personality and socialization processes has consistently found the personality trait of extraversion to be a key predictor of a wide variety of social behaviors. Extraverts (vs. introverts) report spending more time with other people, possess more social resources, and report a more pleasant affective profile when in the presence of others. Those higher (v. lower) in extraversion also experience higher levels of positive affect in general, react more strongly to positive stimuli, and experience a slower rate of decay of positive affect (Hemenover 2003). Based on the extant literature, social attention has been suggested as the core of extraversion (Ashton et al. 2002). Thus, the extraverted individual's favorable affective profile may be due to an increased enjoyment of, and engagement in, socialization; however, empirical and theoretical work on affect regulation suggests additional possible mechanisms. Some strategies for affect regulation are more effective than others (Augustine and Hemenover 2009) and extraverts tend to use those effective strategies to a greater degree (Larsen and Augustine 2008). Considering the relationships among extraversion, socialization, and affect regulation, one might suppose that if an extravert (vs. introvert) uses social regulatory strategies then they would experience an additive benefit to their positive affect. In contrast to this intuitive prediction, recent research reveals that extraverts may not gain greater affect repair benefits from socialization than do introverts.

\section{Important Scientific Research and Open Questions}

The research to date indicates that all individuals, not just extraverts, may gain affective benefits from engaging in social interaction. The first set of studies to examine this (Fleeson et al. 2002) is based on the fact that individuals regularly display various levels of the Big-five personality trait. For instance, the degree to which one acts extraverted varies greatly over time, at times being consistent with low (e.g., solitary activities), and other times with high, extraversion. Thus, the highly extraverted individual does not always display high levels of extraverted behavior, and the introvert sometimes displays relatively high levels of extraverted behavior. Fleeson and colleagues (2002) found that, on a momentary basis, one's current (or state) level of extraversion predicted positive affect in the same manner as does typical (or trait) extraversion. To further explore this, Fleeson and colleagues (2002) had participants either act introverted or act extraverted during a social interaction. They found that those who acted extraverted experienced more positive affect as a result of the social interaction, regardless of their trait standing on extraversion. These findings suggest that acting extraverted provides the same momentary boost to positive affect as being extraverted provides to typical positive affect levels.

In a second examination of the possibility that all individuals, regardless of their personality, can benefit from socialization, Augustine and Hemenover (2008) examined the impact of socialization and extraversion on affect repair ability. In this study, participants underwent a procedure designed to make them experience increased negative affect (i.e., a negative affect induction). The participants then described/discussed a benign topic (grocery lists and film formats) either alone or in the presence of a confederate that had been trained to respond in a positive and supportive manner. Results for the participants who described the topic alone were consistent with the increased repair abilities of the extravert; extraverts experienced greater affect repair in this solitary condition. However, when in the presence of the positive interaction partner, these differences disappeared; all individuals, regardless of extraversion, experienced relatively equivalent affect repair in this social condition. Thus, consistent with the findings of Fleeson and colleagues (2002), this study (Augustine and Hemenover 2008) found that all individuals can affectively benefit from socialization.

These studies suggest that introverts may be able to alleviate their relatively lower levels of positive affect through an increased participation in social interaction. With this relative equity of affective benefits from socialization occurring across the range of extraversion, then why do introverted individuals not seek out social situations to a greater degree? Although there are a number of possible explanations, one possibility is that introverts simply have not learned that they gain affective benefits from social interaction. Introverts engage in relatively little socialization and do not desire increased social engagement. In addition, introverts often report that socialization can be cognitively and physically taxing, despite the described research showing that there are affective benefits for socialization. 
Thus, with relatively little desire to socialize and a (potentially false) assumption regarding negative effects of socialization, the introverted (vs. extraverted) individual may set up a self-fulfilling prophecy, whereby they go into social situations expecting poor outcomes and achieve poor outcomes as a result of their expectations. In addition, the introverted individual may encode or recall the results of a socialization attempt in line with their expectations (i.e., negative outcomes), rather than the actual positive affect benefits that result from socialization.

Another possibility is that introverts do not gain these affective benefits in a wide range of social situations. Both experimental studies (Augustine and Hemenover 2008; Fleeson et al. 2002) relied on a social interaction task that was very positive. However, the introverted individual does not possess the superior socialization abilities of the extraverted individual. Thus, without an overtly positive interaction, the socialization ability deficits of the introvert may not be sufficient to elicit a positive interaction in cases where the social interaction is neutral or negative. Although, if an introvert were to engage in more interactions, they have the potential to learn the skills necessary for positive socialization and, perhaps, would achieve the positive affective benefits of social interaction across a wider variety of social situations.

\section{Cross-References}

- Emotion Regulation

- Introversion - Extraversion

- Neuroticism as a Predictor of Mood Change

- Self-regulation and Motivation Strategies

- Socio-emotional Aspects of Learning

\section{References}

Ashton, M. C., Lee, K., \& Paunonen, S. V. (2002). What is the central feature of extraversion? Social attention versus reward sensitivity. Journal of Personality and Social Psychology, 83, 245-252.

Augustine, A. A., \& Hemenover, S. H. (2008). Extraversion and the consequences of social interaction on affect repair. Personality and Individual Differences, 44, 1151-1161.

Augustine, A. A., \& Hemenover, S. H. (2009). On the relative effectiveness of affect regulation strategies: A meta-analysis. Cognition \& Emotion, 23, 1181-1220.

Fleeson, W., Malanos, A. B., \& Achille, N. M. (2002). An intraindividual process approach to the relationship between extraversion and positive affect: Is acting extraverted as "good" as being extraverted? Journal of Personality and Social Psychology, $83,1409-1422$.
Hemenover, S. H. (2003). Individual differences in rate of affect change: Studies in affective chronometry. Journal of Personality and Social Psychology, 85, 121-131.

Larsen, R. J., \& Augustine, A. A. (2008). Basic personality dispositions: Extraversion/neuroticism, BAS/BIS, positive/negative affectivity, and approach/avoidance. In A. J. Elliot (Ed.), Handbook of approach and avoidance motivation (pp. 151-164). Hillsdale: Lawrence Erlbaum Associate.

\section{Extrinsic Goals}

- Goal Theory/Goal Setting

\section{Eyeblink Conditioning}

\section{E. James Kehoe}

School of Psychology, University of New South Wales, Sydney, NSW, Australia

\section{Synonyms}

Eyelid conditioning

\section{Definition}

Eyeblink conditioning is a type of classical conditioning that has been used for 90 years to investigate associative learning and, increasingly, its neural substrates. The most common conditioned stimulus (CS) has been a pure tone, although other auditory, visual, and, occasionally, tactile stimuli have been used. The unconditioned stimulus (US) has usually been a mild puff of air to the cornea or a brief electrical pulse delivered to the skin around the eye. Both USs reliably elicit a brief closure of the outer eyelids and, where present, the inner eyelid - the nictitating membrane - as the unconditioned response (UR). Depending on a variety of conditions, CS-US pairings produce the acquisition of a conditioned response (CR) to the CS (Kehoe and Macrae 2002).

\section{Theoretical Background}

Experimentation using eyeblink conditioning has made fundamental and continuing contributions to theories of conditioning at behavioral and neural levels. 
The basic learning process is now usually described by an error-correction rule, the best known of which is the Rescorla-Wagner Rule. In addition, eyeblink conditioning has been used to test many other models of conditioning, for example, the Pearce-Hall model and Wagner's SOP model (Wagner and Brandon 1989). These abstract models have progressively been merged with neural models of eyeblink conditioning. These neural models are based primarily on the architecture of the cerebellar cortex, the deep cerebellar nuclei, and brainstem pathways that are the primary physical substrate of eyeblink conditioning in many species, including humans (Christian and Thompson 2003).

\section{Important Scientific Research and Open Questions}

Basic CR acquisition is characterized by a progressive increase in initiation of eyelid closure during the interstimulus interval (ISI) between CS onset and US onset. However, the CR does not duplicate the UR. The UR is initiated within $40 \mathrm{~ms}$ and reaches its peak closure within another $40 \mathrm{~ms}$. In contrast, the time course of the CR is determined by the ISI. As a rule of thumb, CR initiation occurs at least half way through the ISI, and then the recruitment of the CR is graded such that the peak closure is aligned with the time that US had been delivered. Figure 1 shows the time course of eyeblink CRs as recorded on CS-alone trials in groups of rabbits trained with ISIs of $125 \mathrm{~ms}, 250 \mathrm{~ms}$, and $500 \mathrm{~ms}$. As a rule of thumb, ISIs around 250500 ms reliably yield the most rapid CR acquisition.

Eyeblink conditioning has been studied in species as varied as sharks, mice, rats, guinea pigs, rabbits, ferrets, cats, and humans. From the 1920s to 1960s, the bulk of studies were conducted using humans. During the 1970s-1990s, studies using the rabbit became prominent for both behavioral and neurophysiological purposes. More recently, rats and mice have been increasingly favored for neurophysiological studies. The study of human eyeblink conditioning has also experienced a modest resurgence.

The effects of a large number of manipulations have been systematically delineated. Their effects have generally been consistent across species. The manipulations can be roughly grouped into four classes:

Control comparisons entail the presentations of the CS-alone, US-alone, and explicitly unpaired

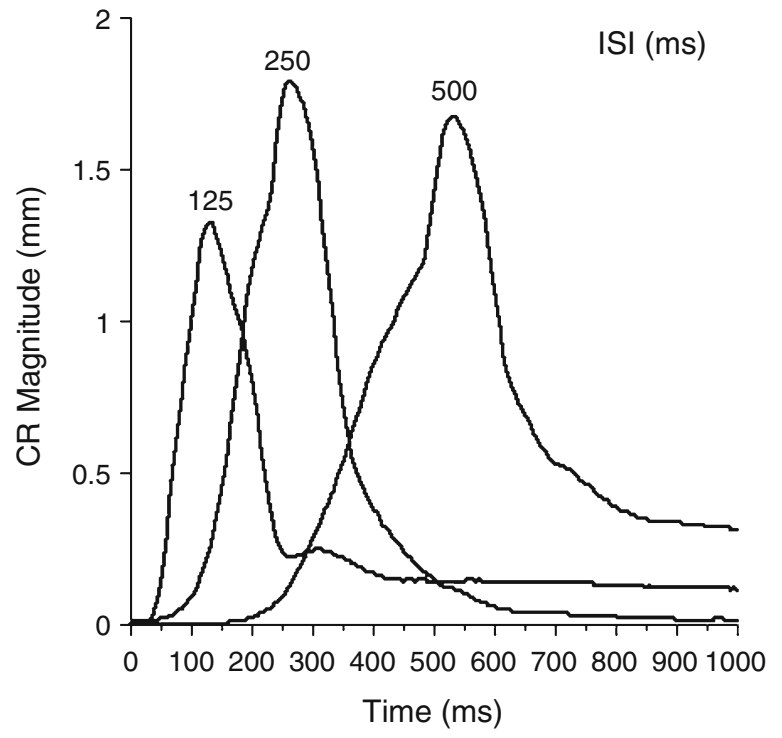

Eyeblink Conditioning. Fig. 1 The averaged time course of CRs in the rabbit nictitating membrane using a $50 \mathrm{~ms}$ tone CS in trace conditioning using ISIs of 125,250 , and $500 \mathrm{~ms}$. The figure is based on unpublished data from the author's laboratory

presentations of the CS and US to separate groups. Responses during these presentations provide an estimate of contamination from background blink rate, sensitization, and other nonassociative processes to the observed likelihood of CRs from CS-US pairings. Nonassociative responding is negligible in the rabbit, but it can rise to $25 \%$ of total responding in some species.

Stimulus parameters include CS intensity, CS duration, US intensity, and US duration. All other things being equal, the rate of $\mathrm{CR}$ acquisition is a positive function of all these variables. In recent years, trace conditioning, in which the CS duration is shorter than the CS-US interval, has been of interest, because it appears to be more susceptible to deleterious effects of hippocampal lesions, in comparison to delay conditioning, in which the CS duration fills the CS-US interval.

Training schedules include manipulations of the sequence and spacing of CS-US pairings, CS-alone trials, and, less commonly, US-alone trials. For example, a partial reinforcement schedule is one in which CS-US pairings are intermixed with CS-alone trials. 
CR acquisition on CS-US pairings tolerates the introduction of CS-alone trials and only begins to decline when the proportion of CS-alone trials exceeds $25 \%$ of the total. When a large number of CS-alone trials precede CS-US pairings, CR acquisition can be retarded. This effect is known as latent inhibition. Conversely, when a large number of CS-alone trials follow CS-US pairings, the frequency of CRs shows a progressive decline known as extinction. Extinction of the observable CR does not mean that the underlying CS-US association has been erased. For example, after the $\mathrm{CR}$ has been eradicated by repeated sessions of extinction training, rapid reacquisition of the CR occurs when CS-US pairings are reintroduced.

Multiple CS manipulations use two or more CSs, for example, a tone and a light. To describe such manipulations, the CSs are often designated with letters $(\mathrm{A}, \mathrm{B}, \ldots)$, presentation of the US is designated with a plus sign (+), and the absence of the US is designated with a minus sign (-). For example, differential conditioning entails the pairing of one CS with the US $(\mathrm{A}+)$ and the presentation of the other CS without US $(B-)$. The two CSs can be presented simultaneously in a compound paired with the US $(\mathrm{AB}+)$ or presented sequentially prior to the US $(\mathrm{A} \rightarrow \mathrm{B}+)$. Combined with manipulations of the stimulus parameters and training schedule, the variety of manipulations conducted to date has been large and growing. Among others, they include overshadowing, in which the intensity of one element in $\mathrm{AB}+$ training is manipulated; blocking, in which one element is given paired training $(\mathrm{A}+)$ before $\mathrm{AB}+$ training; conditioned inhibition, in which paired trials for one element $(\mathrm{B}+)$ are intermixed with non-reinforced $\mathrm{AB}$ - trials; and serial feature positive discrimination, in which sequential compound trials $(\mathrm{A} \rightarrow \mathrm{B}+)$ are intermixed with nonreinforced trials for the second element $(\mathrm{B}-)$.

Experimentation with eyeblink conditioning continues to mirror contemporary issues in the wider research concerning learning at behavioral and neural levels. At a neural level, the role of cerebral structures like the hippocampus and prefrontal cortex are being progressively delineated. At both levels, the time course of the CR and its dependence on the ISI has been of the objects of sophisticated real-time models. In these models, error-correction rules have been combined with assumptions that the CS generates a sequence of microstimuli that allow different portions of the CS gain a greater or smaller share of the total associative strength depending on their proximity to the US.

The eyeblink CR has often been construed as depending on instrumental effects, that is, eyelid closure is thought to be reinforced by its potential ability to attenuate the aversive impact of the US. For a US that has a physical impact on the eye, such as an airpuff, this hypothesis has considerable face validity. However, where the US only has an impact near the eye, like the electrotactile US, the face validity of an instrumental hypothesis becomes increasingly speculative. Empirically, when explicit avoidance contingencies for eyelid closure have been implemented, the results have been opposite to an instrumental hypothesis. Specifically, response-contingent reductions in US intensity, including complete avoidance, have reduced the rate and asymptote of CR acquisition. Conversely, response-contingent increases in US intensity - "punishment" - have tended to increase the rate of CR acquisition (Coleman 1975).

The resurgence of human eyeblink conditioning has reinvigorated long-standing debates whether it is governed by two distinct processes, one being a basic associative process like that studied in nonhuman animals and a uniquely human process. This latter process has been variously described in terms of volition, cognition, contingency awareness, and/or declarative memory. The basic appeal of these latter conceptions arises from the ability of humans to close their eyelids in response to a verbal command and their ability to provide ratings, verbally or on dial, that a CS precedes a US.

\section{Cross-References}

- Associative Learning

- Computational Models of Classical Conditioning

- Conditioned Inhibition

- Conditioning

- Connectionist Theories of Learning

- Discrimination vs. Generalization

- Extinction Learning

- Latent Inhibition

- Mechanisms of Learning

- Pavlovian Conditioning

- Temporal Learning in Humans and Other Animals 


\section{References}

Christian, K. M., \& Thompson, R. F. (2003). Neural substrates of eyeblink conditioning: Acquisition and retention. Learning \& Memory, 11, 427-455.

Coleman, S. R. (1975). Consequences of response-contingent change in unconditioned stimulus intensity upon the rabbit (Oryctolagus cuniculus) nictitating membrane response. Journal of Comparative and Physiological Psychology, 88, 591-595.

Kehoe, E. J., \& Macrae, M. (2002). Fundamental behavioral methods and findings in classical conditioning. In J. W. Moore (Ed.), A neuroscientist's guide to classical conditioning (pp. 171-231). New York: Springer.
Wagner, A. R., \& Brandon, S. E. (1989). Evolution of a structured connectionist model of Pavlovian conditioning (AESOP). In S. B. Klein \& R. R. Mowrer (Eds.), Contemporary learning theories: Pavlovian conditioning and the status of traditional learning theory (pp. 149-190). Hillsdale: Erlbaum.

\section{Eyelid Conditioning}

- Eyeblink Conditioning 